University of Tennessee Health Science Center

UTHSC Digital Commons

\title{
Biochemical and Immunological Mechanisms Underlying Differential Interaction of Superantigens with Host Immunogenetic Factors in Streptococcal Sepsis
}

Mohammed Nooh

University of Tennessee Health Science Center

Follow this and additional works at: https://dc.uthsc.edu/dissertations

Part of the Bacteria Commons, and the Bacterial Infections and Mycoses Commons

\section{Recommended Citation}

Nooh, Mohammed, "Biochemical and Immunological Mechanisms Underlying Differential Interaction of Superantigens with Host Immunogenetic Factors in Streptococcal Sepsis" (2008). Theses and Dissertations (ETD). Paper 196. http://dx.doi.org/10.21007/etd.cghs.2008.0227. 


\title{
Biochemical and Immunological Mechanisms Underlying Differential Interaction of Superantigens with Host Immunogenetic Factors in Streptococcal Sepsis
}

\author{
Abstract \\ Group A streptococci (GAS) are serious human pathogens that can cause a wide array of diseases \\ ranging from pharyngitis to streptococcal toxic shock (STSS), which is caused by excessive cytokines \\ responses triggered by streptococcal superantigens (Strep SAgs). SAgs interact simultaneously with HLA \\ class II molecules on antigen presenting cells (APC) and with the T cell receptor beta chain variable \\ elements (TCR V) and cause excessive stimulation of both cell types and massive release of \\ inflammatory mediators.
}

The Strep SAgs include the streptococcal pyrogenic exotoxins (Spes), which play a crucial role in the pathogenesis of severe invasive streptococcal diseases, including Strep toxic shock syndrome (STSS). However, not all septic individuals infected with SAg producing GAS develop STSS. Our epidemiologic studies showed that individual HLA class II allelic variation play a crucial role in modulating the severity of invasive GAS diseases. Certain HLA class II alleles and haplotypes confer strong protection from STSS (DRB1*1501/DQB1*0602) or increase the risk for it (DRB1*1401/DQB1*0503 and DRB1*0701/ $\mathrm{DQB1}$ *0202), whereas others behave in a neutral fashion with respect to disease severity. The main aim of this thesis was to validate the direct role of HLA class II variation in modulating the severity of GAS sepsis and investigate molecular mechanisms underlying the differential effect of HLA class II variation on SAgs responses in vitro and in vivo.

We validated our epidemiological findings and confirmed the direct role of HLA class II allelic variation in modulating the severity of GAS sepsis using HLA transgenic ( $\mathrm{tg}$ ) mice expressing alleles of interest. Splenocytes from mice expressing the protective HLA-DQB1*06 (DQ6) allele mounted significantly lower proliferative and cytokine responses to Strep SAgs, than splenocytes from mice expressing the neutral DRB $1 * 0402 / D Q B 1 * 0302$ (DR4/DQ8) alleles. Furthermore, cross presentation of Strep SAgs to pure T cells by antigen presenting cells (APC) from these HLA-tg mice showed that presentation by HLA-DQ6 APC elicited significantly lower proliferative and inflammatory cytokine responses than by the HLA-DR4/DQ8 APC. These in vitro data were supported by in vivo findings, as the DQ6 mice showed significant resistance to GAS sepsis with lower inflammatory cytokine responses and longer survival, than the DR4/ DQ8 mice.

Similarly, presentation of the Strep SAgs by human APC expressing STSS protective haplotypes elicited significantly lower proliferative and cytokine responses than by APC expressing neutral or high risk haplotypes, even when the responding APC-depleted T cells came from different individuals with different TCR V repertoires. Moreover, we screened SAgs responses over a panel of B lymphoblastiod cell lines (BLCL), expressing the most prevalent HLA class II haplotypes in populations with high incidence of STSS, using pure $T$ cells from different individuals, and reproducibly observed significantly higher responses in the presence of the high-risk haplotypes or neutral than protective haplotypes. These data demonstrate a dominant role of individual HLA class II and not TCR VB repertoire variation in determining the potency or type of responses to the Strep SAgs.

An important finding from the above studies is that the presentation of the Strep SAgs by the protective HLA class II haplotype (DR15/DQ6) elicits high levels of the regulatory cytokine IL-10, and thus may be actively suppressing inflammatory cytokine production. To further investigate this point and to determine if in the clinical setting, humans heterozygous for high risk and protective haplotypes would be protected or prone to STSS, we generated a panel of bare lymphocyte syndrome cells (BLS) (class II negative cells) expressing individual HLA-DR, DQ to delineate their relative contribution to the potency and type of Strep SAg responses. In addition to the mixture of M1T1 SAgs, we also generated a panel of recombinant SAgs 
found in this mixture to investigate the interaction and relative contribution of variable HLA and SAg molecules to the overall response.

To ensure equimolar expression of HLA class II alleles on BLS cells, we utilized the novel technology of 'self cleaving' 2A peptide to generate multicistronic retroviral vectors. The constructs were cloned into pHSPG retroviral vector and packaged into HSPG viral particles that were used to transduce (BLS). DR or $D Q$ and chains were expressed as a single message linked by $2 A$ peptide resulting in coordinated expression of both chains that was confirmed by quantitative flow cytometric analysis of the transduced BLS cells.

Presentation of individual Strep SAgs by BLS cells expressing either of the protective alleles (DR15 or DQ6) induced significantly lower proliferative and cytokine responses than that by BLS cells expressing single alleles on the high-risk haplotypes. Reproducibly, higher levels of the immunosuppressive cytokine $\mathrm{IL}-10$ were produced in the presence of the protective alleles. Thus, confirming our suggestion that protective alleles mediate their effects through active suppression of inflammatory cytokines production.

In an attempt to understand the molecular basis, we investigated if differences in binding affinities between specific SAgs and HLA class II alleles affect response level. In general, there was a correlation between binding affinity of Alexa fluor 647-labeled SAgs to individual class II alleles and response levels, except for SmeZ1 binding to and presentation by DQ alleles, where an inverse correlation was observed. However, Ca flux studies demonstrated good correlation with the observed responses. These data indicate that individual class II alleles differentially bind and present Strep SAgs resulting in significantly different proliferative and cytokines responses. The inverse relation between SmeZ1 responses and its binding affinities to DQ alleles suggest that different combinations of SAgs-HLA alleles may trigger distinct signaling in T cells resulting in variable levels of response.

In conclusion, our study demonstrate dominant and direct role of HLA class II in controlling Strep SAgs responses and resulting disease severity. The data indicate that each SAg-class allele is unique with distinct behavior that cannot be extrapolated to others. Future studies will investigate this point further and determine the interactions between high risk and protective HLA class II alleles, when expressed on the same APC.

\section{Document Type}

Dissertation

\section{Degree Name}

Doctor of Philosophy (PhD)

\section{Program}

Molecular Sciences

Research Advisor

Malak Y. Kotb, Ph.D.

\section{Keywords}

Group A streptococci, Superantigens, HLA class II polymorphism, TCR Vb repertoire variation, Streptococcal toxic shock syndrome, transgenic mice

\section{Subject Categories}

Bacteria | Bacterial Infections and Mycoses | Diseases | Medicine and Health Sciences | Organisms 


\title{
Biochemical and Immunological Mechanisms Underlying Differential Interaction of Superantigens with Host Immunogenetic Factors in Streptococcal Sepsis
}

\author{
A Dissertation \\ Presented for \\ The Graduate Studies Council \\ The University of Tennessee \\ Health Science Center
}

\author{
In Partial Fulfillment \\ Of the Requirements for the Degree \\ Doctor of Philosophy \\ From The University of Tennessee
}

By

Mohammed Mostafa Nooh

May 2008 
Chapter 2 ( 2007 by the American Association of Immunologists, Inc. Chapter 5 (C) 2007 by the American Society for Biochemistry and Molecular Biology. All other materials (C) 2008 by Mohammed M. Nooh.

All rights reserved. 


\section{DEDICATION}

To my loving and encouraging parents

To my incessantly supportive wife

And to my dear and sweet children 


\section{ACKNOWLEDGEMENTS}

I would like to thank my mentor, Dr. Malak Kotb, for offering me the opportunity to pursue my Ph.D. study. I especially thank her not only for her extensive scientific knowledge and teachings, but also for her helpful advice and guidance in many other areas during my graduate studies. Her wonderful patience, trust, support and positive attitude exemplified role model for mentor. I also thank my committee members Dr. AeKyung Yi, Dr. Dario Vignali, Dr. David Brand, and Dr. Marko Radic for their valuable suggestions and positive critique of my research. I additionally thank Dr. Brand for invaluable technical assistance with cell sorting and calcium mobilization studies. Dr. Brand was always genuinely open and willing to offer his time and advice whatever the matter. I would also like to thank Dr. Vignali for assistance with generation of multicistronic 2A-linked constructs. I thank all previous and current members of the Kotb lab, not to mention H Leighton Legros, Ramy Aziz and Rita Kansal, for the family spirit and support I enjoyed throughout my work with them. In addition, I thank Karen Whittington who always lent me a hand when I faced problems using the flow cytometer.

I deeply thank my parents for always encouraging me throughout my life to do my best and for expressing their optimism and confidence in my ability to succeed. Their prayers always paved the way for me and made it possible for me to attain this goal. I cannot find words to express my appreciation for my amazing wife who, besides her duties as a graduate student, bore the main responsibility of caring for our children throughout our time in Memphis; her never ending support and understanding helped me accomplish a project of that magnitude. I thank my friends Walid Awad, Yasser Abd- 
Elrahman, Hossam Ashour, Shehab Ismail, Hossam Abd-Elsamed, Ali Ellbidy for their support during my stay in Memphis; they are the brothers I never had. 


\begin{abstract}
Group A streptococci (GAS) are serious human pathogens that can cause a wide array of diseases ranging from pharyngitis to streptococcal toxic shock (STSS), which is caused by excessive cytokines responses triggered by streptococcal superantigens (Strep SAgs). SAgs interact simultaneously with HLA class II molecules on antigen presenting cells (APC) and with the T cell receptor beta chain variable elements (TCR Vß) and cause excessive stimulation of both cell types and massive release of inflammatory mediators.

The Strep SAgs include the streptococcal pyrogenic exotoxins (Spes), which play a crucial role in the pathogenesis of severe invasive streptococcal diseases, including Strep toxic shock syndrome (STSS). However, not all septic individuals infected with SAgproducing GAS develop STSS. Our epidemiologic studies showed that individual HLA class II allelic variation plays a crucial role in modulating the severity of invasive GAS diseases. Certain HLA class II alleles and haplotypes confer strong protection from STSS (DRB1*1501/DQB1*0602) or increase the risk for it (DRB1*1401/DQB1*0503 and DRB1*0701/DQB1*0202), whereas others behave in a neutral fashion with respect to disease severity. The main aim of this thesis was to validate the direct role of HLA class II variation in modulating the severity of GAS sepsis and investigate molecular mechanisms underlying the differential effect of HLA class II variation on SAgs responses in vitro and in vivo.
\end{abstract}

We validated our epidemiological findings and confirmed the direct role of HLA class II allelic variation in modulating the severity of GAS sepsis using HLA transgenic (tg) mice expressing alleles of interest. Splenocytes from mice expressing the protective 
HLA-DQB1*06 (DQ6) allele mounted significantly lower proliferative and cytokine responses to Strep SAgs, than splenocytes from mice expressing the neutral DRB1*0402/DQB1*0302 (DR4/DQ8) alleles. Furthermore, cross presentation of Strep SAgs to pure T cells by antigen presenting cells (APC) from these HLA-tg mice showed that presentation by HLA-DQ6 APC elicited significantly lower proliferative and inflammatory cytokine responses than by the HLA-DR4/DQ8 APC. These in vitro data were supported by in vivo findings, as the DQ6 mice showed significant resistance to GAS sepsis with lower inflammatory cytokine responses and longer survival, than the DR4/DQ8 mice.

Similarly, presentation of the Strep SAgs by human APC expressing STSS protective haplotypes elicited significantly lower proliferative and cytokine responses than by APC expressing neutral or high risk haplotypes, even when the responding APCdepleted T cells came from different individuals with different TCR V $\beta$ repertoires. Moreover, we screened SAgs responses over a panel of B lymphoblastiod cell lines (BLCL), expressing the most prevalent HLA class II haplotypes in populations with high incidence of STSS, using pure T cells from different individuals, and reproducibly observed significantly higher responses in the presence of the high-risk haplotypes or neutral than protective haplotypes. These data demonstrate a dominant role of individual HLA class II and not TCR Vß repertoire variation in determining the potency or type of responses to the Strep SAgs.

An important finding from the above studies is that the presentation of the Strep SAgs by the protective HLA class II haplotype (DR15/DQ6) elicits high levels of the regulatory cytokine IL-10, and thus may be actively suppressing inflammatory cytokine 
production. To further investigate this point and to determine if in the clinical setting, humans heterozygous for high risk and protective haplotypes would be protected or prone to STSS, we generated a panel of bare lymphocyte syndrome cells (BLS) (class II negative cells) expressing individual HLA-DR, DQ to delineate their relative contribution to the potency and type of Strep SAg responses. In addition to the mixture of M1T1 SAgs, we also generated a panel of recombinant SAgs found in this mixture to investigate the interaction and relative contribution of variable HLA and SAg molecules to the overall response.

To ensure equimolar expression of HLA class II alleles on BLS cells, we utilized the novel technology of 'self cleaving' $2 \mathrm{~A}$ peptide to generate multicistronic retroviral vectors. The constructs were cloned into $\mathrm{pHSPG}$ retroviral vector and packaged into HSPG viral particles that were used to transduce (BLS). DR or DQ $\alpha$ and $\beta$ chains were expressed as a single message linked by $2 \mathrm{~A}$ peptide resulting in coordinated expression of both chains that was confirmed by quantitative flow cytometric analysis of the transduced BLS cells.

Presentation of individual Strep SAgs by BLS cells expressing either of the protective alleles (DR15 or DQ6) induced significantly lower proliferative and cytokine responses than that by BLS cells expressing single alleles on the high-risk haplotypes. Reproducibly, higher levels of the immunosuppressive cytokine IL-10 were produced in the presence of the protective alleles. Thus, confirming our suggestion that protective alleles mediate their effects through active suppression of inflammatory cytokines production. 
In an attempt to understand the molecular basis, we investigated if differences in binding affinities between specific SAgs and HLA class II alleles affect response level. In general, there was a correlation between binding affinity of Alexa fluor 647-labeled SAgs to individual class II alleles and response levels, except for SmeZ1 binding to and presentation by DQ alleles, where an inverse correlation was observed. However, Ca flux studies demonstrated good correlation with the observed responses. These data indicate that individual class II alleles differentially bind and present Strep SAgs resulting in significantly different proliferative and cytokines responses. The inverse relation between SmeZ1 responses and its binding affinities to DQ alleles suggest that different combinations of SAgs-HLA alleles may trigger distinct signaling in $\mathrm{T}$ cells resulting in variable levels of response.

In conclusion, our study demonstrates a dominant and direct role of HLA class II in controlling Strep SAgs responses and resulting disease severity. The data indicate that each SAg-class II allele is unique with distinct behavior that cannot be extrapolated to others. Future studies will investigate this point further and determine the interactions between high risk and protective HLA class II alleles, when expressed on the same APC. 


\section{TABLE OF CONTENTS}

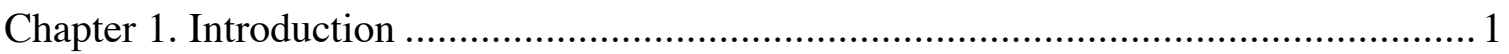

Group A Streptococci and Human Diseases......................................................... 1

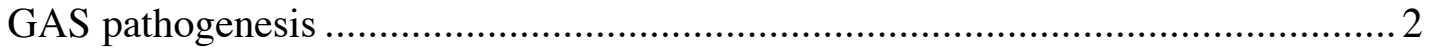

Re-emergence of severe invasive GAS infections .............................................. 2

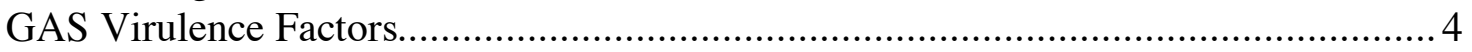

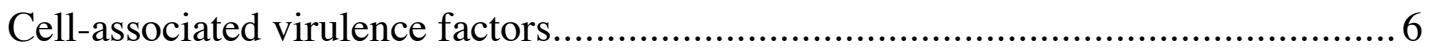

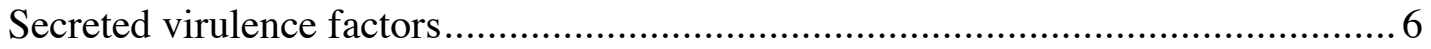

Superantigens' Structure, Function and Interaction with Immune Receptors ............... 11

Conventional antigens (Ag) versus superantigens (SAg) ....................................... 12

Discovery of streptococcal superantigens ........................................................... 13

Superantigens produced by other bacteria......................................................... 15

Structural features of SAgs ............................................................................. 15

Interactions of SAgs with HLA class II molecules .............................................. 20

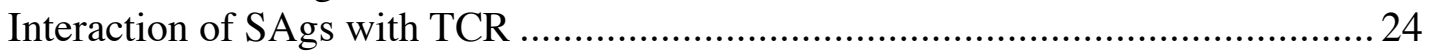

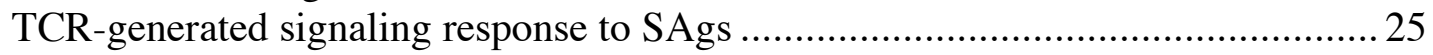

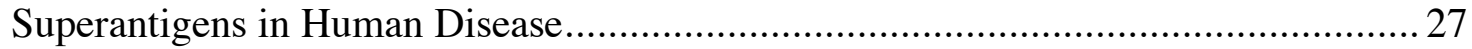

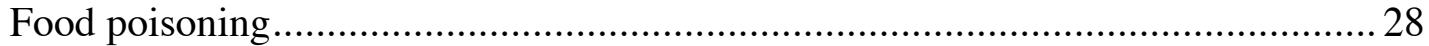

Staphylococcal toxic shock syndrome (staphTSS) …………........................... 28

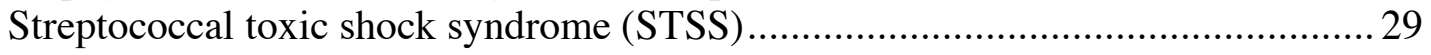

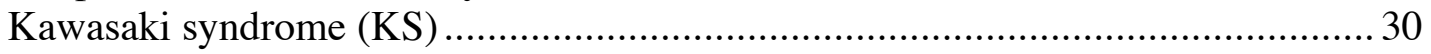

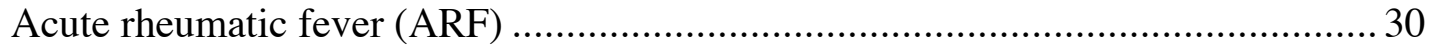

Role of Host Genetics in Infectious Diseases ........................................................... 31

Host genetic involvement in the pathogenesis of infectious diseases ........................31

The same GAS strain is associated with variable infection severity......................... 33

Host risk factors for invasive streptococcal infections............................................... 34

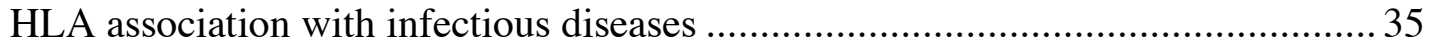

HLA class II polymorphism and SAg responses .................................................... 37

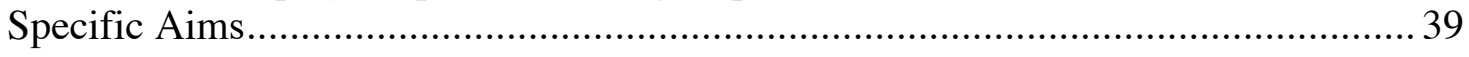

Chapter 2. HLA Transgenic Mice Provide Evidence for a Direct and Dominant

Role of HLA Class II Variation in Modulating the Severity of Streptococcal Sepsis...... 42

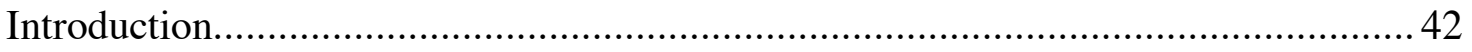

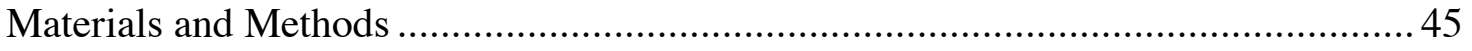

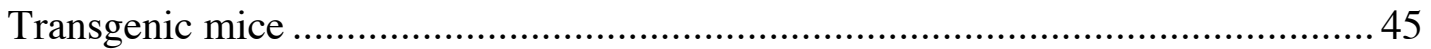

Preparation of partially purified streptococcal SAgs from GAS culture

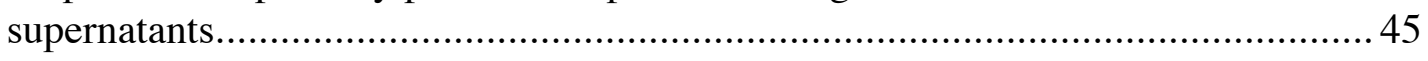

Generation of recombinant GAS superantigens..................................................... 46

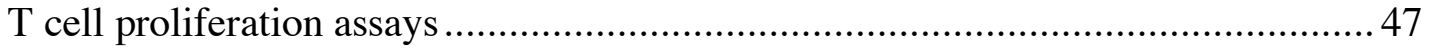

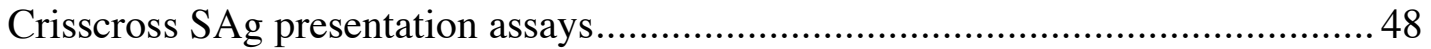

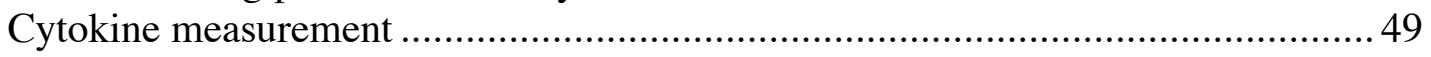


Analysis of the T cell receptor V $\beta$ (TCR V $\beta$ ) repertoire by flow cytometry ...........50

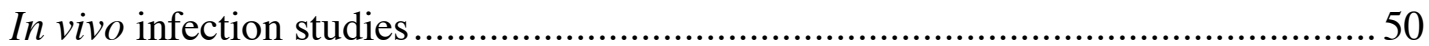

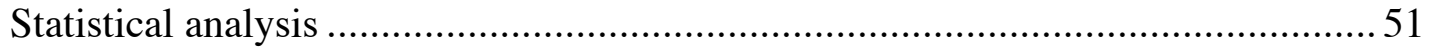

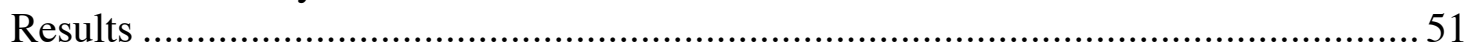

Differential in vitro response of HLA-tg mice splenocytes to M1T1 SAgs............ 51

Dominance of the effect of HLA allelic variation in potentiating M1T1 SAgs

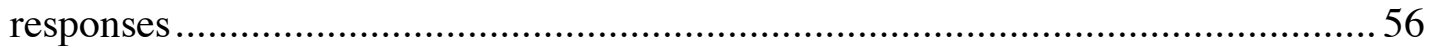

HLA class II allelic variation directly modulates the severity of systemic disease and mouse survival following infection with M1T1 GAS ........................59

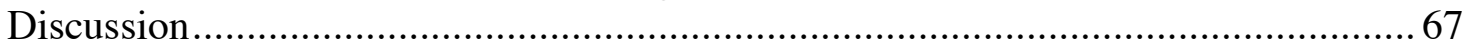

Chapter 3. HLA Allelic Variants Dictate the Overall Cytokine Profile Elicited by Superantigens: Streptococcal Toxic Shock Protective Alleles Elicit IL-10 Production and Diminish Inflammatory Responses .......................................... 71

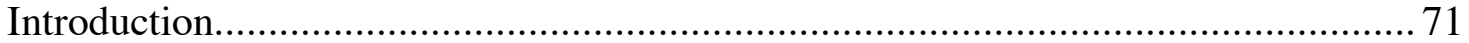

Materials and Methods ..................................................................................... 73

Preparation of partially purified Strep SAgs from GAS culture supernatants......... 73

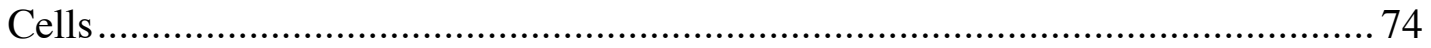

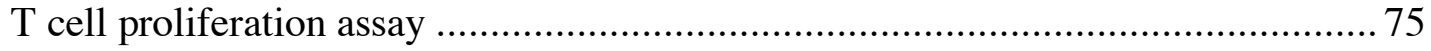

Cytokine assays ........................................................................................... 75

Analysis of the T cell receptor V $\beta$ (TCR V $\beta$ ) repertoire by flow cytometry ........... 76

Flow cytometric assessment of HLA DQ and DR expression level ...................... 76

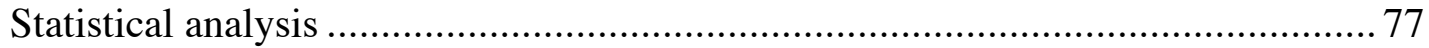

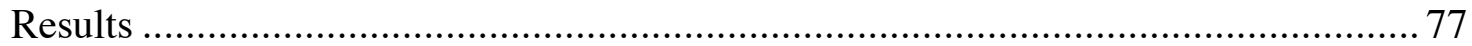

HLA Class II allelic variation plays a dominant role in determining the magnitude and type of Strep SAg responses ................................................ 77

DR15/DQ6 alleles diminish SAg responses by inducing high levels of IL-10 ....... 85

Exogenous IL-10 diminishes SAgs responses induced by high-risk alleles............ 85

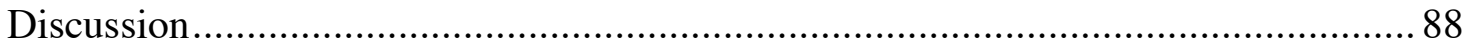

Chapter 4. Molecular Basis for HLA Class II Differential Presentation of Streptococcal Superantigens Involved in GAS Sepsis............................................ 95

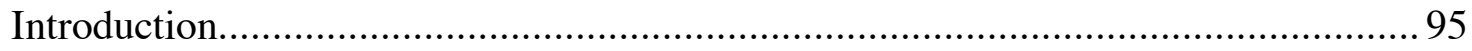

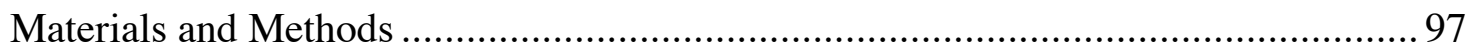

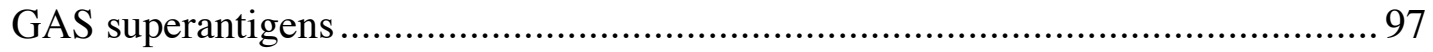

Generation of HLA class II multicistronic vectors ......................................... 98

Virus preparation, transduction and cell sorting ............................................ 98

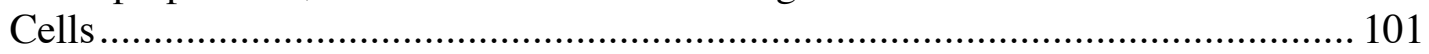

Assessment of HLA DQ and DR expression level .......................................... 104

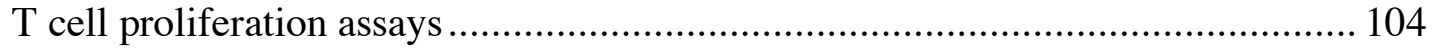

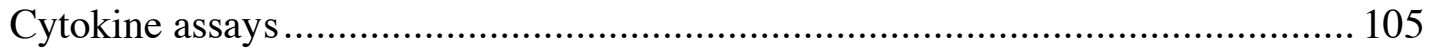

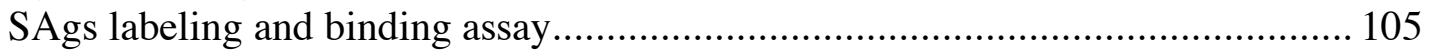

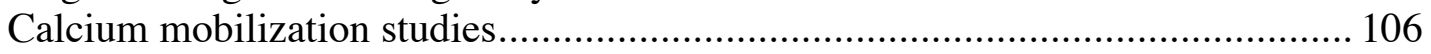

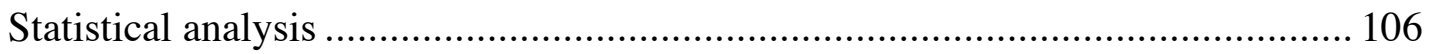




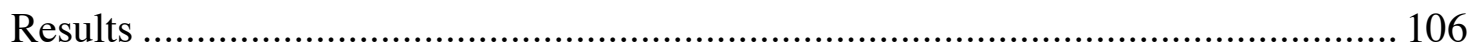

Relative effects of prevalent HLA class II haplotypes on M1T1 SAgs responses . 106

Preferential presentation of individual M1T1 rSAgs by HLA DR or DQ alleles... 110

Binding affinity of rSpeG, rSpeJ and rSmeZ1 to different HLA class II alleles .... 113

Changes in $\left[\mathrm{Ca}^{2+}\right]_{\mathrm{i}}$ levels in response to stimulation with $\mathrm{rSmeZ1}$....................... 118

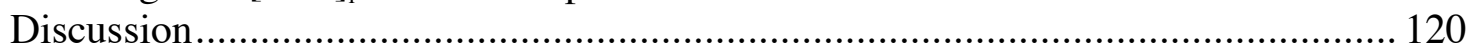

Chapter 5. Streptococcal Mitogenic Exotoxin Z, SmeZ, Is the M1T1 Streptococcal Superantigen Most Susceptible to Degradation by the Streptococcal Cysteine Protease, SpeB ……................................................................................... 124

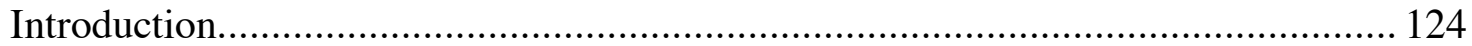

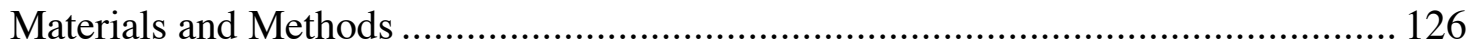

Generation of recombinant GAS superantigens................................................. 126

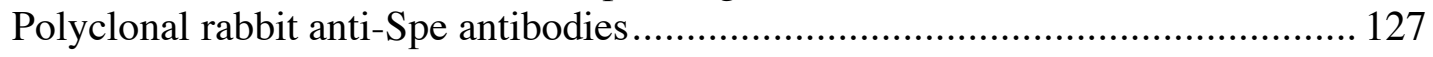

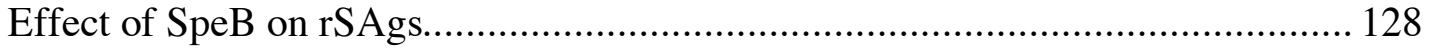

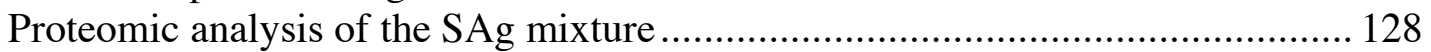

$\mathrm{N}$-terminal sequencing of the Strep SAg following incubation with rSpeB .......... 129

Bioinformatics analysis of SAg sequence .......................................................... 129

Effect of SpeB on the functional activity of streptococcal SAgs........................... 130

Analysis of TCR V $\beta$ repertoire by flow cytometry.............................................. 130

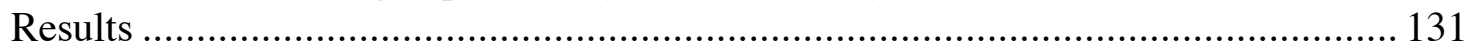

Proteolytic degradation of individual SAgs by SpeB ......................................... 131

SpeB-driven proteolytic degradation of SAgs affects their biological activity ...... 134

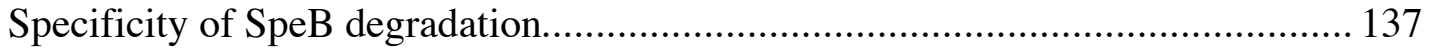

Bioinformatics analysis reveals a putative SpeB-target loop in SmeZ1 ................ 140

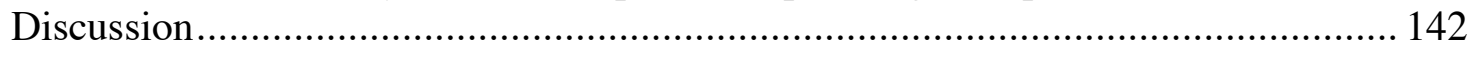

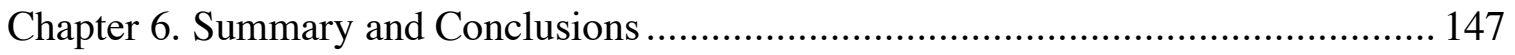

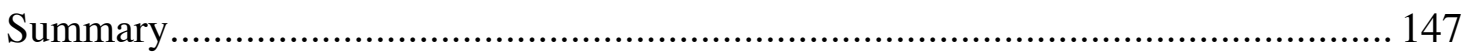

HLA-tg Mice Are Useful Animal Model for Studying SAg-mediated Diseases ....... 149

Relative Contribution of HLA Class II Allelism and TCR V $\beta$ Repertoire

Variation to Strep SAgs Responses.................................................................... 150

HLA Class II Allelic Variation Determines Not Only the Magnitude But Also the

Type of SAg-induced Cytokine Responses ........................................................... 151

Developing a Sensitive System with Minimum Variables for Elucidating the

Molecular Basis of Differential SAg-HLA Class II Interaction ............................... 153

Possible Molecular Mechanisms Underlying the Differential Effect of HLA

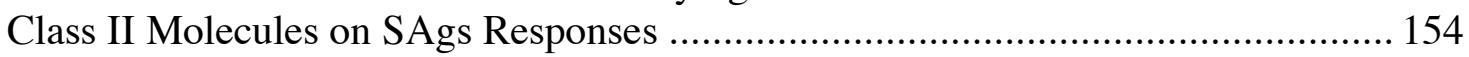

Bacterial Factors Affecting Strep SAgs Responses .................................................. 156

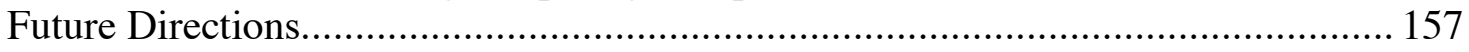

Examine IL-10 as the primary mediator of protective alleles suppressive effects . 157 Study the interaction of protective and high-risk alleles in modulating SAgs responses. 158 
Validate our in vitro findings using HLA-transgenic mice expressing individual or combinations of HLA class II alleles.......................................... 159

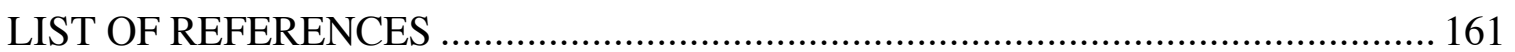

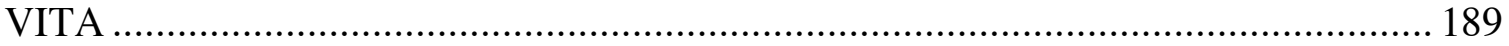




\section{LIST OF TABLES}

Table 1.1 Cell-associated virulence factors ..................................................... 7

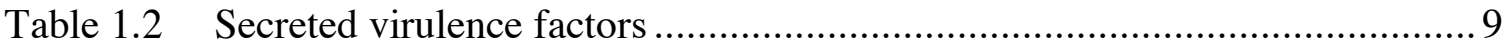

Table 1.3 Biochemical and functional properties of streptococcal SAgs................... 16

Table 2.1 Percentages of splenocytes expressing respective HLA class II transgenes and the mean fluorescence intensity (MFI) of the expression ....57

Table 3.1 Percentages of PBMC expressing HLA DR or DQ and the mean fluorescence intensity (MFI) of the expression .................................. 80

Table 4.1 Primers used to clone different class II alleles and to link the $\boldsymbol{\alpha}$ - and $\beta$-chain by 2 A peptide

Table 4.2 Human B lymphoblastoid cell lines expressing prevalent HLA

class II haplotypes.

Table 4.3 BLS cells transduced with different HLA class II DR or DQ alleles......... 103 


\section{LIST OF FIGURES}

Figure 1.1 Summary of the main GAS virulence factors ........................................

Figure 1.2 MHC-SAG-TCR ternary complexes showing different SAgs groups and distinct modes of SAg-HLA interactions ............................................ 18

Figure 1.3 Ribbon diagram of SEA quaternary complex (group III SAg)................. 19

Figure 2.1 HLA allelic variation affects in vitro response to M1T1 SAgs ..................53

Figure 2.2 Proliferative response of splenocytes from tg and non-tg mice to individual or a mixture of rSAgs produced by the clonal M1T1 strain ....... 55

Figure 2.3 Comparison of the TCR V $\beta$ repertoire of the DQ6 and DR4/DQ8 mice.... 58

Figure 2.4 Cross-presentation of the M1T1 SAgs by APC from DQ6 and DR4/DQ8 mice to each other's T cells reveals that variation in HLA class II is the main controller of the magnitude of SAg.

Figure 2.5 Differential proliferative and cytokine responses of APC-depleted human T cells to native M1T1 SAgs presented by APC from DQ6 or DR4/DQ8 mice

Figure 2.6 Class II allelic variation directly modulates systemic disease severity and survival following infection with M1T1 GAS

Figure 3.1 Proliferative responses of PBMC from individual "A" (protective haplotype) and "B" (risk haplotype) to the native mixture of partially purified M1T1 SAgs

Figure 3.2 T cell responses to the M1T1 SAgs are governed by the type of HLA class II alleles expressed by autologous or allogeneic APC expressing STSS protective or high risk HLA class II haplotypes

Figure 3.3 Comparison of the TCR V $\beta$ repertoire of individual " $A$ " (protective haplotype) and "B" (risk haplotype)

Figure 3.4 The HLA type of the APC determines the type and magnitude of cytokine responses to the Strep SAgs

Figure 3.5 Active suppression of Strep SAgs-driven proliferative responses by IL-10. 
Figure 4.1 Generation of 2A-linked HLA Class II constructs

Figure 4.2 Differential presentation of clonal M1T1 SAgs by prevalent HLA class II haplotypes.................................................................... 108

Figure 4.3 Proliferative response to individual M1T1 GAS rSAgs presented by single class II alleles

Figure 4.4 Cytokine responses to SmeZ1 presented by the indicated HLA DR or DQ alleles.

Figure 4.5 Differential binding of SpeG, SpeJ or SmeZ1 by distinct HLA

Figure 4.6 Calcium mobilization studies

Figure 5.1 Differential degradation of individual rSAgs by SpeB

Figure 5.2 Reproducible differential degradation of mixed rSAgs by SpeB.

Figure 5.3 SAg degradation products are not functionally active 138

Figure 5.4 Sites of cleavage of the various rSAgs by SpeB

Figure 5.5 Ribbon diagram of the 3D structures of SpeA (upper) and SmeZ (lower). 


\section{LIST OF ABBREVIATIONS}

$\mathrm{Ab}$

APC

BLCL

BLS cells

GAS

HLA

IFN- $\gamma$

IL

NF

PBMC

$\mathrm{rSAg}$

SAg

Spe

SmeZ

SSD

STSS

TCR

$\operatorname{tg}$

TNF- $\alpha$
Antibody

Antigen Presenting Cells

B Lymphoblastoid Cell Lines

Bare Lymphocyte Syndrome Cells

Group A Streptococci or Group A Streptococci

Human Leukocyte Antigen

Interferon- $\gamma$

Interleukin

Necrotizing Fasciitis

Peripheral Blood Mononuclear Cells

Recombinant Superantigen

Superantigen

Streptococcal Pyrogenic Exotoxin

Streptococcal Mitogenic Exotoxin Z

Severe Systemic Disease

Streptococcal Toxic Shock Syndrome

T Cell Receptor

Transgenic

Tumor Necrosis Factor- $\alpha$ 


\section{Chapter 1. Introduction}

\section{Group A Streptococci and Human Diseases}

Group A streptococci (GAS), S. pyogenes, are $\beta$-hemolytic, facultative anaerobic, non-motile gram positive, catalase negative, chain forming cocci that normally colonize the skin and throat. However, when the bacteria penetrate the physical barrier of the skin or when the host immune system is compromised, GAS can cause many diseases of variable severity to the extent that they are often considered the most pathogenic member of the genus Streptococcus. GAS are strictly human pathogens with no other natural host $(1,2)$. Streptococci were first differentiated on the basis of hemolytic activity into $\alpha, \beta$, or $\gamma$ hemolytic (3). Subsequently the presence of a specific carbohydrate in the cell wall allowed for further division into groups A, B, C, F, and G (4). Finally, the M- and Ttyping systems were established (5). These systems use serology to identify cell-wallassociated $\mathrm{M}$ and $\mathrm{T}$ protein, respectively. Recently, molecular methods have been used to sequence the $\mathrm{emm}$ gene, which encodes the M protein, where nucleotide differences reflect the specific bacterial emm genotype. With the advent of sophisticated molecular approaches, additional classifications and subclassifications of a given $\mathrm{M}$ serotype or emm genotype can be made taking into account phages that have been acquired by the bacteria (6). Detailed knowledge of the bacterial genome, transcriptome, regulome, lipidome and proteome etc. afforded a deeper insight into molecular mechanisms involved in bacterial virulence and its interaction with genetically distinct host to cause starkly different diseases $(7,8)$. This information allowed us to better understand and dissect host defenses engaged in different diseases caused by these important bacteria. 


\section{GAS pathogenesis}

GAS are among the most versatile of human pathogens; they can potentially infect most human organs or tissues. These bacteria can be carried asymptomatically or cause mild self-limiting superficial illnesses such as pharyngitis, erysipelas and pyoderma. Also, they can invade normally sterile sites where they can initiate severe lifethreatening illnesses such as necrotizing fasciitis (NF) and streptococcal toxic shock syndrome (STSS) or nonsevere invasive disease such as bacteremia and cellulitis, depending mainly on host risk factors (9). In addition to causing these acute diseases, GAS are also associated with the post-infection autoimmune diseases, which may affect the heart, kidney skin, joints, central nervous system or the brain $(10,11)$. These nonsuppurative sequelae of GAS infection include acute rheumatic fever, acute glomerulonephritis, post-streptococcal reactive arthritis or neurological disorders. While most recover with no complications, others suffer chronic and debilitating autoimmune illnesses such as rheumatic heart disease, renal failure or Sydenham Chorea with possible neurologic and neuropsychiatric disorders (12-15). Each year worldwide, GAS cause more than 700 million cases of pharyngitis or skin infection and more than 650,000 invasive infections, with associated mortality of $25 \%$ (16).

\section{Re-emergence of severe invasive GAS infections}

The highly severe forms of acute invasive GAS infections were noted as early as the 1840 's and became a major concern during the 1920s epidemics where they were associated with high fatality rates. During the time between 1920's and late 1970's, the presentation of invasive GAS infections was mostly in the form of mild scarlet fever or 
cellulitis. In the early 1980's, the severe forms of invasive GAS infections suddenly resurged after almost 40 years of decline (17-19). The re-emergence of STSS and NF has been reported in several continents. STSS and NF are rapidly progressive diseases associated with high mortality rates despite prompt antibiotic therapy and surgical interventions. The speed by which the disease progresses in some patients, causing severe necrosis and flesh destruction, lead to the designation of 'flesh eating disease' $(20,21)$.

Although few distinct GAS serotypes, such as M1, M3, M12, and M28, were isolated from severe invasive GAS infections, the M1 serotype has been the most prevalent serotype isolated from both noninvasive and invasive infection cases (17, 22, 23). Interestingly, the resurgence of severe invasive GAS infections coincided with the emergence of a particular subclone of the M1T1 serotype, the clonal M1T1 strain, that has globally disseminated and persisted for more than 25 years as the most prevalent isolate $(24,25)$. Indepth genomic and proteomic studies provided valuable insight into how this virulent M1T1 clone prevails and revealed several features that distinguishes it from related strains. Comparative genomic studies by Aziz et al. (6) showed that the emerged M1T1 clonal strain is distinguished from its ancestral strain by the acquisition of two new prophages encoding important virulence factors; one carries the potent superantigen (SAg), streptococcal pyrogenic exotoxin A (SpeA), and the other carries a novel streptodornase that has the highest activity of all know GAS DNAses $(6,26)$. This strain is also differentiated from its ancestral strain by acquiring from M12 strain a $36 \mathrm{~kb}$ chromosomal segment that carries two important virulence determinants: streptolysin O and $\mathrm{DAD}^{+}$glycohydrolase (NADase), which demonstrated much higher expression and activity than their orthologs present in the ancestral strain but with undetectable activity 
(27). In vivo, this clonal M1T1 strain, unlike its ancestral strain, attains hypervirulence due to a host-imposed selection of mutants in a major two component regulator system $(\mathrm{COVR} / \mathrm{S})$ associated with increased expression and/or preservation of several major bacterial virulence factors, including $\operatorname{Sda1}(28,29)$

In addition, recent studies have revealed novel features of the soluble M1 protein itself that may also contribute to the virulence and prevalence of the clonal M1T1 strain. M1/fibrinogen complexes were shown to induce vascular leakage (30) and M1 protein can also stimulate monocytes via TLR2 to produce high amounts of proinflammatory cytokines (31). Furthermore M1/fibrinogen/M1-specific IgG complexes activate platelets, initiating massive coagulopathy and pro-inflammatory state (32), and soluble M1 protein can act as a SAg thus contributing to the excessive T cell activation and inflammatory cytokines hyper-responsiveness underlying STSS (33). The superantigenic properties of M1 protein may explain the overrepresentation of M1T1 strain among other strains isolated from streptococcal severe invasive cases. Another M proteins (M5) demonstrated SAg-like activities (34), therefore further studies of other M proteins are required to explore whether they possess any of the M1 protein characters.

\section{GAS Virulence Factors}

GAS possess an impressive arsenal of virulence determinants; some are cell associated and others are secreted (Fig. 1.1). These can be functionally subclassified into those involved in bacterial adhesion, invasion of host tissues, evasion of host defenses, and survival. Some bacterial components play overlapping roles in modulating host defenses. Certain virulence factors have been studied in vitro only and their actual role in 


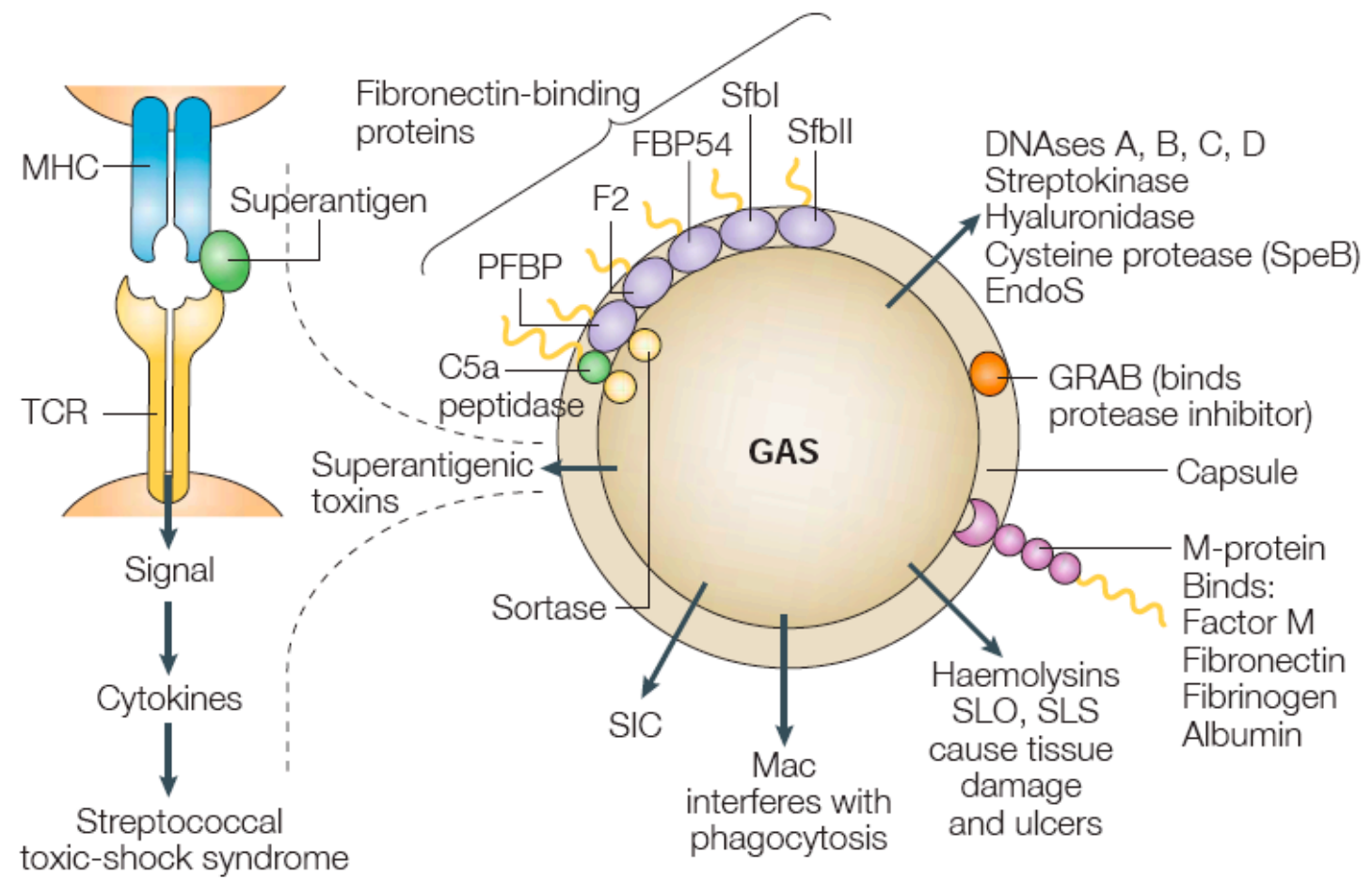

Figure 1.1 Summary of the main GAS virulence factors.

Phagocyte recruitment is reduced by peptidases C5a and SpyCEP that degrade the chemokines C5a and IL-8, respectively; Complement deposition is limited by M protein binding of host counter-regulatory factors $\mathrm{C} 4 \mathrm{BP}$ and $\mathrm{FH}$; phagocytic uptake is reduced by IdeS/Mac-1 binding of phagocyte Fc receptors and the by the $\mathrm{M}$ and $\mathrm{F}$ proteins; resistance to cationic antimicrobial peptides is afforded by D-alanyl modification of cell wall teichoic acid, streptococcal inhibitor of complement (SIC) and streptococcal pyrogenic exotoxin B (SpeB); SpeB also causes bacterial spread; SpeB-mediated degradation of $\mathrm{M}$ protein camouflages the bacteria from recognition by host defenses in the early stages of invasion; SIC is involved in inhibiting complemet-mediated killing; antibody-mediated opsonization is blocked by proteolytic inactivation of $\mathrm{Ig}$ by SpeB, Ides, and endoS; DNase production facilitates escape from neutrophil extracellular traps, and the pore-forming cytolysins SLS and SLO exhibit lytic activity against host neutrophils and macrophages. (Adapted with permission from Mitchell TJ (2003) (35). The Pathogenesis of Streptococcal Infections: From Tooth Decay to Meningitis. Nat rev microbiol 1, 219-230.) 
pathogenesis in not yet clear. I will focus on the well-established virulence factors.

\section{Cell-associated virulence factors}

Cell-associated virulence factors play a role in bacterial adherence (e.g. M protein, fibronectin-binding proteins (FBP), lipoteichoic acid), invasion into host cells and tissues (e.g. hyaluronic acid (HA) capsule, FBP and M protein) or evasion of host immune responses (e.g. M protein, C5a peptidase, HA capsule and SpeB) (Table 1.1). Some of these factors are released in a soluble form from the bacterial surface and can still contribute to pathogenesis (e.g. M protein and glyceraldehyde 3-phosphate dehydrogenase).

\section{Secreted virulence factors}

Secreted virulence factors include degrading enzymes such as proteases and DNases, leukocidal toxins such as the streptolysins $\mathrm{O}$ and $\mathrm{S}$, proteins that inhibit complement activation and interfere with phagocytosis and killing (e.g. streptococcal pyrogenic exotoxin B (SpeB), EndoS, streptococcal inhibitor of complement (SIC) and immunoglobulin G degrading enzyme of S. pyogenes), proteins that promote dissemination (e.g. streptokinase, SpeB, DNase and hyaluronidase), proteins that suppress chemotaxis (e.g. C5a peptidase) and superantigens (SAgs) that induce systemic inflammatory responses and thus increase blood supply of nutrient for the bacteria (Table 1.2). Inasmuch as SAgs are central to our studies, the following section will present a detailed account of their properties, interaction with host cells and their role in certain disease manifestations particularly toxics shock syndrome. 
Table 1.1 Cell-associated virulence factors.

\begin{tabular}{|c|c|}
\hline Virulence factor & Mode of action \\
\hline Lipoteichoic acid & $\begin{array}{l}\text { - Adhesin in initial binding process (36) } \\
\text { - Resistance to neutrophil killing and the antimicrobial peptides } \\
\text { (AMP) through change in D-alanine content (37). }\end{array}$ \\
\hline $\begin{array}{l}\text { Hayluronic acid } \\
\text { capsule }\end{array}$ & $\begin{array}{l}\text { - Resistance to phagocytic killing by serving as a physical } \\
\text { barrier restricting access of phagocytes to the opsonins ( } 38) \text {. } \\
\text { - Plays a role in adhesion by binding to collagen (39) and in } \\
\text { invasion by binding to host hayluronic acid receptor, CD } 44 \text {, on } \\
\text { pharyngeal epithelial cells and inducing cytoskeleton } \\
\text { rearrangement (40). }\end{array}$ \\
\hline $\begin{array}{l}\text { Fibronectin- } \\
\text { binding proteins } \\
\text { (SfbII, Fbp54, M } \\
\text { protein, SOF and }\end{array}$ & $\begin{array}{l}\text { - Adhesins for binding host tissues. Protein F (prtF1, sfbI) also } \\
\text { binds Fc region of IgG, preventing antibody-dependent cell } \\
\text { cytotoxicity by macrophages ( } 41) \text { and promote internalization } \\
\text { into epithelial cells }(42) \text {. }\end{array}$ \\
\hline Pfbp) & $\begin{array}{l}\text { - Bound fibronectin forms complex with collagen that generate } \\
\text { large bacterial aggregates, which in turn mask opsonic epitopes } \\
\text { and protect from phagocytosis }(43) \text {. }\end{array}$ \\
\hline C5a peptidase & - Cleaves the complement-derived chemoattractant C5a (44). \\
\hline $\begin{array}{l}\text { S. pyogenes cell } \\
\text { envelope } \\
\text { protease } \\
\text { (SpyCEP) }\end{array}$ & $\begin{array}{l}\text { - Inhibits neutrophil recruitment and activation by degrading IL- } \\
8 \text {, granulocyte chemotactic protein } 2(\mathrm{GCP}-2) \text { and growth- } \\
\text { related oncogene } \alpha(\mathrm{GRO} \alpha)(45,46) \text {. }\end{array}$ \\
\hline $\begin{array}{l}\text { HtrA serine } \\
\text { protese }\end{array}$ & $\begin{array}{l}\text { - Resistance to oxidative and thermal stress (47). } \\
\text { - Chaperone for SpeB and possibly other secreted virulence } \\
\text { factors }(48,49) \text {. }\end{array}$ \\
\hline M-protein & $\begin{array}{l}\text { - Inhibits complement-mediated opsonophagocytosis by } \\
\text { impeding classical pathway (sequester the complement } \\
\text { regulatory protein C4b binding protein from human serum }(50) \\
\text { and alternative pathway (bind complement regulator factor H) } \\
(51,52) \text {. } \\
\text { - Resistance to neutrophil intracellular killing by inhibiting } \\
\text { phagosome maturation (53). } \\
\text { - Tissue adhesion and internalization }(54,55) \text {. } \\
\text { - Camouflage bacteria and impair IgG-dependent complement } \\
\text { activation by binding IgG through Fc region (56). }\end{array}$ \\
\hline $\begin{array}{l}\text { M-related } \\
\text { protein }\end{array}$ & - Like M-protein, they are antiphagocytic (57). \\
\hline $\begin{array}{l}\text { Collagen-binding } \\
\text { protein }\end{array}$ & - Acts as adhesin (58). \\
\hline $\begin{array}{l}\text { Heme-binding } \\
\text { protein }\end{array}$ & $\begin{array}{l}\text { - Supply iron by acquiring and transporting heme from human } \\
\text { blood (59). }\end{array}$ \\
\hline
\end{tabular}


Table 1.1 (continued)

\begin{tabular}{|c|c|}
\hline Virulence factor & Mode of action \\
\hline $\begin{array}{l}\text { Collagen-like } \\
\text { proteins }\end{array}$ & $\begin{array}{l}\text { - Adherence to epithelial cells and may act as a mask concealing } \\
\text { the bacteria from immune surveillance (60). } \\
\text { - Bind low density lipoproteinns (61). } \\
\text { - Interact with integrin and thus enhance internalization and } \\
\text { reemergence from epithelial cells (62). }\end{array}$ \\
\hline $\begin{array}{l}\text { Lipoprotein of } \\
\text { S. pyogenes } \\
\text { (LSP) }\end{array}$ & - Involved in adhesion and internalization (63). \\
\hline $\begin{array}{l}\text { CD15s-related } \\
\text { antigen }\end{array}$ & - Adhesin and bacterial disguise from immune recognition (64) \\
\hline $\begin{array}{l}\text { Serum opacity } \\
\text { factor (SOF) }\end{array}$ & - Adhesin (65). \\
\hline $\begin{array}{l}\text { Strep leucine- } \\
\text { rich protein }\end{array}$ & $\begin{array}{l}\text { - Evasion of innate immune response by inhibiting phagocytosis } \\
\text { (66). }\end{array}$ \\
\hline Protein $\mathbf{H}$ & $\begin{array}{l}\text { - Inhibits complement activation on bacterial surface by binding } \\
\text { IgG-Fc element (67). }\end{array}$ \\
\hline $\begin{array}{l}\text { Glutathione } \\
\text { peroxidase }\end{array}$ & $\begin{array}{l}\text { - Mediates resistance to phagocyte intracellular killing by } \\
\text { allowing adaptation to oxidative stress (68). }\end{array}$ \\
\hline $\begin{array}{l}\text { Protein G-related } \\
\alpha 2 \text {-M-binding } \\
\text { protein (GRAB) }\end{array}$ & $\begin{array}{l}\text { - Binds the human protease inhibitor } \alpha 2 \text {-macroglobulin to } \\
\text { protect important virulence factors from degradation by either } \\
\text { the streptococcal or host proteases }(69) \text {. }\end{array}$ \\
\hline $\begin{array}{l}\text { Plasmin(ogen) } \\
\text { binding proteins }\end{array}$ & $\begin{array}{l}\text { - Gylceraldehyde-3- phosphate dehydrogenase, } \alpha \text {-enolase and } \\
\text { plasminogen binding group A streptococcal M-like protein } \\
\text { (PAM) aid in the dissemination by breaking down fibrin barriers } \\
(70,71) \text {. } \\
\text {-Shedded glyceraldehyde-3-phosphate dehydrogenase can bind } \\
\text { and inactivate the chemoattractant } \mathrm{C} 5 \mathrm{a}(72) \text {. }\end{array}$ \\
\hline
\end{tabular}


Table 1.2 Secreted virulence factors.

\begin{tabular}{|c|c|}
\hline Virulence factor & Mode of action \\
\hline $\begin{array}{l}\text { Streptokinase } \\
\text { (Ska) }\end{array}$ & $\begin{array}{l}\text { - Converts plasminogen to the proteolytically active plasmin that } \\
\text { accumulates on the bacterial surface and contributes to bacterial } \\
\text { dissemination }(73,74) \text {. }\end{array}$ \\
\hline EndoS & $\begin{array}{l}\text { - Impairs opsonophagocytosis by hydrolyzing conserved } \\
\text { oligosaccharide from IgG preveting its recognition by phagocyte } \\
\text { Fc receptors thus blocking classical complement activation (75). }\end{array}$ \\
\hline Hyaluronidases & $\begin{array}{l}\text { - Breaks down hyaluronic acid in host connective tissues allowing } \\
\text { the organism or its toxins to spread (76). }\end{array}$ \\
\hline DNases & $\begin{array}{l}\text { - Degrade neutrophil extracellular traps (NETs) that capture and } \\
\text { kill bacteria (77). }\end{array}$ \\
\hline $\begin{array}{l}\text { SpeB } \\
\text { (GAS major } \\
\text { cysteine } \\
\text { protease) }\end{array}$ & $\begin{array}{l}\text { - Cleaves IgG, IgM, and IgA impairing antibody-mediated } \\
\text { opsonization (78). } \\
\text { - When retained on bacterial surface, it degrades AMP LL-37 (79). } \\
\text { - Activates matrix metalloproteases, releases biologically active } \\
\text { IL-1 } \beta \text { and kinins from their precursors and degrades vitronectin } \\
\text { and fibronectin contributing to tissue damage and promoting } \\
\text { bacterial spread (80-83). }\end{array}$ \\
\hline $\begin{array}{l}\text { Immunoglobulin- } \\
\text { G degrading } \\
\text { enzyme of } S \text {. } \\
\text { pyogenes (Ides/ } \\
\text { Mac-1) }\end{array}$ & $\begin{array}{l}\text { - Cleaves IgG, preventing phagocytosis }(84) \text {. } \\
\text { - Binds CD16 on neutrophils inhibiting phagocytosis and } \\
\text { generation of reactive oxygen species }(85) \text {. }\end{array}$ \\
\hline $\begin{array}{l}\text { The } \\
\text { streptococcal } \\
\text { inhibitor of } \\
\text { complement } \\
\text { (SIC) }\end{array}$ & $\begin{array}{l}\text { - Inhibits complement-mediated killing by binding the membrane } \\
\text { attack complex C5-7 (86). } \\
\text { - Binds and inactivates the AMP } \alpha \text {-defensins and LL-37 (87). } \\
\text { - Resistance to neutrophils internalization (88). }\end{array}$ \\
\hline $\begin{array}{l}\text { Leukocidal } \\
\text { toxins }\end{array}$ & $\begin{array}{l}\text { - Streptolysin S (SLS) and Streptolysin O (SLO) exert cytocidal } \\
\text { activity on neutrophils and confers resistance to phagocytic killing } \\
(89,90) \text {. } \\
\text { - SLO is required for NADase transport into epithelial cells (91). }\end{array}$ \\
\hline $\begin{array}{l}\text { Sperantigenic } \\
\text { Streptococcal } \\
\text { pyrogenic } \\
\text { exotoxins (Spes) }\end{array}$ & $\begin{array}{l}\text { Disrupt specific immune responses. They interfere with the } \\
\text { adaptive immune response by driving profound Th- } 1 \text { and } \\
\text { suppressing Th- } 2 \text { responses thereby preventing production of } \\
\text { specific cytotoxic antibodies. Also, they may cause T cell anergy } \\
\text { or deletion of T cells bearing SAg-specific V } \beta \text { elements }(92,93) \text {. }\end{array}$ \\
\hline SlaA & $\begin{array}{l}\text { - Phospholipase A2 that promote epithelial adhesion, colonization, } \\
\text { tissue destructions and dissemination (96). }\end{array}$ \\
\hline SibA & $\begin{array}{l}\text { - Binds } \operatorname{IgA}, \operatorname{IgG} \text { and } \operatorname{IgM} \text {; interfering with immune system } \\
\text { recognition of the bacteria (94). }\end{array}$ \\
\hline
\end{tabular}


Table 1.2 (continued).

\begin{tabular}{ll}
\hline Virulence factor & \multicolumn{1}{c}{ Mode of action } \\
\hline $\begin{array}{ll}\text { NAD } \\
\text { glycohdrolase }\end{array}$ & - Inhibits chemotaxis by interfering with actin function (95). \\
\begin{tabular}{ll} 
(NADase) & - Induces epithelial cells apoptosis promoting deeper tissue \\
\hline
\end{tabular} & invasion (91). \\
\hline
\end{tabular}




\section{Superantigens' Structure, Function and Interaction with Immune Receptors}

Superantigens (SAgs) are potent $\mathrm{T}$ cell mitogens that, at concentrations as low as $0.1 \mathrm{pg} / \mathrm{ml}$, can elicit uncontrolled stimulation of resting $\mathrm{T}$ cells resulting in fever, shock, and death (97-99). Because of this feature, SAgs are considered major culprits in GAS severe systemic diseases (SSD) (100-102). The best characterized SAgs are the staphylococcal enterotoxins (SEs), which include staphylococcal entertoxin A to E (SEA to SEE), SEG to SER, SEC-ovine, SEC-bovine, SEC-canine, SEU as well as toxic shock syndrome toxin 1 (TSST-1) (103-105) and the streptococcal SAgs that include several streptococcal pyrogenic exotoxins (Spes) such as SpeA, SpeB, SepC, SpeF, SpeG, SpeH, SpeI, SpeJ, SpeK, SpeL, SpeM, streptococcal SAg A (SSA), and streptococcal mitogenic exotoxin $\mathrm{Z}$ (SmeZ) $(100,106)$. The following section presents an overview of the general characteristics of staphylococcal and streptococcal SAgs as well as SAgs from other related bacteria.

For many years before the mode of action of SAgs was discovered, several laboratories were noticing proteins with unusual biological activities that were produced by S.aureus, S. pyogens, and Mycoplasma arthriditis with potent mitogenic and/or enterotoxic activity (107-110). These proteins, now designated SAgs, include several SEs and Spes. It was in the 1950s that the first bacterial SAg was discovered as a toxin produced by S. aureus and was given the name staphylococcal entertoxin A (SEA) due to its powerful enterotoxic activity (111). However, it was not until many years afterwards that Philippa Marrack and John Kappler elucidated the mechanism of immune stimulation by SEs and were the first to introduce the term 'superantigens' to describe proteins with exceptionally high potency to stimulate human $\mathrm{T}$ cells through 
simultaneous interaction with both HLA class II molecules, normally involved in the presentation of processed antigenic peptides, and with variable elements within the $\beta$ chain of the $\alpha / \beta$ T cell receptor (TCR) (112). To be described as a SAg, a protein has to meet three main conditions: (1) interacts reproducibly with specific TCR V $\beta$ elements (each SAg has a unique V $\beta$ signature); (2) depends on professional antigen-presenting cells (APC) expressing either autologous or allogeneic class II molecules (lack MHC restriction) to elicit potent $\mathrm{T}$ cell proliferation and inflammatory cytokine responses; (3) does not require complex processing by APC to be recognized by T cells.

\section{Conventional antigens (Ag) versus superantigens (SAg)}

There are major differences between SAgs and regular antigens (Ags). Although both bind to major histocompatibility complex class II (MHC II) molecules and to the T cell receptor (TCR), their mode of binding is quite different. Conventional Ags must be processed into small peptides before being loaded onto the MHC molecules, and the processed antigenic peptides are recognized by specific $\mathrm{T}$ cell receptors in the context of self MHC II molecules $(100,103,113)$. In contrast, SAgs bind as intact molecules to MHC II expressed on professional antigen presenting cells (APC), outside the Ag peptide-binding groove, and interact with the TCR at a site different from where regular $\mathrm{Ag}$ is bound $(100,103)$. Recognition of Ag by the TCR involves the combinatorial effect of 5 variable elements: $\mathrm{V} \beta, \mathrm{D} \beta, \mathrm{J} \beta, \mathrm{V} \alpha$, and $\mathrm{J} \alpha$. SAgs, on the other hand bind to specific elements within the variable region of the T cell receptor $\beta$ chain $(\mathrm{TCR} V \beta)(100,103)$.

The human TCR repertoire comprises 25 types of V $\beta$ elements known also as V $\beta$ families. Each SAg has a characteristic TCR V $\beta$ spectrogram, i.e. a characteristic set of 
TCR V $\beta$ elements that it interacts with, and this can range from 1-9 V $\beta$ elements, depending on the SAg $(100,103)$. Depending on how many TCR V $\beta$ s a given SAg interacts with and on the relative frequency of expression of these $\mathrm{V} \beta$ elements in a given person's TCR repertoire, a SAg can activate anywhere from 5-20\% of the resting T cell compared to only $0.00001 \%$ that would be stimulated by a conventional $\mathrm{Ag}(100,103)$. This remarkable ability of SAgs to stimulate such a high numbers of T cells is unmatched by any other physiologic $\mathrm{T}$ cell stimulus, and if uncontrolled can cause serious pathology in the host.

\section{Discovery of streptococcal superantigens}

Before the completion of $S$. pyogenes genome sequence, the gold standard for discovering Strep SAgs was by demonstrating the T cell-stimulating activity of bacterial culture supernatant from which they were purified and functionally characterized.

Showing that a molecule is mitogenic and showing that it is superantigenic are two different things. Malak Kotb and Mark Tomai were the first to provide evidence that GAS possess SAgs by demonstrating their interactions with HLA-II and their TCR V $\beta$ specificity; they showed that pepM5 (M5 protein prepared by controlled pepsin digestion of M5 GAS) was a SAg and also characterized its TCR V $\beta$ specificity and the TCR V $\beta$ of other SAgs produced by GAS $(34,114)$. Although the superantigenicity of the pepM5 protein was disputed and thought to be contaminated by Strep SAgs (115), recent data with M1 protein indicate that it does act as a SAg (33).

Another streptococcal toxin, the scarlet fever toxin or toxin A was suggested to be the cause of scralet fever-like manifestations (Dick reaction) noticed following injection 
of filtered GAS cultures into healthy volunteers (116). Erythrogenic toxin B and toxin C were also identified in GAS culture supernatant (117). As pyrogenic activity was supposed to be the main effect of GAS toxins, these toxins were designated streptococcal pyrogenic exotoxin A (SpeA), SpeB, and SpeC (118). Other Strep SAgs identified experimentally are SSA and SmeZ. A consistent concern during the pre-genomic methods of superantigen identification was that SAg preparations were not all on the same level of purity, which lead to discrepancy in literature. For example, some studies showed that SpeB promoted T cell activation $(119,120)$ while others that used very highly purified SpeB argued that the initial findings were caused by SpeB contamination with unknown SAg (121). SpeB is regarded as the major GAS cysteine protease, and its superantigenic identity is still disputed. The general belief is that its expression in an unnatural host may not allow proper protein folding by HtrA, a GAS chaperon (48) thus affecting its biological functions.

The publication of the first $S$. pyogenes genome sequence marked the start of the post-genomic age of GAS SAgs discovery (122). Mining the DNA database for genes with family signature motifs shared by almost all SAgs lead to identification of several new genes encoding putative SAgs. The predicted genes were cloned, and recombinant proteins were used for functional studies. Novel SAgs were discovered and designated SpeG, SpeH, SpeI, SpeJ, and SpeM (123-125). The rapid discovery of new SAgs caused confusing nomenclature with the same SAg being given two different names. For example, SpeK discovered in the US is the same as SpeL found in Japan and New Zealand (126-128). 


\section{Superantigens produced by other bacteria}

In addition to the well recognized SEs and Strep SAgs, other bacteria have been shown to produce SAgs that are implicated in infection pathogenesis. Group C streptococci (GCS) are regarded as commensals; however, several reports demonstrated their association with severe diseases (129-131). SAgs were found in two different GCS. The Streptococcus equi pyrogenic exotoxins (Sepe) H, I, L and M are homologous to their corresponding S. pyogenes pyrogeinc exotoxins (Spe) H, I, L and M (128, 132). Another two SAgs have been identified from $S$. dysgalactiae subsp. equisimilis called $S$. dysgalactiae derived mitogen SDM and $\mathrm{SpeG}^{\text {dys }}$ that are most similar to SpeM and SpeG, respectively $(133,134)$.

Some Gram-negative bacteria were found to produce SAgs. Yersinia pseudotuberculosis produces the $Y$. pseudotuberculosis mitogens (YPM) a, b and c, and Mycoplasma arthritidis produces the $M$. arthritidis mitogen (MAM). Interestingly, these Gram-negative SAgs differ from staphylococcal or Strep SAgs in many aspects including their TCR V $\beta$ spectrum, their amino acid sequences and the absence of the conserved SAg signature sequence (135-138).

\section{Structural features of SAgs}

SAgs of both S. pyogenes and S. aureus are soluble non-glycosylated molecules, most of which are 20-30kDa proteins. The majority of currently known Strep SAgs have been well characterized with respect to their structure, biological activity, and V $\beta$ specificity (Table 1.3) $(9,100,103,123,124,128,139-141)$. The crystal structure of several Strep SAgs (SpeA, SpeC, SpeH, SpeJ, SSA, and SmeZ-2) has been solved 
Table 1.3 Biochemical and functional properties of streptococcal SAgs.

\begin{tabular}{|c|c|c|c|c|c|c|}
\hline $\mathrm{SAg}$ & $\begin{array}{c}\mathrm{MW} \\
(\mathrm{kDa})\end{array}$ & $\begin{array}{l}\text { Crystal } \\
\text { Structure }\end{array}$ & $\begin{array}{c}\text { HLA II } \\
\text { binding } \\
\alpha / \beta \\
\text { chain }\end{array}$ & $\begin{array}{c}\text { Zinc } \\
\text { dependent } \\
\text { binding }\end{array}$ & $\begin{array}{c}\text { Chromosome } \\
\text { /Phage } \\
\text { encoded }\end{array}$ & $\begin{array}{c}\text { Human TCR V } \beta \\
\text { specificity }\end{array}$ \\
\hline SpeA & 26 & + & $+/-$ & - & $-/+$ & $2.1,12.1,14.1,15.1$ \\
\hline $\mathrm{SpeB}^{\mathrm{a}}$ & 28 & - & $?$ & $?$ & $+/-$ & 8 \\
\hline SpeC & 24.4 & + & $-/+$ & + & $-1+$ & $2.1,3.2,12.5,15.1$ \\
\hline $\mathrm{SpeF}^{\mathrm{b}}$ & 25.4 & - & $?$ & - & $-/+$ & $2,4,8,15,19$ \\
\hline SpeG & 24.6 & - & $-/+$ & + & $+/-$ & $\begin{array}{l}2.1,4.1,6.9,9.1 \\
12.3\end{array}$ \\
\hline SpeH & 23.6 & + & $-/+$ & + & $-/+$ & $2.1,7.3,9.1,23.1$ \\
\hline SpeI & 26 & - & $-/+$ & + & $-/+$ & $5.3,6.9,9.1,18.1,22$ \\
\hline SpeJ $^{\mathrm{c}}$ & 24.6 & + & $-/+$ & + & $+/-$ & $2.1,8$ \\
\hline $\operatorname{SpeK}^{\mathrm{d}}$ & 27.4 & - & $-1+$ & + & $-/+$ & $1.1,5.1,23.1$ \\
\hline SpeL & 26.2 & - & $-/+$ & + & $-/+$ & $1.1,5.1,23.1$ \\
\hline SpeM & 25.3 & - & $-/+$ & + & $-/+$ & $1.1,5.1,23.1$ \\
\hline SmeZ1 & 24.3 & - & $-/+$ & + & $+/-$ & $2.1,4.1,7.3,8.1$ \\
\hline SmeZ2 & 24.1 & + & $-/+$ & + & $+/-$ & $4.1,8.1$ \\
\hline SSA & 26.9 & - & $+/-$ & - & $-/+$ & $1.1,3,15,17$ \\
\hline
\end{tabular}

${ }^{a}$ SpeB is the major Strep cysteine protease; however, its mitogenicity is debated.

${ }^{\mathrm{b}} \mathrm{SpeF}$ is identical to DNase B (142). This molecule contains two separate domains, one responsible for DNase activity and the other involved in mitogenic activity. There is a dispute as to the superantigenicity of SpeF $(143,144)$.

${ }^{\mathrm{c}} \mathrm{M} 3$ and M18 serotypes lack speJ gene suggesting that it is present in a less stable chromosomal region (145)

${ }^{\mathrm{d}}$ SpeK found in M3 strain from US is identical to SpeL identified in M3 strain from Japan and M89 from New Zealand (122, 126, 127). 
(141, 146-149). Despite highly different primary amino acid sequences, SAgs show notable resemblance in their overall structure. Most SAgs show a conserved 3D structure built on two domains: a smaller $\mathrm{N}$-terminal $\beta$-barrel domain, which is homologous to the oligosaccharide/oligonucleotide-binding fold (OB-fold) observed in other bacterial proteins and a larger $\mathrm{C}$-terminal $\beta$-grasp motif separated from the other domain by long $\alpha$-helix (103, 146, 148, 150). Such conserved folding implies an evolutionary pressure to preserve similar architecture and hence capacity to interact TCR and class II MHC (151).

Based on structural features and distinct modes of interaction with immunoreceptors, SAgs may be separated into five different groups $(152,153)$. TSST-1 and TSST-ovine are the only members of group I. These toxins have unique amino acid sequence, but fold into the common SAg structure (154). TSST-1 exclusively binds HLA class II $\alpha$-chain through the $\mathrm{N}$-terminal, low-affinity OB-domain in a peptide-dependent manner (Fig. 1.2A) (155, 156). Group II includes staphylococcal SAgs (SEB, SEC, SEG and SEU) and Strep SAgs (SpeA and SSA) that contain cysteine loop of variable length (10-19 amino acids). Similar to TSST-1, these toxins use the N-terminal low-affinity domain to interact only with the class II $\alpha$-chain; however, unlike TSST-1, in a peptideindependent manner (Fig. 1.2B) (157). Group III contains staphylococcal entertoxins A, $\mathrm{D}, \mathrm{E}, \mathrm{N}, \mathrm{J}, \mathrm{H}, \mathrm{O}$ and $\mathrm{P}$ that are able to cross-link class II molecules by engaging class II $\alpha$-chain through the $\mathrm{N}$-terminal low-affinity site and class II $\beta$-chain by a C-terminal high-affinity zinc-dependent site in the $\beta$-grasp motif of the SAg (Fig. 1.3) (158-160). These toxins have cysteine loop that is exactly 9 amino acids long. Group IV includes Strep pyrogenic exotoxin C, G, J, K, L, M and SmeZ. Similar to group III, group IV SAgs possess the C-terminal high-affinity domain, 
(a)

TSST-1 (Group I)

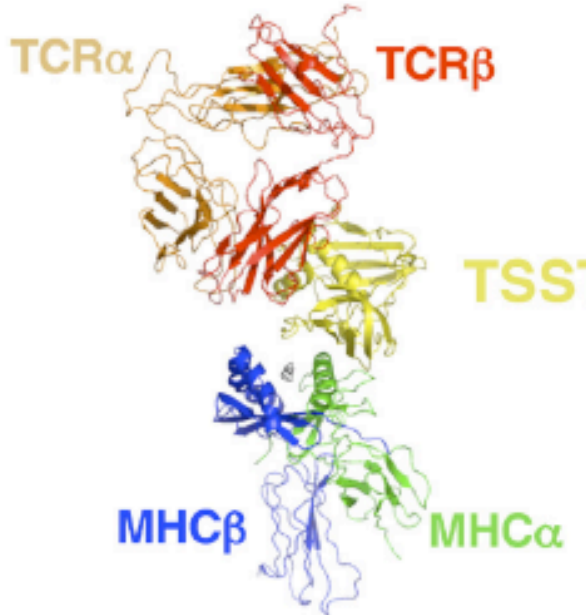

(c)
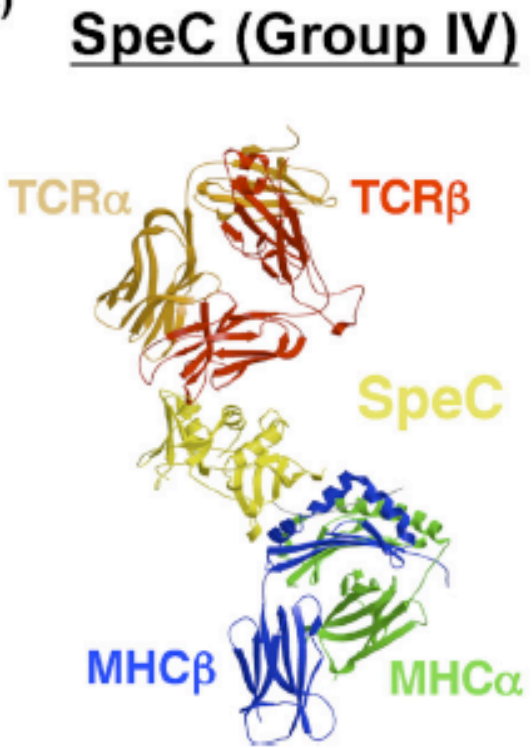

(b)

SEB (Group II)

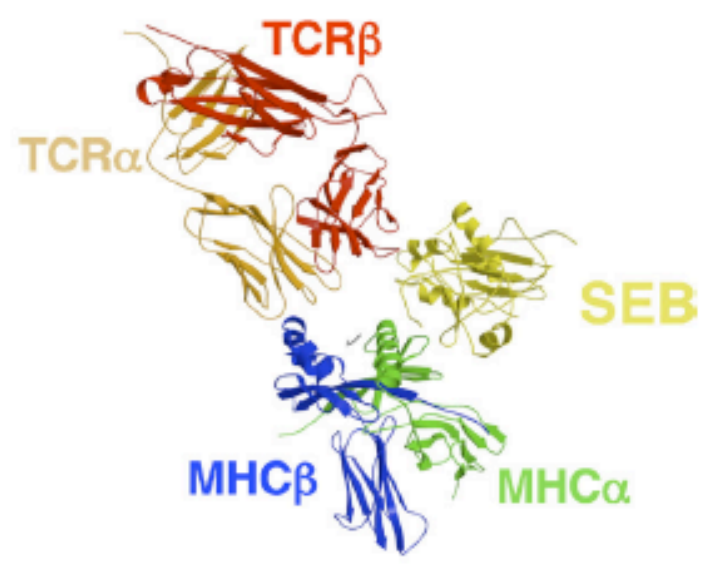

(d)
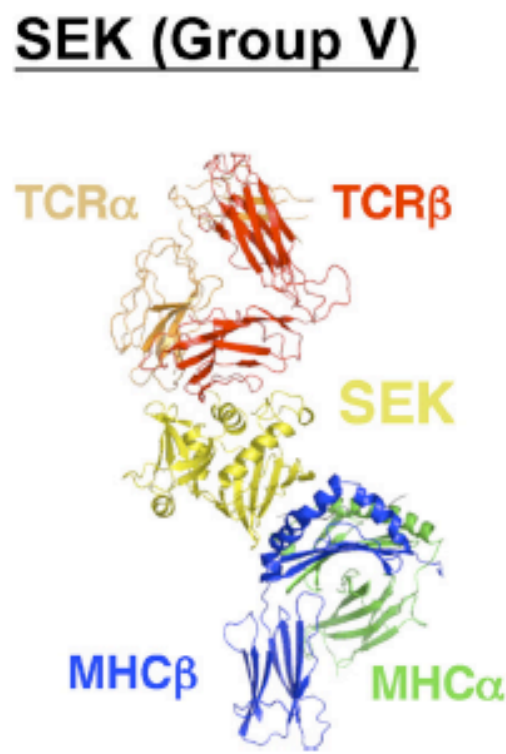

Figure 1.2 MHC-SAG-TCR ternary complexes showing different SAgs groups and distinct modes of SAg-HLA interactions.

Tenary complexes mediated by $A$. TSST-1, $B$. SEB, $C$. SpeC, and $D$. SEK. Colors are as follows: MHC $\alpha$ subunit, green; MHC $\beta$ subunit, blue; antigenic peptide, gray; TCR $\alpha$ chain, orange; TCR $\beta$ chain, red; SAGs, yellow. For clarity, the MHC-SAGTCR complexes mediated by (c) SpeC and (d) SEK are rotated approximately $90^{\circ}$ clockwise about the vertical axis of the page relative to those mediated by (a) TSST-1 and (b) SEB. (Adapted with permission from Günther S. et al. (2007) (161). A Novel Loop Domain in Superantigens Extends Their T Cell Receptor Recognition Site. J Mol Biol 371, 210-221.) 


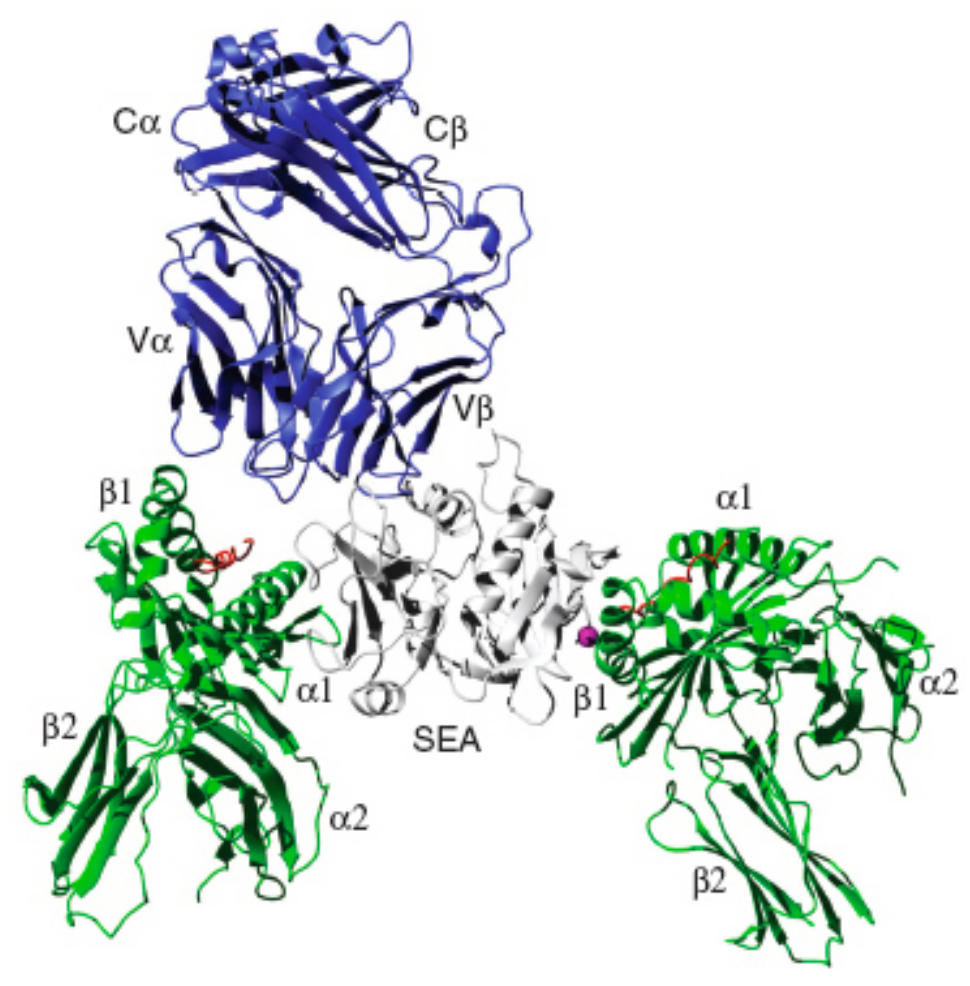

Figure 1.3 Ribbon diagram of SEA quaternary complex (group III SAg).

Crosslinking HLA class II $\alpha / \beta$ chains by SEA (group III SAg). Model of the two HLA (green)-SEA (white)-TCR (blue) quaternary complex. The complex has been produced by superposition of the SAgs in the crystal structure of SEA ${ }_{\text {D227A }}-H L A-D R 1$ (160) representing the low-affinity-binding site and the crystal structure of SEH-HLA-DR1 (159) representing the high-affinity-binding site. (Adapted with permission from K. Petersson, G. Frosberg and B. Walse (2004) (150). Interplay Between Superantigens and Immunoreceptors. Scand J Immunol 59, 345-355.) 
but lack the N-terminal low affinity class II binding site and the cycteine loop (Fig 1.2C) (162). Group V contains SEI, SEK, SEL, SEM, SEQ and SpeI that analogous to group IV, bind HLA class II through C-terminal domain high-affinity site (Fig 1.2D) (163); however, group V SAgs, are characterized by having 15 amino acids loop extension that is absent in other groups and is critical for $\mathrm{T}$ cell activation $(153,161)$

Some Strep SAgs exhibit allelic variation. For SpeA, six alleles have been described. SpeA1, SpeA2 and SpeA3 differ by single amino acid while SpeA4 differs from SpeA1 by about 11\% (164). Also, four synonymous and one nonsynonymous mutations differentiate speA5 differs from speA4, and one synonymous mutations distinguishes speA6 from speAl (165). SmeZ distinctively exhibits much greater variation. SmeZ2 differs by approximately $8.1 \%$ from the very first published SmeZ protein, now known as SmeZ1 (124). Twenty-two additional smeZ alleles were identified through analysis of $37 \mathrm{GAS}$ isolates (166). The functional activities of SmeZ were not affected by such wide allelic variation. However, these smeZ variants showed antigenic differences as indicated by highly variable neutralization responses of human sera (166).

\section{Interactions of SAgs with HLA class II molecules}

Co-crystallization and mutagenesis studies revealed that SAgs have two distinct sites for engaging HLA class II molecules, one at the N-terminal domain called the generic or low affinity binding sit $\left(\mathrm{K}_{\mathrm{D}} \sim 10^{-5} \mathrm{M}\right)$ that mediates class II $\alpha$-chain binding and the other at the C-terminal domain known as the high affinity site $\left(\mathrm{K}_{\mathrm{D}} \sim 10^{-7} \mathrm{M}\right)$, which mediates class II $\beta$-chain binding. The latter is dependent on $\mathrm{Zn}^{2+}$, which is held in a coordination complex via a His (H81) from HLA class II $\beta$-chain and three residues 
(two His and one Asp) from the SAg $(103,152)$.

To ensure they concentrate onto the surface of antigen presenting cells (APC) at the interface with T cells, SAgs have developed several ways for binding HLA class II, which is regarded as a critical aspect of SAgs function. SAg-HLA class II interactions can be categorized into three distinct modes. Mode I involves the SAg N-terminal low affinity region and a site on the $\alpha 1$ domain of HLA class II $\alpha$-chain that entails extensive interactions with the bound antigenic peptide and reaches to make contacts with class II $\beta$-chain as in the case of group I SAgs (TSST-1) (155). This binding mode creates two protein-protein interfaces, which are SAg-HLA and SAg-TCR and prevents any direct contact between HLA and TCR (Fig 1.2A). HLA class-II bound antigenic peptide can influence the presentation of SAgs that utilize mode I $(167,168)$. Mode II is exemplified by group II SAgs that bind through the low affinity N-terminal domain to a site on HLA class II $\alpha$-chain that is completely marginal to the bound antigenic peptide and thus blocks that bound peptide from affecting SAg responses $(157,168)$. Such binding mode creates three protein-protein interfaces namely SAg-HLA, SAg-TCR and HLA-TCR and precludes any contact between TCR and HLA-bound antigenic peptide (Fig 1.2B). Additionally, TCR-HLA contact acts cooperatively to enhance the weak HLA-SAg interaction to a degree sufficient for T cell activation (169). In the last mode, the SAg Cterminal high affinity site interacts in a zinc dependent manner with the $\beta 1$ domain of class II $\beta$-chain and contacts the bound antigenic peptide as illustrated by group IV and V SAgs $(162,163)$. Like mode I, there is no direct TCR-HLA contact and two interfaces exist: HLA-SAg and SAg-TCR and the class-II associated antigenic peptide may influence SAg binding (Fig 1.2C\&D) (162). Importantly, such high affinity of SAg-HLA 
interaction can compensate for poor SAg-TCR binding and allow HLA-SAg-TCR complex to attain efficient $\mathrm{T}$ cell activation (170).

SAgs that are able to crosslink HLA class II molecules on APC induce much more potent immune responses as they up-regulate cytokines and co-stimulatory molecules expression by APC (171-173). SAgs also exhibit different ways for crosslinking class II antigens. For example, SEA cross-links class II molecules by binding one $\alpha$-chain and one $\beta$-chain, and disruption of any of these interactions detrimentally affect T cell activation (173). Both interactions seem to allow SEA to attain the appropriate orientation for engaging certain TCR V $\beta$ elements (174). Other SAgs cross-link class II by forming SAg-homodimers as in case of SED and SpeC; however, through distinct dimerization mechanisms. SED forms $\mathrm{Zn}^{2+}$-stabilized homodimers through C-terminal domains and engages two $\alpha$-chains $(171,175)$ while $\mathrm{SpeC}$ forms homodimers through the N-terminal OB-fold domain and interact with two $\beta$-chains $(139,148)$. Furthermore, other SAgs demonstrate dimer formation; however, the biological relevance of such structural arrangement is not clear. For example, SpeJ crystal structure showed dimer formation. However, the dimerization was through the proposed TCR-binding face, implying two possible functions - normal class II and TCR binding or in the dimer mode, cross-linking class II molecules (141). Additionally, SSA was reported to exhibit monomeric and dimeric forms that occur through intermolecular cysteine bridge involving Cys 26 (176). Note worthily, dimer formation would block TCR interaction, which was reflected in the much higher activity of monomer. Such observation requires additional studies to clarify the role of dimer formation in SSA function. Analogously, crystal structure of SpeA1 dimer involved cysteine bridge between monomeric forms Cys 
90 (177). Even though the dimerization interface involved the TCR binding site, both forms were equally active in vitro, which suggested that flexibility of the dimer interface may permit different orientations than those observed in the crystal structure and thus allow TCR binding.

SAgs vary in their requirement to interact with HLA class II for recognition by and stimulation of T cells, and it appears that both the SAg and the TCR V $\beta$ subset play a role in HLA-independent T cell activation. Kotb and co-workers' studies showed that the requirement for class II-interaction could be bypassed if $\mathrm{T}$ cells receive appropriate costimulatory signals $(178,179)$ with TCR V $\beta$ specificity preserved in case of pepM5 (180) and altered in case of SEA (181). Moreover, SEE unlike SEA is entirely reliant on HLA for T cell activation, though; these SAgs are highly homologous (182). It seems that SAg-T cell direct interaction depends on TCR V $\beta$ elements affinity for the SAg, For example, V $\beta 8$ T cells fail to respond to SEB but not SEC due to much lower affinity for the former (183).

HLA class II isotypic and allelic variation can affect SAg responses. Distinct SAgs demonstrate differential binding and presentation by different class II alleles or isotypes that consequently influences biological reactions to the SAgs and explain the diversity of clinical manifestations observed in individuals infected with the same SAgproducing organism (7). This aspect of HLA-SAg interaction will be discussed later under HLA polymorphism and SAg response. HLA class II associated peptide adds another layer of complexity to Class II-SAg interaction. The presentation of a given SAg by a distinct class II allele can be deeply affected by the characteristics of HLA-bound peptide $(167,184)$ as well as by co-factors involved in peptide antigen presentation such 
as invariant chain and DM (185). As mentioned above this effect may be mediated through TSST-1 interaction with class II-associated peptide (167). However, even for SAgs that do not make contacts with the bound peptide such as SEA similar effects were observed $(184,186)$, which may be explained by the HLA-TCR interaction in the HLASAg-TCR ternary complex. The effect of class II-bound peptide on SAg presentation was suggested to fine tune SAg responses by limiting the number of class II molecules that can bind and present the SAg and thus induce optimal T cell activation through low density and sustained signaling rather than much stronger and shorter signals induced if many more class II molecules were allowed to interact with the SAg $(184,187)$

\section{Interaction of SAgs with TCR}

SAgs stimulate $\mathrm{T}$ cells through specific interaction with particular $\mathrm{V} \beta$ elements of the TCR $(188,189)$. Therefore, SAgs are known to have a distinct TCRV $\beta$ signature. This explains the ability of SAgs overcome the usual specificity between MHC and TCR and activation of all $\mathrm{T}$ cells expressing the SAg-specific TCRV $\beta$ regardless their antigenic specificity. The TCRV $\alpha$ region may to some degree influence the SAg activation of T cells. However, this is probably an indirect effect caused by HLA class II $\beta$-chain interaction with TCR $\alpha$-chain (Fig. 1.2B). SAgs' affinities for TCRV $\beta$ are comparable to those for pMHC-TCR complexes (190). This allows relatively fastdissociation similar to that of pMHC-TCR complexes, which implies that T cell activation by SAGs may also involve serial triggering.

The different groups SAgs (mentioned above) also engage distinct hypervariable (HV) elements and complementarity determining regions (CDR) of TCRV $\beta$ domain. 
TSST-1 (group I SAg) mainly interacts with CDR2 and the third framework region (FR3) (191). SEB and other group II SAgs primarily engage CDR2 and HV4 in a manner that depends mainly on the general conformation of the CDR2 and involves hydrogen bonds with the TCRV $\beta$ backbone atoms (192). Little is known about group III SAgs interaction with TCRV $\beta$. SpeC, similar to other group IV SAgs, interacts with all TCRV $\beta$ hypervariable motifs including HV4, CDR1, 2, and 3. Additionally, abundant side chain hydrogen bonds are involved in SpeC-TCRV $\beta$ interaction. Group V SAgs (e.g. SpeI, SEI and SEK) engages CDR2 and FR3. Also, through their unique 15 amino acids loop, these SAgs interact with FR4 (161). It seems that SAg-TCR interaction specificity is achieved through increased side chain hydrogen bonds formation, engaging an enlarged set of hypervariable elements and contacting rare elements of TCRV $\beta$ region. Also, binding to CDR2 appears to be required for all SAg-TCR interaction, and the percentage contributed by CDR2 to SAg-TCR interface is the largest compared to that of any other CDR or FR.

In contrast to the accepted paradigm that SAgs interact with TCR primarily through the $\mathrm{V} \beta$ domain, $\mathrm{SEH}$ activate $\mathrm{T}$ cells in a $\mathrm{V} \alpha$-specific manner expanding distinct TCRV $\alpha$ elements $(193,194)$. This finding demonstrates the significance of distinct modes of SAg-TCR interaction for a wider activation of T cells and the importance of having diverse SAgs to induce SAg response. Also, demonstrating skewed TCRV $\beta$ repertoire in a patient as an evidence for SAg-mediated disease may not be conclusive.

\section{TCR-generated signaling response to SAgs}

Originally, SAg-induced TCR signaling has been suggested to use the same pathway employed conventional antigen activation (195) that involves activation of CD4 
associated Tyrosine kinase (TK) Lck through dephosphorylation of the inhibitory tyrosine residue by CD45 followed by its recruitment to TCR/CD3 complex (196). However, several lines of evidence suggest that SAg-triggered TCR signaling may involve an alternative pathway, where the use of HLA class II molecules as the primary receptors for SAg would entail CD4+ T cells to constitute the majority of responding $\mathrm{T}$ cells; however, several studies have indicated that $\mathrm{CD} 8+\mathrm{T}$ cells also respond to some SAgs $(197,198)$, which suggests that CD4 is not essential co-receptor for SAg responses. Additionally, monoclonal antibodies blocking of CD4 and using CD4- T cell lines did not affect some SAgs responses $(199,200)$. Therefore, CD4 independent T cell responses to SAgs implies that Lck is also dispensable. This premise was supported by the discovery that some SAgs can stimulate T cells lacking Lck function $(201,202)$. By the use of various kinase inhibitors and Lck-defective T cells, it was found that the Lckdependent and -independent pathways meet at the ERK-1/2 activation step and that such $\mathrm{T}$ cells can mobilize $\mathrm{Ca}^{2+}$ in response to SEE stimulation but fail to respond when protein kinase C (PKC) was inhibited (200). Since phospholipase C- $\gamma 1$ (PLC- $\gamma 1)$ is inhibited in the absence of Lck and it is the PL involved in the typical TCR-signaling trigerred by petide-HLA, it was suggested that a different PL is required for the alternative pathway utilized by SAgs. Indeed, It was found that PLC- $\beta$ (which is regulated by the G $\alpha-11$ family of G proteins) is involved in the SAg alternative signaling pathway. These data may help clarify certain SAg responses such as the ability of CD8 T cell to respond to some SAgs despite SAg recognition in the context of HLA class II molecules. Also, it was suggested that unlike Th2, Th1 responses are Lck-independent $(203,204)$, which explains the observed preferential SAg induction of Th1 cytokines $(205,206)$. 
Another aspect of T cell activation is the role of co-stimulation. Early work by Kotb and co-workers provided useful insights into co-stimulatory signals in $\mathrm{T}$ cell responses to SAg stimulation. It was shown that SAg and mitogen (e.g. phytohemagglutinin, PHA) induced distinct biochemical events during T cell activation (207) and that T cell responses to SAgs required both secreted and cell associated factors $(178,208,209)$, where the latter was shown to be delivered by CD28 cross linking (179, 210). Ohnishi et al. characterized the CD28 co-stimulatory pathway in SAg-mediated T cell activation (210). They were the first to provide direct evidence for the involvement of PKC in this pathway, and also showed that it involved PLC- $\gamma 1$ activation and an instantaneous sustained rise in intracellular $\mathrm{Ca}^{2+}$ level. Interestingly, response sensitivity to cyclosporin A was reliant on the absence (sensitive) or presence (resistant) of phorbol myristate acetate, which suggested that $\mathrm{Ca}^{2+}$-dependent or -independent responses may be produced based on the co-stimulus character. Therefore, CD28 co-stimulation boosts SAg-triggered TCR signals by promoting the activation of PLC- $\gamma 1$ and PKC in a cyclosporine A-sensitive pathway that is distinct from alternative SAg-induced TCR signaling pathway. On the other hand, B7 interaction with cytotoxic T lymphocyteassociated antigen-4 (CTLA-4) dampened SAg-induced T cell activation $(211,212)$, suggesting that CTLA-4 regulatory activities are operative in SAg-TCR signaling.

\section{Superantigens in Human Disease}

The unusual immune responses triggered by SAgs are believed to be responsible for the pathogenesis of SAg-associated diseases. These molecules can interact with a large spectrum of $\mathrm{T}$ cells and induce excessive inflammatory cytokines production. SAgs 
have been implicated in the pathogenesis of several human diseases such as toxic shock syndrome (TSS), and autoimmune diseases $(213,214)$.

\section{Food poisoning}

Food poisoning is an intoxication caused by consumption of SEs produced in food contaminated by toxin-producing staphylococci. Emesis is the primary symptom of this illness. The mechanism of SE-induced emesis is not clear because gastrointestinal tract receptors have not been yet discovered (215). An intact cysteine loop, mentioned above under structural features, is required but not sufficient for the emetic response because SpeA has this loop but is not emetic (216). Furthermore, the mitogenic activity of SE appears to be separable from the enterotoxic behavior $(215,217)$.

\section{Staphylococcal toxic shock syndrome (staphTSS)}

StaphTSS is a severe illness that involves multiple organs. StaphTSS manifestations include hypotension, diffuse rash, fever, vomiting, diarrhea, and skin desquamation upon recovery (152). Not all staphylococcal SAgs are equally important in the production of TSS. Menstrual TSS is mainly caused by STSS-1 (109), whereas nonmenstrual TSS can be triggered by TSST-1 and other staph SAgs particularly SEB and SEC $(218,219)$. Support for the involvement of these toxins in non-menstrual TSS comes from their induction of TSS-like symptoms in animal models (99, 220). Interestingly, SEB, SEC, and TSST-1 interact only with class II $\alpha$-chain through the low affinity site, and thus are expected to be less potent than SAgs that interact with class $\beta$-chain high affinity site. However, higher levels of these toxins may be produced in vivo (105). 


\section{Streptococcal toxic shock syndrome (STSS)}

STSS, caused by S. pyogenes, is the most severe form of invasive streptococcal infections, with about $50 \%$ or more mortality rates. Although STSS shares many symptoms with staphTSS, there are important differences, which include disseminated infections, severe pain, tissue invasion, and bacteremia are usually associated with STSS (221, 222). Several lines of evidence support the involvement of Strep SAgs in STSS. First, the in vivo pattern of TCR V $\beta$ repertoire expression in STSS patients was skewed, reflecting V $\beta$ specificities of SAgs produced by the infecting GAS isolates (102). Also, passive administration of anti-Strep SAgs neutralizing antibodies to STSS patients significantly alleviated disease severity and reduced mortality $(223,224)$. Importantly, direct evidence was obtained from studies that demonstrated circulating SAgs in patients suffering from STSS (225), and SAgs expression in tissue biopsies from STSS cases together with increased infiltration of CD68 and CD4 cells producing high levels of TNF$\beta$ and IFN- $\gamma$, which are typical of a SAg-induced response (101). Additionally, the direct ability of Strep SAgs to cause STSS has been confirmed in experimental models (226, 227).

The pathogenesis of staphTSS and STSS is believed to result from the abnormal immune responses elicited by SAgs, which are potent inducer of inflammatory cytokine not only from the $\mathrm{T}$ cells bearing SAg-reactive $\mathrm{V} \beta$ elements, but also from the HLA class II APC $(206,228)$. Elevated cytokine production correlates with the physiological changes characteristic of septic shock $(229,230)$. TNF- $\alpha$ and $-\beta$ induce capillary leakage that results in hypotension and multiple organ failure $(213,231)$. TNF- $\alpha$ also promotes the release of other cytokines such as IL- 1, IFN- $\gamma$, and TNF- $\beta$ that potentiate TNF- $\alpha$ 
activity $(232,233)$. IL-1 has pyrogenic activity that causes fever and, at high levels, can trigger hypotension (234). IFN- $\gamma$ upregulates HLA class II expression and thus provide SAgs with more receptors, which potentiates SAg-induced cytokine induction (235).

\section{Kawasaki syndrome (KS)}

KS involves the development of systemic vasculitis that targets primarily young children and is known to be the principal cause of acquired heart disease in children. A growing body of data suggests SAgs involvement KS (236). First, KS shares clinical features and immunological reactions with StaphTSS and STSS (237). Also, early administration of intravenous immunoglobulins is highly effective, which points to a toxin as the causative agent. Importantly, several studies reported selective expansion of certain TCRV $\beta$ subsets, suggesting a possible involvement of $\operatorname{SAgs}(238,239)$. Furthermore, elevated serum IgM specific for several SAgs has been observed in KS patients (240).

\section{Acute rheumatic fever (ARF)}

ARF is a delayed sequel to GAS pharyngitis. Manifestations involve inflammation of several organs such as joints, heart and central nervous system (241). Cardiac rheumatic fever is believed to result from a cross-reactive immune response to the host's cardiac tissue. SAg involvement in ARF was suggested by the observation that several ARF-associated serotypes were shown to harbor numerous Strep SAgs. SpeL gene was frequently found in serotypes M3 from USA and Japan $(126,127)$ and in M89 from New Zealand (128). Also, SpeM gene was found in M18 from USA (242). 
Importantly, sera from convalescent ARF patients showed higher titer of SpeM-specific antibodies than those from pharyngitis patients or healthy individuals (125).

\section{Role of Host Genetics in Infectious Diseases}

\section{Host genetic involvement in the pathogenesis of infectious diseases}

The effect of host genetics on the outcomes of infectious diseases is becoming more and more evident. Predisposition to and protection from infections vary among people exposed to certain pathogens $(243,244)$. Genetic contribution relative to the environmental and other factors is variable. Inasmuch as most infectious diseases have an underlying immunological component, it was not surprising that most genetic associations discovered were related to genes that regulate the immune responses. Extensive genetic polymorphism in immune response genes, especially HLA genes, is critical to the survival of human species because it ensure that some individuals with unique genetic makeup will be able to mount successful immune response to a highly mutable pathogen $(243,244)$. Exploring the immune response and the genetic makeup of susceptible and resistant individuals can shed more light on the disease process.

Additionally, studying host genetic influence on infectious diseases offers new diagnostic tools, advances understanding of case definition and prognosis and induces novel prophylactic and therapeutic approaches.

Variations in immune receptors and associated signaling components, cytokine genes, genes that regulate cellular adhesion and homing patterns as well as genes encoding complement and HLA molecules have been shown to affect susceptibility to 
several infections $(7,245,246)$. This section will discuss such variations and the associated disease outcomes.

It has been shown that Toll-like receptor-2 (TLR2) gene polymorphism is associated with increased risk for leprosy and tuberculosis (247). Similarly, TLR4 variants with lower expression level are associated with Gram -ve bacterial infection and septic shock (248). Furthermore, polymorphism in complement component genes are also linked to increased susceptibility to certain infections. For example, FC $\gamma$ RIIa inefficient variants are linked to severe meningococcal disease susceptibility (249), which is also modulated by variations in mannose-binding lectin (250).

The classically known heterozygous sickle hemoglobin is not the only genetic variation that protects against malaria (251), but also the Duffy blood group that inhibits the expression of Duffy gene encodes a chemokine receptor on red blood cells, which is also the entry gate for $P$. vivax (252). Chemokine receptors variation is also involved in HIV infection; CCR5 $\Delta 32$ deletion variant, a co-receptor for HIV, and CCR2-Ile64 variant are associated with delayed progression to AIDS in heterzygotes and HIVprotection in homozygotes $(253,254)$. Also, variants of RANTES (CCR5 ligand) (255), stromal cell-derived factor-1 (CXCR4 ligand) (256) and IL-10 (257) have been shown to play a role in progression to AIDS.

Cytokines orchestrate the immune responses and their genes polymorphism can affect infections outcomes. Variations in IL-4 and IL-10 have been shown to affect outcomes of hepatitis $\mathrm{C}$ virus $(\mathrm{HCV})$ infections and progression rates of $\mathrm{HIV}$ infections (258-261). Additionally, polymorphisms that promote higher levels of IL-10 production are associated with adverse outcome of pneumococcal infection (262). Interestingly, the 
same genotype (- 592 C) is also associated with hepatitis B virus (HBV) clearance (263). Several single nucleotide polymorphisms were reported in TNF- $\alpha$; some of them are associated with altered secretion that affects sepsis outcome and susceptibility to cerebral malaria, HBV, HCV and leprosy (264-267). Cytokine gene mutations also can cause some otherwise healthy individuals to develop severe mycobacterial infections, though, by weakly pathogenic strains. These individuals have defective IFN- $\gamma$ responses due to variations in IL-12 (268), IL-12 receptor (IL-12 R) (269), or IFN- $\gamma$ R (270). Not only the cytokine genes and receptors do play a role in such mycobaterial infections, but also the signaling pathways play a role where defects in STAT1 pathway involved in IL-12 and IFN- $\gamma$ were reported $(271,272)$.

\section{The same GAS strain is associated with variable infection severity}

It was noticed that individuals infected with GAS strains can develop different clinical manifestations; some suffer from severe sepsis, while others are colonized and never experience disease. Even among invasive cases, some develop NF and STSS; others have a mild bacteremia. This variation in clinical symptoms could be due to differences in the virulence of the infecting strains, differences in host susceptibility or both. We are mainly interested in studying host factors that may contribute to variable susceptibility.

The M1T1 clonal strain was isolated from individuals with starkly different symptoms ranging from uncomplicated pharyngitis to invasive infection cases with or without SSD $(273,274)$. Additionally, the inflammatory responses triggered by SAgs from the same M1T1 clone in different people can vary considerably, and there is a direct 
correlation between the levels of inflammatory cytokine responses and the severity of invasive disease $(101,275,276)$. These observations led to the suggestion that individual variations in immunogenetic factors that regulate SAg responses may be important determinants of infection severity, which is the fundamental hypothesis studied in this dissertation.

\section{Host risk factors for invasive streptococcal infections}

Demographic factors such as age, gender and pre-existing conditions would be expected to affect the clinical outcome of invasive infection. Such factors can affect disease progression or confound case classification and should be carefully considered in the analysis of association studies. Both host and environmental factors can play a role in the susceptibility to invasive GAS infections (277-279). Drug abuse by injection, use of corticosteroid and chronic medical conditions such as diabetes, cancer, cardiovascular diseases and HIV infection are among the host factors, while household size and contact with children suffering sore throat are environmental factors that increase the risk for invasive GAS infections among adults over 18 years old. Furthermore, Varicella zoster virus infection, recent use of non-steroidal anti-inflammatory drugs and crowded household are associated with increased risk for developing invasive GAS infection; however, large home and, surprisingly, exposure to child with rhinitis are associated with decreased risk among children under 18 years old $(278,279)$.

Moreover, pre-existing levels of protective humoral immunity such as opsonic M type-specific antibodies (Abs) and SAgs-neutralizing Abs are critical factors. It was shown that low levels of these Abs were associated with increased risk for invasive 
disease. However, there was no correlation between Ab levels and disease severity as both severe and non-severe cases had equally low levels of protective Abs (273).

\section{HLA association with infectious diseases}

In humans, the major histocompatibility complex is known as the HLA system, which represents the most polymorphic genetic locus in the human genome. It includes three major classes of HLA, class I, class II and class III that are located on chromosome 6 and play an essential role in developing and regulating immune responses to pathogens.

Both HLA class I and class II molecules present antigenic peptides to T cells, which afterwards mount antigen-specific immune responses. The class I molecules are HLA-A, -B and -C, while the class II molecules are HLA-DP, -DQ and -DR. Class I molecules are expressed on all nucleated cells, whereas class II molecules are expressed on professional APC whose primary function is to present processed foreign antigens to CD4 T cells. Both HLA class I and II consist of $\alpha$ and $\beta$ heterodimers. Class I $\beta$-chain, $\beta 2$ microglobulin, is monomorphic, whereas the $\alpha$-chain is highly polymorphic.

Similarly, class II DR $\alpha$-chain is non-polymorphic, while both $\alpha$ - and $\beta$-chains of class II molecules (e.g. DRB1*, DQA1*, DQB1*) are highly polymorphic. Individual genes are referred to by a nomenclature that indicates locus and allelic designation. Thus, polymorphic HLA DR genes encode the DR $\beta$ polypeptide at a locus known as DR $\beta 1$. Individual alleles carry four digit designation that follows the locus name, i.e. DRB1*1501.

The extensive polymorphism in HLA molecules is generally concentrated in the peptide-binding groove, which allows highly diverse peptides to be efficiently bound and 
presented by different HLA molecules. HLA remarkable polymorphism is thought to have evolved primarily through natural selection by pathogens $(280,281)$. Several genes that map to the HLA region have been implicated in a number of infectious diseases. HLA allelic variation has been associated with susceptibility or resistance to malaria, tuberculosis, leprosy, HIV progression, HBV and HCV persistence $(280,282)$.

Reports demonstrated that HLA allelic variation can influence malaria susceptibility where HLA-B53 and HLA DRB1*1302 are associated with protection. Schistosomiasis is another parasitic infection where HLA polymorphism plays a role in the development of post infection hepatic complications, for example HLA-A2 and -B12 are associated with high-risk for S. mansoni-triggered hepatic fibrosis while HLA-DR2 is protective. Susceptibility to mycobacterial infections is also influenced by HLA polymorphism, for example HLA DR2 is associated with increased susceptibility to leprosy and tuberculosis. In case of viral infections, the phenotype influenced by HLA is progression to AIDS in HIV infections and persistence carriage in $\mathrm{HBV}$ and $\mathrm{HCV}$ infection. In HIV infection, HLA-B35 is associated with poor prognosis, whereas HLAB27 is associated with a better one. Similarly, HLA DRB1*1101 and HLA DRB1*1302 are associated with resistance to persistent $\mathrm{HCV}$ and $\mathrm{HBV}$, respectively.

Kotb and co-workers' studies identified significant associations between specific HLA class II haplotypes and different manifestations of GAS sepsis and poststreptococcal rheumatic heart disease (RHD) $(275,283)$.

DRB1*0701/DQB1*02/DQA1*0201 haplotype was associated with increased risk for RHD while DRB1*13/DQB1*0603/DQA1*0103 haplotype was associated with protection (283). In invasive GAS infections, DRB $1 * 1501 / \mathrm{DQB} 1 * 0601$ haplotype is 
associated with strong protection from both STSS and NF, whereas

DRB1*1401/DQB1*0503 haplotype is associated with high risk for STSS. Furthermore, it was found that DRB1*03/DQB1*0201 is protective from NF, while DRB1*11/DQB1*0301 is high-risk for NF (275). This was the first study to demonstrate that HLA polymorphism can influence the outcome of a Gram-positive infection and to present a plausible molecular basis for such association. The findings suggested that HLA class II allelic variation could shape the outcome of SAg-mediated infection through differential SAg-presentation by distinct class II alleles/haplotypes. Also, different class II associations with STSS and NF supported the view that these are two different clinical manifestations with distinct underlying mechanisms and require different interventional approaches.

\section{HLA class II polymorphism and SAg responses}

HLA class II molecules on APC serve as receptors for SAgs that present them to the T cells. Although some SAgs can interact directly with T cells in the absence of HLA class II molecules $(182,183)$, CD28 crosslinking was required to induce T cells to proliferate $(179,180,209,210)$. These observations suggested that the requirement for HLA class II molecules in SAg responses is to bring T cells and APC in close proximity to allow productive interactions between CD28 and B7 molecules as well as other receptors/ligands on both types of cells $(179,180,209,210)$. Therefore, the ability of SAgs to bridge HLA molecules on APC and TCR on T cells is essential for effective intercellular communication and for subsequent generation of biochemical signals and activation of both cell types. 
The presence of more than one class II isotype (DR, DQ, DP) and their extensive polymorphism is expected to influence SAgs binding and presentation and thus their biological activities. Indeed, several studies have shown that HLA class II allelic variation can affect the type of signaling transduced through the MHC and TCR receptors and affect the magnitude of SAg response $(185,284-288)$. These studies support the view that not all class II isotypes or alleles bind or present a given SAg the same way and that the ensuing response depends on both the SAg and the class II allele. While most S.aureus SAgs are presented better by HLA DR than by HLA DQ, streptococcal SAgs vary in their preference for HLA DR or HLA DQ, where SpeA and SpeB are preferentially presented by DQ alleles, whereas SpeC shows superior presentation by DR than DQ alleles (287-289). Thus, Some SAgs are presented better by DR than by DQ and vise versa, and the type of DR or DQ allele can make a big difference. The preferential presentation of different SAgs by distinct class II alleles could be explained in some, but not all, cases by differential binding affinity $(284,288,289)$. The lack of correlation between binding and presentation of some SAgs may be related to the stability of the ternary complex of HLA-SAg-TCR which is derived from the combined strengths of HLA-SAg, TCR-SAg and HLA-TCR interactions. The high affinity of the TCR V $\beta$ subsets to a given SAg may stabilize poor SAg-HLA interaction and thus T cells can react to a particular SAg-HLA combination, even though the later cannot be easily be revealed by simple binding studies compared to much more sensitive functional studies.

The effect of class II allelism on SAg responses was also shown in vivo in experimental models $(226,227,290)$. HLA DQ8 (DQB1*0302/DQA1*0301) transgenic (tg) mice developed SSD and mounted significantly higher responses to SpeA than other 
HLA-tg mice or non-tg mice (227). Direct evidence for the effect of HLA class II allelic variation on $\mathrm{SAg}$ presentation is also demonstrated in results presented in this dissertation and from previous studies done in our lab $(275,288)$.

\section{Specific Aims}

Host genetic factors are important determinant of susceptibility to infectious diseases. GAS can cause a large diversity of diseases ranging from uncomplicated pharyngitis and mild skin infection to NF and STSS caused by excessive inflammatory cytokine release triggered by Strep SAgs that have been shown to play a crucial role in the pathogenesis of such SSD. Thus, GAS offer an excellent model for elucidating the role of host factors in infection. Previous work in our laboratory showed that not all individuals with invasive infection develop SSD and the same GAS strain can be isolated from patients with starkly different clinical manifestations. These observations pointed to a strong involvement of certain host factors in shaping the outcome of GAS infections.

Several host factors may contribute to differences in disease manifestation, particularly HLA class II as these molecules are receptors for the SAgs and their polymorphism influence the disease outcome by controlling the inflammatory response to SAgs. Epidemiological studies showed that specific HLA class II alleles (DRB1*15/DQB1*0602) conferred strong protection from SSD, while others $(\mathrm{DRB} 1 * 14 / \mathrm{DQB} 1 * 0503)$ increased the risk for SSD. This argues strongly for a role of HLA class II allelic variation in contributing to differences in the severity of invasive GAS disease. 
The hypothesis investigated in this dissertation is that the differential presentation of SAgs by protective or high risk alleles is related to variation in the binding affinity or conformational changes resulting from the combined interaction of the tri-molecular complex, composed of the T cell receptor, HLA class II and SAg; this variation may account for the differences in the biochemical signals that follow the bridging of the TCR and HLA class II by the SAg. This hypothesis is supported by the observation that the proliferative and the inflammatory cytokine responses to SAgs differ quantitatively and qualitatively when the same SAg is presented by the protective versus the high risk or even the neutral class II alleles.

The specific aims of this study can be summarized as follows:

1. To use an animal model to validate the finding of our epidemiological studies demonstrating the critical role of HLA class II allelic variation as the primary controller of Strep SAg responses and infection severity.

2. To determine whether the HLA class II effect on GAS SAg responses is related to a specific SAg or a particular mix of GAS SAgs, or it is the same for all GAS SAgs. Some Strep SAgs are more potent than others. It is important to determine which ones are differentially affected by the protective and high-risk alleles because these SAgs are likely to be the ones contributing to SSD. Such studies will help develop a sensitive system with minimum number of variables that can be used for the rest of our studies.

3. To investigate possible molecular mechanisms underlying the differential effect of HLA class II polymorphism on SAgs responses by investigating any differences in the binding affinity of the SAg to the class II alleles or differences in the 
biochemical events that follow the initial interaction of SAg with its receptors on $\mathrm{T}$ cells and APC.

Our study may help more thorough understanding of the effects of HLA class II allelism on the SAg-driven responses and better predicting the outcome of SAgassociated disease in a given patient. Possibly, treatment strategies can be customized depending on patient's HLA class II haplotype. Similar to other clinical conditions, specific interventions can produce more effective results than general therapeutic approaches. For example, the use of IVIG in treating GAS SSD has been reasonably effective in reducing morbidity and mortality $(224,291)$. However, in the US and other parts of the world IVIG is expensive and sometimes unavailable. Our observations could recognize patients at low risk for SSD, thus IVIG can be secured for those at high risk. Also, we will be able to determine if a particular SAg or combination of SAgs can induce the most extreme responses. Such information will be useful at several levels. If a particular SAg or SAgs combination are the main cause for the observed HLA associations and for SSD, thus we can get an explanation for the resurgence of SSD by tracing the introduction of such $\mathrm{SAg}(\mathrm{s})$ before and after 1980 when SSD reappeared. Additionally, therapeutic approaches can be tailored to block this particular SAg or SAgs combination. On the other hand, if all studied SAgs are equivalent, then we will examine other factors that may have played a role in SSD reappearance and develop more generic therapies that block all SAgs activities. 


\section{Chapter 2. HLA Transgenic Mice Provide Evidence for a Direct and Dominant Role of HLA Class II Variation in Modulating the Severity of Streptococcal Sepsis*}

\section{Introduction}

Group A streptococci (GAS) are strictly human pathogens that cause a wide spectrum of diseases ranging from mild skin infections and uncomplicated pharyngitis to very severe life-threatening infections, e.g., streptococcal toxic shock syndrome (STSS) and necrotizing fasciitis (NF) $(221,292)$. In genetically susceptible individuals, GAS infections can sometimes trigger autoimmune disorders such as rheumatic fever, rheumatic heart disease, glomerulonephritis and possibly a number of neurological disorders $(9,100,283,293)$. Since the early 1980 s, there has been a sharp resurgence of severe invasive GAS infections such as STSS and NF (292). GAS produce many virulence factors that contribute to their pathogenesis, but the streptococcal pyrogenic exotoxins (Spes), which belong to the family of microbial superantigens (SAgs), play a crucial role in STSS and NF (100-102, 275, 294). As SAgs, the Spes bind to both MHC class II and TCR molecules triggering very potent inflammatory cytokine responses, which in certain individuals leads to severe systemic diseases (SSD) manifested by STSS and multiple organ failure $(100,275)$. Our previous studies have provided conclusive evidence that GAS SAgs play a pivotal role in the pathogenesis of severe invasive illnesses $(101,102,224,275)$, however; we noted that only a few individuals infected

\footnotetext{
* Adapted by permission of American Association of Immunologists from Nooh MM., El-Gengehi N., Kansal R., David CS., Kotb M. (2007). HLA Transgenic Mice Provide Evidence for a Direct and Dominant Role of HLA Class II Variation in Modulating the Severity of Streptococcal Sepsis. J Immunol 178, 3076-3083.
} 
with SAg-producing GAS develop SSD $(224,274,275)$. One possible explanation for the differences in the severity of invasive disease is the fact that some GAS strains are more virulent than others. Another mutually non-exclusive possibility is that the disease outcome is modulated by host immunogenetic variations.

Although there are over 100 known serotypes of GAS, the resurgence of severe invasive infection coincided with the emergence or re-emergence of virulent strains belonging to ancient serotypes that have apparently acquired genetic material via horizontal transfer $(6,24)$. In particular, a specific subclone of the M1 strain that has disseminated globally, persisting as the most frequently isolated clone from clinical cases over the last 25 years seemed to have acquired two unique prophages, each encoding virulence factors that are not found in the ancestral M1 strain (6). However, we showed that the same M1T1 clonal strain causes starkly different manifestations in different individuals, and was in fact isolated from cases with SSD, STSS, NF, non-severe invasive disease as well as from individuals with uncomplicated pharyngitis (274). Similar findings are observed with other prevalent serotypes, e.g. the M3 serotype (295). These findings provided a compelling argument that despite the resurgence of virulent strains, host factors must be contributing significantly to modulating the outcome of invasive GAS infections.

We and others have shown that the magnitude of inflammatory responses triggered by the same SAg can vary considerably in vitro as well as in vivo in different responders $(275,276,289)$. A logical explanation for these findings is that variations in certain host immunogenetic factors modulate responses to the SAgs and, accordingly the severity of the systemic disease. Indeed, our epidemiological studies demonstrated a 
significant association between specific alleles of the HLA class II and the severity of invasive GAS infection (275). While the haplotype DRB1*15/DQB1*06 (DR15/DQ6) conferred strong protection from SSD, the haplotype DRB1*14/DQB1*05 (DR14/DQ5) was associated with predisposition to SSD and other haplotypes, e.g. DRB1*04/DQB1*0302 (DR4/DQ8) were neutral with respect to the disease outcome. We also showed that inflammatory responses to the M1T1 SAgs, in the presence of the protective haplotype, were much attenuated as compared to the high-risk and neutral haplotypes (275).

To provide an in vivo validation to these findings, we needed an appropriate animal model for SAg-mediated GAS pathogenesis. Animal models have contributed significantly to the knowledge of the mechanisms involved in gram-positive bacterial toxic shock, however, regular mice are much less sensitive to SAg-mediated effects than humans $(99,296,297)$. This difference relates, in part, to the lower binding affinity of SAgs to murine MHC class II as compared to human HLA class II (298). The advent of the HLA class II transgenic (HLA-tg) mice provided an opportunity to study the pathogenesis of GAS in a readily manipulated animal model with a sensitivity to SAgs similar to that of humans $(226,227,299-301)$. Indeed several studies have shown that HLA-tg mice mount potent responses to GAS SAgs $(226,227,290,297,302)$.

In this study, we examined the in vitro and in vivo responses to GAS SAgs in HLA-tg mice expressing HLA-DQ6 (SSD protective allele) or HLA-DR4/DQ8 (SSD neutral alleles). The work presented here provides evidence that HLA class II allelic variation contributes directly to streptococcal invasive disease outcome and confirms our previous epidemiological findings of the protective effect of the HLA-DQ6 allele. 


\section{Materials and Methods}

\section{Transgenic mice}

HLA transgenic, MHC class II deficient $\left(\mathrm{H}-2 \mathrm{Ab}^{\circ}\right)$, mice expressing human DR4/DQ8 and DQ6 alleles were generated in the laboratory of Chella S. David (Mayo Clinic, Rochester, MN)(303-305). Mice were bred and maintained in accordance with the guidelines established by our institutional animal care committee. We confirmed the presence of the appropriate HLA class II transgenes by PCR-based genotyping using the following allele specific oligonucleotide primers: DQ6 sense 5'-AGG ATT TCG TGC TCC AGT TTA AGG CCA TG-3' and DQ6 antisense 5'-TCT GCA AGA TCC CGC GGA ACG CC-3', DQ8 sense 5'-AGG ATT TGG TGT ACC AGT TTA AGG GCA T-

3' AND DQ8 antisense 5'-TGC AAG GTC GTG CGG AGC TCC AA-3', DR4 sense 5'GTT TCT TGG AGC AGG TTA AAC A-3' and DR4 antisense 5'- CTG CAC TGT GAA GCT CTC AC-3'. We also confirmed expression of HLA class II molecules on mice splenocytes by flow cytometry using monoclonal antibodies (mAb) specific to HLA-DQ (FITC, Leu-10, Catalog. No. 347453) or to HLA-DR (L227) followed after washing by FITC-goat F(ab')2 specific to mouse IgG (Accurate Chemical \& Science Corp., Westbury, NY). Analysis was conducted on a FACSCalibur (Becton Dickinson).

\section{Preparation of partially purified streptococcal SAgs from GAS culture supernatants}

We used two representative invasive isolates of the clonal M1T1 strain to prepare a partially purified mixture of the native secreted streptococcal SAgs. Isolate 5622 (isolate A) was from a severe invasive case and isolate 6021 (isolate B) from a non- 
severe invasive case. Previous studies determined that these two isolates are genetically indistinguishable, belonging to the global M1T1 strain (274). The bacteria were streaked on blood agar plates, subcultured in Todd-Hewitt broth (THB) supplemented with 1.5\% yeast extract, and grown overnight at $37^{\circ} \mathrm{C}$ under static conditions. The recovered culture supernatants were mixed with ice-cold absolute ethanol (1:3 v:v) and incubated at $-20^{\circ} \mathrm{C}$ overnight for complete precipitation of the secreted proteins, which include the SAgs. The precipitate was dissolved in distilled water containing $0.5 \mathrm{mM}$ of the protease inhibitor PMSF and subjected to extensive dialysis against deionized water at $4{ }^{\circ} \mathrm{C}$. The dialysate was filter-sterilized with a $0.2 \mu \mathrm{m}$ syringe filter (Sarstedt, Germany) and treated with polymyxin B agarose to remove any traces of endotoxin, as previously described (206). Aliquots of the dialyzed, LPS-depleted and sterilized native mixture of M1T1 SAgs were stored at $-20^{\circ} \mathrm{C}$ until used.

\section{Generation of recombinant GAS superantigens}

Recombinant SpeA2 (rSpeA2), rSmeZ1, rSpeG and rSpeJ were expressed as histidine-tagged fusion proteins according to the manufacturers' recommendations (Promega, Madison, WI. and Qiagen Inc. Valenica, CA). Primer pairs used for the cloning were as follows: SpeA2 (672bp-5'G AGG CCT CAA CAA GAC CCC GAT C 3' forward and 5'G AAG CTT ACT TGG TTG TTA GGT AGA CT3' reverse), SmezI (645bp-5'G AGG CCT TTA GAA GTA GAT AAT AAT TC3' forward and 5'CA AAG CTT AGG AGT CAA TTT C3' reverse), SpeG (630bp-5' T AGG CCT GAT GAA AAT TTA AAAG3' forward and 5' C AAG CTT CTA GTG CGT TTT TAA3' reverse) and

SpeJ (630bp-5' G AGG CCT ATG AAA AGA ATA ATA AAA ACA A 3' forward and 
5' G AAG CTT ATT TAG TCC AAA GGT AA 3' reverse). The forward and reverse primers contained Stuland HindIII restriction sites, respectively. The amplified PCR products were purified, subcloned in pGEM-T Easy vector (Promega Corporation, Madison, WI, USA), and sequenced (UTHSC Molecular Resource Center). The correct cloned inserts were digested with Stul and HindIII, moved to the pQE30Xa vector (Qiagen), propagated in DH5 $\alpha$ Escherichia coli cells and subsequently used to transform M15 E. coli cells. Recombinant SAg (rSAg) protein expression was induced with $1 \mathrm{mM}$ IPTG (isopropyl-b-D-thiogalactopyranoside) for $3 \mathrm{~h}$. Proteins in the bacterial cell lysate were resolved by SDS-PAGE, and the specific expression of each rSAg protein was determined by immunoblotting with RGS-His HRP Conjugate Kit (Qiagen) and specific rabbit polyclonal anti-Spe Ab. rSAgs were purified as His-fusion proteins using Ni-NTA superflow according to manufacturer's recommendations (Qiagen) and their purity was confirmed by resolving the eluted fractions on SDS-PAGE and silver staining to detect any contaminating proteins. The purified rSAgs were treated with polymyxin B agarose (Boehringer Mannhiem, Indianapolis, IN) to remove any contaminating endotoxin. The activity and $\mathrm{V} \beta$ specificity of our rSmeZ1 was found to be comparable to rSmeZ1 that was kindly provided by Dr. Proft (University of Auckland, New Zealand).

\section{T cell proliferation assays}

Spleens were aseptically removed from the HLA-tg mice and dispersed into single-cell suspensions. The cells were treated with $\mathrm{NH}_{4} \mathrm{Cl}$ red blood cells lysis buffer, washed three times in Hank's balanced salt solution (HBSS), and resuspended to $2 X 10^{6}$ cells $/ \mathrm{ml}$ in RPMI 1640 with 5\% (v/v) heat-inactivated FBS, 4 mM L-glutamine, 25 
$\mathrm{mM}$ Hepes, $50 \mathrm{U} / \mathrm{ml}$ penicillin/50 $\mu \mathrm{g} / \mathrm{ml}$ streptomycin, and $50 \mu \mathrm{M}$ 2-mercaptoethanol (RPMI complete medium). The cells were seeded at $2 \times 10^{5} /$ well into U-bottom 96-well microtiter plates (Costar Corp., Cambridge, MA) and stimulated with optimal dilutions/doses of (a) mixture of native secreted M1T1 SAgs, (b) individual rSAgs produced by the clonal M1T1 strain $(0.1-100 \mathrm{ng} / \mathrm{ml} \mathrm{rSpeA2,} \mathrm{rSpeG,} \mathrm{rSpeJ} \mathrm{or} \mathrm{rSmeZ1),}$ or (c) Con A $1 \mu \mathrm{g} / \mathrm{ml}$. After incubation for $72 \mathrm{~h}$ at $37^{\circ} \mathrm{C}$ in a $5 \% \mathrm{CO} 2$ and $95 \%$ humidity, the cultures were pulsed for the final $6 \mathrm{~h}$ with $1 \mu \mathrm{Ci} /$ well $\left[{ }^{3} \mathrm{H}\right]$ thymidine (specific activity $=6.7 \mathrm{Ci} / \mathrm{mmol}$; Dupont, Wilmington, $\mathrm{DE}$ ), harvested onto glass fiber filters, washed, and the $\left[{ }^{3} \mathrm{H}\right]$ thymidine uptake was measured by counting on a Matrix 97 direct ionization $\beta$-counter (Packard Instruments Co., Meriden, CT). All samples measurements were performed in triplicates, and the data were presented as mean cpm $\left[{ }^{3} \mathrm{H}\right]$ thymidine uptake \pm SD. Each experiment was repeated at least three times.

\section{Crisscross SAg presentation assays}

Mouse APC-depleted T cells were purified from splenocytes by negative selection using the mouse pan $\mathrm{T}$ cell magnetic isolation kit and AutoMax according to the manufacturer's instructions (Miltenyi Biotech, Auburn, CA). Pure APC-depleted T cells were cultured in RPMI complete medium, and their purity was confirmed physically by flow cytometry and functionally by the lack of response to stimulation by M1T1 SAgs. Experiments in which pure $\mathrm{T}$ (cultured alone without any APCs) cells showed a response to Con A or SAgs were discarded. We prepared APCs from each HLA-tg mouse strain by treating whole splenocytes with mitomycin C (Sigma) $50 \mathrm{ug} / 5 \times 10^{7}$ cell $/ \mathrm{ml}$ for $20 \mathrm{~min}$ at $37^{\circ} \mathrm{C}$, washing them five times with HBSS, and suspending them in RPMI complete 
medium. The efficacy of mitomycin $\mathrm{C}$ treatment was established by the nonresponsiveness of APCs to stimulation with Con A or M1T1 SAgs. Pure, APC-depleted T cells $\left(1 \mathrm{X} 10^{5} /\right.$ well) from a specific HLA-tg mouse strain were co-cultured in U-bottom 96-well microtiter plates, with either autologous APCs $\left(5 \times 10^{4} /\right.$ well) or with the same number of APCs from the other HLA-tg mouse. Proliferation assays were conducted as described above.

In similar assays, pure human $\mathrm{T}$ cells were used as responders instead of the pure murine $\mathrm{T}$ cells. To prepare human $\mathrm{T}$ cells, we used ficoll hypaque gradient centrifugation to obtain the peripheral blood mononuclear cells (PBMCs) from healthy donors' blood. We purified the T cells from PBMCs by one cycle of erythrocyte rosetting followed by negative selection using the human pan $\mathrm{T}$ cell magnetic isolation kit and AutoMax (Miltenyi Biotech, Auburn, CA). The purity of the APC-depleted T cells was confirmed by flow cytometry and by the lack of response to PHA or the M1T1 SAgs as detailed above.

\section{Cytokine measurement}

Splenocytes were cultured as described under the proliferation assays (see above). Culture supernatants were collected from the individual wells after 24,48 , and $72 \mathrm{~h}$ of incubation, cleared of cells by centrifugation, and stored at $-80{ }^{\circ} \mathrm{C}$ for subsequent analysis. The cytokines IL-2, IFN- $\gamma$, and TNF- $\alpha$ were simultaneously quantified using the mouse cytometric bead array (CBA) kit (Pharmingen, San Diego, CA) according to the manufacturer's instructions. A FACSCalibur (Becton Dickinson) was used to determine the fluorescence intensity and the assay results were analyzed using the $\mathrm{BD}$ 
CBA software (Becton Dickinson, San Jose, CA). The amounts of cytokines produced in $\mathrm{pg} / \mathrm{ml}$ were assessed by comparison to standards run in parallel with the test samples. Where human $\mathrm{T}$ cells were the responding population, we used the human cytokine Lincoplex kit (Linco Research, St Charles, CA.).

\section{Analysis of the $T$ cell receptor $V \beta$ (TCR V $\beta$ ) repertoire by flow cytometry}

Relative quantitative analysis of TCR V $\beta$ repertoire was conducted by flow cytometry using the mouse V $\beta$ TCR screening panel kit (BD Pharmingen, Catalog No. 557004). CD3-PE-Cy5 conjugate (BD Pharmingen Catalog No. 555276) was used as an additional marker to enable proper gating on T cells only. Approximately $5 \times 10^{5}$ splenocyte/tube in $100 \mathrm{ul} \mathrm{PBS}$ containing 1\% BSA were stained with mAbs for different $\mathrm{V} \beta$ according to the manufacturer's instructions. Using a FACSCalibur flow cytometer (Becton Dickinson), we gated on the CD3-PE-Cy5 cells and analyzed individual TCR V $\beta$ per tube. The data were analyzed using FlowJo software (Tree Star, Inc. Ashland, OR 97520). A minimum of 30,000 cell events was acquired for each analysis.

\section{In vivo infection studies}

In preparation for infections, a representative isolate of the clonal M1T1 strain was grown for $17 \mathrm{~h}$ at $37^{\circ} \mathrm{C}$ without shaking in THB supplemented with $1.5 \%$ yeast extract. The bacteria were washed twice in sterile PBS and diluted to the appropriate $\mathrm{cfu} / \mathrm{ml}$ in PBS. DQ6 and DR4/DQ8 mice $(\mathrm{n}=10)$ were infected with $5 \times 10^{6} \mathrm{cfu} / \mathrm{mouse}$ in $250 \mu 1$ PBS bacteria via the tail vein. Mouse survival was monitored twice daily over 7 days. We also assessed the systemic inflammatory responses elicited as a result of the 
GAS infection by collecting heparinized blood $(\sim 150 \mu \mathrm{l})$ at $24 \mathrm{~h}$ post infection from the retro-orbital plexus. The levels of inflammatory cytokines TNF- $\alpha$ and IFN- $\gamma$ were determined as describe above. Bacteremia was determined by plating serial dilutions on blood agar plates. For assessing bacterial load in the organs, infected mice were killed by $\mathrm{CO} 2$ asphyxiation and bacteria were evaluated by preparing organs homogenates in PBS and plating 10-fold serial dilutions on blood agar plates. $\beta$ hemolytic colonies were counted after incubation at $37^{\circ} \mathrm{C}$ for $24 \mathrm{~h}$.

\section{Statistical analysis}

Data are expressed as mean of triplicates or quadruplicates \pm SD. Statistical differences were calculated on original values using the nonparametric Mann Whitney U test. Kaplan-Meier survival curves were generated to compare mice survival following infection and the significant differences in survival curves were calculated by employing the log rank test. A value of $p<0.05$ was considered significant.

\section{Results}

\section{Differential in vitro response of HLA-tg mice splenocytes to M1T1 SAgs}

To determine the effect of HLA class II variation on the response to GAS SAgs, spleen cells from mice expressing different HLA class II alleles were stimulated in vitro with partially purified mixture of native SAgs secreted by two different M1T1 isolates: isolate A from severe invasive STSS case, and isolate B from non-severe invasive case of mild bacteremia. Mice expressing the high-risk haplotype/alleles DR14/DQ5 are not yet 
available and thus we compared mice expressing the protective DQ6 allele to mice expressing the DR4/DQ8 alleles that are neither associated with protection nor with highrisk for SSD, i.e. neutral. As expected, the response to M1T1 SAgs from both isolates was comparable, reflecting the clonality of this strain. However, the proliferative response of splenocytes with DQ6 or DR15 alleles, both of which are present on the protective HLA class II haplotype in our epidemiological studies, was significantly lower $(p<0.001)$ than that of splenocytes expressing the neutral DR4 and/or DQ8 alleles (Fig. 2.1A). These differences were specific to the SAgs response inasmuch as the Con A responses were similar regardless the type of the HLA class II alleles expressed on mice splenocytes (Fig. 2.1A). Splenocytes from the non-tg background mice strains failed to respond to the M1T1 SAgs (data not shown).

In support of the proliferation data, splenocytes from the DQ6 mice produced significantly lower levels of TNF- $\alpha$, IFN- $\gamma$, and IL-2 than those from the DR4/DQ8 mice in response to the same M1T1 SAgs (Fig. 2.1B). Cytokine responses peaked at the $72 \mathrm{~h}$, those of the DQ6 mice were 6, 30, and 60-fold lower than those of the DR4/DQ8 mice for TNF- $\alpha(\mathrm{p}<0.001)$, IFN- $\gamma(\mathrm{p}<0.01)$, and IL-2 ( $<<0.001)$, respectively.

To further confirm that the response is specific to the M1T1 SAgs, we also compared the proliferative response of the splenocytes from the DR4/DQ8 and DQ6 mice to rSpeA2, rSpeG, rSpeG and rSmeZ1, individually as well as in a mixture. The pattern of the response to rSAgs mixture reflected that obtained with the native partially purified mixture of SAgs where the response of the DQ6 splenocytes was consistently and significantly less than that of DR4/DQ8 splenocytes and both responses were higher than the responses of the traditional inbred background mice splenocytes (Fig. 2.2). 
Figure 2.1 HLA allelic variation affects in vitro response to M1T1 SAgs.

In vitro stimulation of spleen cells from various HLA-tg mice with M1T1 SAgs

A. Proliferative responses of the indicated HLA-tg mice to the native mixture of M1T1 SAgs. Spleen cells ( 2 X10 $/$ well) were stimulated in triplicates by an optimal dilution (1:500) of the mixture of partially purified native SAgs secreted by M1T1 streptococcal isolate $\mathrm{A}$ from a severe invasive case, isolate $\mathrm{B}$ from a non-severe invasive case, or by Con A $(1 \mathrm{ug} / \mathrm{ml})$. B. Cytokine responses of splenocytes from the indicated of HLA-tg mice to native M1T1 SAgs mixture. DQ6 or DR4/DQ8 spleen cells were incubated with the SAgs mixture in triplicates, and at the indicated times points the culture supernatant was collected and analyzed for the production of IL-2, TNF- $\alpha$ and IFN- $\gamma$. Each point represents the mean of triplicate $\pm \mathrm{SD}$; shown is a representative of one from at least four independent experiments. $\mathrm{P}<0.01$ * $^{* *}, \mathrm{P}<0.001$ : $^{* * *}$ based on all experiments using the Mann-Whitney U test. 
A

目 DR15 (protective)

- DR4/DQ8 (neutral)

DQ6 (protective)
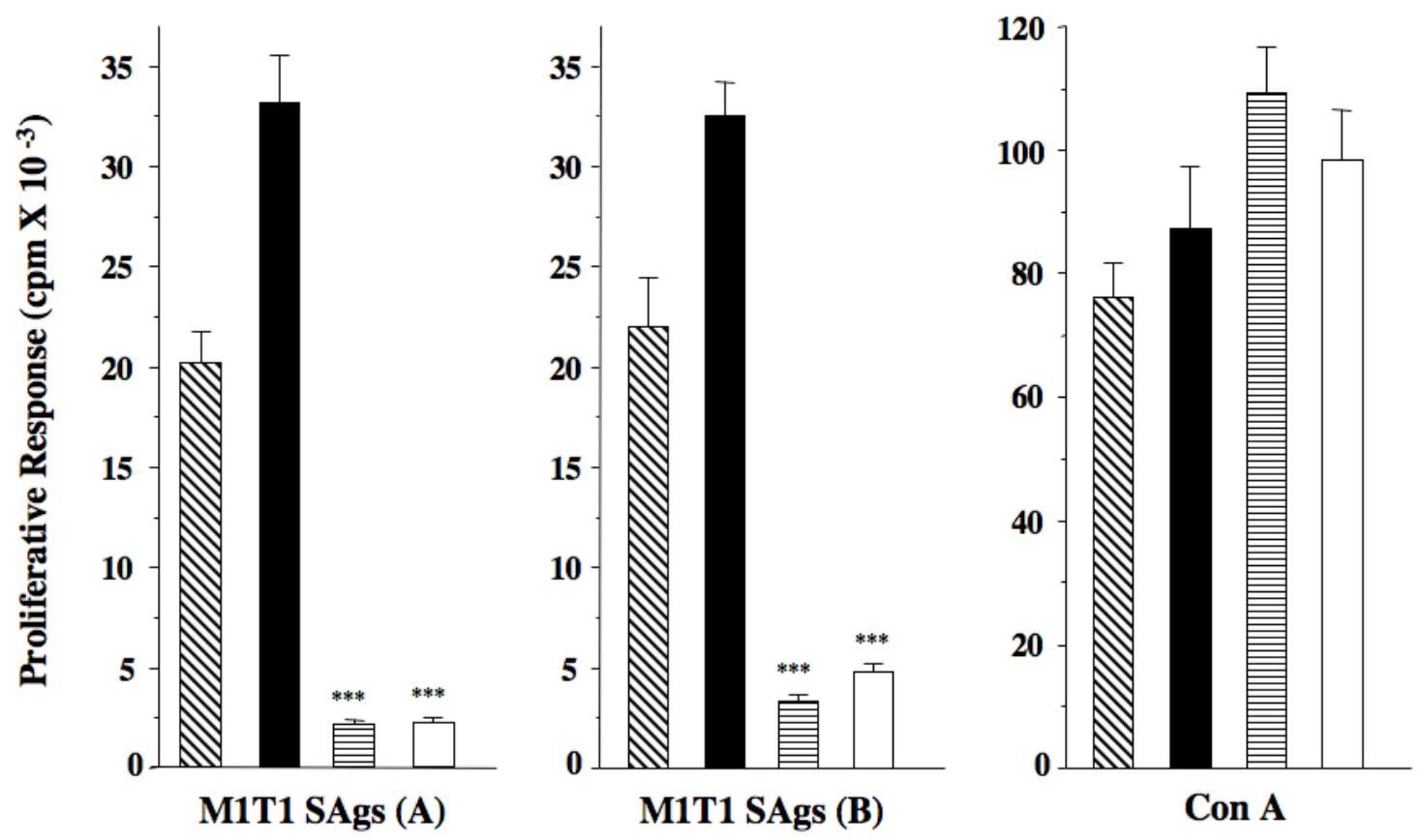

B

- DR4/DQ8 (neutral)

$\square$ DQ6 (protective)
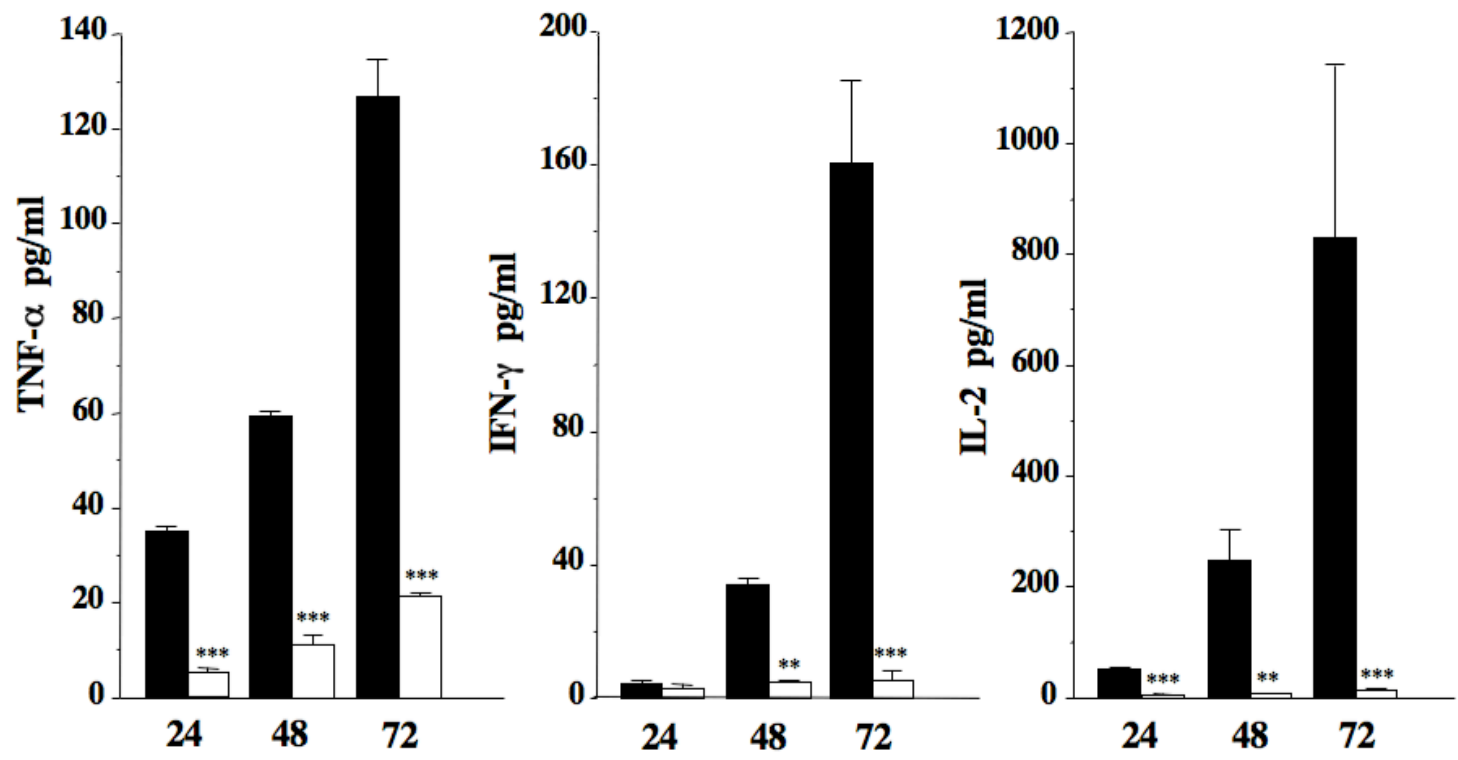

Hours in Culture 

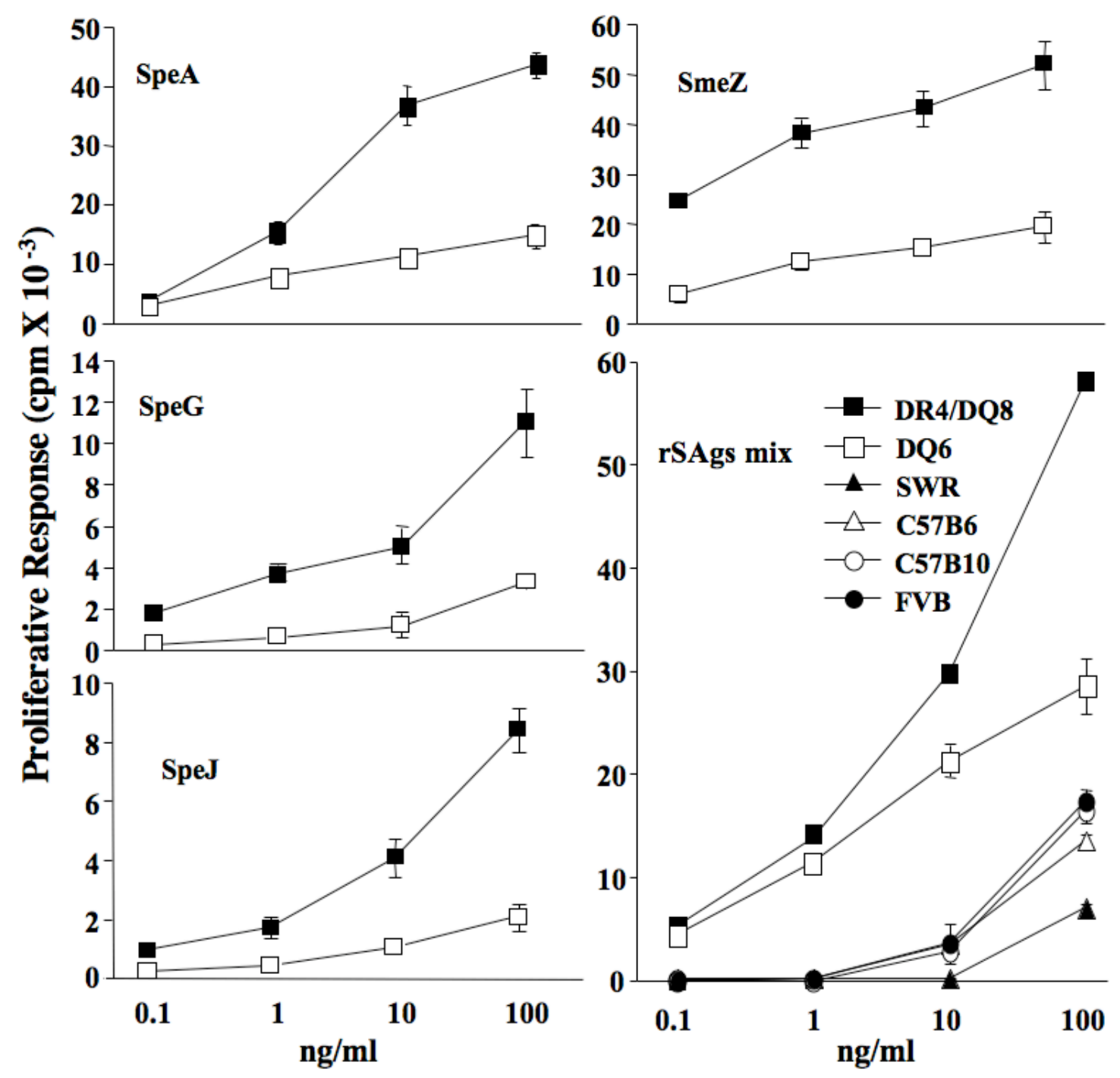

Figure 2.2 Proliferative response of splenocytes from tg and non-tg mice to individual or a mixture of rSAgs produced by the clonal M1T1 strain.

Spleen cells (2X10\% /well) from DQ6 or DR4/DQ8 mice were stimulated with the indicated concentrations of individual $\mathrm{rSAg}(0.1-100 \mathrm{ng} / \mathrm{ml})$ as well as a rSAg mixture. Individual rSAgs were mixed in equal amounts and $0.1-100 \mathrm{ng} / \mathrm{ml}$ of this mixture was used to stimulate mouse spleen cells. Spleen cells from the non-tg ancestral background mice were similarly stimulated with the same rSAgs mixture.

Each point represents the mean of triplicate $\pm \mathrm{SD}$; shown is a representative of one from at least four independent experiments. 
This was not related to differences in HLA allele expression inasmuch as flow cytometric analysis showed comparable expression of DQ6 and DQ8 (Table 2.1). DR4 expression was lower than that of DQ6 and DQ8 because cDNA was used for the generation of the DR4-mice, and genomic DNA was used to create the DQ6 and DQ8 tg-mice (303, 304, 306).

The enhanced responsiveness of the DR4/DQ8 mice compared to DQ6 and DR15 mice was unrelated to expression of double versus single alleles because as demonstrated in Figure 2.1A, mice expressing DQ8 only were also significantly higher responders to the M1T1 SAgs as compared to DR15 and DQ6 mice.

\section{Dominance of the effect of HLA allelic variation in potentiating M1T1 SAgs responses}

The possibility that TCR V $\beta$ repertoire variability may be a major factor in the stark differences in responses between the various mice was addressed. We found the V $\beta$ repertoire in DQ6 and DR4/DQ8 to be quite comparable with few exceptions (Fig. 2.3). The percentage of T cells expressing TCR V $\beta 5$ was higher in the DQ6 mice while 10 and 14 were higher in the DR4/DQ8 mice but these elements are not specific to any of the SAg produced by the M1T1 strain $(103,307)$. The expression of TCR V $\beta 11$ and 12, which are specific for SmeZ1 was higher in the DQ6 mice, yet these mice were low responders. Thus we conclude that variation in the TCR V $\beta$ repertoire does not account for the enhanced responsiveness of DR4/DQ8 mice to M1T1 SAgs.

To further confirm this, we performed crisscross SAg presentation assays, where pure T cells from the DQ6 or the DR4/DQ8 mice were stimulated with the same 
Table 2.1 Percentages of splenocytes expressing respective HLA class II transgenes and the mean fluorescence intensity (MFI) of the expression ${ }^{\mathrm{a}}$.

\begin{tabular}{lcccc}
\hline HLA tg-mice & $\%$ DQ & $\%$ DR & DQ MFI & DR MFI \\
& & \multicolumn{3}{c}{ \pm SD } \\
\hline HLA-DQ6 & $36 \pm 4.3$ & - & $273 \pm 24.7$ & - \\
HLA-DR4/DQ8 & $37 \pm 2.6$ & $21.9 \pm 1.6$ & $292.4 \pm 30.5$ & $101.2 \pm 6.6$ \\
\hline
\end{tabular}

${ }^{a}$ Analysis of HLA class II expression in tg mice. Spleen cells from 4-5 naïve DR4/DQ8 or DQ6 mice were analyzed by flow cytometry for surface expression of the transgene DR4, DQ8 and DQ6 as described in material and methods. The data are presented as the mean $\pm \mathrm{SD}$. 


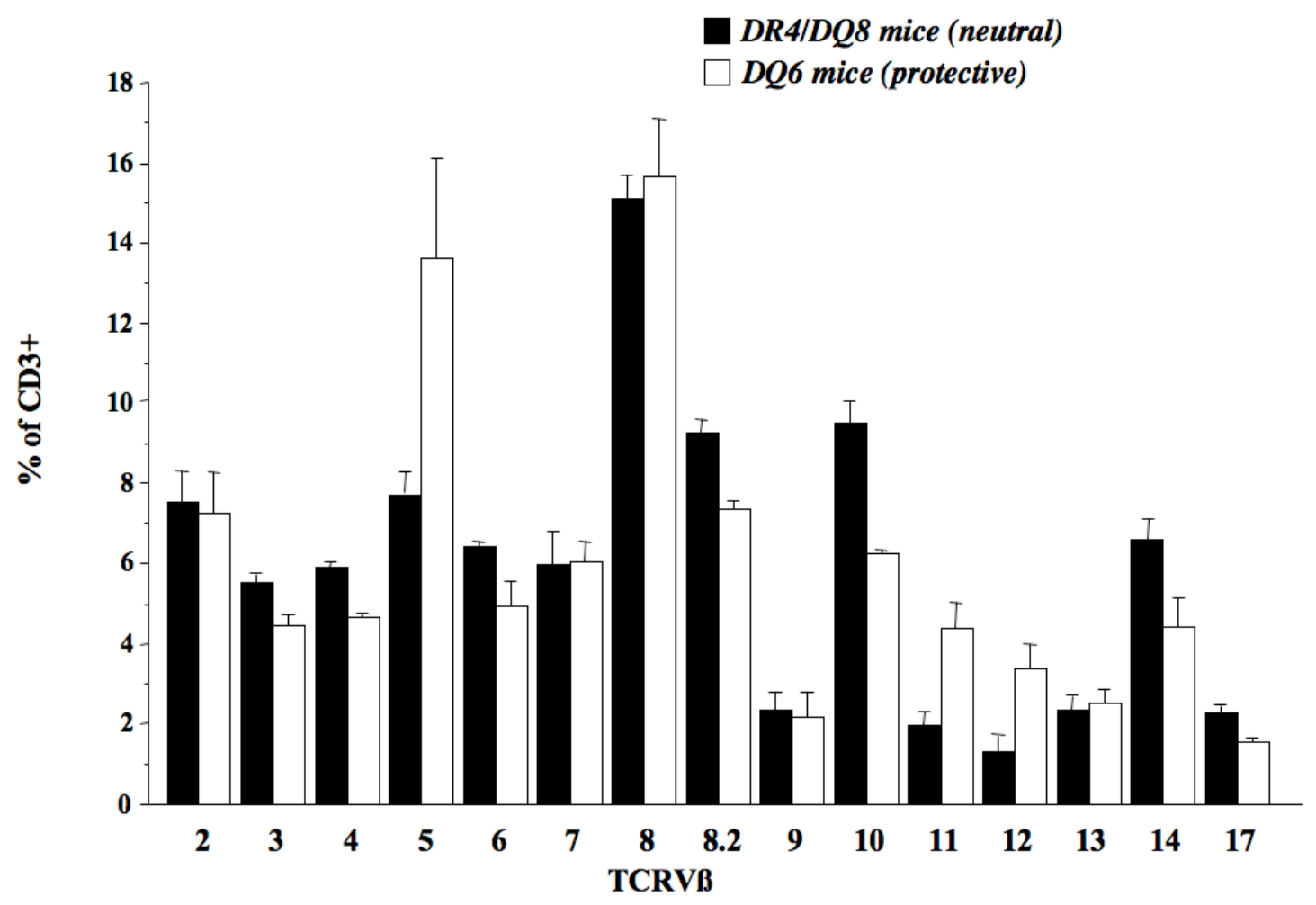

Figure 2.3 Comparison of the TCR V $\beta$ repertoire of the DQ6 and DR4/DQ8 mice.

Splenocytes from age-matched DR4/DQ8 and DQ6 tg mice $(\mathrm{n}=3$ mice $/$ strain $)$ were stained for CD3 and TCR V $\beta$-specific fluorochrome-conjugated antibodies and analyzed by flow cytometry. Each bar represents the mean \pm SD of the percentages of cells expressing a specific TCR V $\beta$ subfamily within the $\mathrm{CD}^{+}$population. 
M1T1 SAgs, presented either by their autologous HLA class II-expressing APC or by APC from the other HLA-tg mouse. The presentation of the M1T1 SAgs by DQ6expressing APC resulted in a two-fold lower proliferative response than the presentation by the DR4/DQ8-expressing APC ( $\mathrm{p}<0.01)$, regardless the source of the responding $\mathrm{T}$ cells (Fig. 2.4A). The dominant effects of the HLA class II allelic variation on the response was also reflected in TNF- $\alpha$, IFN- $\gamma$, and IL-2 responses, where in the presence of the DQ6 APC there was respectively $2(\mathrm{p}<0.01), 13(\mathrm{p}<0.01)$, and 3 -fold $(\mathrm{p}<0.001)$ lower cytokine level compared to cultures containing the DR4/DQ8 APC (Fig. 2.4B). These data demonstrate that T cells from the DQ6 mice are perfectly capable of mounting potent responses to the streptococcal SAgs, if presented by the DR4/DQ8 APC, but that the magnitude of the response is governed mainly by the HLA class II allele type expressed on the presenting APC.

To further confirm the dominant role of class II allelic variation in modulating the SAg response we investigated the effect of different human HLA class II alleles expressed by murine APC on the SAg response of pure human T cells. Similar to the murine $\mathrm{T}$ cells, human $\mathrm{T}$ cells proliferative (Fig. 2.5A) and cytokine (Fig. 2.5B) responses to the same M1T1 SAgs were significantly $(\mathrm{p}<0.01)$ lower in the presence of the DQ6 APC as compared to the DR4/DQ8 APC.

\section{HLA class II allelic variation directly modulates the severity of systemic disease and mouse survival following infection with M1T1 GAS}

We have shown that individuals carrying the protective DR15/DQ6 alleles are significantly more protected against SSD in invasive infection, compared to others 
Figure 2.4 Cross-presentation of the M1T1 SAgs by APC from DQ6 and DR4/DQ8 mice to each other's T cells reveals that variation in HLA class II is the main controller of the magnitude of SAg.

A. Proliferative responses. DQ6 (protective) and DR4/DQ8 (neutral) T cells were separated from their autologous APC. The native mixture of M1T1 SAgs (at 1:500 dilution) was presented to the pure T cells (1X10 /well) from DQ6 or DR4/DQ8 mice by mitomycin C-treated APC $\left(0.5 \times 10^{5} /\right.$ well $)$ from either DQ6 or DR4/DQ8 mice.

$B$. Cytokine response of pure T cells from DQ6 mice to native M1T1 SAgs presented by APC from either DQ6 or DR4/DQ8 mice. The culture supernatants were collected at 24, 48 , and $72 \mathrm{~h}$ culture and analyzed for of IL- $2, \mathrm{TNF}-\alpha$ and IFN- $\gamma$ production. Differences in cytokine levels are in support of the observed proliferative responses. The results shown represent one out of four separate experiments.

$\mathrm{P}<0.05:^{*}, \mathrm{P}<0.01:^{* *}, \mathrm{P}<0.001:^{* * *}$ based on all experimental results using MannWhitney U test. 
A

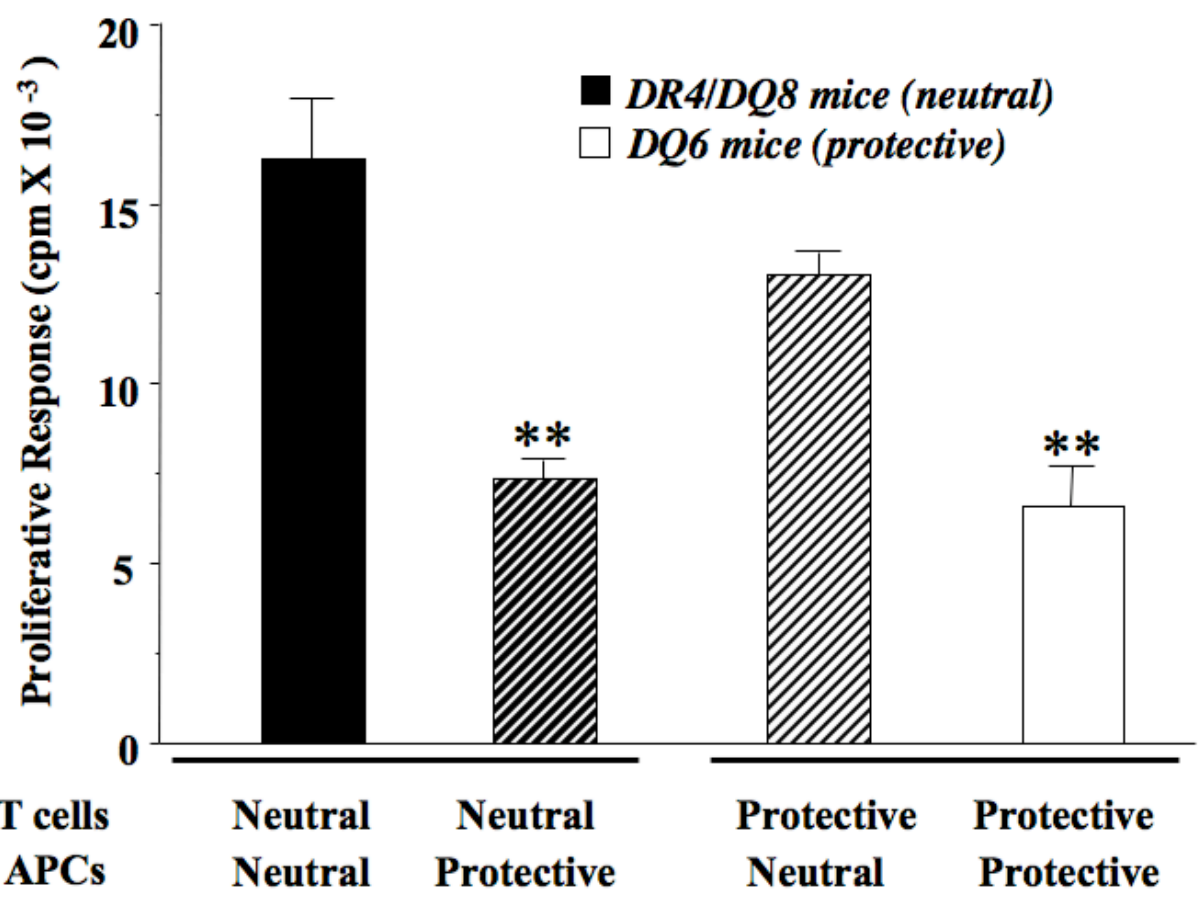

B

$$
\text { DQ6 T Cells }+ \text { APC } \longrightarrow \square D R 4 / D Q 8 \text { mice (neutral) }
$$
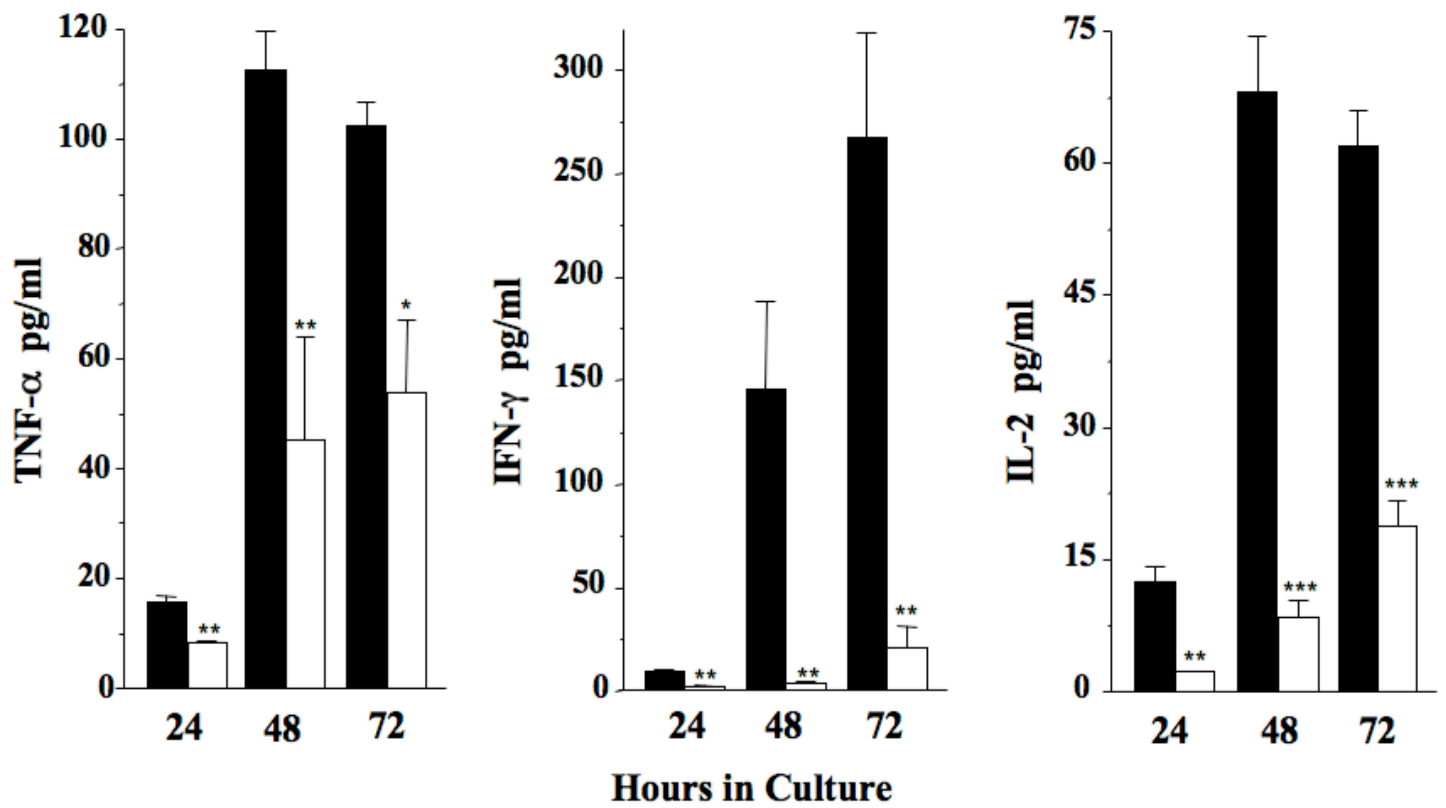
Figure 2.5 Differential proliferative and cytokine responses of APC-depleted human T cells to native M1T1 SAgs presented by APC from DQ6 or DR4/DQ8 mice.

A. Pure human T cells (1X10 /well) were stimulated with 1:500 dilution of M1T1 SAgs in the context of mitomycin C-treated APC $\left(0.5 \times 10^{5} /\right.$ well $)$ from DQ6 or DR4/DQ8.

$B$. Human T cells were stimulated in the same way as above and culture supernatants were collected at 24, 48, and 72h culture, and analyzed for IL-2, TNF- $\alpha$ and IFN- $\gamma$

expression using the human. Each data set represents mean \pm SD of triplicates. Shown are the results representing one of three independent experiments. $\mathrm{P}<0.05: *, \mathrm{P}<0.01$ : $^{* *}$, $\mathrm{P}<0.001: * * *$ based on results from all experiments using Mann-Whitney $\mathrm{U}$ test. 


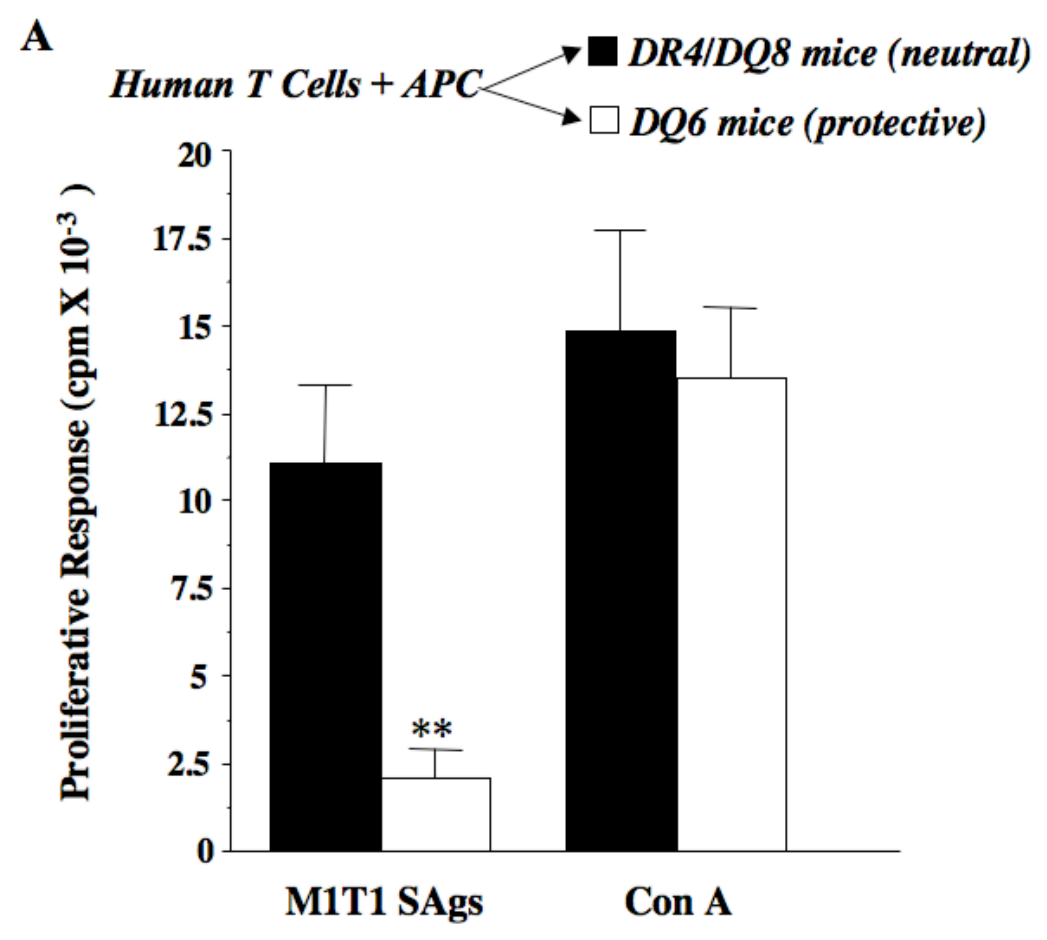

B
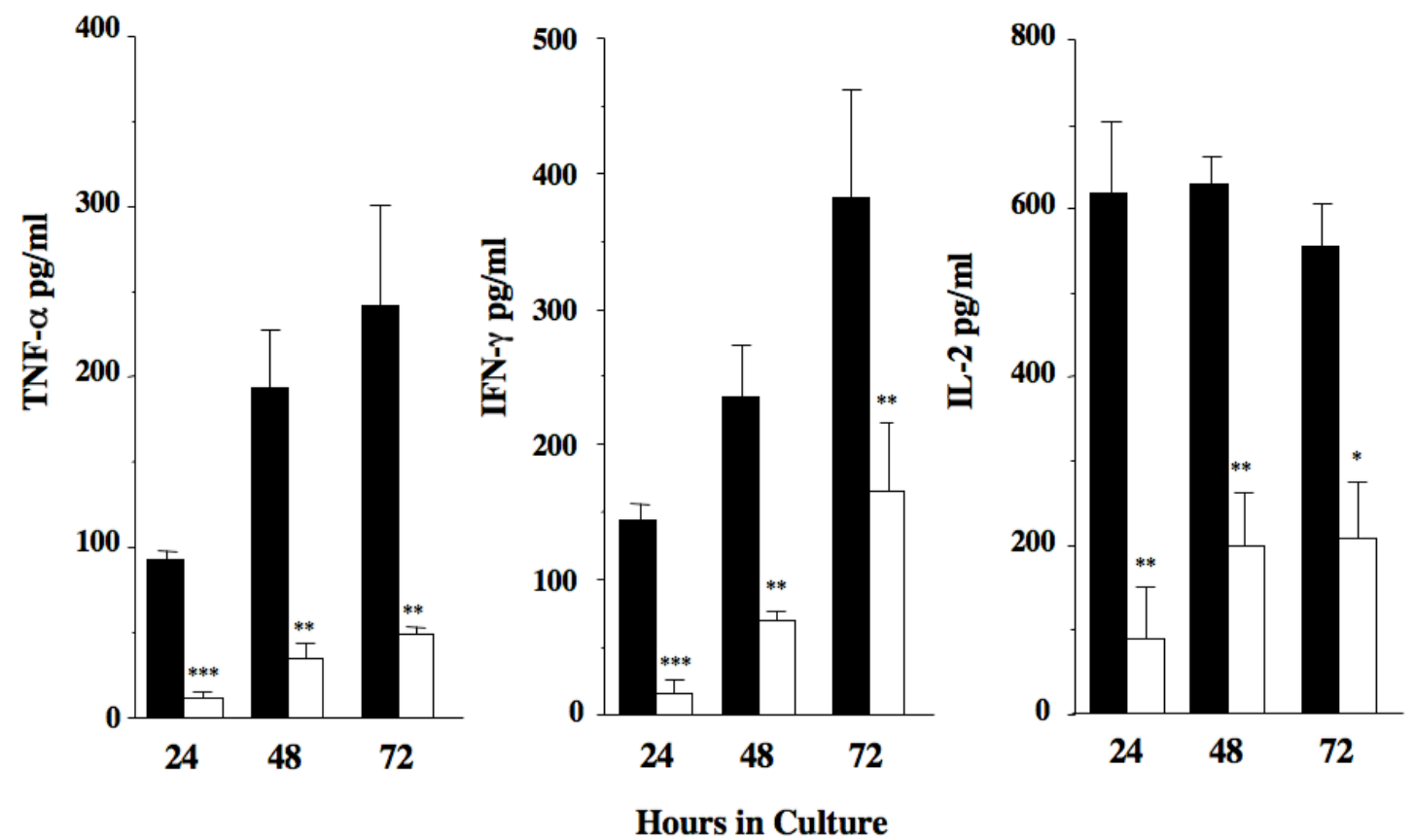
infected with the same GAS strain, but who are not carrying those alleles (275).

Therefore, it was important to compare in vivo responses of DQ6 and DR4/DQ8 mice and see if they are comparable to what we found in humans and whether the significant differences observed in our in vitro studies would translate to considerable biologic variation in vivo. Accordingly, we infected the HLA tg-mice i.v., with 5X10 $0^{6} \mathrm{CFU}$ of the M1T1 bacteria, and recorded their mortality over a period of 7 days. DR4/DQ8 mice started to succumb to the infection within $48 \mathrm{~h}$ with a mortality rate of $80 \%$ by day 4 after inoculation. By contrast none of the DQ6 mice died during the observation period (Fig. 2.6A).

The differential susceptibility of the HLA tg-mice to infection with M1T1 bacteria was also reflected in the systemic levels of the inflammatory cytokines TNF- $\alpha$ and IFN- $\gamma$. Plasma levels of TNF- $\alpha$ and IFN- $\gamma$ at 24 h post i.v., infection with M1T1 bacteria were significantly lower in the DQ6 mice than in the DR4/DQ8 mice $(\mathrm{p}<0.01$ and $\mathrm{p}<0.05$, respectively) (Fig. 2.6B). In contrast to the significant difference in the survival and systemic inflammatory cytokines results, both mice strains had comparable bacteremia at $24 \mathrm{~h}$ post infection as well as comparable bacterial load in the liver at 24 and 48h post infection (Fig. 2.6C). However, DR4/DQ8 spleens showed significantly higher bacterial load than those of DQ6 only at 24 h post infection $(\mathrm{P}=0.03)$; but there was no significant difference at the $48 \mathrm{~h}$ in both strains. These observations underscore the role of HLA class II as a major controller of the inflammatory cytokine response and hence the severity of invasive GAS infection. 
$\mathbf{A}$

DR4/DQ8 mice (neutral) $(n=10)$

$\triangle D Q 6$ mice (protective) $(n=10)$
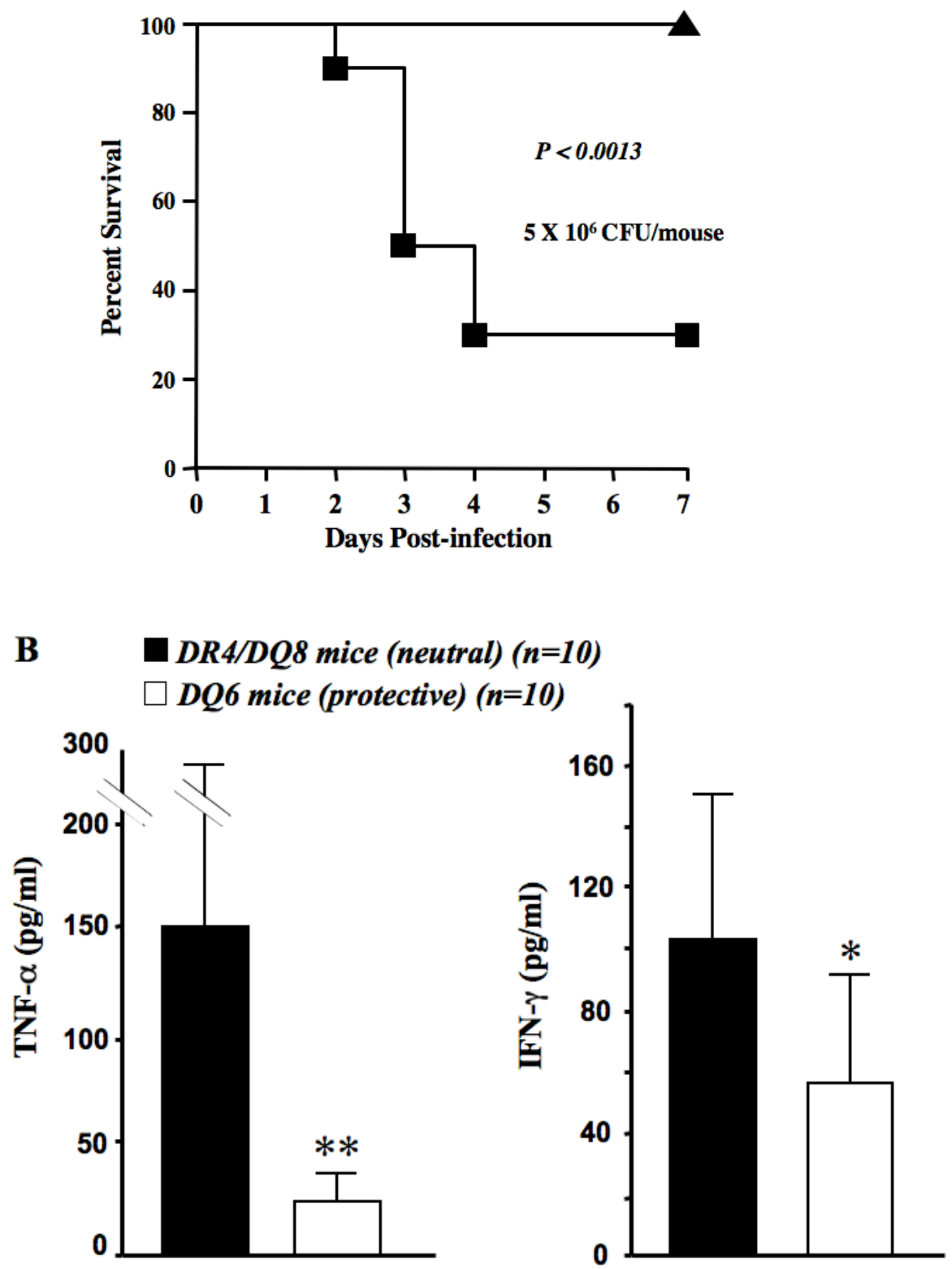

Figure 2.6 Class II allelic variation directly modulates systemic disease severity and survival following infection with M1T1 GAS.

A. In vivo differential susceptibility of HLA-tg mice to M1T1 GAS sepsis. (5X10 6 CFU) live M1T1 bacteria in 250 ul PBS were injected i.v. into DQ6 (A) or DR4/DQ8 (ם) mice. Mice survival was monitored twice daily and death was recorded over 7 days. $B$. IFN- $\gamma$ and TNF- $\alpha$ levels in plasma of HLA-tg mice, $24 \mathrm{~h}$ post infection. $C$. Bacterial load in the blood, liver, and spleen of DQ6 $(\boldsymbol{\Delta})$ or DR4/DQ8 ( $\mathbf{\square})$ mice i.v. infected with $5 \mathrm{X} 10^{6}$ CFU bacteria . $\mathrm{P}<0.05$ : $^{*} \mathrm{P}<0.01$ :** $^{* *}$ 


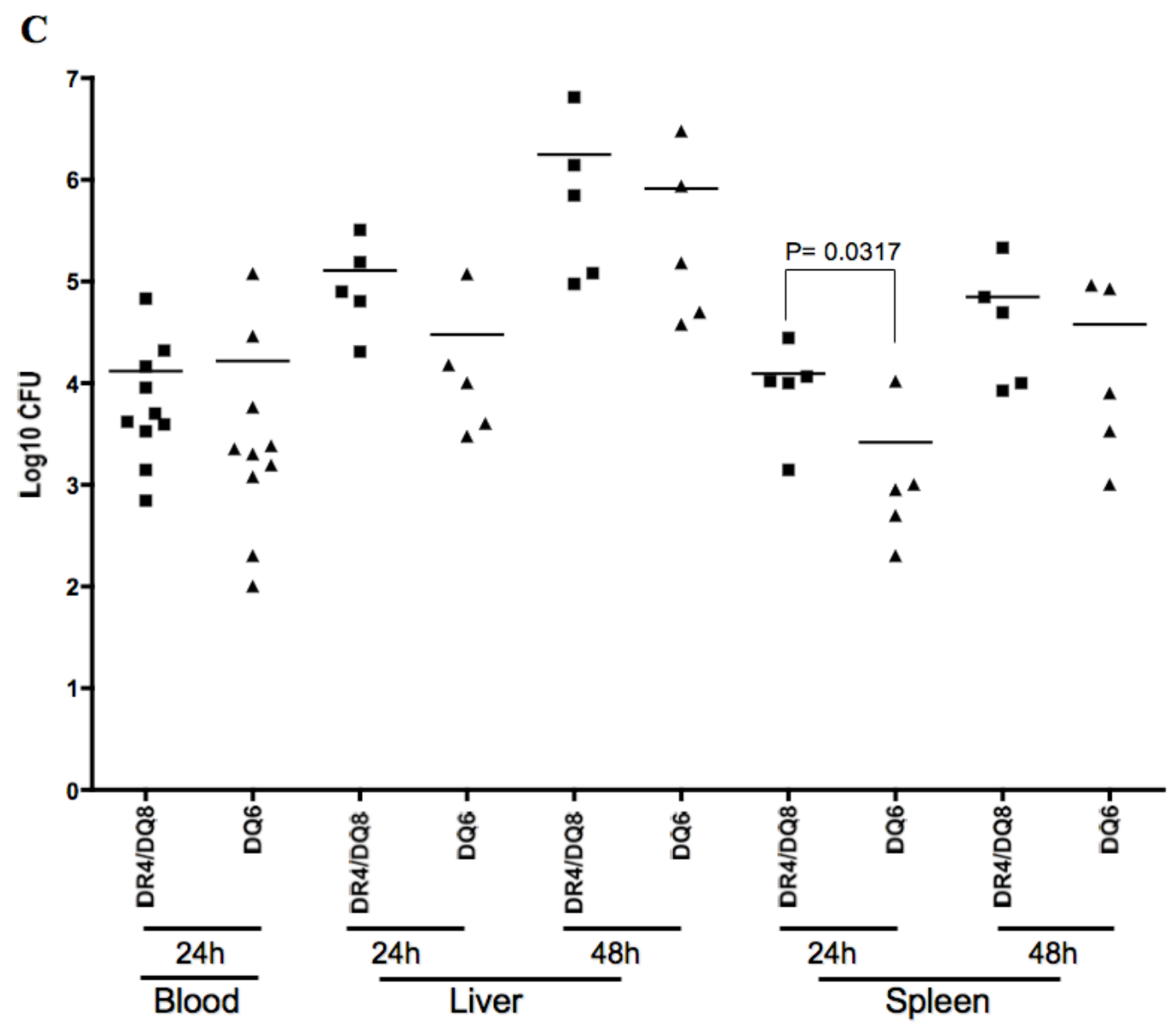

Figure 2.6 (continued). 


\section{Discussion}

The resurgence of invasive GAS infection pandemic in the 1980's, with aggressive manifestations such as STSS and NF, has been receiving much attention for the past 25 years $(221,292,308,309)$. Throughout this pandemic, the clonal M1T1 strain was the most frequently isolated strain from severe and non-severe invasive cases $(25$, $310,311)$. Among the many virulence factors produced by the bacteria, the streptococcal SAgs are mainly responsible for triggering potent inflammatory responses, which mediate the systemic effects seen in patients with STSS $(100,214,312)$. Our previous work provided evidence that certain individuals are prone to be high responders to GAS SAgs, whereas others mount moderate or low inflammatory responses $(275,276)$. We also found that among patients who recovered from invasive GAS infections, those who are prone to be high responders to GAS SAgs in vitro were the ones who had developed severe invasive systemic disease and STSS and mounted very high inflammatory cytokine response during the acute infection; whereas, those who developed mild invasive disease were consistently low responders. Differences between the high and low responders reflected host genetic variations that were found to be associated with the HLA class II locus. Specifically, the strongest association was with DRB1*15/DQB1*06 haplotype, which seemed to confer strong protection from the severe manifestations of the invasive disease (275). However, there was still a question of whether the observed association is directly related to HLA class II allelic variation or to other genes that are in linkage disequilibrium with these HLA haplotypes. In this study we provide evidence for direct role of HLA class II allelic variation in modulating the severity of GAS sepsis, and demonstrate the dominant effect of the protective alleles in attenuating SAgs responses. 
Although well-designed epidemiological association studies have an advantage of being carried out in patients with clear clinical outcomes, these studies are limited by the inability to design certain controlled experimentation to delineate disease pathogenesis. Despite limitations, animal models for diseases remain indispensable to address such drawbacks. Regular mice are generally not a model of choice for STSS because they respond poorly to SAgs, particularly to the streptococcal SAgs $(99,296,297)$. To elicit appropriate responses in these mice, investigators have resorted to using doses of SAgs that are much higher than physiologic $(124,313)$, have augmented SAg effects by coadministering LPS $(296,314)$, or treated the mice with D-galactosamine to prolong the biologic half-life of cytokines by delaying their hepatic clearance (99). Whereas these models provided valuable information, these manipulations may not represent actual events of the human disease.

By contrast, the humanized HLA class II-tg mice have shown high sensitivity to bacterial SAgs; T cells from these mice respond vigorously to bacterial SAgs in a way similar to human $\mathrm{T}$ cells without any manipulation, suggesting a SAg presentation pattern comparable to that in humans $(227,300)$. Several studies have used HLA-tg mice to investigate immune responses to SAgs and SAg-producing bacteria $(227,297,300,301)$. We were particularly interested in HLA-tg mice carrying the HLA class II alleles associated with protection or high-risk for SSD, as identified in our epidemiological study (275). However, as mentioned earlier, mice carrying the high-risk alleles are not yet available so we compared responses to M1T1 SAgs of mice carrying the neutral alleles DR4 and/or DQ8 alleles, to mice carrying the protective DQ6 allele.

Our previous studies showed that PBMCs isolated from healthy individuals or 
recovered patients expressing HLA alleles associated with protection from STSS mounted significantly lower proliferative and cytokine responses to SAgs than PBMCs from individuals carrying neutral or high-risk alleles (275). In a nicely reproducible manner, the in vitro responses of HLA tg-mice splenocytes to M1T1 SAgs were markedly lower for the DQ6 mice than for the DR4/DQ8 mice. Also, in human invasive GAS infections, we showed that patients with SSD who lack the DR15/DQ6 protective alleles mount significantly higher levels of inflammatory cytokine responses, in both acute and convalescent phase of the disease, than those with mild non-severe disease $(275,276)$. We found the same pattern in the HLA tg-mice, where splenocytes from the DQ6 mice mounted significantly lower levels of the inflammatory cytokines TNF- $\alpha$ and IFN- $\gamma$ in vitro and in vivo than those of the DR4/DQ8 mice. In addition, the DQ6 mice were highly resistant to GAS sepsis, while the DR4/DQ8 mice were susceptible and quite overwhelmed by the infection despite having comparable bacterial load in liver and spleen. These findings emphasize the overriding role of HLA class II in governing the outcome of invasive GAS infection by modulating the systemic inflammatory cytokine responses to SAgs. Additionally, the SAgs-crisscross presentation assays where APC from one HLA-tg mouse were used to present the SAgs to T cells from the other mouse or to human $\mathrm{T}$ cells, showed reproducibly and significantly lower proliferative and cytokine responses in the context of DQ6 APC as compared to the DR4/DQ8 APC. Thus, results from the HLA-tg mice paralleled our findings in humans and confirmed a direct and dominant role of HLA class II allelic variation in controlling the magnitude of response to streptococcal SAgs, with little or no impact of variability in host T cells on disease severity. The dominance of the protective effect of the DQ6 allele seen in 
humans was also confirmed in these HLA-tg mice.

Differences in proliferative and inflammatory cytokine responses to the M1T1 SAgs when presented by DR4/DQ8 as compared to DQ6 or DR15 alleles may be attributed to difference in binding affinities or to the ensuing cellular signals in the APC and T cells. Our previous studies (288) indicated no correlation between binding affinity and response level. However, ongoing studies in our lab are addressing this possibility in greater depth as well as examining if differences in the stability of the trimolecular complex (composed of Class II-SAg-TCR) taken as a whole may establish the overall strength of the response. Although our present study point to the HLA class II molecule as the principal controller of the SAg-induced response, we still cannot rule out the likelihood that associated polymorphism in other host genes could contribute to susceptibility to STSS

These findings should facilitate our ongoing studies of the temporal events that take place in the pathogenesis of invasive GAS infection and will help us identify specific molecular and cellular mechanisms involved in regulating SAg responses in the presence of distinct HLA class II alleles in vivo. 


\title{
Chapter 3. HLA Allelic Variants Dictate the Overall Cytokine Profile Elicited by Superantigens: Streptococcal Toxic Shock Protective Alleles Elicit IL-10 Production and Diminish Inflammatory Responses
}

\begin{abstract}
Introduction
Host genetic variability can profoundly affect resistance/susceptibility to infectious diseases as well as control the severity of disease associated clinical manifestations $(244,246,315)$. Group A Streptococci (GAS) are important human pathogens that can cause a wide spectrum of diseases ranging from superficial infections to life threatening streptococcal toxic shock syndrome (STSS) and necrotizing fasciitis (NF). Furthermore, in genetically susceptible individuals, GAS infections can lead to post-streptococcal autoimmune diseases, including acute rheumatic fever, glomerulonephritis, carditis, arthritis or neurological disorders (10, 293, 316). The severe forms of invasive GAS infections, including STSS and NF resurged in the 1980s after almost 60 years of decline $(221,292)$. Coincidental with the resurgence of severe invasive GAS disease was the emergence of a particular subclone of the M1T1 serotype, which exhibited increased virulence, global dissemination and persistence for over 25 years (22). The increased virulence of this M1T1 clone is in part attributed to the acquisition of 3 unique prophages, one carries the potent SAg SpeA2, and the other two carry DNases, including a novel and highly potent streptodornase (Sda1) (6). Despite its increased virulence, this M1T1 strain was isolated from individuals with starkly different clinical manifestations, thereby underscoring the critical role of host factors in modulating outcomes of GAS infections (273-275).
\end{abstract}


Although GAS possess a large battery of virulence factors that can trigger potent inflammatory responses in the host $(9,317,318)$, the superantigenic streptococcal pyrogenic exotoxins (Spes) are the main players in severe invasive GAS infections, including GAS sepsis and STSS $(100,275)$. SAgs bridge HLA class II molecules on antigen presenting cells (APC) and the TCR on T cells, thereby forcing these cells to interact regardless of the antigenic specificity of the TCR. In addition, SAgs utilize these receptors to trigger excessive activation of both cell types, causing massive $\mathrm{T}$ cell proliferation and inflammatory cytokines production. However, several studies have shown that not all class II isotypes or alleles present a given SAg equally - some SAgs are presented better by DR than by DQ and vice versa $(285,287-289)$.

The magnitude of inflammatory responses triggered by the same Strep SAg in different individuals can also vary considerably (206), and there is a direct correlation between the levels of inflammatory cytokine responses and severity of invasive disease $(275,276)$. These observations suggested that variations in host immunogenetic factors involved in regulating SAg responses are important determinants of infection severity. Indeed, we identified significant associations between specific HLA haplotypes and different manifestations of GAS sepsis. Specifically, the HLA DRB1*1401/DQB1*0503 (DR14/DQ5) and DRB1*0701/DQB1*0202 haplotypes were associated with the severe forms of GAS sepsis, namely STSS, whereas the HLA DRB1*1501/DQB1*06 (DR15/DQ6) haplotype conferred strong protection from STSS even in septic patients (275). Biological validation studies in vitro and in vivo using animal models of GAS sepsis demonstrated that disease-modulatory effects are directly mediated by HLA allelic variation and not by other genes in linkage disequilibrium on the same haplotype (275, 
319). However, SAg responses are also influenced by the interaction with its other receptor on T cells. SAgs interact with specific TCR V $\beta$ elements of the $\beta$ chain of the $\alpha \beta$ TCR with little contribution from the other variable elements of the TCR $(313,320$, 321). Humans have about 25 major V $\beta$ families (322). Each SAg has a distinct, signature TCR V $\beta$ specificity and can interact with $T$ cells expressing those $V \beta$ elements regardless of the Ag specificity of their TCR. Therefore, the response to particular SAg could be affected by the relative abundance of SAg-reactive TCR V $\beta$ families and the frequency of T cells expressing those families in a given individual's repertoire.

In this study we determined the relative contribution of variations in the individual's HLA class II and TCR V $\beta$ repertoire on host responses to Strep SAgs derived from the prevalent M1T1 strain and we investigated the molecular basis for the protective effect of the DR15/DQ6 against STSS. We found a dominant role of HLA class II variability in controlling the magnitude of Strep SAgs responses, with little to no effect of TCR V $\beta$ variation on the overall SAg response. Additionally, we show that alleles associated with protection from STSS seem to diminish proliferative and inflammatory cytokine responses to the Strep SAgs by inducing the production of high levels of the regulatory cytokine, IL-10.

\section{Materials and Methods}

\section{Preparation of partially purified Strep SAgs from GAS culture supernatants}

Bacterial culture supernatants were prepared from overnight cultures of GAS isolates by ethanol precipitation followed by extensive dialyses, as detailed 
elsewhere(319). We have previously shown that these partially purified culture supernatants are good sources of the SAgs mixture produced by the clinical isolates (274, 319).

\section{Cells}

We isolated peripheral blood mononuclear cells (PBMC) from various individuals as previously described (323). T cells were purified by subjecting freshly isolated PBMC to one cycle of erythrocyte resetting, followed by using the human pan $\mathrm{T}$ cell isolation kit and autoMACS according to the manufacturer's instructions (Miltenyi Biotec, Auburn, CA). To remove any residual APC, we incubated the purified cells overnight at $37^{\circ} \mathrm{C}$ in RPMI 1640 supplemented with 5\% (v/v) FBS, 4 mM L-glutamine, 25 mM Hepes, 50 $\mathrm{U} / \mathrm{ml}$ penicillin/ 50 $\mu \mathrm{g} / \mathrm{ml}$ streptomycin (RPMI complete medium). In each experiment, the purity of the APC-depleted T cells was confirmed phenotypically by flow cytometry and functionally by the lack of response to PHA or the M1T1 SAgs. Experiments in which purified T cells (cultured alone without any APC) showed a response to PHA or SAgs were discarded.

The E-rosette-negative fraction, enriched in B cells and monocytes, were used as APC in cross presentation assay, and their purity (T cell depletion) was determined in each experiment by their non-responsiveness to PHA or the M1T1 SAgs.

B cell lines homozygous for the high risk DRB1*1401/DQB1*0503 or the protective DRB1*1501/DQB1*0602 haplotypes were obtained from the European Cell Culture Collection (UK). To use these cells in presenting SAgs, we treated them with mitomycin C (25 ug/1X10 cell/ml, Sigma) for $30 \mathrm{~min}$ at $37^{\circ} \mathrm{C}$ followed by extensive 
washing with Hank's balanced salt solution. The efficacy of mitomycin C treatment was checked by $\left[{ }^{3} \mathrm{H}\right]$ thymidine uptake and compared to untreated cells.

\section{T cell proliferation assay}

PBMC $\left(2 X 10^{5} /\right.$ well $)$ or pure T cells $\left(10^{5} /\right.$ well) were cultured in RPMI complete medium in 96 well plate. T cells were cultured with the indicated numbers of autologous or allogeneic APCs, or with mitomycin C-treated B cell lines $\left(0.5 \times 10^{5} /\right.$ well $)$. Cells were stimulated with either PHA $(1 \mu \mathrm{g} / \mathrm{ml})$ or several dilutions of the mixture of native secreted M1T1 SAgs. The amount of M1T1 SAgs used was predetermined in titration assays to select SAg dilutions within the linear proliferation curve and not the plateau area. This is important to allow detection of differences in responses due to either HLA or TCR variations. In some experiments exogenous recombinant human IL-10 (3ng/ml)(R \&D systems) was added to individual "B" PBMC or to T cells co-cultured with B cell line homozygous for the high-risk haplotype. After incubation for $72 \mathrm{~h}$ at $37^{\circ} \mathrm{C}$ in a $5 \% \mathrm{CO} 2$ and $95 \%$ humidity, the cultures were pulsed for the last $6 \mathrm{~h}$ with $1 \mu \mathrm{Ci} /$ well $\left[{ }^{3} \mathrm{H}\right]$ thymidine (specific activity $=6.7 \mathrm{Ci} / \mathrm{mmol}$; Dupont, Wilmington, DE). All samples measurements were performed in triplicates, and the data were presented as mean cpm $\left[{ }^{3} \mathrm{H}\right]$ thymidine uptake \pm SD. Each experiment was repeated at least three times.

\section{Cytokine assays}

Culture media were collected from the individual wells at 24,48 , and $72 \mathrm{~h}$ poststimulation, cleared of cells by centrifugation, and stored at $-80{ }^{\circ} \mathrm{C}$ for subsequent analysis. The cytokines IL-2, IL-4, IL-6, IL-10, IFN- $\gamma$, TNF- $\alpha$ were simultaneously 
quantified, using the human cytokine LINCOplex kit (Linco Research Inc., St. Charles, MO). TNF- $\beta$ was analyzed by ELISA kit from R\&D Systems (Minneapolis, MN). The assays were performed according to the manufacturer's instructions, and the concentrations were calculated from standard curves run in parallel with the test samples. All samples measurements were performed in triplicates and data are presented as mean concentration $\pm \mathrm{SD}$.

\section{Analysis of the $T$ cell receptor $\mathrm{V} \beta(\mathrm{TCR} \mathrm{V} \beta)$ repertoire by flow cytometry}

Quantitative analysis of TCR V $\beta$ repertoire was conducted by flow cytometry using the IOTest Beta Mark TCR V $\beta$ repertoire Kit (Beckman Coulter, cat \# IM3497). CD3-PC5 conjugate was used to enable proper gating on T cells only. Approximately $5 \mathrm{X} 10^{5}$ lymphocyte/tube in 100ul PBS containing $1 \%$ BSA were stained with mAbs for different $\mathrm{V} \beta$ according to the manufacturer's instructions. Using a FACSCalibur flow cytometer (Becton Dickinson), we gated on the CD3-PC5 cells and analyzed three different TCR V $\beta$ specificities per tube. The data were analyzed using FlowJo software (Tree Star, Ashland, OR 97520). A minimum of 30,000 cell events was acquired for each analysis.

\section{Flow cytometric assessment of HLA DQ and DR expression level}

We used monoclonal antibody (mAb) labeled with phycoerythrin (PE) (Catalog. No. 347367, BD) specific to HLA-DR and primary unlabeled mAb specific to HLA-DQ (MCA379G, serotec) followed by PE-labeled secondary Ab (STAR12A, serotec). Analysis was conducted on a FACSCalibur (Becton Dickinson). 


\section{Statistical analysis}

Analysis of variance (ANOVA) or a two-tailed Student's $t$ test was used to determine the significance of the data. A $p$ value of $<0.05$ was considered significant.

\section{Results}

\section{HLA Class II allelic variation plays a dominant role in determining the magnitude and type of Strep SAg responses}

In initial studies, we selected two individuals, one individual "A" expresses the DR15 and DQ6 alleles associated with strong protection from STSS and the other, individual "B" expresses the DR7 and DQ5 alleles associated with high risk for it, and analyzed the relative contribution of HLA class II allelic variation to responses elicited by Strep SAgs produced by the prevalent M1T1 GAS strain. Although we had shown that the same strain causes disease of different severity in genetically distinct individuals, we decided to test the response of both individuals to two invasive M1T1 GAS isolates: isolate 1 was recovered from an STSS case and isolate 2 was from a non-severe invasive case. We compared proliferative responses of PBMC from individuals "A" and "B" to the SAgs produced by these isolates, and found that both isolates elicited the same level of response in both individuals; however, there were significant differences in the level of response between these individuals. Individual "A" reproducibly mounted significantly $(p<0.01)$ lower proliferative responses to the M1T1 SAgs than individual "B" (Fig. 3.1). This difference was SAg specific because both individuals had comparable PHA responses. Furthermore, differences in response levels of both individuals were not due to 


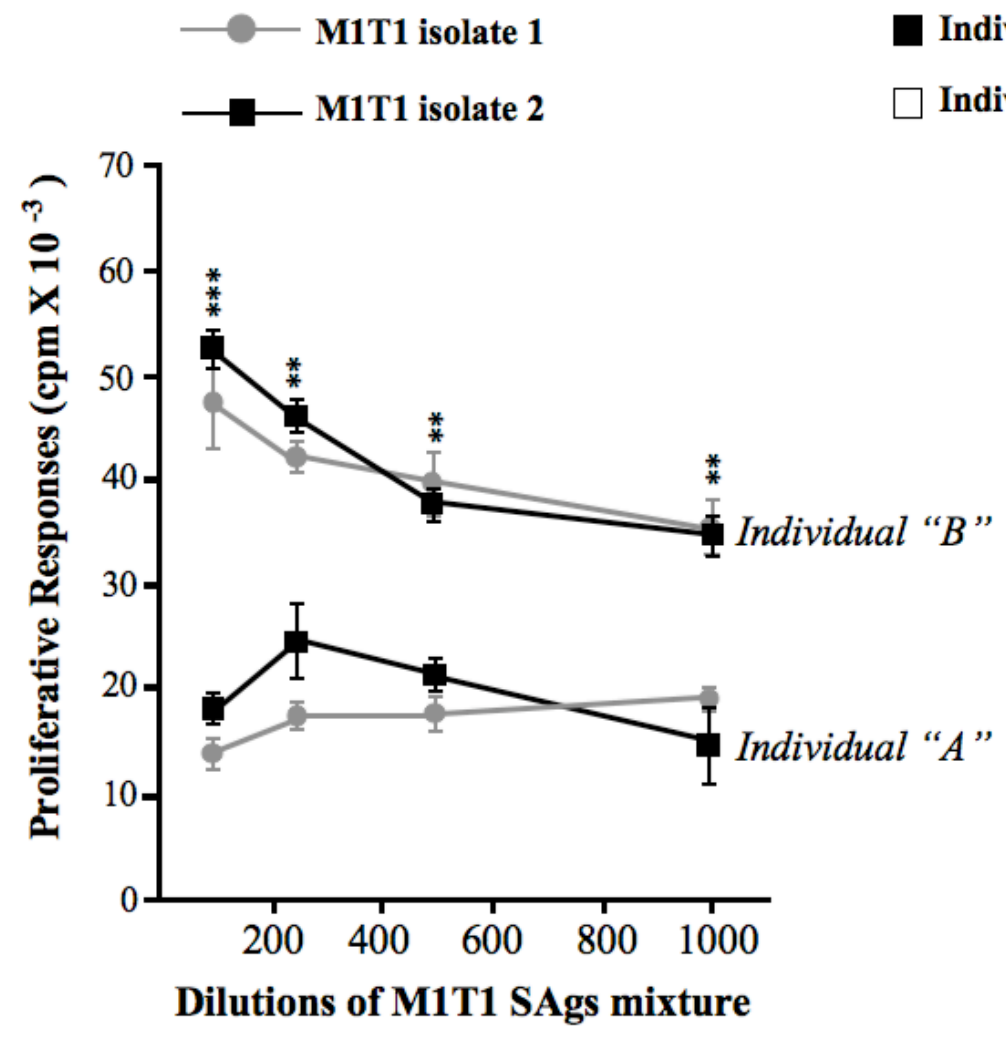

Individual "A": protective haplotype

Individual "B": risk haplotype

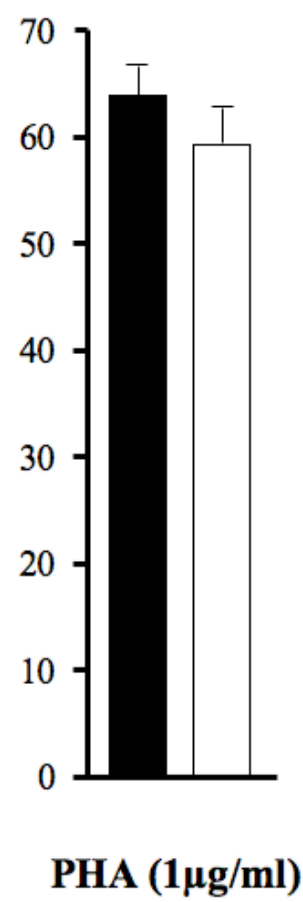

Figure 3.1 Proliferative responses of PBMC from individual "A" (protective haplotype) and "B" (risk haplotype) to the native mixture of partially purified M1T1 SAgs.

PBMC (2X10 $/$ well) from individual "A" (expresses STSS protective HLA class II alleles) and individual "B" (expresses STSS high-risk HLA class II alleles) were stimulated in triplicates with serial dilutions (1:100, 250, 500 and1000) of the SAgs mixture produced by isolate 1 from a severe invasive case, isolate 2 from a non-severe invasive case, or by PHA (1 $\mathrm{ug} / \mathrm{ml})$. The experiments were repeated at least three times, and the presented data are means \pm SD. Statistical significance was assessed by ANOVA $(* * * \mathrm{P}<0.001, * * \mathrm{P}<0.01, * \mathrm{p}<0.05)$. 
variable expression level of DQ or DR because both individuals expressed comparable levels of these antigens as determined by flow cytometry (Table 3.1).

To determine the relative contribution of T cells versus APC in controlling SAg response levels, we performed experiments where we separated the T cells and APC from PBMC of each individual and tested their response to the M1T1 SAgs in cross presentation studies. The presentation of the Strep SAgs to pure T cells from the high responder, individual "B" by APC from individual "A" who carries the protective DR15/DQ6 alleles resulted in a significantly reduced proliferative response compared to that in the presence of his own APC (Fig. 3.2A). Likewise the responses of T cells from the low responder (individual " $A$ ") were significantly increased when the SAgs were presented by APC from the high responder, expressing the high-risk alleles (Fig. 3.2A).

To rule out the possibility that there is something peculiar about the APC and/or T cells from individual "A" or "B", we compared the response of pure T cells from both individuals to the M1T1 SAgs presented by two B cell lines, one homozygous for the STSS protective DRB1*1501/DQB1*0602 haplotype and the other for the high risk DRB1*1401/DQB1*0503 haplotype. As predicted, the presentation by cells expressing the protective DR15/DQ6 haplotype elicited significantly lower proliferative and cytokine responses to the M1T1 SAgs than by cells expressing the DR14/DQ5 haplotype (Fig. 3.2B). The data shown in Figure 2B indicate that when T cells from individual "A" are presented with the M1T1 SAgs in the context of the high risk alleles, they mounted a response that is as potent or even more potent than that of individual " $\mathrm{B}$ ". The response of $\mathrm{T}$ cells from both individuals was diminished in the presence of the DR15/DQ6 expressing cell line (Fig. 3.2B). 
Table 3.1 Percentages of PBMC expressing HLA DR or DQ and the mean fluorescence intensity (MFI) of the expression ${ }^{\text {a }}$.

\begin{tabular}{ccccc}
\hline Individual & $\%$ DQ & $\%$ DR & DQ MFI & DR MFI \\
\hline A & $15.7 \pm 3.4$ & $18.3 \pm 2.3$ & $356.9 \pm 23.4$ & $962.6 \pm 45.7$ \\
B & $16.6 \pm 3.7$ & $19.5 \pm 3.7$ & $334.2 \pm 30.2$ & $1059.2 \pm 34.3$ \\
\hline
\end{tabular}

${ }^{a}$ PBMC from Individual A (with protective alleles) or B (with high-risk alleles) were analyzed by flow cytometry for expression level of DR and DQ. Analysis was performed at each experiment and the data are shown as mean \pm SD. 
Figure 3.2 T cell responses to the M1T1 SAgs are governed by the type of HLA class II alleles expressed by autologous or allogeneic APC expressing STSS protective or highrisk HLA class II haplotypes.

Pure T cells (1X10\%/well) from individuals "A" (protective haplotype) or "B" (high risk haplotype) were stimulated with PHA $(1 \mu \mathrm{g} / \mathrm{ml})$ or an optimal dilution (1:500) of the native mixture of M1T1 SAgs presented by: $A$. Autologous or each other's allogeneic APC $\left(0.5 \times 10^{5} /\right.$ well $)$ in a criss-cross design, or $B$. Immortalized B lymphoblastoid cell lines homozygous for either the STSS protective or high-risk haplotypes as indicated. Proliferative responses were determined as described in Figure 1. The data represent mean cpm \pm SD of at least three independent experiments after normalization for any variability in HLA expression level on the APC. Statistical significance was assessed by ANOVA $(* * * \mathrm{P}<0.001, * * \mathrm{P}<0.01, * \mathrm{p}<0.05)$. 
$\mathbf{A}$

A (individual with protective haplotype)

B (individual with high risk haplotype)
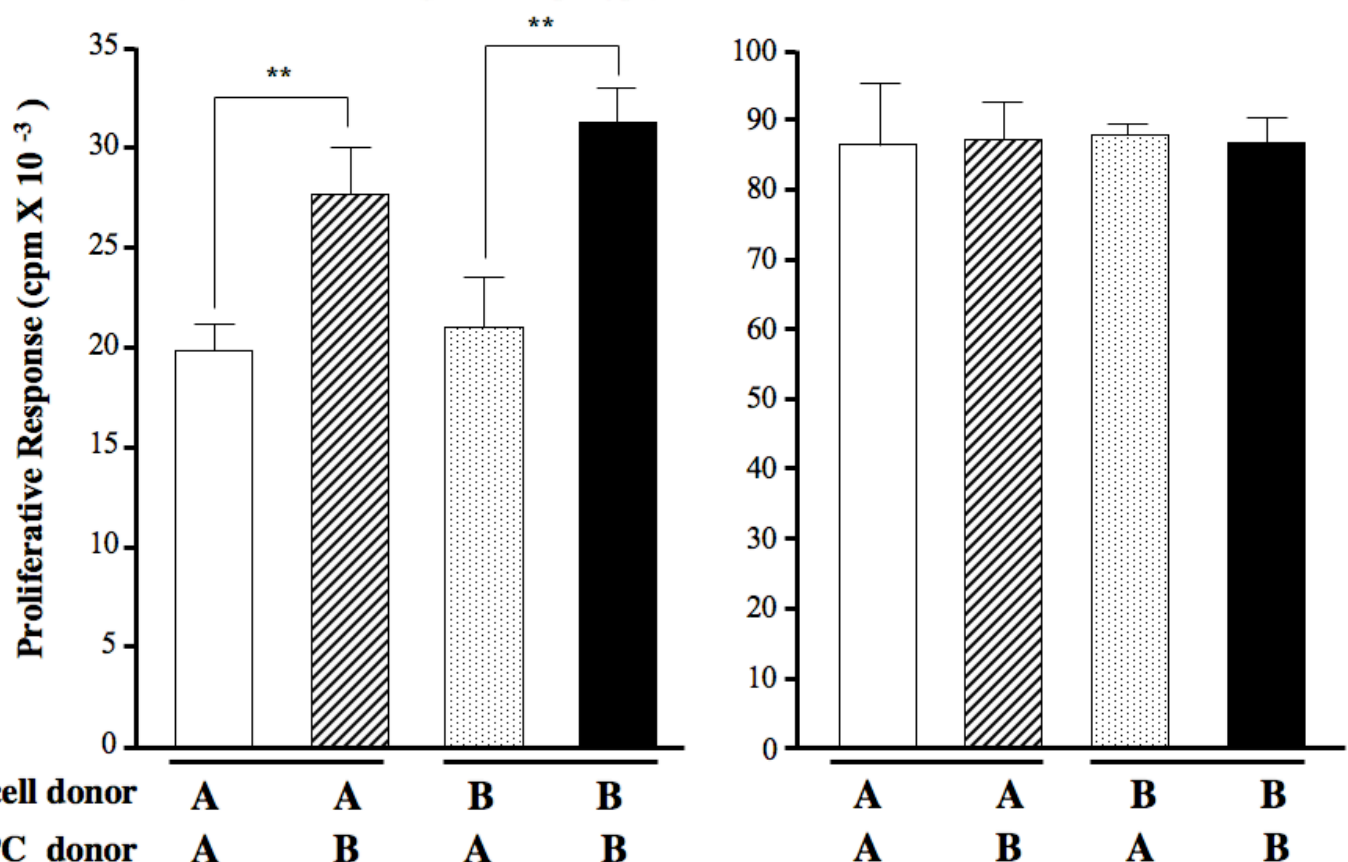

Strep SAgs mixture

PHA $(1 \mu \mathrm{g} / \mathrm{ml})$

B

—-DRB1*15/DQB1*0602 APC (protective)

—— DRB1*14/DQB1*0503 APC (risk)
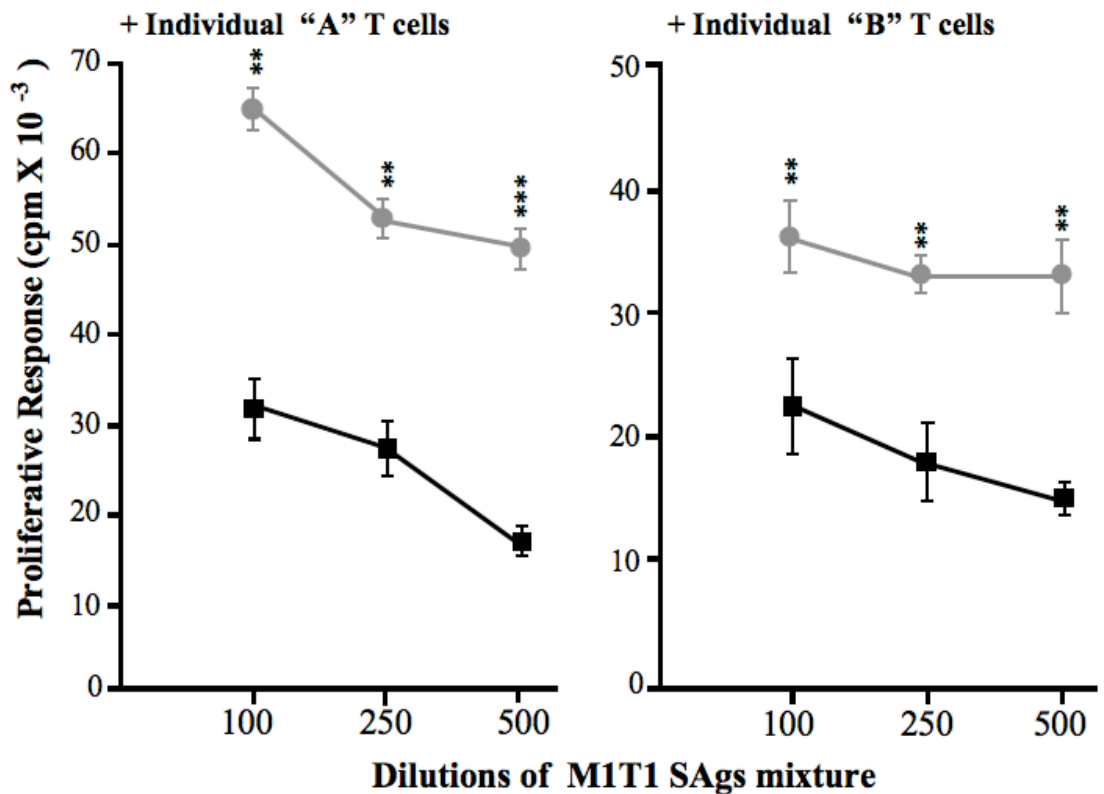
The above data demonstrate that SAg responses are governed primarily by the type of HLA class II alleles expressed on the APC with minimal or no contribution from TCR V $\beta$ repertoire variability. To investigate this possibility further, we compared the TCR V $\beta$ repertoire of both individuals and analyzed them in the context of the V $\beta$ specificity of the M1T1 SAgs. The globally disseminated M1T1 strain harbors the SpeA2, speF, speG, speJ, and SmeZ1 genes. Although these SAgs overlap in their specificity to certain $\mathrm{V} \beta$ elements, they also have unique signature TCR V $\beta$ specificities $(103,114)$. It was therefore of interest to determine if the heightened response of individual "B" to the M1T1 SAgs is related to over-expression of M1T1 SAgs-reacting TCR V $\beta$ elements in this individual repertoire.

The TCR V $\beta$ repertoires of individual " $A$ " and "B" were comparable, with several exceptions (Fig. 3.3). The relative expression of TCR V $\beta$ 1, 3, 5.1, 8 and 11 was higher in the repertoire of the low responder individual " $A$ " than in individual "B", however none of these V $\beta$ elements are specific to the M1T1 SAgs listed above (100, 106). By contrast, expression of TCR V $\beta 8$, which is specific for SmeZ1, was higher in individual A, yet this individual is the low responder. These data are consistent with the conclusion that individual variations in the TCR V $\beta$ repertoire have little or no effect on the magnitude of SAgs responses. Although certain TCR V $\beta$ elements (e.g. V $\beta 6.9,7.3$, $10,15,19,24$ and 25) are not included in our analysis, some of these (V $\beta 10,24$ and 25) are not specific to any known Strep SAgs (Table 1.3). Also, V $\beta 7.3$ is reactive to SpeH, however, this SAg is not produced by the clonal M1T1 strain. Furthermore, V $\beta 6.9$ is specific to SpeG, but it is not a major taraget for this SAg (106). Similarly, V $\beta 15$ is not a major target for SpeA, and V $\beta 15$ and 19 are reactive to SpeF that is disputed as a SAg. 


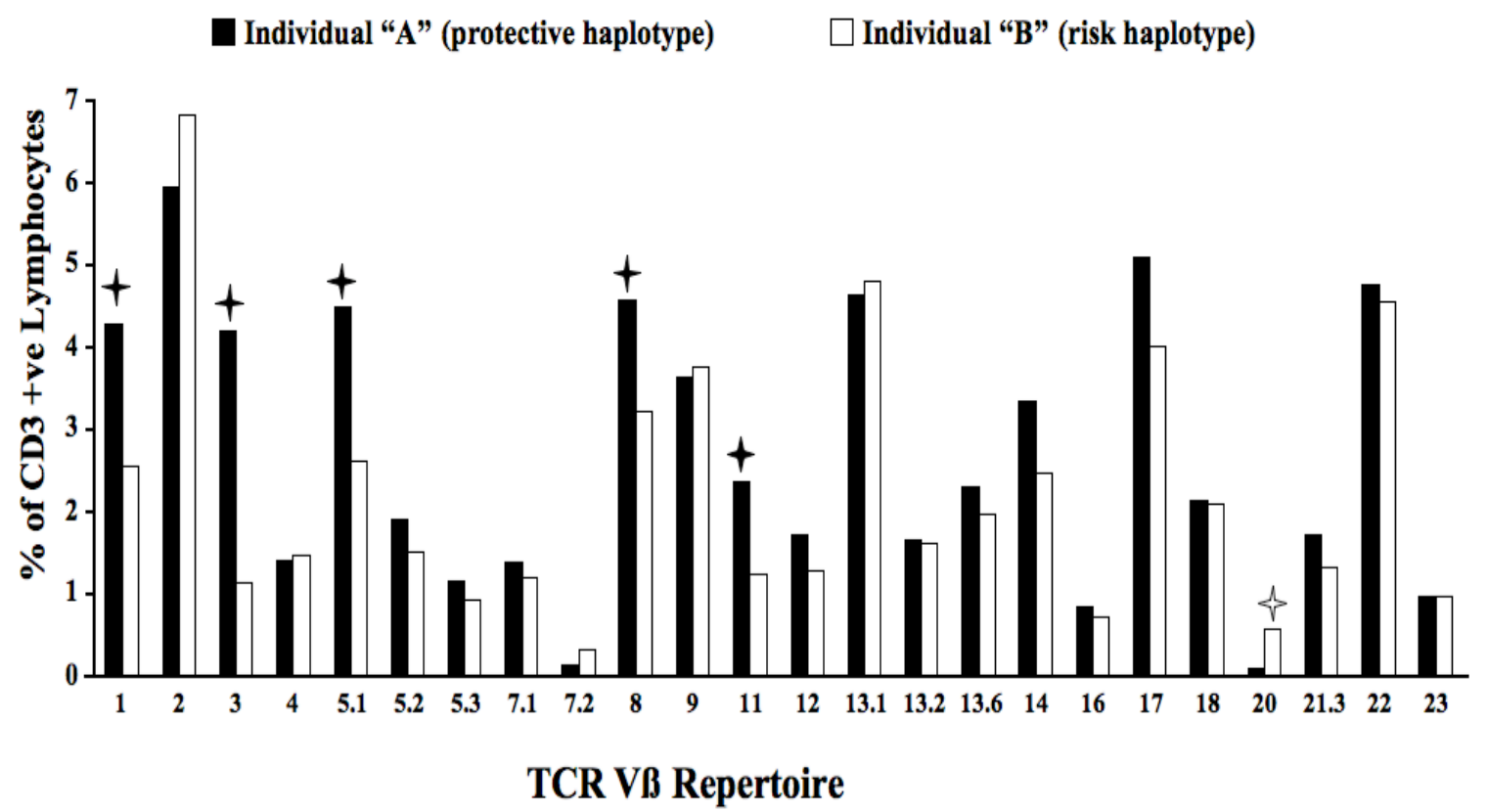

Figure 3.3 Comparison of the TCR V $\beta$ repertoire of individual "A" (protective haplotype) and "B" (risk haplotype).

PBMC from both individuals were stained with fluorescently labeled antibodies against $\mathrm{CD}^{+}$and a panel of antibodies directed to the 24 indicated $\mathrm{V} \beta$ subfamilies, as described in Methods, then analyzed by flow cytometry. Each bar represents the mean of the percentages of cells expressing a specific TCR V $\beta$ subfamily within the $\mathrm{CD} 3^{+}$population. 
In addition, the cross presentation results (mentioned above) exclude variation in the unstudied TCR V $\beta$ elements as a factor contributing to disparate SAg responses.

\section{DR15/DQ6 alleles diminish SAg responses by inducing high levels of IL-10}

In agreement with the proliferation results, cytokine responses to the Strep SAgs were also affected by the type of HLA class II alleles expressed by the presenting APC. The levels of IL-2, IL-6, TNF- $\alpha$, and notably IFN- $\gamma$ and TNF- $\beta$, which are typical SAginduced inflammatory cytokines, mounted by individual "A" PBMC, were significantly lower than by individual "B" ( $p<0.001-0.05 ;$ Fig. 3.4A). Interestingly, however, individual "A" (low responder) produced significantly higher levels of the antiinflammatory cytokine IL-10 than individual "B" ( $p<0.01$; Fig. 3.4A). IL-4 responses were undetectable in either individual (data not shown).

IL-2, IL-6, TNF- $\alpha$, TNF- $\beta$ and IFN- $\gamma$ responses were also significantly lower when the M1T1 SAgs were presented by the B cell line expressing the protective haplotype DR15/DQ6 as compared to that expressing the high-risk DR14/DQ5 haplotype $(p<0.001-0.05$; Fig. 3.4B). Importantly, IL-10 levels were significantly higher in the presence of the DR15/DQ6 expressing cells than in the presence of the DR14/DQ5 haplotype at all time points tested ( $p<0.001-0.05$; Fig. 3.4B).

\section{Exogenous IL-10 diminishes SAgs responses induced by high-risk alleles}

The lower proliferative responses coupled with higher level of the anti inflammatory IL-10 in the presence of the protective DR15/DQ6 alleles compared to the high-risk alleles suggested that SAg presentation by the protective alleles may be 
A $\square$ Individual "A" (protective haplotype) $\square$ Individual "B" (risk haplotype)
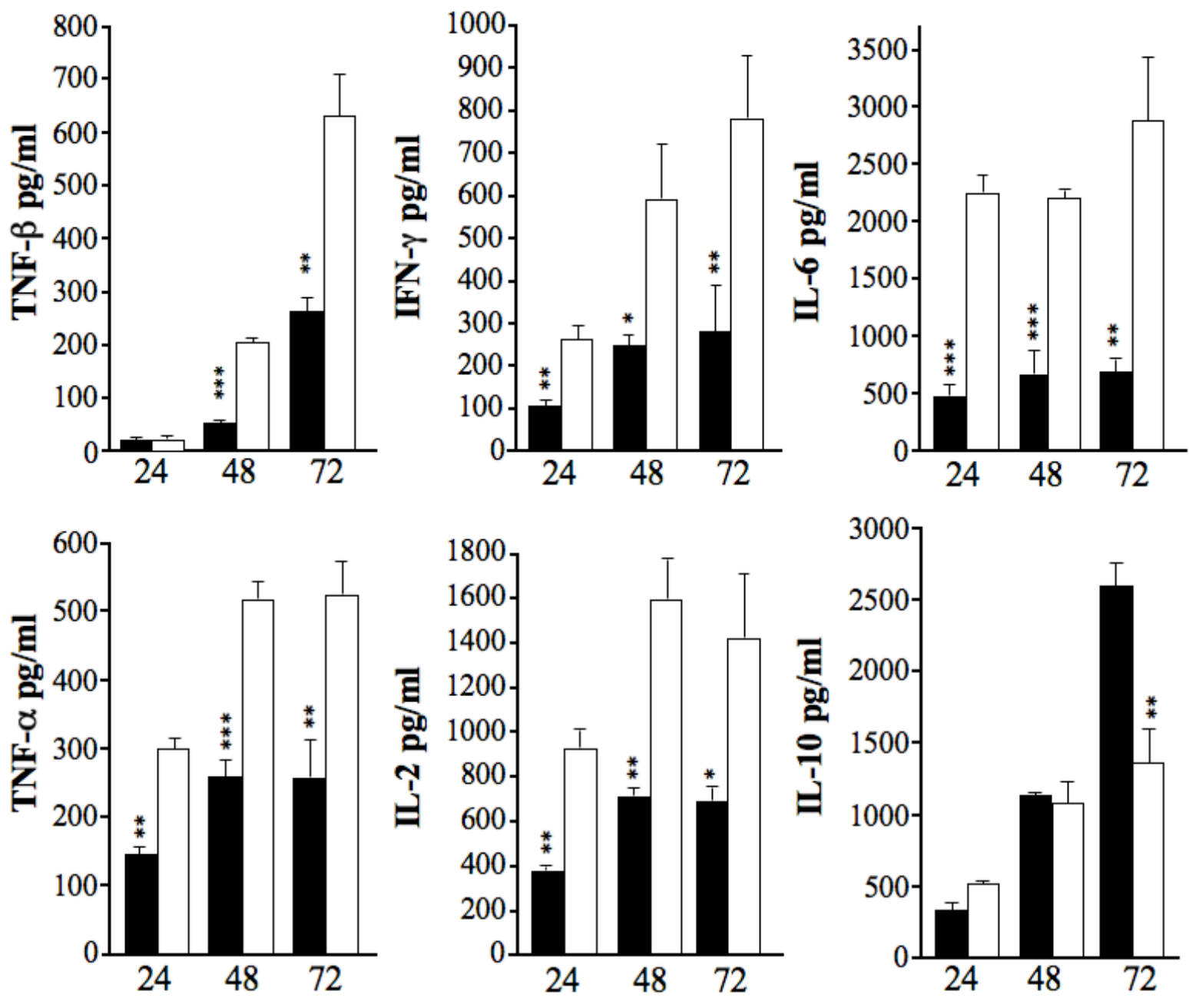

Hours in Culture

Figure 3.4 The HLA type of the APC determines the type and magnitude of cytokine responses to the Strep SAgs.

A. Cytokine responses of PBMC from both individuals to native M1T1 SAgs mixture. Cells $\left(2 X 10^{5} /\right.$ well) were stimulated with the native M1T1 Strep SAg mixture in triplicates, and at the indicated times points, culture supernatants were collected and analyzed for the production of TNF- $\beta$, IFN- $\gamma$, IL- 6 , TNF- $\alpha$, IL-2 and IL-10 $B$. Pure APC-depleted T cells (1X10/well) were stimulated with an optimal dilution of native M1T1 SAgs mixture presented by B cell lines $\left(0.5 \times 10^{5} /\right.$ well $)$ homozygous for either the STSS protective or high risk HLA- Class II haplotypes. Culture supernatants were collected at the indicated times points post stimulation, and analyzed for the production of TNF- $\beta$, IFN- $\gamma$, IL-6, TNF- $\alpha$, IL-2 and IL-10. Each data set represents mean \pm SD.

Significant differences were evaluated by ANOVA $\left(* * * \mathrm{P}<0.001\right.$, $\left.* * \mathrm{P}<0.01,{ }^{*} \mathrm{p}<0.05\right)$. 
B

口DRB1*1501/DQB1*0602 APC (protective) $\square$ DRB1*1401/DQB1*0503 APC (risk)
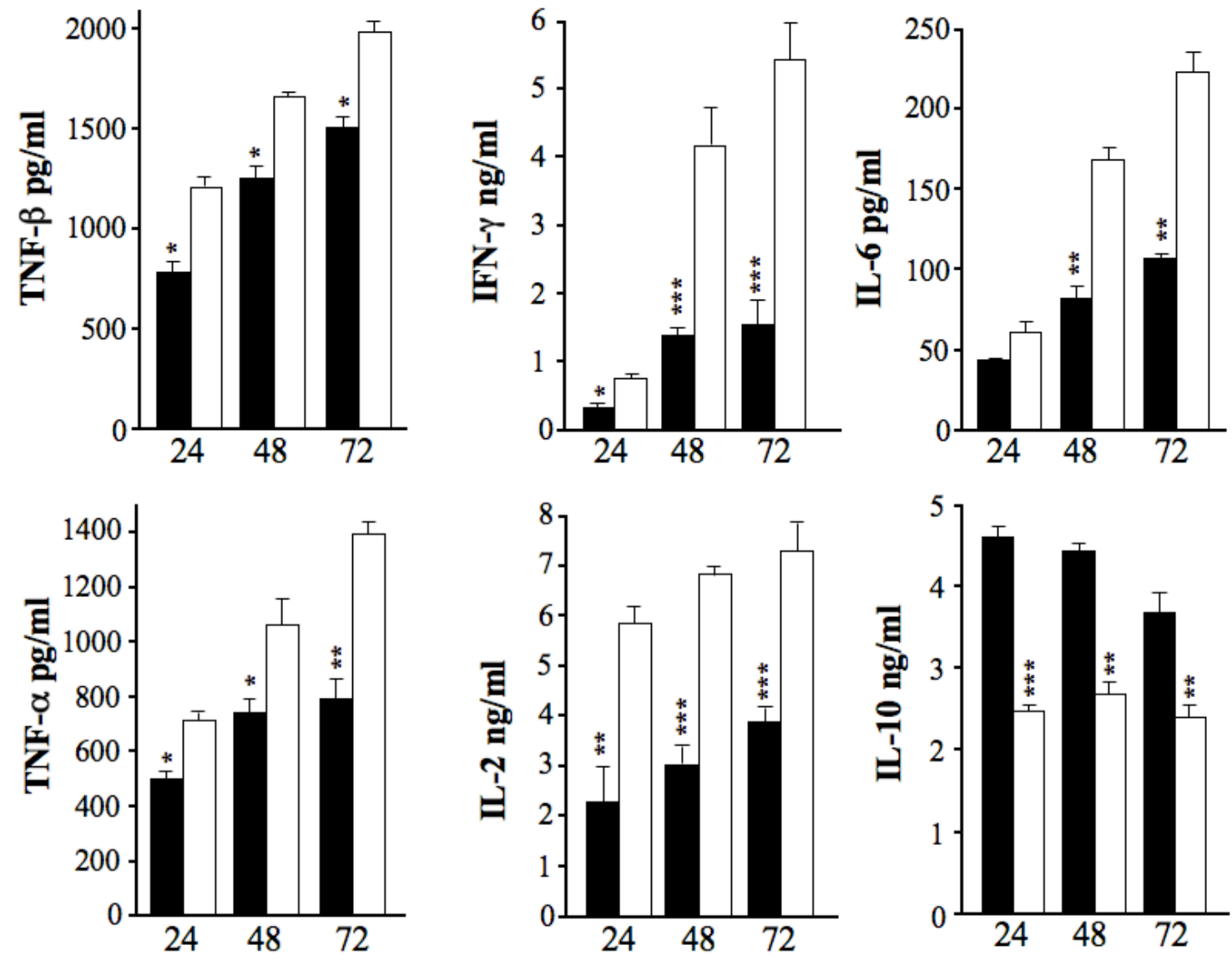

Hours in culture

Figure 3.4 (continued). 
eliciting signals that actively suppress SAgs responses rather than merely eliciting a weak response. We tested this possibility by comparing the proliferative response of individual "B" in the presence or absence of added human rIL-10 (3ng/ml) to that of individual "A" PBMC stimulated with the same Strep SAgs mixture (Fig. 3.5). The chosen concentration of IL1-10 was based on the amount produced by the low responder, individual "A".

As shown in Fig. 3.5, the response of PBMC from the high responder (individual "B") to the Strep SAgs was reduced down to the same level of the low responder (individual "A") when IL-10 was added. Similarly, when pure T cells from either individual were stimulated with the same SAgs in the context of the B cell line homozygous for the DR14/DQ5, the response was comparably elevated in the absence of added IL-10 and similarly reduced in the presence of this regulatory cytokine (Fig. 3.5). Thus, in all combinations and regardless of the T cell donor, higher proliferative responses seen in the presence of the high-risk alleles were significantly diminished by the addition of IL-10 (Fig. 3.5).

\section{Discussion}

The role of HLA allelic variation in modulating host responses to Strep SAgs and determining the outcomes of GAS sepsis has been first identified through epidemiological genetic association studies (275) and later validated biologically, in vitro and in vivo, using HLA transgenic mouse models (319). In this study we showed that HLA class II allelic polymorphism is the main determinant of the type and magnitude of the SAg response and, unlike common belief, we found little to no effect of individual 


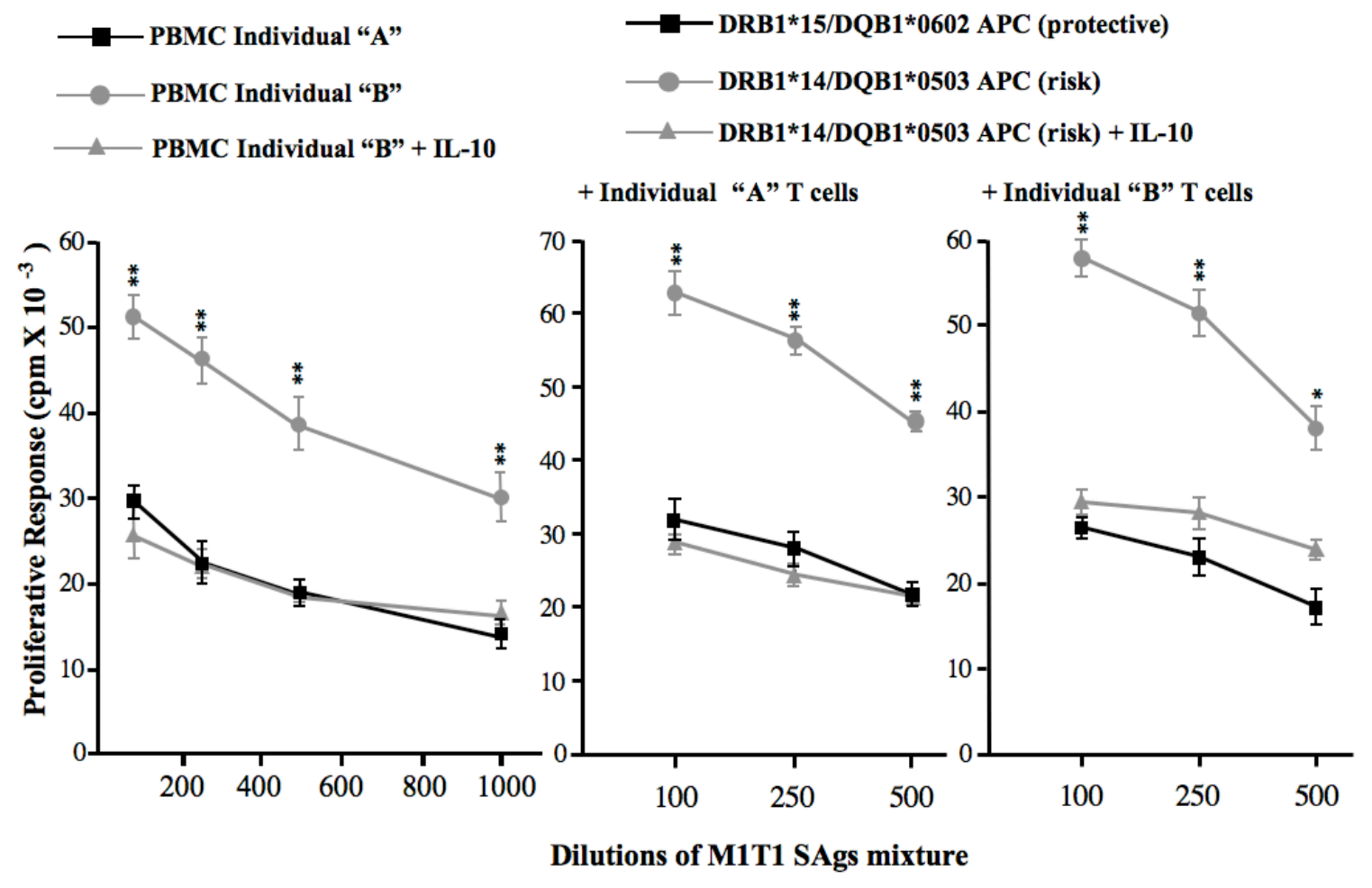

Figure 3.5 Active suppression of Strep SAgs-driven proliferative responses by IL-10.

PBMC from individual "B" (high responder) $\left(2 \mathrm{X} 10^{5} /\right.$ well) were stimulated as described in Figure 3.1 legend in the presence or absence of rIL-10 (3ng/ml). Pure T cells $\left(1 \mathrm{X} 10^{5} /\right.$ well) from individual "A" or "B" were stimulated as described in Figure 3.2 legend with or without rIL-10 (3ng/ml) added to cultures containing B cell lines $\left(0.5 \times 10^{5} /\right.$ well) homozygous for either the STSS protective or high risk HLA Class II haplotypes. PBMC from individual "A" or pure T cells co-cultures with B Cell line expressing the protective haplotype were used as a reference for low response. The data represent the mean and SD of three experiments. ( $* * \mathrm{P}<0.01, * \mathrm{P}<0.05)$. 
variations in TCR V $\beta$ repertoire. This finding is particularly interesting in the context of our earlier studies where we showed that although certain SAgs can interact directly with T cells in the absence of HLA class II presentation, CD28 crosslinking was needed to induce T cells to proliferate $(179,180,209,210)$. These observations had lead us to believe that the requirement for HLA class II molecules in SAg responses is to bring $\mathrm{T}$ cells and APC in close proximity to allow productive interactions between CD28 and B7 molecules as well as other receptors/ligands on both types of cells $(179,180,209,210)$. Several studies have shown that allelic variation in HLA class II molecules can affect the type of signaling transduced through the MHC and TCR receptors and affect the magnitude of SAg response $(172,185,284-288,324,325)$. The data presented here show an even more important role for HLA class II molecules in SAg presentation - different allelic variants not only control the magnitude of SAg responses, but clearly they also affect the overall type of cytokines produced by the SAg stimulated T cells.

In our previous studies, the presentation of SAgs produced by the prevalent M1T1 clonal strain of GAS by the STSS-protective HLA DR15/DQ6 haplotype or by individual alleles on this haplotype elicited significantly lower proliferative and inflammatory cytokine responses than the presentation by haplotypes/alleles associated with high-risk for STSS (DR14/DQ5 halptoype) or haplotypes that are neither associated with-risk nor protection from STSS (neutral haplotypes) $(275,319)$. This was thought to be due to weaker presentation of the SAgs by the protective haplotype. However, the present study shows that presentation by the protective haplotype elicits high levels of IL-10, which possibly contributed to suppression of the inflammatory responses and reduction of $\mathrm{T}$ cell proliferation. Thus, the protective effect of the DR15/DQ6 haplotype may not be an 
entirely passive action as we previously thought. Rather, an active IL-10-mediated suppression of the Strep SAgs-triggered inflammatory responses may play a part in this process. We will further address this hypothesis by using anti-IL-10 Ab in vitro and in vivo in HLA-tg mice infected with prevalent M1T1 GAS strain.

Inflammatory cytokines play a central role in shaping the systemic manifestation of sepsis $(326,327)$. Our previous studies have demonstrated that patients with severe systemic manifestation of GAS sepsis have a propensity to produce high levels of inflammatory cytokine responses in either acute or convalescent phases of infection (275, 276). In agreement with these reports, we observed that PBMC from individual "A" (expressing protective alleles) produced significantly lower pro-inflammatory cytokines than those from individual "B" (expressing high-risk alleles), when both were stimulated with the same SAgs. In the cross-APC presentation studies, we found that the T cells from either individual can mount the same level of response if the SAgs were presented by the same APC from either individual or by B cell lines homozygous for the protective or high risk haplotype. This provides further support for the pivotal role of HLA class II allelic variation in governing SAgs responses and determining the severity of GAS sepsis.

It is well established that SAgs signal through MHC class II and TCR receptors and that these signals elicit a cascade of molecular events that result in the production of high levels of inflammatory cytokines including IL-2, TNF- $\alpha$, TNF- $\beta$, IL-1, IL-6 and IFN- $\gamma$ etc $(276,324,328-331)$. Although several studies have investigated the nature of biochemical signals that ensue SAg interactions with MHC and TCR receptors, few have compared the effect of variability in either receptor $(286,332,333)$. In this study we 
found insignificant contribution of TCR V $\beta$ repertoire variability, but clearly HLA class II allelic variability has a dramatic influence on the magnitude and profile of responses to the same SAgs. We are unaware of any previous studies demonstrating that SAgs elicit differential higher levels of IL-10 than inflammatory cytokine (e.g. IFN- $\gamma$ and TNF- $\beta$ ). From the data presented here, it appears that the ability of SAgs to elicit high levels of IL10 production is only seen when presentation is by the DR15/DQ6 haplotype. At present it is not clear how the interactions of the same SAgs with different alleles can result in such significant difference in the overall response, but we are actively investigating molecular and biochemical events responsible for these stark differences in SAg responses in the presence of distinct HLA alleles.

IL-10 is produced by monocytes and lymphocytes and has multiple effects in immunoregulation and inflammation $(334,335)$. It downregulates the expression of several inflammatory cytokines as well as inducible or constitutive expression of HLA class II and costimulatory molecules $(335,336)$. The role of IL-10 in gram-negative and gram-positive sepsis has been thoroughly examined and it appears that this regulatory cytokine plays a more important role in the latter type of sepsis (337). Administration of IL-10 in several experimental and clinical studies showed benefit $(338,339)$, but the effect can vary considerably depending on the type of infection, the timing of IL-10 administration and the relative levels of other inflammatory and anti-inflammatory mediators. Whereas the initial production of inflammatory in infection is needed to help initiate protective immune responses, uncontrolled overproduction of cytokines like IFN$\gamma$ and TNF- $\alpha$ can be detrimental, causing host-mediated pathogenesis vascular collapse and organ damage (340). On the other hand, overexpression of regulatory cytokines like 
IL-10 may induce overt immunosuppression and thus increase mortality in bacterial sepsis $(341,342)$. Therefore, any imbalance in the production of pro- or antiinflammatory cytokines can lead to pathological consequences $(343,344)$. It would be of interest to measure IL-10 production in STSS patients carrying the STSS protective haplotype (DR15/DQ6) and compare it to those carrying the high-risk (DR14/DQ5 and DR7) alleles. More importantly it will be important to determine the overall balance between the inflammatory and anti-inflammatory cytokines in these patients. Although the vast majority of GAS sepsis patients with the DR15/DQ6 haplotype were protected against the severe form of the disease, few developed STSS (275). It will be of interest, therefore to determine if additional polymorphisms affecting IL-10 production in GAS sepsis can either confer increased protection or increase the risk for STSS. IL-10 expression is regulated by other cytokines and can be affected by polymorphisms within its own gene (345). For example, allelic differences in position -1082 of the IL-10 promoter have been shown to affect its production $(346,347)$.

Although our data support a direct and dominant effect of HLA molecules on SAg-induced responses, we should take into account effects of other polymorphic genes that may also be involved in regulating immune responses to the SAgs as well as to other bacterial components besides the SAgs that are likely to contribute to the pathogenesis of GAS sepsis as well. To this end we have recently adopted a forward genetics approach to investigate the role of multiple interactive genes and pathways in modulating host susceptibility and severity of infection with GAS as well as other pathogens (348) (Abdeltawab, N.F. et al. in press). Eventually, with these combined approaches we aim to translate our findings to the bedside, where it will become possible to predict the 
outcomes and progression of specific infections based on combinations of genetic and soluble biomarkers to allow judicial intervention and customized patient management. 


\section{Chapter 4. Molecular Basis for HLA Class II Differential Presentation of Streptococcal Superantigens Involved in GAS Sepsis}

\section{Introduction}

The frequency of Group A streptococcal severe invasive manifestations, such as streptococcal toxic shock syndrome (STSS) and necrotizing fasciitis (NF), have increased remarkably during the last two decades $(221,292)$. These manifestations are rapidly progressive infections with high mortality rates despite prompt antimicrobial therapy. Group A streptococci (GAS) cause more than 19.7 million cases of severe invasive infections each year worldwide, resulting in over 0.5 million yearly deaths (16).

Despite the presence of over 100 GAS serotypes, the resurgence of such severe systemic diseases (SSD) was associated with emergence of a globally disseminated subclone of M1T1 serotype. This clonal M1T1 strain is characterized by the acquisition prophages encoding distinctive virulence determinants such as the powerful DNase streptodornase 1 (Sda1) and the pyrogenic exotoxin A2 (SpeA2) (6). Moreover, recent studies revealed that M1 protein has superantigenic activities that may explain the overrepresentation of clonal M1T1 among isolates from SSD cases (33).

Although GAS possess a vast array of virulence factors that promote adhesion, invasion, survival and immune evasion, the systemic effects seen in patients with SSD are primarily triggered by inflammatory mediators induced in response to streptococcal pyrogenic exotoxins (Spes) $(9,318)$. Clonal M1T1 GAS express numerous Spes, including SpeA2, SpeF, SpeG, SpeJ, and streptococcal mitogenic exotoxin Z1 (SmeZ1) $(100,124,143,166)$. Spes are superantigens (SAgs), which are by far the most potent 
mediators of pro-inflammatory responses, and have therefore been implicated as pivotal players in the pathogenesis of STSS $(214,349)$. SAgs bind simultaneously to HLA class II molecules on antigen presenting cells (APC) and to distinct variable elements of TCR $\mathrm{V} \beta$ chain on T cells (98), bridging these critical immune receptors and triggering excessive activation of both cell types resulting in massive release of inflammatory cytokines. Class II allelic variation has been shown to affect SAg responses $(275,287-$ 289, 319). Evidence for SAgs association with SSD came from investigations demonstrating TCR V $\beta$ repertoire alterations in patients during acute infection (102), detection of SAgs in patients sera and tissue biopsy $(101,225)$ and animal models where SAgs triggered systemic manifestations similar to those observed in human infections $(226,227,297,319)$.

A direct correlation between pro-inflammatory cytokines level and invasive GAS infection severity was observed $(101,276)$. Patients' propensity to produce higher or lower cytokine levels correlated with the development of severe or non-severe invasive infection, suggesting involvement of certain host immunogenetic factors in regulating SAgs responses (276). This hypothesis was also supported by our epidemiological study demonstrating that HLA class II polymorphism influenced the outcome of invasive GAS infections (275). HLA class II haplotypes associated with protection from SSD presented GAS SAgs in a way that resulted in significantly lower cytokines and proliferative responses. This was partly explained by our recent finding, which demonstrated that the protective haplotype preferentially induced higher levels of the anti-inflammatory cytokine IL-10 (chapter 3). However, SAgs interaction with the immune system is multifaceted. We undertook this study to get a better understanding of how HLA class II 
polymorphism affects SAgs responses and modulates GAS infection outcome. We explored the molecular basis of the differential interaction between HLA class II and Strep SAgs.

In this report, we generated a battery of bare lymphocyte syndrome (BLS) cell lines expressing single HLA class II alleles, and used it to delineate the contribution of individual class II alleles to Strep SAgs responses. Because of the quantitative nature of our studies, it is critical to ensure coordinated and balanced expression of both class II $\alpha$ and $\beta$ subunits on BLS cells. Thus, we used a picornavirus-like 'self-cleaving' $2 \mathrm{~A}$ peptide sequences to link class II $\alpha$ - and $\beta$-chains. The extremely rare 2 A peptide conserved motif (2A: Asp-Val/Ile-Glu-X-Asn-Pro-Gly-[//]-2B: Pro, Fig. 1) impairs normal peptide bond formation between the Gly and Pro without affecting the translation of $2 \mathrm{~B}$ (the protein on the C-terminal of the $2 \mathrm{~A}$ peptide) (350). The 2A peptide, also known as cis-acting-hydrolase, mediates this cleavage through posttranslational processing that requires no viral or host proteinase (351).

\section{Materials and Methods}

\section{GAS superantigens}

Recombinant SpeA2, SpeG, SpeJ and SmeZ1 were expressed in E. coli and purified as His fusion proteins as described previously (323). The purified rSAgs were treated with polymyxin B agarose (Boehringer Mannhiem, Indianapolis, IN) to remove any contaminating endotoxin. 
Native secreted SAgs mixture was prepared from the supernatant of overnight cultures of GAS clinical isolates by ethanol precipitation followed by extensive dialyses, as detailed elsewhere (319).

\section{Generation of HLA class II multicistronic vectors}

Genes encoding class II $\alpha$ and $\beta$ chains were cloned from B lymphoblastoid cell lines (BLCL) homozygous for the appropriate haplotype. 2A peptide-linked constructs were generated by recombinant PCR as detailed elsewhere (352), using scheme shown in Figure 4.1 and the primers in Table 4.1. The constructs were cloned into the retroviral vector pHSPG. A trinucleotide GSG spacer was included between class II $\beta$ chain and the 2A peptide to maximize cleavage efficiency. Since the HLA DR $\alpha$-chain is nonpolymorphic, the DRB1*1401-P2A-DRA1*0101 construct was generated with Bstz17I recognition sequence inserted between DR $\beta$ chain and the P2A peptide, so that it could be used together with EcoRI to digest DRB1*1401 out of the construct and replace it with $\beta$ chain for the other DR alleles.

\section{Virus preparation, transduction and cell sorting}

The retroviral vector pHSPG has GFP marker gene driven by phosphoglycerate kinase promoter (pGK). To prepare viral particles, pHSPG-class II allele plasmids were co-transfected into 293T cells with gag/pol and VSVg vectors by the calcium phosphate method. Viral supernatants were collected 48 and $72 \mathrm{~h}$ post transfection and $0.48 \mu$ filtered. Transductions by spin inoculation were performed by suspending BLS cells in fresh viral supernatants at $0.5 \times 10^{6} / \mathrm{ml}$ in the presence of $8 \mu \mathrm{g} / \mathrm{ml}$ polybrene 


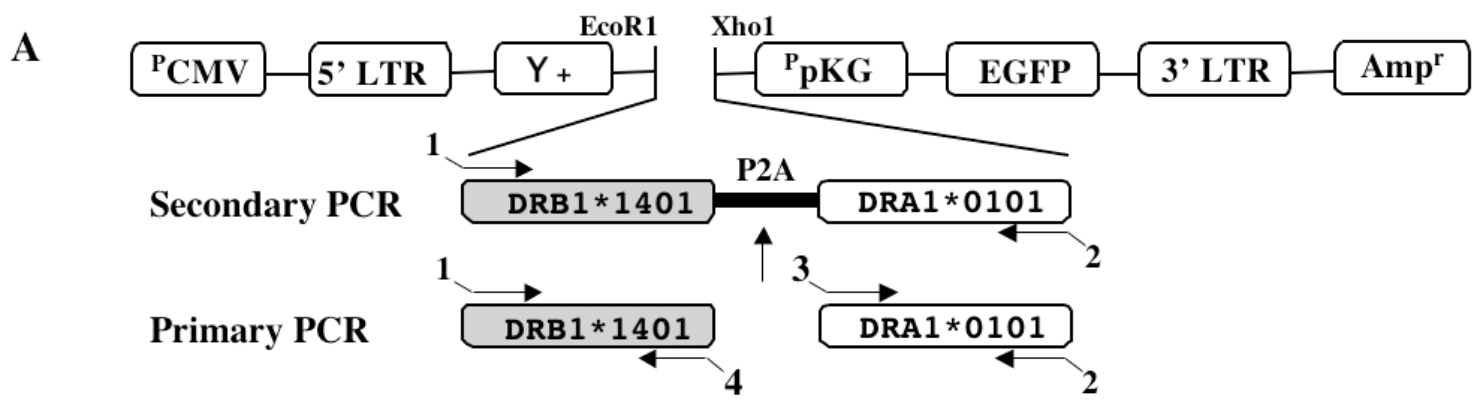

B

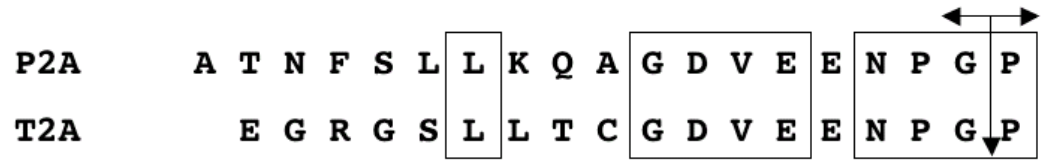

C

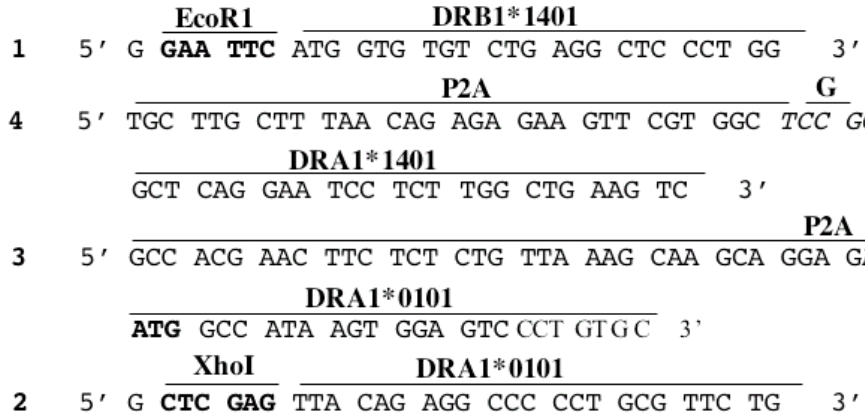

Figure 4.1 Generation of 2A-linked HLA Class II constructs.

A. Schematic of the pHSPG retroviral vector containing the 2A linked Class II constructs, DRB1-P2A-DRA1 and DQB1-T2A-DQA1 produced by recombinant PCR. The constructs were cloned into a CMV-based retroviral vector that contains enhanced GFP driven by phosphoglycerate kinase (PG) promoter. The position and direction of primers used is shown. B. Amino acid sequence of 2A peptide of porcine teschovirus-1 (P2A) and Thosea asigna (T2A). Conserved residues are boxed. The cleavage point between the $2 \mathrm{~A}$ and $2 \mathrm{~B}$ peptides, and thus the $\mathrm{NH}_{2}$ - and $\mathrm{COOH}$-terminal cisterons, is indicated by the arrow. $C$. The oligonucleotide sequences used for generation of DRB1*1401-P2A-DQA1*0101, as an example for primers used for PCR, are shown. 
Table 4.1 Primers used to clone different class II alleles and to link the $\alpha$ - and $\beta$-chain by 2A peptide.

\begin{tabular}{|c|c|c|}
\hline Gene & & Primer sequence \\
\hline \multirow[t]{2}{*}{ DRA $1 * 0101$} & Fwd & $\begin{array}{l}\text { 5'-GCC ACG AAC TTC TCT CTG TTA AAG CAA GCA GGA GAC GTG } \\
\text { GAA GAA AAC CCC GGT CCC ATG GCC ATA AGT GGA GTC CCT } \\
\text { GTG C-3' }\end{array}$ \\
\hline & Rev & 5'-G CTC GAG TTA CAG AGG CCC CCT GCG TTC TG-3' \\
\hline DRB $1 * 1401$ & $\begin{array}{l}\text { Fwd } \\
\text { Rev }\end{array}$ & $\begin{array}{l}\text { 5'-G GAA TTC ATG GTG TGT CTG AGG CTC CCT GG-3' } \\
\text { 5'-TGC TTG CTT TAA CAG AGA GAA GTT CGT GGC TCC GGA ACC } \\
\text { GTA TAC GCT CAG GAA TCC TCT TGG CTG AAG TC-3' }\end{array}$ \\
\hline $\mathrm{DRB} 1 * 1501$ & $\begin{array}{l}\text { Fwd } \\
\text { Rev }\end{array}$ & $\begin{array}{l}\text { 5'-G GAA TTC ATG GTG TGT CTG AAG CTC CCT GG-3' } \\
\text { 5'-G GTA TAC GCT CAG GAA TCC TGT TGG CTG AAG-3' }\end{array}$ \\
\hline DRB $1 * 0402$ & $\begin{array}{l}\text { Fwd } \\
\text { Rev }\end{array}$ & $\begin{array}{l}\text { 5' G GAA TTC ATG GTG TGT CTG AAG TTC CCT GGA G-3' } \\
\text { 5'-G GTA TAC GCT CAG GAA TCC TGT TGG CTG AAG-3' }\end{array}$ \\
\hline DQB1*0602 & $\begin{array}{l}\text { Fwd } \\
\text { Rev }\end{array}$ & $\begin{array}{l}\text { 5'-G GAA TTC ATG TCT TGG AAA AAG GCT TTG CGG-3' } \\
\text { 5'-CC GCA TGT TAG CAG ACT TCC TCT GCC CTC ACC GCT ACC } \\
\text { GTG CAG GAG CCC TTT CTG ACT CCT G-3' }\end{array}$ \\
\hline DQB $1 * 0503$ & $\begin{array}{l}\text { Fwd } \\
\text { Rev }\end{array}$ & $\begin{array}{l}\text { 5'-G GAA TTC ATG TCT TGG AAG AAG TCT TTG CGG ATC C-3' } \\
\text { 5'-CC GCA TGT TAG CAG ACT TCC TCT GCC CTC ACC GCT ACC } \\
\text { GTG CAG AAG CCC TGC TGG TGG-3' }\end{array}$ \\
\hline \multirow[t]{2}{*}{ DQA $1 * 0104$} & Fwd & $\begin{array}{l}\text { 5'-GAG GGC AGA GGA AGT CTG CTA ACA TGC GGT GAC GTC } \\
\text { GAG GAG AAT CCT GGC CCA ATG ATC CTA AAC AAA GCT CTG } \\
\text { CTG CTG G-3' }\end{array}$ \\
\hline & $\begin{array}{l}\text { Rev } \\
\text { Fwd }\end{array}$ & $\begin{array}{l}\text { 5'-G CTC GAG TCA CAA TGG CCC TTG GTG TCT GG-3' } \\
5^{\prime}-\text { G GAA TTC ATG TCT TGG AAA AAG GCT TTG CGG-3' }\end{array}$ \\
\hline DQB $1 * 0202$ & Rev & $\begin{array}{l}\text { 5'-CC GCA TGT TAG CAG ACT TCC TCT GCC CTC ACC GCT ACC } \\
\text { GTG CAG GAG CCC TTT CTG ACT CCTG-3' }\end{array}$ \\
\hline \multirow[t]{2}{*}{ DQA $1 * 0201$} & Fwd & $\begin{array}{l}\text { 5'-GAG GGC AGA GGA AGT CTG CTA ACA TGC GGT GAC GTC GAG } \\
\text { GAG AAT CCT GGC CCA ATG ATC CTA AAC AAA GCT CTG ATG } \\
\text { CTG GG-3 }\end{array}$ \\
\hline & Rev & 5'-G CTC GAG TCA CAA GGG CCC TTG GTG TCT GG-3 \\
\hline
\end{tabular}

(1) Italicized, underlined sequence indicates the GSG spacer.

(2) Bold sequences are the restriction enzymes recognition sequences.

(3) Bold underlined sequence indicates the start of the gene on 2A peptide C-terminus.

(4) The primers for DRB $1 * 0701$ are the same as DRB $1 * 1501$ primers.

(5) The primers for DQA $1 * 0102$ are the same as DQA1*0104 primers.

(6) The primers for DQB1*0202/DQA1*0102 are the same as DQB1*0301/DQA1*0501 primers. 
followed by centrifugation at $2000 \mathrm{Xg}$ for $3 \mathrm{~h}$. Transduction was repeated on two consecutive days to increase infection efficiency. Following one week of culture, stably transduced cells were sorted for GFP expression. FACS analysis showed that GFP and class II expression is $95 \%$ stable for at least two months following sorting (not shown).

\section{Cells}

We used Ficoll-Hypaque density-gradient centrifugation to isolate the peripheral blood mononuclear cells (PBMC) from healthy donors blood. APC-depleted T cells were prepared by subjecting the recovered PBMC to one cycle of erythrocyte rosetting. These enriched T cells were further purified by using the human pan $\mathrm{T}$ cell isolation kit and autoMACS according to the manufacturer's instructions (Miltenyi Biotec, Auburn, CA). To remove any residual APC, we did overnight adherence to polystyrene plate at $37^{\circ} \mathrm{C}$ in RPMI 1640 supplemented with 10\% (v/v) heat-inactivated FBS, 4 mM L-glutamine, 25 $\mathrm{mM}$ Hepes, and $50 \mathrm{U} / \mathrm{ml}$ penicillin/ 50 $\mathrm{g} / \mathrm{ml}$ streptomycin (RPMI complete medium). The purity of T-cells was confirmed phenotypically by flow cytometry and functionally by the lack of response to PHA and/or to rSAgs. Experiments in which purified T cells (cultured alone without any APC) showed a response to PHA or SAgs were discarded.

A panel of EBV transformed B lymphoblastoid cell lines (BLCL) homozygous for HLA class II haplotypes prevalent in areas with high STSS incidence (275) (Table 4.2) were tested together with untransduced BLS cells (HLA class II negative cells) or BLS cells transduced with single HLA DR or DQ alleles (Table 4.3). These cells were used as APC for presenting Strep SAgs. To block cellular division, the cells were treated with mitomycin C (Sigma) $50 \mu \mathrm{g} / 5 \times 10^{7}$ cell $/ \mathrm{ml}$ for $20 \mathrm{~min}$ at $37^{\circ} \mathrm{C}$, 
Table 4.2 Human B lymphoblastoid cell lines expressing prevalent HLA class II haplotypes.

\begin{tabular}{lccccc}
\hline Name & DRB1* & DQB1* & DQA1* & DQ MFI & DR MFI \\
\hline MGAR $^{(b)}$ & 0102 & 0501 & 01012 & $523 \pm 15$ & $2471 \pm 167$ \\
HHKB & 0301 & 0201 & 0501 & $710 \pm 49$ & $2376 \pm 154$ \\
JVM & 0401 & 0301 & 0301 & $560 \pm 49$ & $2243 \pm 147$ \\
VAVY & 0401 & 0302 & 03 & $590 \pm 39$ & $2714 \pm 62$ \\
MADURA & 07 & 03032 & 0201 & $678 \pm 38$ & $2111 \pm 126$ \\
PMG0705 & 0701 & 0202 & 0201 & $754 \pm 50$ & $2221 \pm 149$ \\
WBD001816 & 0801 & 0402 & 0401 & $614 \pm 40$ & $2574 \pm 180$ \\
$1349-8636$ & 1102 & 0301 & 0501 & $650 \pm 34$ & $2407 \pm 126$ \\
BM14 $_{\text {MOU }}^{(\mathrm{c})}$ & 1301 & 0603 & 0103 & $605 \pm 39$ & $2459 \pm 160$ \\
31227 ABO $^{(\mathrm{d})}$ & 1501 & 0602 & 0102 & $583 \pm 21$ & $2308 \pm 83$ \\
\hline
\end{tabular}

a The cells lines were analyzed by flow cytometry for DR and DQ expression level that is assessed by mean fluorescence intensity. Analysis was done with each experiment. The data are presented as mean $\pm \mathrm{SD}$

Cells express alleles associated with

(b) protection from STSS and NF.

(c) predisposition to STSS and NF.

(d) predisposition to STSS. 
Table 4.3 BLS cells transduced with different HLA class II DR or DQ alleles a .

\begin{tabular}{lccc}
\hline Allele designation & $\begin{array}{c}\text { Serological } \\
\text { designation }\end{array}$ & DQ MFI & DR MFI \\
\hline DRB $1 * 0401 / \mathrm{DRA} 1 * 0101$ & DR4 & - & $1655 \pm 142$ \\
$\mathrm{DRB} 1 * 0701 / \mathrm{DRA} 1 * 0101$ & DR7 & - & $1357 \pm 98$ \\
$\mathrm{DRB} 1 * 1401 / \mathrm{DRA} 1 * 0101$ & DR14 & - & $1534 \pm 112$ \\
$\mathrm{DRB} 1 * 1501 / \mathrm{DRA} 1 * 0101$ & DR15 & - & $1852 \pm 168$ \\
$\mathrm{DQB} 1 * 0202 / \mathrm{DQA} 1 * 0201$ & $\mathrm{DQ} 2$ & $765 \pm 57$ & - \\
$\mathrm{DQB} 1 * 0301 / \mathrm{DQA} 1 * 0501$ & DQ7 & $628 \pm 68$ & - \\
$\mathrm{DQB} 1 * 0503 / \mathrm{DQA} 1 * 0104$ & DQ5 & $702 \pm 57$ & - \\
$\mathrm{DQB} 1 * 0602 / \mathrm{DQA} 1 * 0102$ & DQ6 & $844 \pm 77$ & - \\
\hline
\end{tabular}

${ }^{a}$ BLS cell lines were analyzed for the HLA DR or DQ expression level as determined by mean fluorescence intensity (MFI). Analysis was done with each experiment and the data are presented as mean $\pm \mathrm{SD}$. 
extensively washed with HBSS then suspended in RPMI complete medium at a final concentration of $1 \times 10^{6} / \mathrm{ml}$. The mitomycin $\mathrm{C}$ treatment efficacy was confirmed by minimal $\left[{ }^{3} \mathrm{H}\right]$ thymidine uptake compared to untreated cells.

\section{Assessment of HLA DQ and DR expression level}

Using flow cytometry, we analyzed the expression level of HLA DR and DQ molecules on the eleven BLCL homozygous for prevalent Class II haplotypes as well as on the generated cell lines expressing single HLA DR or DQ alleles. This is essential to normalize the data for any difference in class II expression level. When variable expression level was observed, the data were mulitplied by a factor $=$ highest MFI/MFI for each cell line. We used monoclonal antibody (mAb) labeled with phycoerythrin (PE) (Catalog. No. 347367, BD) specific to HLA-DR and primary unlabeled mAb specific to HLA-DQ (MCA379G, serotec) followed by PE-labeled secondary Ab (STAR12A, serotec). Analysis was conducted on a FACSCalibur (Becton Dickinson).

\section{T cell proliferation assays}

Pure T cells ( $1 \mathrm{X} 10^{5} /$ well; 96-well plate), from different healthy donors, were cocultured with mitomycin C-treated B cell lines $\left(0.5 \mathrm{X} 10^{5} /\right.$ well) expressing the prevalent HLA class II haplotypes (Table 2) or single class II alleles (Table 3) in RPMI complete medium. Cells were stimulated with an optimal dilution of partially purified native secreted M1T1 SAgs mixture from clonal M1T1 clinical isolate, individual rSAgs (1 $\mathrm{ng} / \mathrm{ml}$ or $10 \mathrm{ng} / \mathrm{ml})$ or PHA $(1 \mu \mathrm{g} / \mathrm{ml})$. After $72 \mathrm{~h}$ incubation, the cultures were pulsed with $1 \mu \mathrm{Ci} /$ well $\left[{ }^{3} \mathrm{H}\right]$ thymidine, harvested and counted as previously described $(319,323)$. All 
samples measurements were performed in triplicates or quadruplicates, and the data were presented as mean cpm $\left[{ }^{3} \mathrm{H}\right]$ thymidine uptake $\pm \mathrm{SD}$.

\section{Cytokine assays}

Lymphocytes were cultured as described above. Culture media were collected from the individual wells after 24,48 , and $72 \mathrm{~h}$ of stimulation, cleared of cells by centrifugation, and stored at $-80{ }^{\circ} \mathrm{C}$ for subsequent analysis. The cytokines IL-2, IL-4, IL-6, IL-10, IFN- $\gamma$, TNF- $\alpha$ were simultaneously quantified by using the human cytokine LINCOplex kit (Linco Research Inc., St. Charles, MO). TNF- $\beta$ was analyzed by ELISA kit from R\&D Systems (Minneapolis, MN). The assays were performed according to the manufacturer's instructions, and the concentrations were calculated from standard curves run in parallel with the test samples. Data are presented as mean concentration \pm SD.

\section{SAgs labeling and binding assay}

rSpeG, rSpeJ and rSmeZ1 were labeled with Alexa Fluor 647 according to manufacturer's instructions (Molecular Probes, Eugene, OR). Binding assays were performed by incubating $10^{6}$ cells/tube with serial two-fold dilutions (1nM-1 $\left.1 \mu \mathrm{M}\right)$ of labeled rSAg for $4 \mathrm{~h}$ at $4{ }^{\circ} \mathrm{C}$ or $37^{\circ} \mathrm{C}$. After incubation, cells were washed and examined for bound SAg by flow cytometry using FACSCalibur. A total of 30000 cells within the healthy lymphocyte gate were analyzed. SAg binding was assessed as mean fluorescence intensity (MFI) of cells. 


\section{Calcium mobilization studies}

Changes in $\mathrm{T}$ cells intracellular calcium $\left[\mathrm{Ca}^{2+}\right]_{\mathrm{i}}$ were monitored by flow cytometry using cells loaded with the $\mathrm{Ca}^{2+}$-sensitive fluorescent dye, indo-1. Indo-1 loading was done by incubating Jurkat or purified T cells $\left(3 \mathrm{X} 10^{6}\right.$ cells $\left./ \mathrm{ml}\right)$ with $3 \mu \mathrm{M}$ indo- $1 / \mathrm{AM}$ (Molecular Probes) in RPMI complete medium with $5 \% \mathrm{FBS}$ at $37^{\circ} \mathrm{C}$ for $45 \mathrm{~min}$. The indo-1 loaded cells were washed with HBSS, resuspended at $10^{6}$ cells $/ \mathrm{ml}$ in serum freeRPMI complete medium and warmed to $37^{\circ} \mathrm{C}$ prior to stimulation. BLS cells expressing different DR or DQ alleles (1X10\%/ml RPMI complete medium) were incubated overnight with $0.5 \mu \mathrm{g} / \mathrm{ml} \mathrm{rSmeZ1}$ at $37^{\circ} \mathrm{C}$. Baseline fluorescence ratios were collected for $45 \mathrm{sec}$, followed by stimulation with SmeZ1-loaded APC. Samples were analyzed using LSR II flow cytometer (BD) with excitation at $350 \mathrm{~nm}$ and relative ratios of fluorescence emitted at 450 and $530 \mathrm{~nm}$ were monitored over time. The acquired data were analyzed with FlowJo kinetics platform (Tree Star, Inc. Ashland, OR).

\section{Statistical analysis}

Analysis of variance (ANOVA) or a two-tailed Student's $t$ test was used to determine the significance of the data. A $p$ value of $<0.05$ was considered significant.

\section{Results}

\section{Relative effects of prevalent HLA class II haplotypes on M1T1 SAgs responses}

To determine how allelic differences between HLA class II molecules affect the immune response to M1T1 GAS SAgs, we analyzed the presentation of native SAgs 
mixtures by a panel of eleven BLCL homozygous for HLA class II haplotypes listed in Table 2 that are prevalent in Ontario Canada where increasing incidence of Strep SSD cases has been reported $(20,353)$. The SAg mixture was presented by this panel to pure APC-depleted T cells prepared from healthy donors. The highest response was in the presence of cells expressing the two haplotypes associated with high-risk for STSS HLA DRB1*1401/DQB1*0503 and HLA DRB1*0701/DQB1*0201 while the lowest response was in the presence of cells expressing the protective HLA DRB1*1501/DQB1*0602 haplotype (Fig 4.2A). Significantly higher proliferative responses were detected in the presence of cells expressing not only high-risk but also neutral haplotypes HLA DRB1*0401/DQB1*0301 and HLA DRB1*0401/DQB1*0302 (neither associated with high-risk nor protection from STSS) as compared to the cell expressing the protective haplotype ( $\mathrm{p}<0.001-0.05$, Fig 4.2A). The observed variations in SAg stimulation could not be attributed to the level of surface expression of class II molecules on the cells, since flow cytometric analysis of these APC showed equivalent levels of HLA DR or DQ alleles (Table 4.2). Interestingly, the presentation of the M1T1 SAgs by the NF protective haplotype HLA DRB1*0301/DQB1*0201 was higher than by the NF risk haplotype HLA DRB1*1102/DQB1*0301, supporting previous reports that these two severe manifestations of invasive GAS infections differ in their pathogenic mechanisms. The observed different responses were SAg-specific since all cell lines promoted comparable PHA responses (Fig. 4.2B).

In the course of in vivo infection, one or more of M1T1 SAgs may be produced. Therefore, we investigated the presentation pattern of individual M1T1 rSAgs by the same panel of BLCL homozygous for prevalent class II haplotypes (Table 2), 

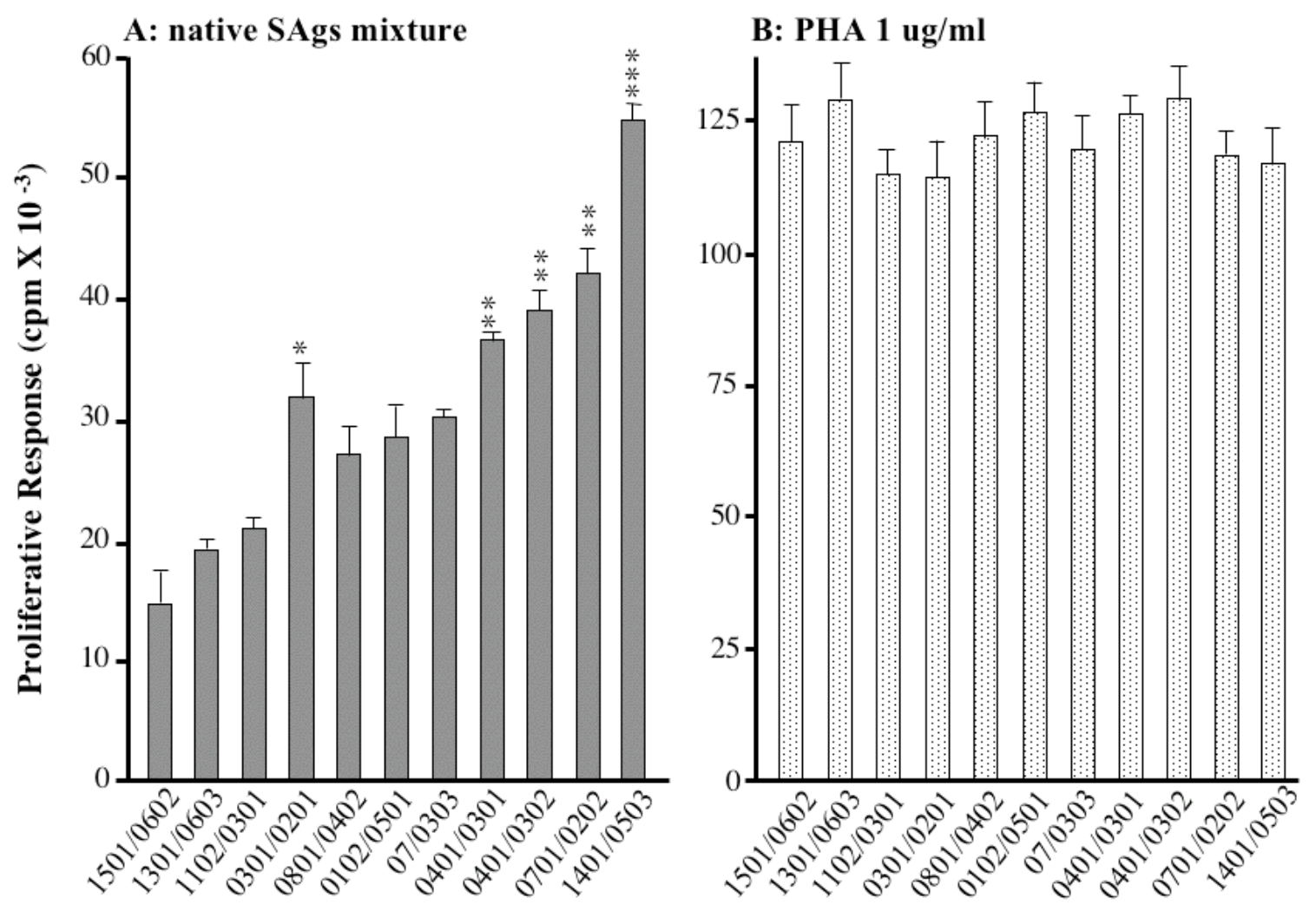

Figure 4.2 Differential presentation of clonal M1T1 SAgs by prevalent HLA class II haplotypes.

Pure APC-depleted T cells (1X10\% $/$ well) from healthy donors were co-cultured with mitomycin C-treated BLCL $\left(0.5 \times 10^{5} /\right.$ well) homozygous for the indicated class II DRB $1 * / D Q B 1 *$ haplotypes. The cells were stimulated with: A. An optimal dilution of native secreted SAgs mixture (1:500), B. PHA (1ug/ml), C. rSpeA2, D. rSpeG, $E$. rSpeJ or $F$. rSmeZ1 $(10 \mathrm{ng} / \mathrm{ml}$, for all tested $\mathrm{rSAg})$. Proliferative responses were determined by $\left[{ }^{3} \mathrm{H}\right]$ thymidine uptake. The data represent mean $\mathrm{cpm} \pm \mathrm{SE}$ of three independent experiments. Significant differences were tested by ANOVA $(* * * \mathrm{P}<0.001$, $* * \mathrm{P}<0.01, * \mathrm{P}<0.05)$. 

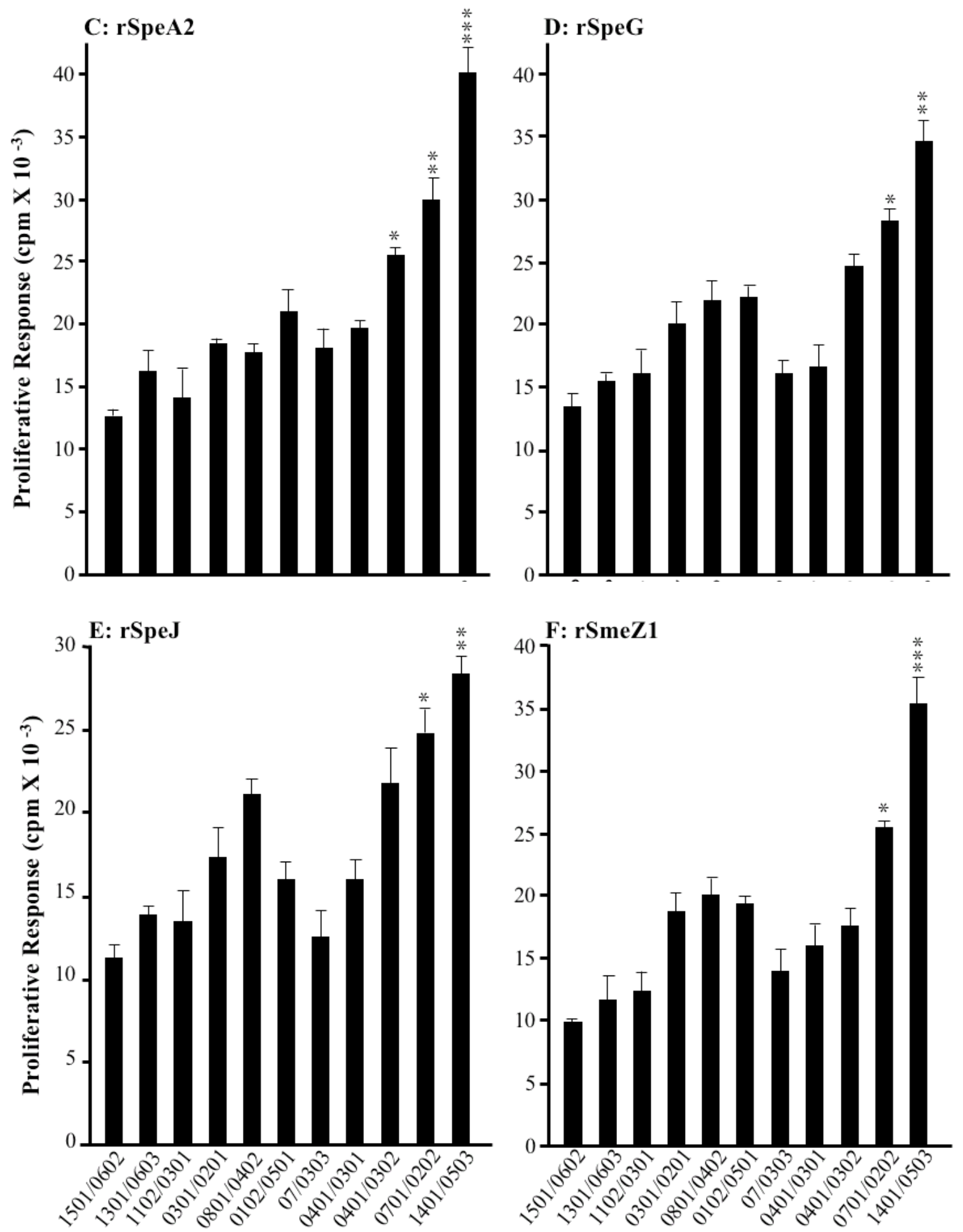

Figure 4.2 (continued). 
using pure $\mathrm{T}$ cells from several healthy volunteers. In agreement with the results for native SAgs mixture, proliferative responses to all tested individually rSAgs were significantly higher when presented by APC homozygous for the STSS high-risk haplotypes DR14/DQ5 or DR7/DQ2 than by the APC homozygous for the STSSprotective haplotype DR15/DQ6 (p<0.001-0.05; Fig .4.2C-F). Thus, supporting our findings with the mixture of native purified SAgs and confirming that the observed differential SAg response was not due to any other component that might have contaminated the native partially purified SAg mixture. Reproducibly, presentation of all M1T1 rSAgs by DR3/DQ2 NF-protective haplotype resulted in higher proliferative responses than by the NF high-risk haplotype DR11/DQ8 confirming that NF, unlike STSS, is not SAg-mediated disease.

\section{Preferential presentation of individual M1T1 rSAgs by HLA DR or DQ alleles}

HLA class II molecules are inherited together and expressed as either homozygous or heterozygous sets of two DP, DQ and DR molecules. Therefore, it is impossible to test the effect of single HLA class II alleles polymorphisms on the inflammatory response to a particular SAg. To achieve this goal we analyzed the presentation of rSpeA2, rSpeG, rSpeJ and rSmeZ1 by BLS cells transduced with distinct HLA DR or DQ alleles (Table 3), using APC-depleted T cells from healthy individuals. For the HLA class II alleles and rSAgs studied, the HLA DR14 or DQ5 consistently promoted significantly higher SAg-induced proliferative responses than did the HLA DR15 or DQ6, respectively ( $\mathrm{p}<0.01-0.05$, Fig. 4.3). Additionally, for the DR alleles, HLA DR14 was always associated with the highest responses, followed by HLA DR7; 

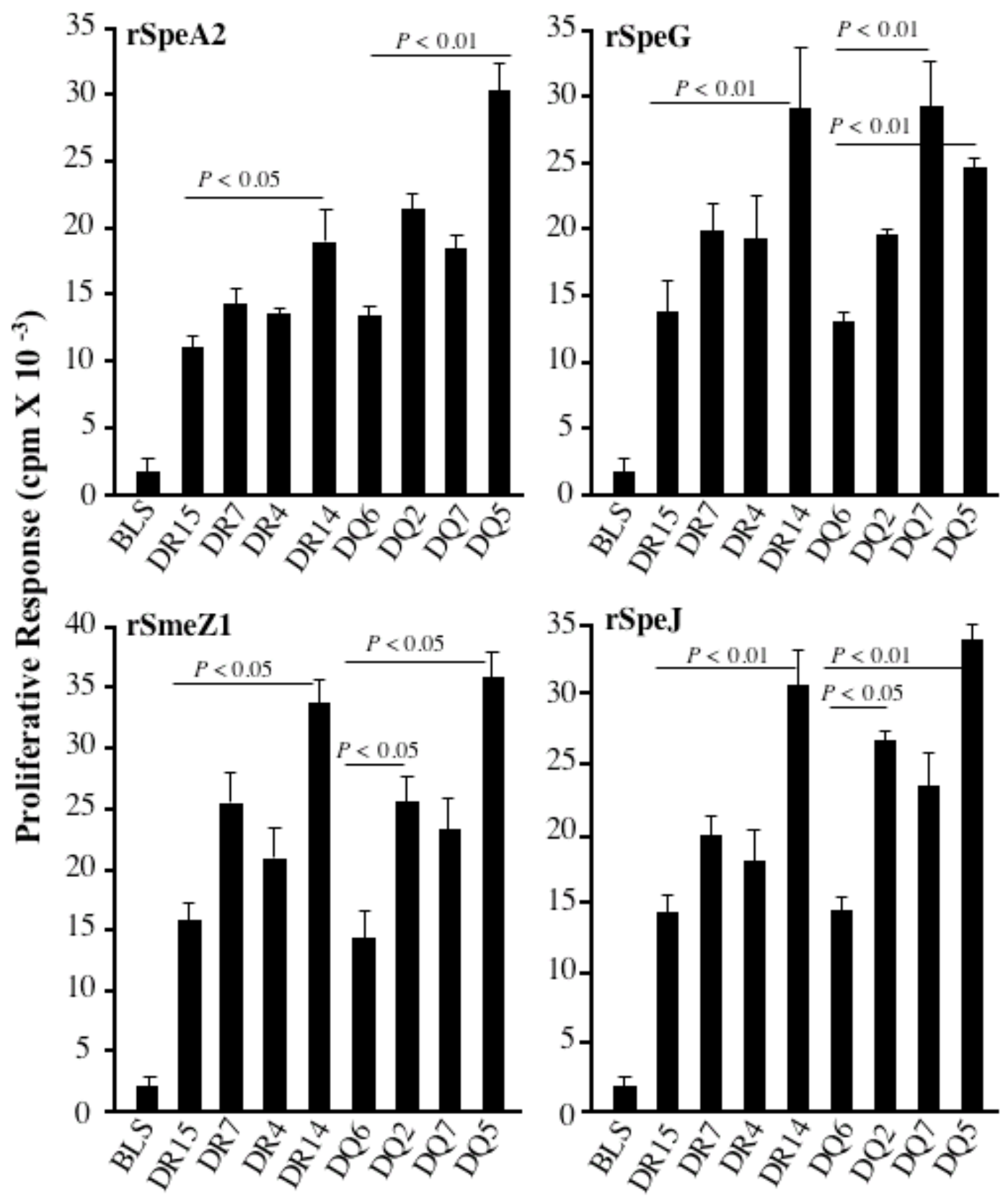

Figure 4.3 Proliferative response to individual M1T1 GAS rSAgs presented by single class II alleles.

Pure T cells from healthy volunteers were stimulated with the individual rSAg: SpeA2, SpeG, SpeJ and SmeZ1 (10 ng/ml) or PHA (1 ug/ml) presented by untransduced BLS cells or transduced with the indicated HLA DR or DQ alleles. The experiments were repeated at least 3 times and the data presented are mean proliferative responses $\pm \mathrm{SE}$, after normalization for any variability in HLA expression level. Significance was evaluated by ANOVA. 
whereas the HLA DR15 was consistently associated with lowest responses compared to high risk or even neutral alleles. These data are in perfect agreement with our epidemiologic data, where we found that both HLA DR14 and DR7 are associated with high risk for STSS, whereas HLA DR15 conferred strong protection from it (275). Similar observations held true for the HLA DQ alleles. Presentation of all tested rSAgs by the HLA DQ6 allele consistently elicited the lowest response, while presentation by the HLA DQ5, which is part of the high-risk haplotype, consistently elicited the highest response with one exception. The presentation of rSpeG by HLA DQ7 resulted in higher responses than by HLA DQ5. This latter finding support previous reports showing that certain SAgs may interact with different HLA class II alleles in a unique fashion (288). Also, in agreement of our earlier studies, the overall presentation of SpeA2 by HLA DQ alleles was relatively superior to that by HLA DR alleles (288). As expected, the class IInegative, BLS cells failed to promote SAg-induced proliferation. These experiments demonstrate that differences in the presentation of Strep SAgs are not related to differences in APC (BLCL or transduced BLS cells), but rather depend on the APCexpressed class II allele and on the interacting SAg. Furthermore, the results suggest that single alleles, which are part of haplotypes associated with protection or susceptibility to STSS, can present SAgs in a manner analogous to these original haplotypes and hence individuals with such alleles may be protected or predisposed to SSD apart from being homozygous or heterozygous.

Cytokine responses were also assessed in cultures containing rSmeZ1 presented by HLA DR14 versus DR15 and HLA DQ5 versus DQ6. In agreement with the proliferative results, presentation of rSmeZ1 by the high-risk HLA DR14 or DQ5 alleles 
induced significantly higher levels of the pro-inflammatory cytokines IL-2, IL-6, TNF- $\alpha$, TNF- $\beta$, and IFN- $\gamma$ than presentation by the protective HLA DR15 or DQ6 alleles, respectively ( $\mathrm{p}<0.001-0.05$; Fig 4.4). However, significantly higher levels of the antiinflammatory cytokine IL-10 were induced by HLA DR 15 or DQ6 alleles than by HLA DR14 or DQ5 alleles ( $>0.01-0.05$, Fig 4.4). These results are in agreement with our previous finding of higher IL-10 production by PBMC from individuals carrying protective alleles or by BLCL homozygous for the protective haplotype DR15/DQ6 and further support our hypothesis that protective haplotype/alleles may mediate their effects partly through active suppression of SAg-induced immune response by promoting high levels of the immunosuppressive cytokine IL-10.

\section{Binding affinity of rSpeG, rSpeJ and rSmeZ1 to different HLA class II alleles}

Although, co-stimulatory molecules on both T cells and APC are involved in T cell activation by SAgs, binding to HLA class II plays a major role in this process. Therefore, we investigated whether differences in the presentation of rSAgs were due to variations in class II alleles binding affinity. Our binding studies demonstrated a correlation between the observed preferential SAg presentation by HLA DR or DQ alleles and binding affinity, where cells expressing high-risk alleles DR14 or DQ5 showed significantly higher SAg binding than cells expressing protective alleles DR15 or DQ6 for all of the tested SAgs (Fig. 4.5A\&B), except for SmeZ1 (Fig. 4.5C). Binding of rSmeZ1 to DQ alleles inversely correlated with the proliferative responses such that HLA DQ6-expressing cells showed significantly higher rSmeZ1 binding than HLA DQ5expressing cells. This suggests that although SmeZ1 binds more avidly to DQ6 

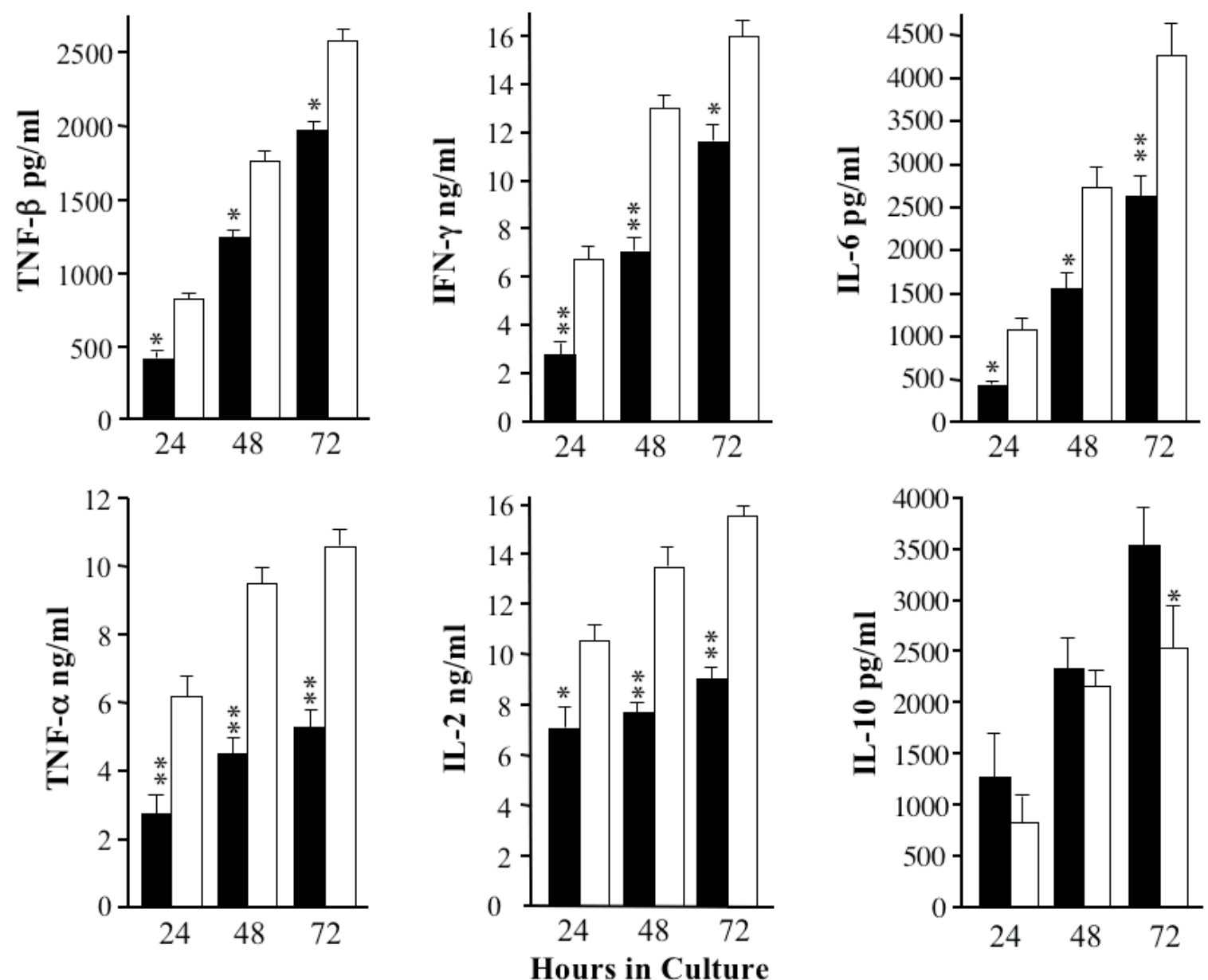

Figure 4.4 Cytokine responses to SmeZ1 presented by the indicated HLA DR or DQ alleles.

The cultures and stimulation were set up as indicated in Figure 4.3 legend. At the specified times points the culture supernatants were collected from cultures containing: $A$. HLA DQ5 or DQ6 and $B$. HLA DR14 or DR15. Supernatants were analyzed for the production of IL-2, IL-6, IL-10, TNF- $\alpha$, TNF- $\beta$ and IFN- $\gamma$. The experiments were repeated at least three times, and the presented data are means \pm SE. Statistical significance was assessed by ANOVA $(* * \mathrm{P}<0.01, * \mathrm{p}<0.05)$. 
B
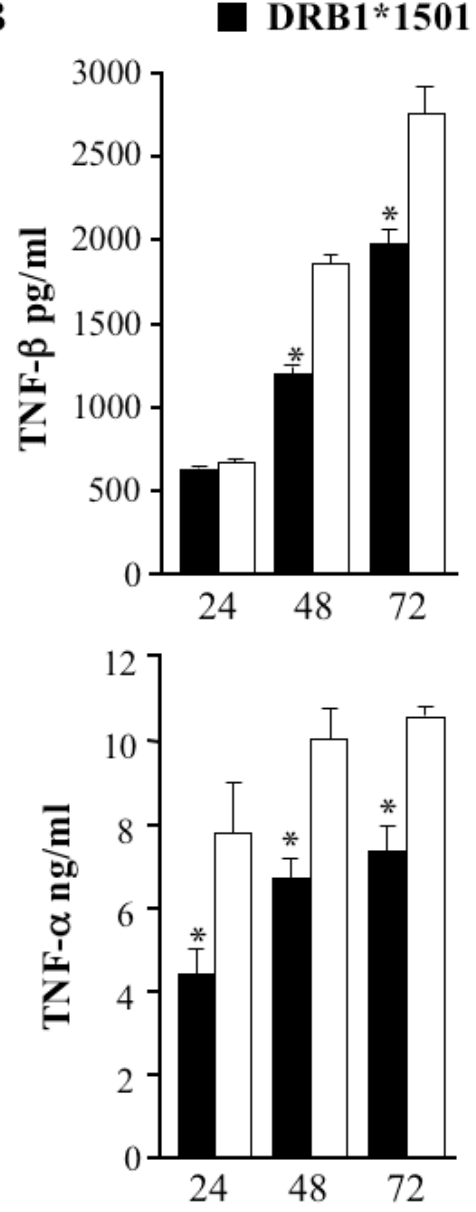

DRB1*1401 (High Risk)
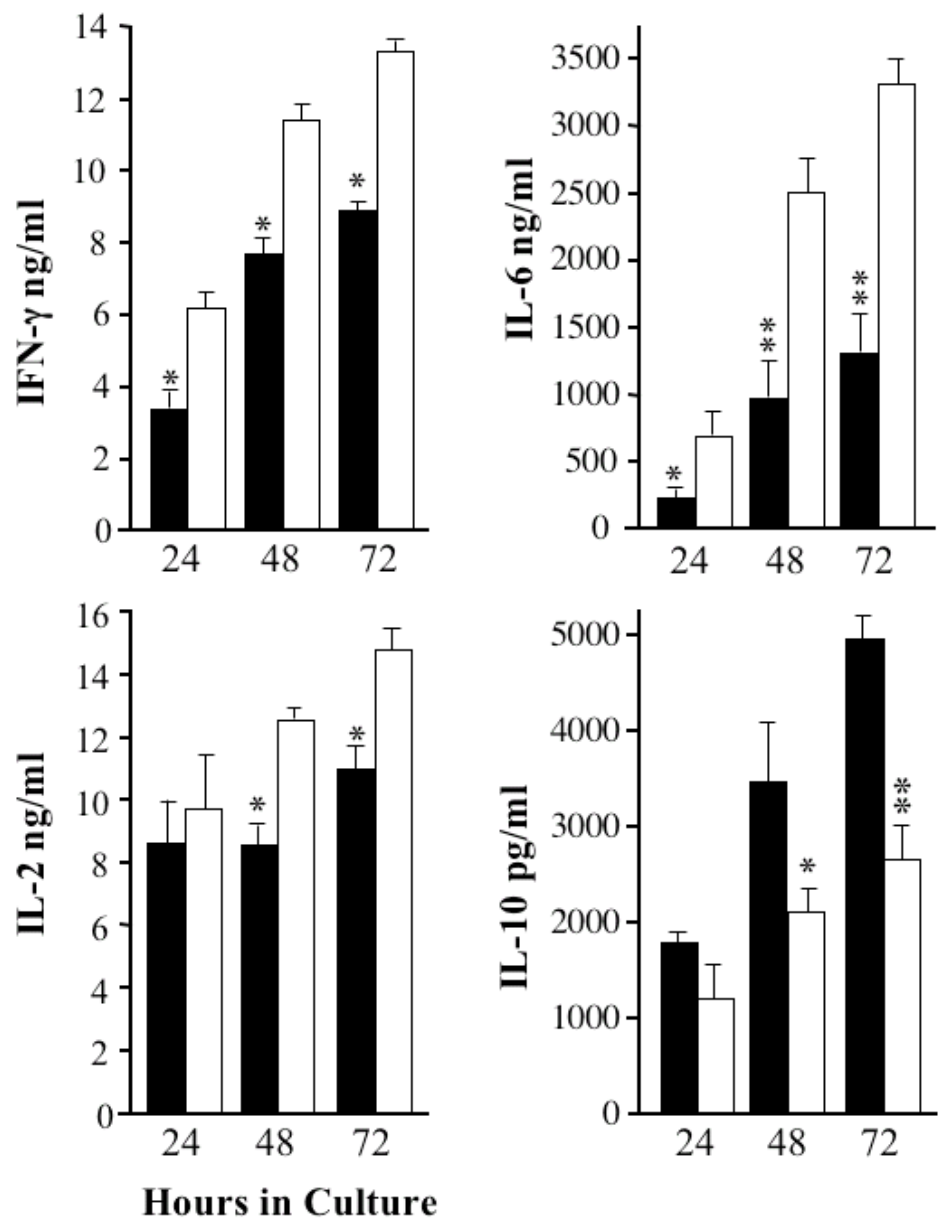

Figure 4.4 (continued). 


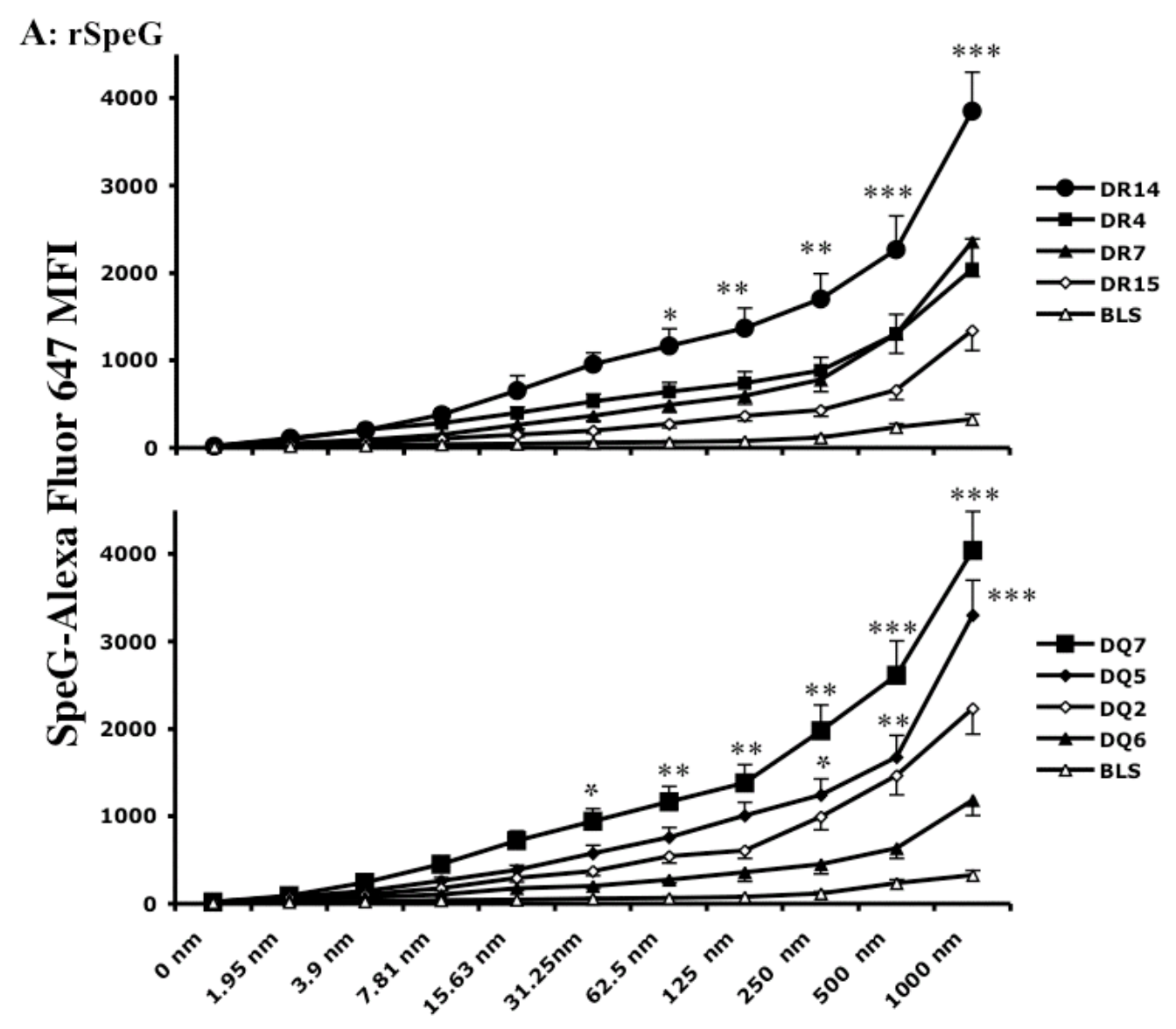

Figure 4.5 Differential binding of SpeG, SpeJ or SmeZ1 by distinct HLA class II alleles.

BLS cells $\left(10^{6} /\right.$ tube $)$ expressing the indicated alleles were incubated with serial dilutions of Alexa fluor 647-labeled: A. SpeG, B. SpeJ or C. SmeZ1. After washing, cells with bound SAg were analyzed by flow cytometry and expressed as mean fluorescence intensity (MFI). Shown is a representative of three independent experiments. Statistical significance was assessed by ANOVA $\left(* * * \mathrm{P}<0.001,{ }^{*} \mathrm{P}<0.01, * \mathrm{p}<0.05\right)$. 

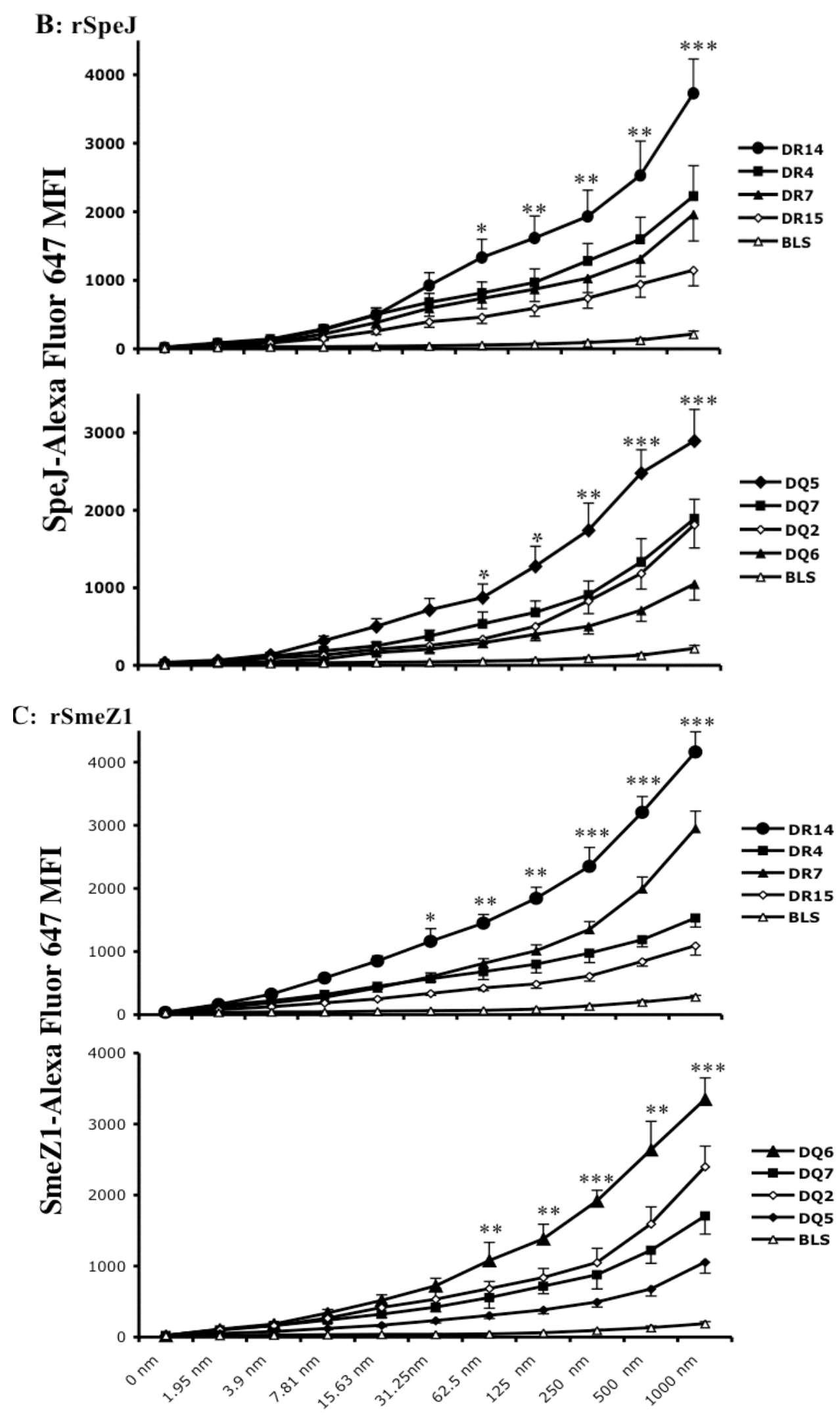

Figure 4.5 (continued). 
but it binds in a manner that does not allow T cell stimulation. Moreover, cells expressing neutral alleles demonstrated intermediate SAg binding with the exception of rSpeG, which in agreement with the observed proliferation results that exhibited higher binding to DQ7- than to DQ5-expressing cells. These observations support the view that each SAg interacts distinctively with HLA DR or DQ alleles. Additionally, differential binding affinity of SAgs to distinct class II alleles cannot always be the underlying mechanism for the observed preferential presentation.

\section{Changes in $\left[\mathrm{Ca}^{2+}\right]_{\mathrm{i}}$ levels in response to stimulation with rSmeZ1}

To this end, we have examined SAg-driven responses at the initial stages (binding to class II molecules on APC) as well as the final steps (T cell proliferation). However, SmeZ1 contradicting behavior in the context of HLA DQ alleles (binding inversely correlated with proliferative responses) prompted us to investigate SmeZ1-induced changes in intracellular calcium level $\left[\mathrm{Ca}^{2+}\right]_{\mathrm{i}}$, an important signaling intermediate in the SAg-driven $\mathrm{T}$ cell activation that may provide novel insight into preferential SAg presentation. As expected, SmeZ1 presentation by DR15 allele had the lowest effect on the magnitude of the $\mathrm{Ca}^{2+}$ response (Fig. 4.6). Interestingly, SmeZ1 presentation by HLA DQ6 induced the weakest $\mathrm{Ca}^{2+}$ response. Therefore, changes in kinetics of $\mathrm{T}$ cells $\mathrm{Ca}^{2+}$ mobilization may be influenced not only by differences in SAg-class II binding affinity but also by the stability of the trimolecular complex of TCR-SAg- HLA class II. Possibly, the presence of a high affinity TCR could stabilize an apparently weak interaction between SmeZ1 and HLA DQ5 molecules, and the formation of such ternary complex would lead to more efficient $\mathrm{T}$ cell activation. 


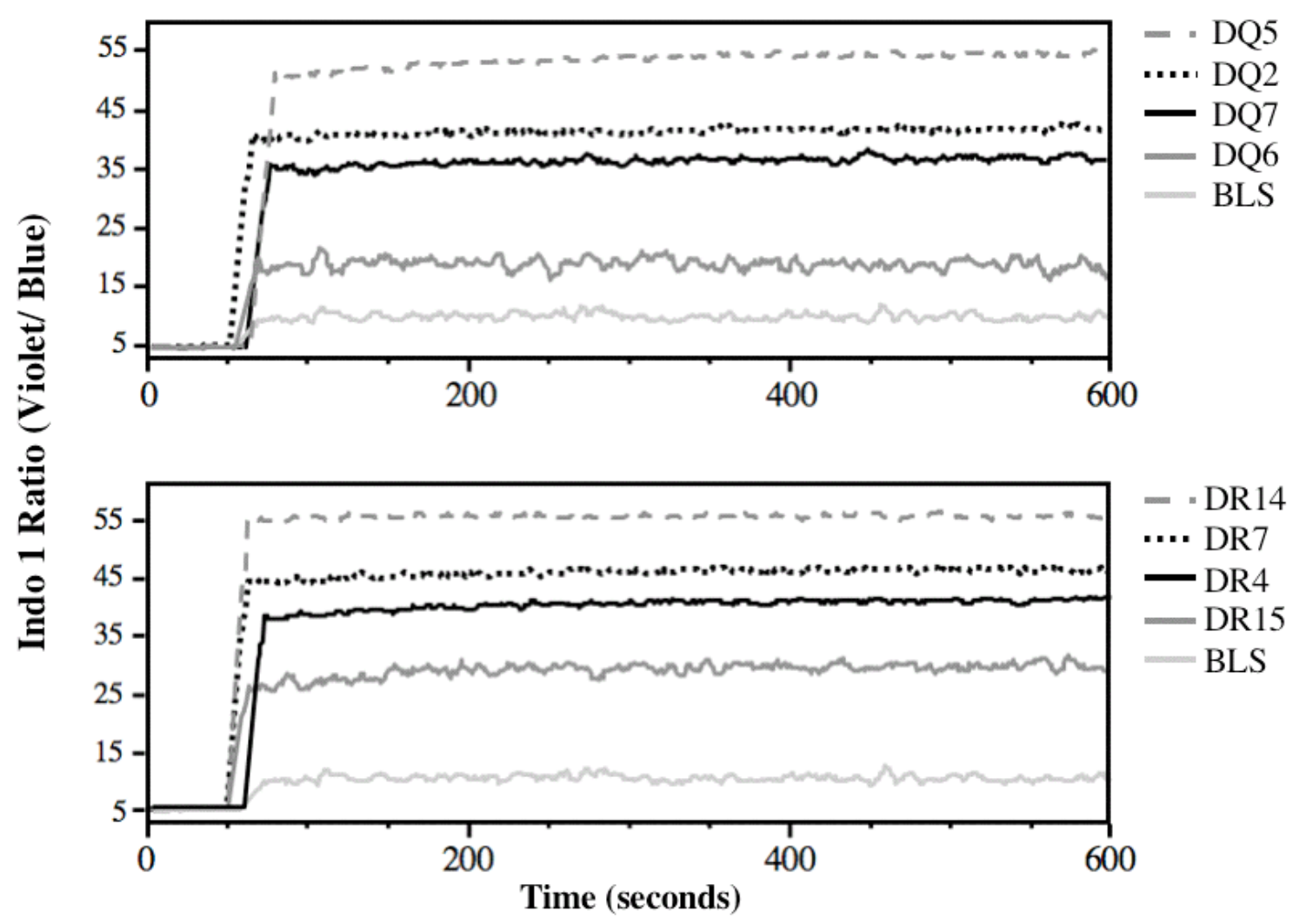

Figure 4.6 Calcium mobilization studies.

BLS cells $\left(10^{6} / \mathrm{ml}\right)$ expressing the indicated HLA DR or DQ alleles were loaded with SmeZ1 $(0.5 \mathrm{ug} / \mathrm{ml})$ and incubated overnight at $37^{\circ} \mathrm{C}$. Indo I-loaded T cells $\left(10^{6} /\right.$ tube $)$ were used to establish a baseline before stimulation with SmeZ1-loaded APC. The data are representative of three independent experiments. 


\section{Discussion}

HLA class II molecules function as receptors for SAgs; therefore interaction with these molecules is a primary step in SAg-mediated responses $(100,354)$. Several studies have shown that allelic variations in HLA class II can influence SAgs presentation (288, 289). Accordingly, HLA class II polymorphisms can govern the extent of SAg-driven inflammatory immune response and subsequent immunopathology and ultimately influence the outcome of SAg-induced illness $(275,276)$. A deeper understanding of this process can help us figure out the reasons for diverse manifestations of SAg-mediated diseases in different individuals and develop effective treatment policies.

Our previous studies indicated a direct and dominant influence of HLA class II allelic variation on the magnitude of cytokine and proliferative responses induced by Strep SAgs $(275,276,288,319)$ (Chapter 4). However, these studies were narrow in terms of the limited class II haplotypes/alleles investigated. Thus, in the present study we utilized a panel of eleven BLCL homozygous for class II haplotypes prevalent in places with high STSS incidence. The application of this panel allowed us to simultaneously study the relative abilities of several distinct HLA class II haplotypes to present Strep SAgs. The data presented here indicate that individual or mixed M1T1 SAgs are preferentially presented by class II haplotypes associated with STSS high-risk DR14/DQ5 or DR7/DQ2 or even by neutral haplotypes DR4/DQ8.

Since these BLCL express all class II isotypes DR, DQ and DP, they are unsuitable for in depth studies to further dissect SAgs-driven responses at the level of single class II allele, to delineate the contribution of polymorphism of individual alleles to variable SAgs responses and to perform biochemical studies with more defined system 
with less variables. To attain this objective we generated a panel of eight BLS lines expressing distinct HLA DR (four) or DQ (four) alleles (Table 3). By using this panel of APC, we found that individual alleles, which are part of STSS-protective or high risk haplotypes, presented individual M1T1 SAgs in a manner equivalent to that of the original haplotypes.

Our previous studies showed that HLA class II association with NF are distinct from that with STSS (275). This observation suggested that STSS and NF are different manifestations of Strep SSD. In agreement with this view, NF-high risk haplotype/alleles reproducibly promoted lower SAg responses than NF-protective haplotype/alleles. This finding suggests that NF might be a SAg-independent manifesataion and thus may require distinct therapeutic approaches.

SAgs have highly variable primary amino acid sequence; however, solved crystal structures for many staphylococcal and Strep SAgs show that they share a conserved three-dimensional structure with two domains, a C-terminal $\beta$-grasp motif and an $\mathrm{N}$ terminal $\beta$-barrel domain $(103,354)$. Such conserved folding may explain the consistently high or low responses to individual M1T1 SAgs in the context of the highrisk or protective HLA class II alleles, respectively. However, solved or computergenerated SAg-HLA class II complex structures have indicated that SAgs bind HLA class II in distinct ways that may affect SAg responses. Some SAgs bind the $\alpha$-chain through a low affinity $\mathrm{N}$-terminal binding site, others bind the $\beta$-chain by high affinity $\mathrm{C}$-terminal zinc ion coordinated binding site and a third group bind both chain and thus crosslink class II molecules on APC and induce more potent responses $(103,150)$. Despite these variable ways of interaction with HLA class II molecules that affect SAg potency, all of 
the studied M1T1 SAgs bind to the $\beta$ chain with the exception of SpeA2, which binds exclusively to the $\alpha$-chain $(106,150)$. Even, this difference in class II binding did not affect the consistent high response induced by high-risk alleles.

The most logical explanation for the superior SAg responses in the presence of high-risk alleles is the enhanced binding of SAgs to such alleles and therefore an increased ligand concentration available for presentation to $\mathrm{T}$ cells. The binding results for rSpeG, rSpeJ and rSmeZ1 supported this hypothesis. However, in case of rSmeZ1, there was a lack of correlation between the ability of HLA DQ5 to bind SmeZ1 and the ability of SmeZ1-HLA DQ5 complex to stimulate T cells. This result may suggest that T cell stimulation under these conditions depends primarily on the formation of a ternary complex of TCR-SAg-HLA class II, with the stability of HLA DQ5-SmeZ1-TCR complex being greater than that of HLA DQ6-Smez1-TCR complex. A TCR with stronger affinity for SAg-HLA may be able to stabilize ineffective interaction between SAg and HLA molecule and thus T cells can react to a particular SAg-HLA combination, even though the last cannot easily be revealed by simple binding studies.

As we and others found, binding affinity of a specific SAg to a given HLA class II allele did not always correlate with the ability of that class II allele to present the SAg $(284,288,289)$. In an attempt to explain the observed disagreement between SmeZ1 binding to and presentation by HLA DQ alleles, we examined $\mathrm{T}$ cells $\mathrm{Ca}^{2+}$ influx as one of the early signals initiated following engagement of the $\mathrm{T}$ cell receptor. $\mathrm{Ca}^{2+}$ serves as a general second messenger in many cells including immune system cells such as T and B cells, monocytes and macrophages. In these cells, $\mathrm{Ca}^{2+}$ plays a crucial role in activation, effector functions, gene expression or differentiation $(355,356)$. Qualities of the 
generated $\mathrm{Ca}^{2+}$ signal such as strength and duration may shape $\mathrm{T}$ cell responses to activation $(357,358)$. Interestingly, our results show that SmeZ1 presentation by DQ5 (associated with STSS high-risk, poor SmeZ1 binding but more efficient presentation) induced about three fold more $\mathrm{Ca}^{2+}$ influx than presentation by DQ6 (associated with STSS protection, stronger SmeZ1 binding, but inefficient presentation). These results suggest that the molecular requirements and biochemical pathways leading to $\mathrm{T}$ cell activation may vary depending the nature of the SAg and the type of HLA class II molecule and $\mathrm{T}$ cells.

The results obtained from this study may promote more thorough understanding of the effects of HLA class II allelism on the SAg-driven responses and better predicting the outcome of SAg-associated disease in a given patient. Possibly, treatment strategies can be customized depending on HLA class II haplotype. Similar to other clinical conditions, specific interventions can produce more effective results than general therapeutic approaches. 


\section{Chapter 5. Streptococcal Mitogenic Exotoxin Z, SmeZ, Is the M1T1 Streptococcal Superantigen Most Susceptible to Degradation by the Streptococcal Cysteine Protease, $\mathrm{SpBB}^{*}$}

\section{Introduction}

Group A streptococci (GAS) are Gram positive bacteria responsible for human diseases with varying severity $(9,100)$. The outcome of these infections is affected by a complex interplay between several surface-associated and secreted bacterial virulence factors, and host immune defenses $(275,318)$. Streptococcal pyrogenic exotoxins (Spes) are well known for their 'super'-antigenic activity and have been shown to be pivotal in the pathogenesis of streptococcal toxic shock syndrome (STSS) and necrotizing fasciitis (NF, the flesh eating disease) $(101,102)$. SAgs are unusual bacterial toxins that play an important role in the pathogenesis of several diseases including food poisoning, autoimmunity, and toxic shock syndrome (TSS) $(100,349)$. A major feature of SAgs is that they bind directly to human leukocyte antigen (HLA)-class II molecules and to the Tlymphocyte receptor (TCR) causing the activation of larger numbers of T-lymphocytes, almost 1000-fold more than those activated by regular antigens (100). This unconventional mode of activation causes excessive production of pro-inflammatory cytokines that can lead to disseminated intravascular coagulation, shock, and multiple organ system failure $(100,214,349)$. While all GAS isolates examined thus far secrete

\footnotetext{
* Adapted by permission of American Society for Biochemistry and Molecular Biology from Nooh MM, Aziz RK, Kotb M, Eroshkin A, Chaung WJ, Proft T, and Kansal R. (2006). Streptococcal Mitogenic Exotoxin, SmeZ, is the Most Susceptible M1T1 Streptococcal Superantigen to Degradation by the Streptococcal Cysteine Protease, SpeB. J Biol Chem 281, 35281-35288.
} 
one or more SAg, different strains can produce very different spectra of the $>24$ streptococcal SAgs. One of the most clinically relevant strains, the M1T1 strain, whose appearance coincided with the resurgence of severe streptococcal infections around the world in the 1980s, has the following SAg-encoding genes: speA2, speF, speG, speJ, and SmeZ1 (274). SpeA2, a horizontally acquired SAg, that has been recently introduced into this strain, has been shown to be a major player in the pathogenesis of STSS. Another streptococcal superantigen, SmeZ1, was shown to play an important role in streptococcal pathogenesis $(226,359)$.

M1T1 isolates obtained from patients with invasive GAS disease of varying severity carry identical spe genotypes but may differ in their SAg expression phenotype (274). Whereas the disease severity could not be correlated to a particular M1T1 SAg, there was a highly significant inverse correlation between severity of the invasive infection and the expression of the streptococcal cysteine protease, which is an important GAS virulence factor. SpeB modifies host proteins like matrix proteins thereby facilitating bacterial invasion, and it can post-translationally cleave and activate precursors for several cytokines and important components of the coagulation pathway $(360,361)$. However, SpeB can also modify and/or inactivate several important GAS virulence proteins, including the anti-phagocytic M protein, the anti-chemotactic C5a peptidase as well as many secreted virulence proteins (360-363). The activity of SpeB seems to be specific as certain bacterial proteins resist its proteolytic activity (363). We demonstrated that the expression of SpeB significantly reduces the immunestimulating activity of secreted native M1T1 SAgs (363). However, we never saw complete loss of the superantigenic activity, even in the presence of high concentrations 
of secreted or added rSpeB. The partial loss of proliferative activity and the fact that the SAgs responsible for the expansion of SmeZ1 specific T cells with TCRV $\beta 4$, TCRV $\beta 7$, and TCRV $\beta 8$ was blocked in the presence of SpeB while the expansion of T cells expressing TCRV $\beta 2$ was unaffected, suggested that SpeB selectively degrade some but not all SAgs produced by M1T1 GAS.

The present study was designed to examine the effect of SpeB on the streptococcal SAgs individually or in combination, as they exist in their native form during infection. We demonstrate a significantly higher susceptibility of SmeZ1 to SpeBproteolysis that was correlated to the presence of an exposed loop on the surface of this protein, as revealed by bioinformatics and structural analysis.

\section{Materials and Methods}

\section{Generation of recombinant GAS superantigens}

rSpeA2, rSmeZ1, rSpeG and rSpeJ were expressed as histidine-tagged fusion proteins containing a factor Xa protease site according to the manufacturers' recommendations (Promega, Madison, WI. and Qiagen Inc.) Primer pairs used for the cloning were as follows: SpeA2 (672bp-5' gaggcctcaacaagaccccgatc 3' forward and 5'gaagcttacttggttgttaggtagact3' reverse), SmeZ1 (645bp-5'gaggcctttagaagtagataataattc3' forward and 5'caaagcttaggagtcaatttc3' reverse), SpeG (630bp-5' taggcctgatgaaaatttaaaag3' forward and 5' caagcttctagtgcgtttttaa3' reverse) and SpeJ (630bp-5' gaggcctatgaaaagaataataaaaacaa 3' forward and 5' gaagcttatttagtccaaaggtaa 3' reverse). The forward and reverse primers contained Stuland HindIII restriction sites, 
respectively. The amplified products were purified, subcloned in pGEM-TEasy vector (Promega Corporation, Madison, WI, USA), and sequenced (UTHSC Molecular Resource Center). The correct cloned inserts were digested with Stul and HindIII, subcloned into the pQE30Xa vector (Qiagen Inc.), propagated in DH5 $\alpha$ Escherichia coli cells and subsequently used to transform M15 Escherichia coli cells. rSAg protein expression was induced with $1 \mathrm{mM}$ IPTG (isopropyl-b-D-thiogalactopyranoside) for $3 \mathrm{~h}$. Proteins in the bactrerial cell lysate were resolved by SDS polyacrylamide gel electrophoresis (PAGE), and the specific expression of each rSAg protein was determined by immunoblotting with RGS-His HRP Conjugate Kit (Qiagen Valenica, CA) and specific rabbit polyclonal anti-Spe antibodies. rSAgs were purified as His fusion proteins using Ni-NTA superflow according to manufacturer's recommendations (Qiagen, Valenica, CA). The purity of rSAgs was confirmed by SDS-PAGE and silver staining. The purified rSAgs were treated with polymyxin B agarose (Boehringer Mannhiem, Indianapolis, IN) to remove any contaminating endotoxin. Enzymatically active rSpeB was generated by one of us (Woei-Jer Chaung) and expressed and purified as detailed earlier (363).

\section{Polyclonal rabbit anti-Spe antibodies}

Specific polyclonal rabbit antibodies to SpeA were generated as previously described (273). Antibodies to SmeZ, SpeG and SpeJ were generated by one of us (T. Proft). 


\section{Effect of SpeB on rSAgs}

Each of the rSAgs $(200 \mu \mathrm{g} / \mathrm{ml})$ was incubated with the pre-activated rSpeB

$(200 \mu \mathrm{g} / \mathrm{ml})$ at $37^{\circ} \mathrm{C}$. We generated the enzymatically active form of rSpeB by incubating the $40-\mathrm{kDa}$ precursor for $8 \mathrm{~h}$ at $37^{\circ} \mathrm{C}$ in the presence of $5 \mathrm{mM}$ dithiothreitol and EDTA. To monitor the kinetics of the effect of rSpeB on rSAgs, we aliquoted samples at $0.5,1$, $2,4,8$, and $18 \mathrm{~h}$ post incubation. We stopped the SpeB enzymatic activity immediately at each time point by adding the cysteine protease inhibitor E-64 (Roche) at a final concentration of $28 \mu \mathrm{M}$. The samples were frozen at $-20^{\circ} \mathrm{C}$ until further analyses. To check the specificity of SpeB's proteolytic activity, we also included a control where its activity was inhibited from the start of incubation by adding E-64. The digestion mixtures $(\mathrm{rSAgs}+\mathrm{rSpeB})$ were separated by SDS-PAGE and transferred onto nitrocellulose paper. We analyzed the proteolytic degradation of the rSAgs following incubation with rSpeB by Western blots using specific anti-Spe antibodies.

\section{Proteomic analysis of the SAg mixture}

The four recombinant M1T1 SAgs (SpeA2, SpeG, SpeJ, and SmeZ1) were mixed in equal amounts $(10 \mu \mathrm{g} / \mathrm{ml})$ and treated with 20 or $200 \mu \mathrm{g} / \mathrm{ml}$ of pre-activated SpeB. The cysteine protease inhibitor, E-64 (final concentration of $28 \mu \mathrm{M}$ ) was used to stop the reaction after $8 \mathrm{~h}$. Prior to isoelectric focusing (IEF), the SAg mixture, with and without active SpeB, was lyophilized to dryness, then resuspended in $125 \mu 1$ rehydration buffer composed of $7 \mathrm{M}$ urea, $2 \mathrm{M}$ thiourea, $4 \%$ CHAPS, $20 \mathrm{mM}$ dithiothreitol and $0.16 \%$ of 40\% Bio-Rad 3-10 ampholytes (Bio-Rad) IEF was performed in a Protean IEF cell, and the second dimension SDS electrophoresis was run on a Protean Minigel Dodeca 
apparatus (Bio-Rad) as detailed elsewhere (362). Protein spots, visualized by silver staining, were cut from the gels, destained, and digested with trypsin. The tryptic peptides were extracted with $60 \%$ acetonitrile/5\% trifluoroacetic acid (TFA), purified on micro-C18 columns (Ziptips-Millipore), and finally eluted in the matrix solution (alphacyano-hydroxycinnamic acid dissolved in 50\% acetonitrile/0.1\% TFA). To identify these proteins, we analyzed their tryptic peptides' masses by MALDI-TOF MS using a Voyager DE RP (ABI) in the Stout Neuroscience Laboratory (UTHSC proteomic facility) and then we used the Aldente software (http://us.expasy.org/tools/aldente/) to match the peptide masses to proteins in the databases.

\section{N-terminal sequencing of the Strep SAg following incubation with rSpeB}

$\mathrm{N}$-terminal protein sequencing (Edman degradation) was performed by Midwest Analytical, Inc. (St Louis).

\section{Bioinformatics analysis of SAg sequence}

We used several bioinformatics tools to analyze the amino acid sequences of the four M1T1 SAgs as well as their secondary and tertiary structures. We aligned the primary sequences using the ClustalW analysis tool (364) in VectorNTI suite (Invitrogen). We used the Consensus Disorder Prediction (CDP) tool from the Center on Proteolytic Pathways (CPP) (http://protease.burnham.org/www/tools/html/disorder.html).

This tool implements five different disorder prediction methods: COILs, REM465, and HOTLOOPs (from disEMBL) and GlobPlot (365) and DISOPRED (366). In CDP, if more than half of these applied methods calculate a residue as disordered, a 
consensus is generated and the residue is scored as disordered. The 3D structures for SpeA and SmeZ were pulled from the Protein Data Bank (PDB) (http://www.rcsb.org/pdb/Wellcome.do) and the Molecular Modeling DataBase (MMDB) (http://www.ncbi.nlm.nih.gov/Structure/MMDB/mmdb.shtm). SpeA accession numbers: 1L0X and 1L0Y, SmeZ accession numbers: 1ET6, 1EU3. The structure models were generated using SWISS-MODEL (367).

\section{Effect of SpeB on the functional activity of streptococcal SAgs}

For assessment of the effect of SpeB on the ability of SAgs to induce lymphocyte proliferation, peripheral blood mononuclear cells $(\mathrm{PBMC})$ were isolated from heparinized blood of healthy individuals as detailed previously (294), and stimulated with each of rSAg with or without pre-incubation with active rSpeB as detailed above. PBMC $\left(10^{5}\right.$ cells/200 $\mu 1$ ) were stimulated with serial two-fold dilutions of either the tested $\operatorname{rSAg} \pm$ rSpeB or the polyclonal mitogen, phytohemagglutinin A (PHA) at $1 \mu \mathrm{g} / \mathrm{ml}$ conc. T cell proliferation was assessed by measuring $\left[{ }^{3} \mathrm{H}\right]$ thymidine uptake after $72 \mathrm{~h}$ of culture.

\section{Analysis of TCR V $\beta$ repertoire by flow cytometry}

Each SAg has a characteristic V $\beta$ specificity and preferentially activates $T$ cells expressing certain $\mathrm{V} \beta$ elements. We used flow cytometry for quantitative analysis of preferential expansion of lymphocytes with specific TCR V $\beta$ s using the IO Test Beta Mark TCR V $\beta$ Repertoire kit (Beckman Coulter; Miami, Florida). We used a CD3-PC5conjugate as an additional marker to enable proper gating on $\mathrm{T}$ lymphocytes only. PBMC were isolated from the blood of a healthy individual by Ficoll-Hypaque gradient 
centrifugation, incubated at $10^{7}$ cells $/ 5 \mathrm{ml}$ of RPMI with $10 \% \mathrm{FCS}$, and stimulated with $5 \mathrm{ng} / \mathrm{ml}$ of each SAg (with or without E-64) or PHA at $1 \mu \mathrm{g} / \mathrm{ml}$. After $72 \mathrm{~h}$, the cells were cultured for additional $24 \mathrm{~h}$ in the presence of $10 \mathrm{units} / \mathrm{ml}$ recombinant human IL-2 to allow the regeneration of modulated $\mathrm{T}$ cell receptors. The cells were then harvested, washed extensively with PBS containing $1 \%$ BSA, and stained with different antibodies as per the manufacturer's instructions. We gated on the CD3-PC5 blastogenic cells and performed simultaneous analysis of 3 TCR V $\beta$ s per tube using a FACSCalibur flow cytometer. We analyzed the data using Cell Quest software (Becton Dickinson, Mountain view, California). A minimum of 30,000 cell events was acquired for the analysis.

\section{Results}

\section{Proteolytic degradation of individual SAgs by SpeB}

The clinically relevant M1T1 strain harbors the genes encoding for SAgs: SpeA2, SpeF, SpeG, SpeH, SpeJ and SmeZ1. Using conventional methods of protein detection and identification, we observed that SpeF is completely degraded in the presence of SpeB (363). Since most of the remaining SAgs viz. SpeJ, SpeG, and SmeZ1 are secreted at levels much below the detection limits of conventional methods $(124,363)$, we extended these studies with the rSAgs.

SpeA2, SpeJ, SpeG, and SmeZ1 appear to be affected differently by the rSpeB (Fig 5.1A). SmeZ1 was most susceptible to proteolytic degradation by SpeB as early as 30 min postincubation and was virtually undetectable after $8 \mathrm{~h}$ even at the lowest SpeB 
Figure 5.1 Differential degradation of individual rSAgs by SpeB.

A. Western blot analysis of the kinetics of M1T1 GAS SAg degradation by enzymatically active $\mathrm{rSpeB}$. Each of the indicated $\mathrm{rSAgs}(200 \mu \mathrm{g} / \mathrm{ml})$ was incubated with equal volume of pre-activated r-SpeB $(200 \mu \mathrm{g} / \mathrm{ml})$. Aliquots $(10 \mu \mathrm{l})$ were removed at $0.5 \mathrm{~h}, 1 \mathrm{~h}, 2 \mathrm{~h}, 4 \mathrm{~h}, 8 \mathrm{~h}$, and $18 \mathrm{~h}$ post incubation and the SpeB enzymatic activity was stopped immediately at each time point by adding the cysteine protease inhibitor E-64 at a final concentration of $28 \mu \mathrm{M}$. Digestion of the rSAg protein was monitored by Western blots using SAg specific antibodies. $B$. SpeB specific degradation of superantigens can be inhibited with E-64. Wetsern blot analysis of His- tagged r-SAgs: SpeA2, SpeJ, SpeG, and SmeZ1 incubated with $200 \mu \mathrm{g} / \mathrm{ml}$ of rSpeB for $8 \mathrm{~h}$ in the absence or presence of E-64. The blots were probed with specific anti Spe antibodies. The bands in the middle lane are moving lower than the rSAgs because rSpeB cleaves $\mathrm{N}$ terminal His tag. 

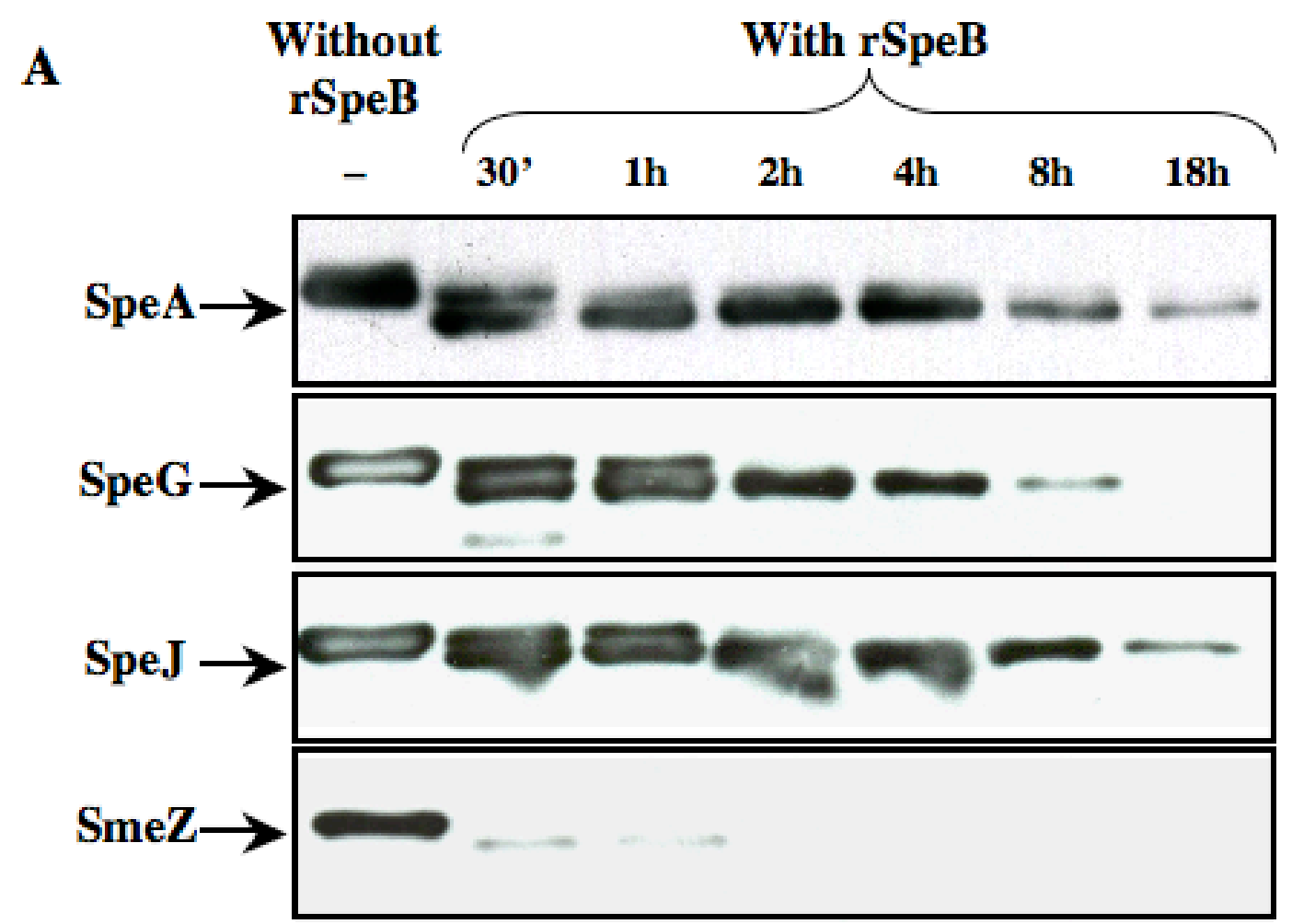

B

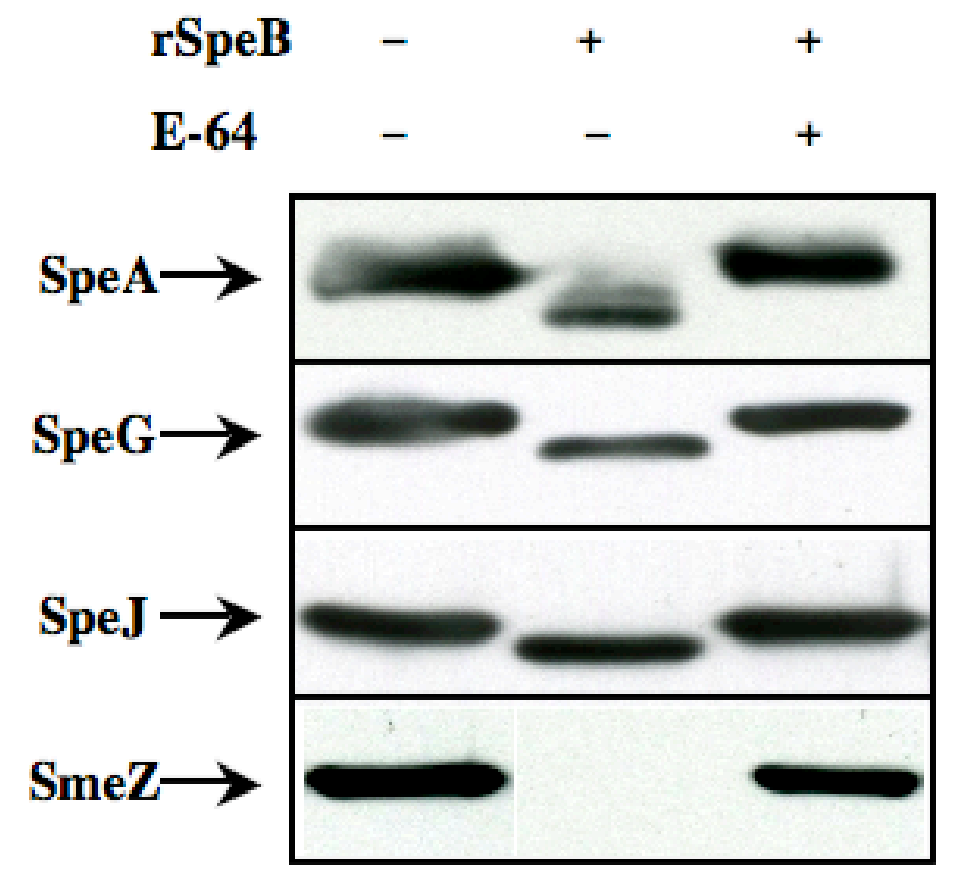


concentration used (Fig 5.2A). Compared to SmeZ1, SpeJ was the most resistant to SpeB degradation even after $18 \mathrm{~h}$ of co-incubation with the highest used dose of SpeB. Whereas SpeJ and SmeZ1 exhibited extreme resistance and susceptibility to SpeB, respectively, the susceptibility of SpeG and SpeA2 to the proteolytic activity of SpeB varied. After $8 \mathrm{~h}$ of incubation, SpeG showed slight and partial degradation by 20 and $200 \mu \mathrm{g} / \mathrm{ml} \mathrm{SpeB}$, whereas partial degradation of SpeA2 was only noted after $18 \mathrm{~h}$ of incubation and only at the highest concentration of the protease (Fig 5.1A \& B). In the presence of E-64, all SAgs were completely protected from SpeB degradation (Fig 5.1B).

Thus far, we have determined the differential cysteine protease activity of rSpeB on each of the four rSAgs individually. In real life, however, these proteins are coexpressed in the GAS secretome. We asked if the presence of a mixture of SAgs would have a synergistic or antagonistic effect on their susceptibility to SpeB. Because these rSAgs have similar molecular weights, we resolved them by two-dimensional gel electrophoresis before and after their incubation with the activated rSpeB. The data shown in Figure 5.2A \& B confirmed the higher susceptibility of SmeZ1 to rSpeB activity and showed that this susceptibility is not altered by the presence of the other three SAgs. The susceptibility of other three SAgs in the mixture showed a similar pattern as that seen with each one of them individually: SpeG showed partial degradation while SpeA2 and SpeJ remained unaffected up to $8 \mathrm{~h}$ of incubation with $\mathrm{rSpeB}$ (Fig 5.2).

\section{SpeB-driven proteolytic degradation of SAgs affects their biological activity}

Following the $8 \mathrm{~h}$ co-incubation with $\mathrm{SpeB}$, the proliferation-inducing activity of SmeZ1 was reduced significantly $(\mathrm{P}=0.003)$ whereas that of $\mathrm{Spe} A 2$ remained unchanged 
Figure 5.2 Reproducible differential degradation of mixed rSAgs by SpeB.

A. All of the indicated $\mathrm{rSAgs}(200 \mu \mathrm{g} / \mathrm{ml})$ were mixed together and incubated for $8 \mathrm{~h}$ with 0,20 or $200 \mu \mathrm{g} / \mathrm{ml}$ of pre-activated $\mathrm{r}$-SpeB. The mixtures were resolved on $12.5 \% \mathrm{SDS}$ polyacrylamide gels. Digestion of the rSAg protein was monitored by Western blots using SAg specific antibodies. B. 2D-gels of mixture of rSAgs (SpeA2:A, SpeG:G, SpeJ:J, SmeZ1:Z) incubated for $8 \mathrm{~h}$ with 0,20 or $200 \mu \mathrm{g} / \mathrm{ml}$ of pre-activated $\mathrm{rSpeB}$ (SpeB:B). The gels were stained with silver (left panels), and the silver stained spots were identified by MALDI-TOF MS. The gels were also blotted onto the nitrocellulose membrane, which were probed with anti-SmeZ1 (middle panels) or anti-SpeA2 (right panels) antibodies as detailed in methods. The lower right panel shows the SpeA2 spot with some tailing; this tailing in 2D gels is due to incomplete isoelectric focusing (also apparent in the silver-stained gel) and may be caused by the excess SpeB (200 $\mu \mathrm{g})$ added in this gel which might have rendered the focusing less efficient than the middle panel. 


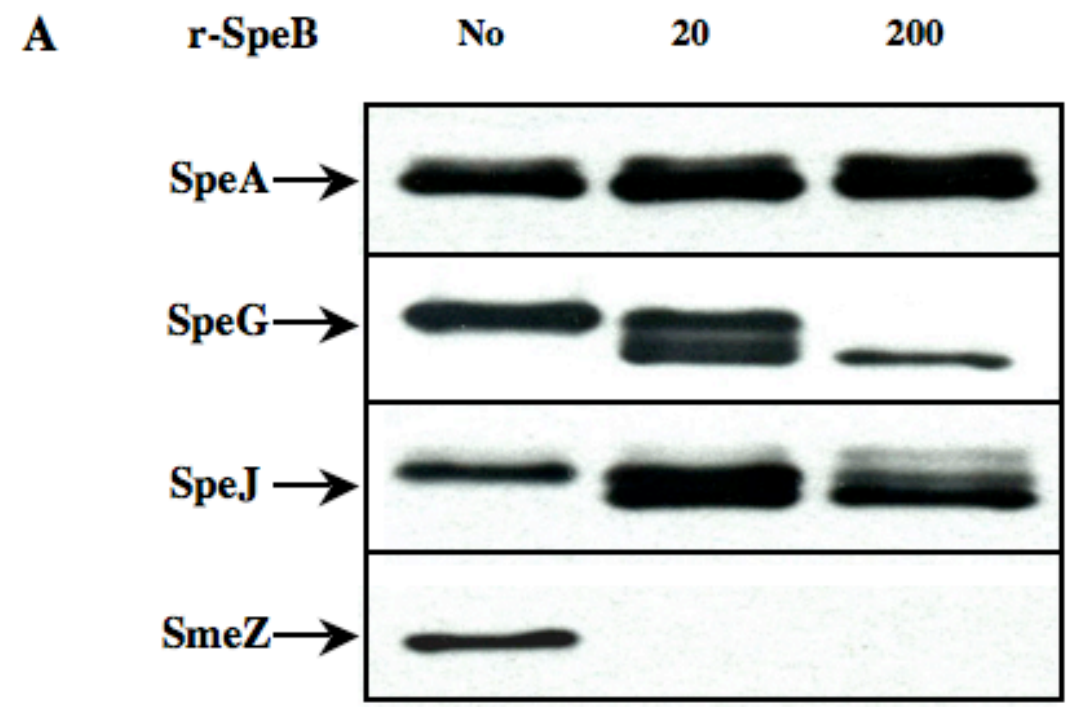

B

rSAg Mix

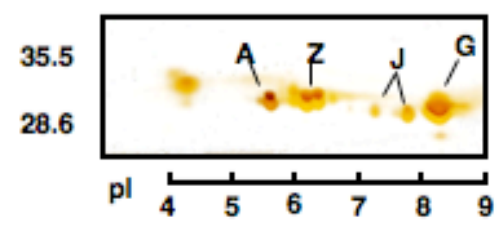

rSAg Mix +rSpeB $(20 \mu \mathrm{g})$

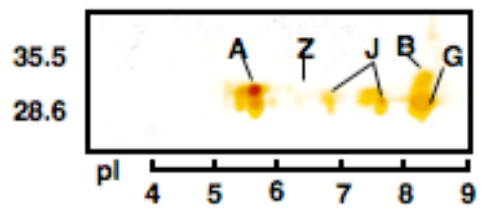

rSAg Mix + rSpeB $(200 \mu \mathrm{g})$

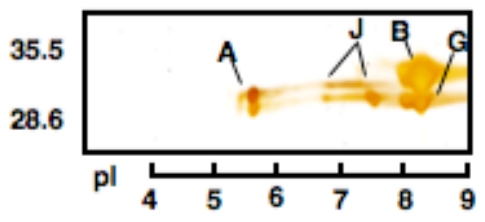

Anti SmeZ
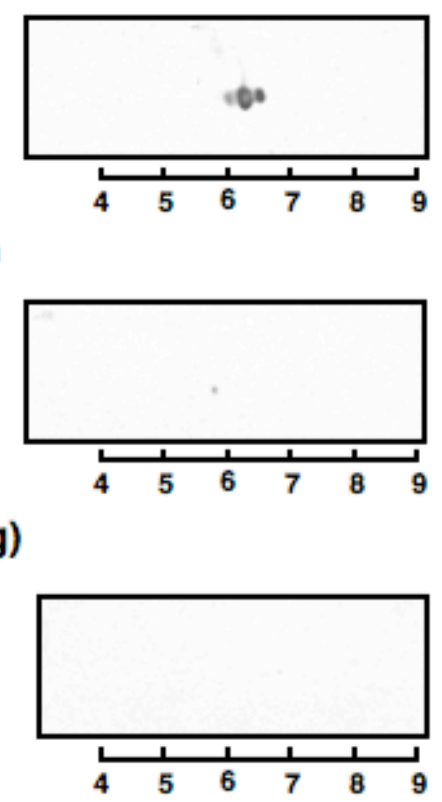

Anti SpeA
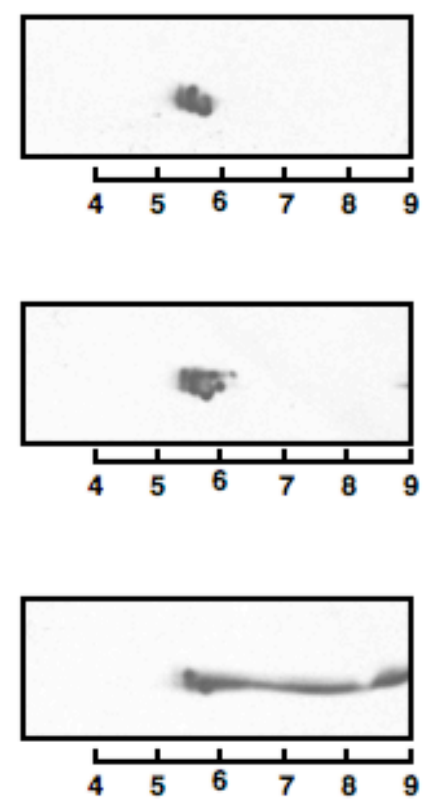
(Fig 5.3A). In fact, the proliferation-inducing activity of SpeA2 following incubation with SpeB appears to have slightly increased and this was seen in several experiments (Fig 5.3A). The loss of SmeZ1 activity following incubation with SpeB could be stopped by the addition of E-64. The proliferation-inducing activity of SpeG and SpeJ was unaffected by SpeB (data not shown).

The proliferation results were supported by TCR V $\beta$ analyses of the human T cells stimulated with a representative SpeB-resistant SAg, SpeA2, or a SpeB-sensitive SAg, SmeZ1. The skewed pattern of TCRV $\beta$ repertoire in response to stimulation with SpeA2 was not altered following pre-incubation with SpeB. By contrast, the ability of SmeZ1 to induce the expansion of $\mathrm{T}$ cells bearing TCRV $\beta 4$, TCRV $\beta 7.1$ and TCRV $\beta 8$, was diminished when the SAg was pre-incubated with SpeB (Fig 5.3B), with a marked reduction in the number of activated $\mathrm{T}$ cells bearing TCRV $\beta 8$ elements.

\section{Specificity of SpeB degradation}

SpeB degraded the His-tag on all the rSAgs used. This was first suggested by the appearance of a slightly faster migrating species in Westerns of SAgs preincubated with active SpeB, including those that resisted its proteolytic effects (Fig 5.1A), and later confirmed when we probed the blots with anti-His antibodies and were unable to detect any SAg bands (data not shown), yet the proteins were readily detected at their expected size by their specific antibodies. These results prompted us to investigate the SpeBspecific cleavage sites on each of these SAgs and to explore why only SmeZ1 is degraded completely while other SAgs are not.

$\mathrm{N}$-terminal sequencing of the protein fragments followed by amino acid alignment 
Figure 5.3 SAg degradation products are not functionally active.

A. T lymphocyte proliferative activity of rSmeZ1 and rSpeA2 incubated without or with $200 \mu \mathrm{g} / \mathrm{ml} \mathrm{rSpeB} \mathrm{(} \pm$ E64) for $8 \mathrm{~h}$. The proliferation inducing activity of each SAg was tested over a wide range (500ng - $5 \mathrm{pg} / \mathrm{ml}$ of the culture media). The results shown here, however, correspond to 2.5 and $10 \mathrm{ng} / \mathrm{ml}$ of SmeZ1 and SpeA2 respectively. The data are representative of 3 independent experiments. Each value represents mean \pm SD of four values. Statistical significance of the differences in the proliferation-inducing responses with or without incubation with rSpeB was determined by Student $t$ test (twotailed).

$B$. SpeB selectively degrades SmeZ1 but spares SpeA2. Expansion profiles of lymphocytes with specific $\mathrm{T}$ cell receptor $\mathrm{V} \beta$ in response to stimulation with SmeZ1 and SpeA2 with or without incubation with $200 \mu \mathrm{g} / \mathrm{ml}$ of $\mathrm{rSpeB}$ for $8 \mathrm{~h}$. The final concentrations used for stimulation of SmeZ1 and SpeA2 were 5 and $10 \mathrm{ng} / \mathrm{ml}$ of the culture media respectively. 
A

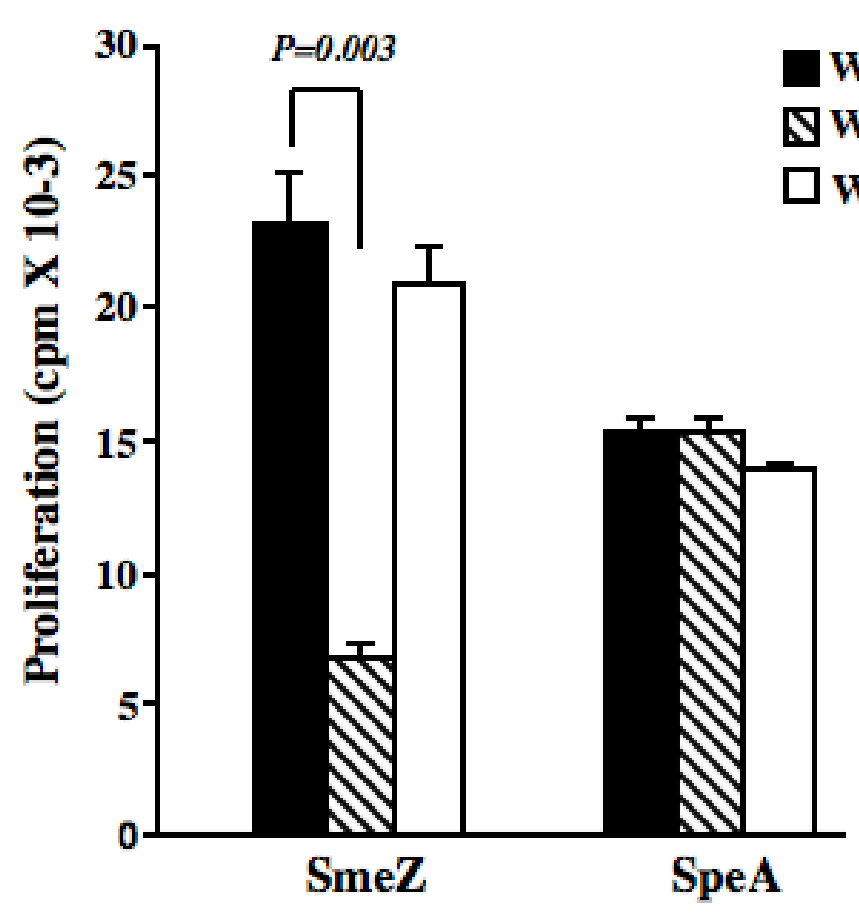

B

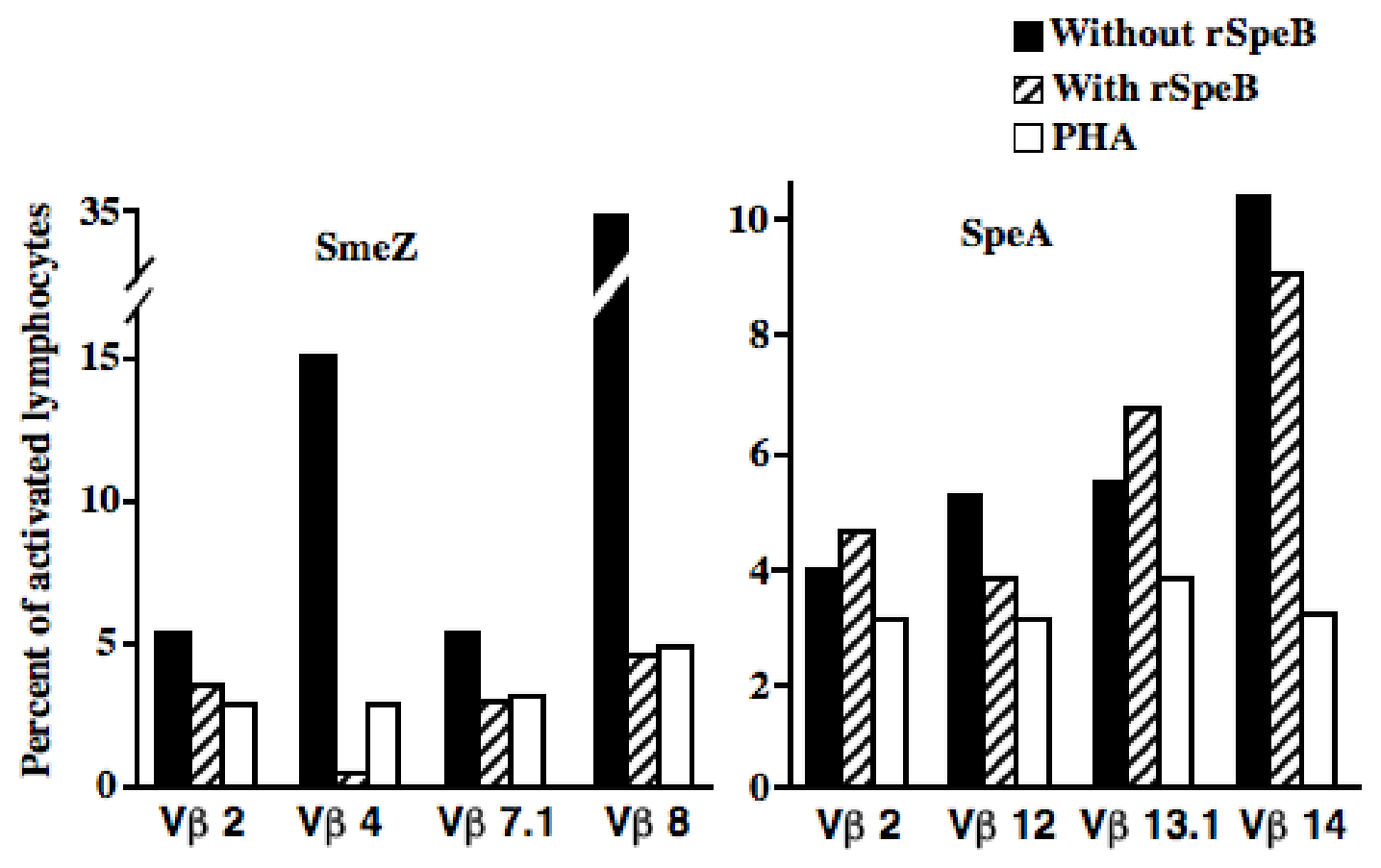


revealed that all four SAgs were cleaved between the glutamate (E) and glycine (G) residues just after the histidine tag (Fig 5.4). These two residues do not belong to the cloned proteins but are rather encoded by the expression vector as part of the factor Xa cleavage site that was engineered between the His-tag and the cloned protein to facilitate the removal of the His-tag. Although SmeZ1 was also initially cleaved at the same site (15 min post incubation with SpeB), it was completely degraded by $30 \mathrm{~min}$.

\section{Bioinformatics analysis reveals a putative SpeB-target loop in SmeZ1}

The consensus target for cleavage by SpeB has been suggested to contain a hydrophobic residue (isoleucine or valine) at the $\mathrm{P}_{2}$ site and a positively charged residue (preferably lysine) at $\mathrm{P}_{1}(368,369)$. Bioinformatics analysis of the primary sequence and the predicted secondary structure of the SAgs used in this study did not reveal a preferential abundance of the SpeB preferred sites in SmeZ1 when compared to the other SAgs. Alternatively, a plausible explanation of why SmeZ1 is totally degraded within an hour while SpeG, SpeA2 and SpeJ are spared could lie in the folds of the tertiary structures of these proteins. Accordingly, it appears that the tertiary structure of SmeZ1 may make some 'SpeB-vulnerable' targets exposed whereas these may be sequestered in other SAgs. Indeed, it was suggested that large local motions proximate to the scissile bonds are required for proteolysis and that the prime determinant for limited proteolysis is this ability to unfold locally (370). Accordingly, we used the CDP method (see experimental procedures) to predict intrinsically disordered regions in the four rSAgs. Subsequently, we looked for the SpeB extended specificity pattern [ILVFMYW]K in regions predicted to be disordered to find potential cut sites of SpeB. 


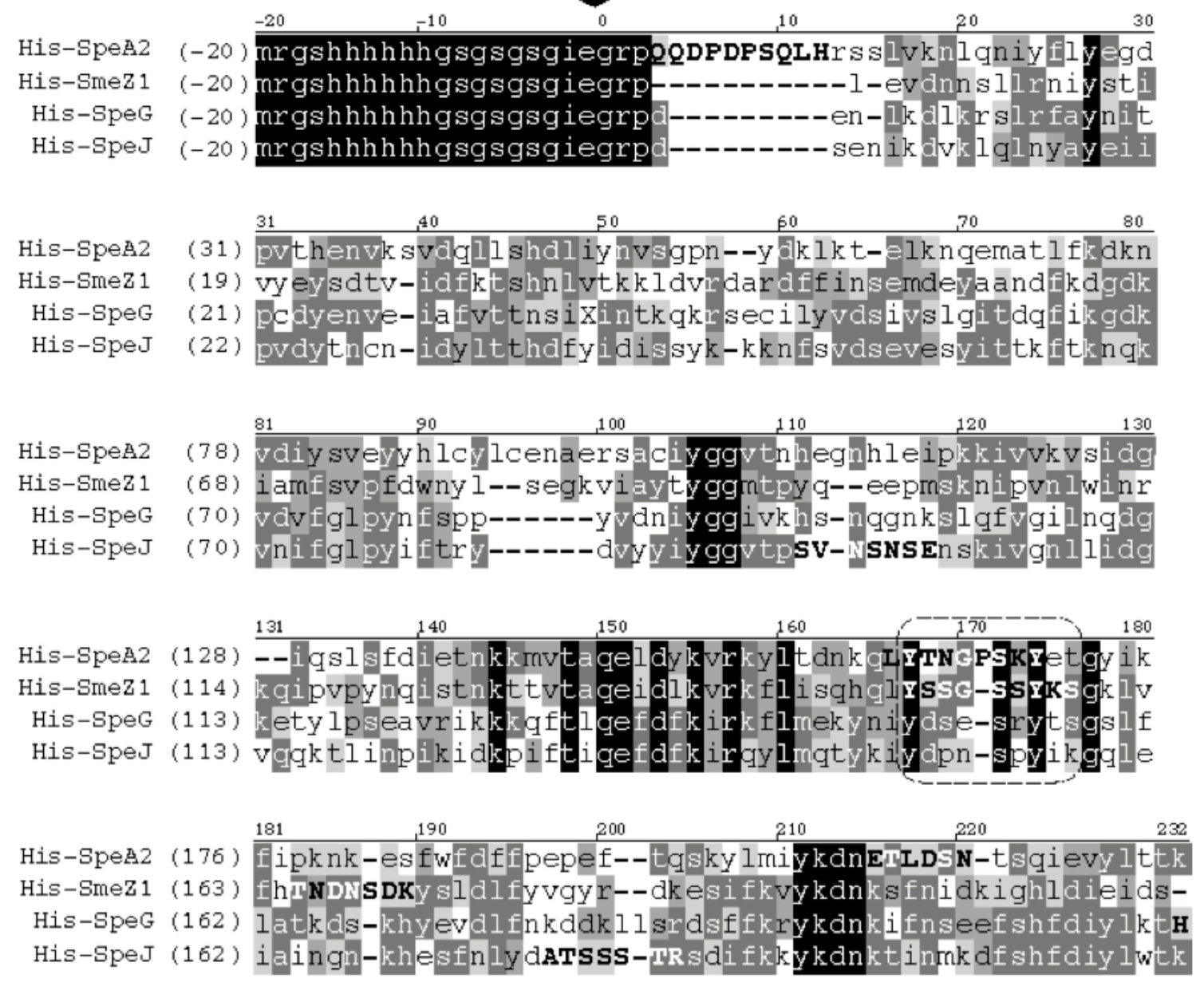

Figure 5.4 Sites of cleavage of the various rSAgs by SpeB.

Alignment of the amino acid sequences of rSpeA2, rSpeG, rSpeJ and rSmeZ1. All of the SAgs studied were cleaved at the N-terminal end of the first $\mathrm{G}$ in the protein (see arrow), thereby removing all extra amino acids added onto the protein as part of the His-tag included in this expression vector. Sequences are aligned by ClustalW tool available in VectorNTI suite. Amino acids likely to be in disordered areas are shown in upper case letters. The flexible loop encompassing the GSSYKS sequence in SmeZ1 (shown in a dashed box) is expected to be the most vulnerable site for SpeB cleavage. In the same loop, note that SpeA2 has an E instead of the K found in SmeZ1 and it is possible that this substitution may have rendered SpeA2 resistant to SpeB. Also, the extra P found in the SpeA2 but not the SmeZ1 loop, may restrict the flexibility of the loop and may contribute as well to its protection from SpeB proteolysis. (SpeA2 and SmeZ1 are the specific alleles of speA and Smez present in the M1T1 GAS). 
The only site in disordered region was found in SmeZ1, boosting the experimental observation of its selective susceptibility to rSpeB. On the other hand, the homologous area in the other SAgs was either not in a disordered region (rSpeG and $\mathrm{rSpeJ}$ ), or was in a disordered region (rSpeA2) but lacking the lysine residue and having an additional proline residue that adds steric hindrance which may interfere with proteolysis (Fig 5.5). Comparing the crystal structures for SpeA2 and SmeZ1 revealed that the areas are surface exposed on loops, with the difference that this loop lacks lysine in SpeA2 (Fig $5.5)$.

\section{Discussion}

SAgs play an important role in the pathogenesis of severe invasive infections caused by Group A Streptococcus $(275,349)$, and their expression is tightly regulated both on the transcriptional and post-translational levels $(28,363,371)$. We reported earlier that SpeB plays a pivotal role in the post-translational degradation of GAS secreted protein, which results in partial loss of proliferation-inducing activity of GAS supernatants containing a mixture of several secreted SAgs (363). In the present study, we demonstrated that SAgs vary considerably in the rate and extent of their proteolytic degradation by SpeB: while some SAgs are highly susceptible, others are resistant to the degradation by SpeB up to 18 hours.

SpeB is a highly conserved GAS protein that, unlike some other phage-encoded streptococcal exotoxins, e.g., SpeA and SpeC, is encoded by chromosomal DNA. SpeB is secreted through the ExPortal, a unique single microdomain of the cellular membrane specialized to contain the Sec translocons (372) as a $40-\mathrm{kDa}$ precursor that can catalyze 

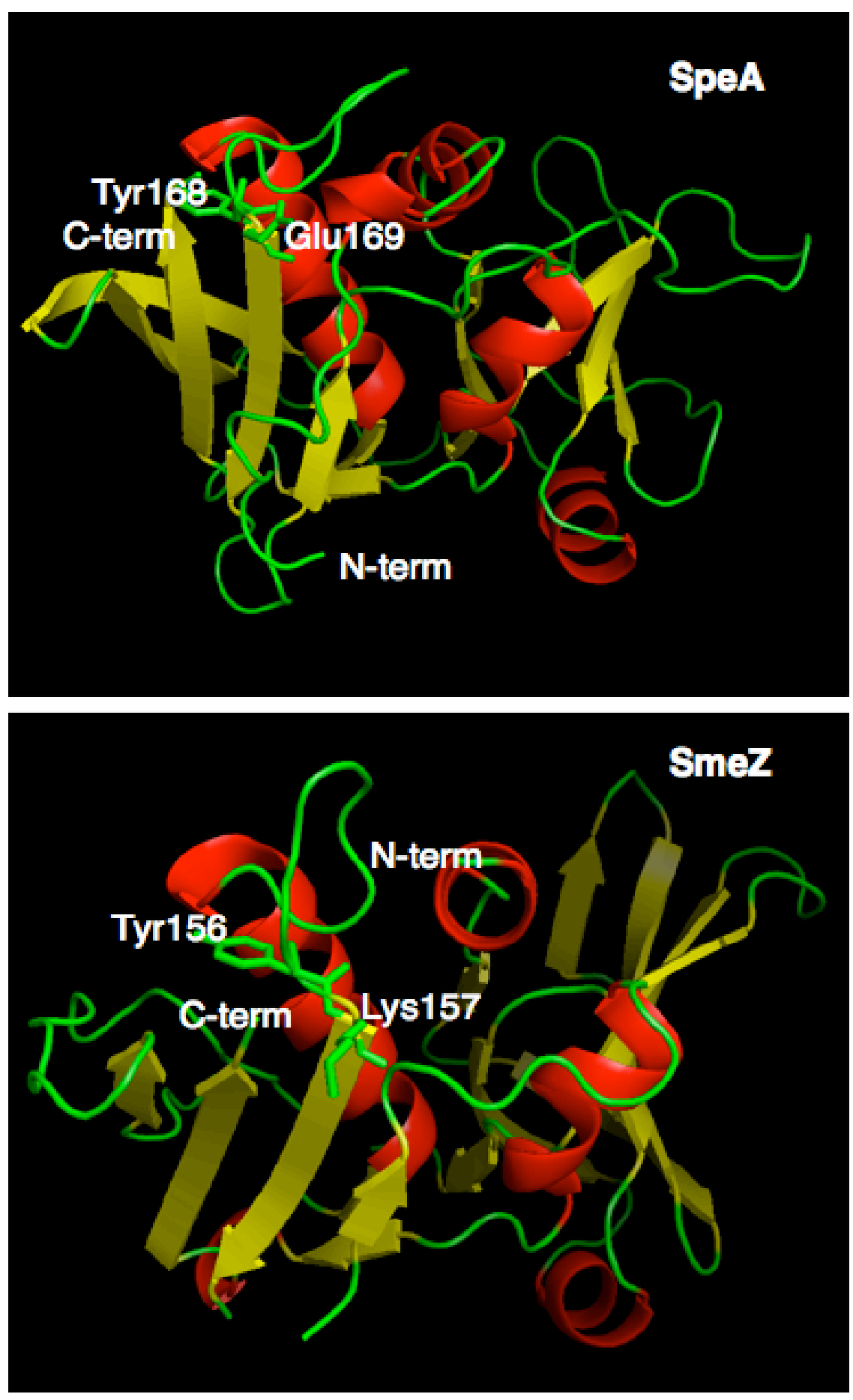

Figure 5.5 Ribbon diagram of the 3D structures of SpeA (upper) and SmeZ (lower).

Each diagram shows a flexible loop that matches the region predicted by disorder analysis (Fig 4); however, only the SmeZ loops has a lysine moiety likely to be at the SpeB cleavage site. 
its own processing to the enzymatically active $28-\mathrm{kDa}$ form, also known as 'streptopain' due to its close resemblance to papain. The enzyme is a cysteine protease belonging to a family of endopeptidases and shows a broad substrate specificity, including oxidized insulin B chain and other synthetic substrates (373). Although it is one of the earliest identified and most extensively studied streptococcal proteins, little is known about its cleavage sites. Most of the current knowledge about these cleavage sites comes from the enzyme's effects on the natural substrates i.e pro-SpeB, immunoglobulins, C5a peptidase, streptococcal proteins $M$ and $H(360,361,368)$ or synthetic peptides $(369)$, but no comprehensive studies have been performed to determine a consensus SpeB cleavage site. From the few studies performed, the preferred target for SpeB favors a hydrophobic residue (isoleucine or valine) at the $\mathrm{P}_{2}$ site and a positively charged residue (preferably lysine) at $\mathrm{P}_{1}(368,369)$. The only cleavage site that we could determine by $\mathrm{N}$-terminal sequencing in our studies happens to have isoleucine (I) and glutamic acid (E) at $\mathrm{P}_{2}$ and $\mathrm{P}_{1}$, respectively. However, bioinformatics analysis of SmeZ1 (and other SemZ homologs) revealed a conserved intrinsically disordered region containing a motif vulnerable to SpeB YKSG. This motif was different in SpeA2 (YETG) and was preceded by a proline moiety that makes susceptibility to protease unlikely. In both SpeJ and SpeG, the homologous motif was totally in an ordered region and expected to be folded. This notion that folded domains are protected from proteolysis is experimentally supported by the fact that tethering a histidine tag to the recombinant SAgs and incubating the taggedrSAgs with enzymatically active SpeB leads to a prompt degradation of the 'foreign' peptide linkers, generating products with identical N-termini, suggesting that these peptide linkers were exposed to the cysteine protease because they did not fit in the SAgs 
folding scheme. Accessibility of the proteolytic cleavage sites is also known to play an important role in the autoprocessing of pro-speB because four of the five auto-processing cleavage sites identified are located on accessible loops in the SpeB structure (374).

How does the selective degradation of SAgs affect the disease progression remains to be explored? Before moving from the in vitro observations to the in vivo implications, we have to address the question of what physiological concentrations these toxins achieve during infection. Since the concentrations of these SAgs achieved during infection are practically difficult to determine and are variable depending on the bacterial isolate and the site of bacterial infection, we decided to mix equal amounts of recombinant SAgs. At $200 \mu \mathrm{g} / \mathrm{ml}$, a concentration much higher than its physiological concentration (124), SmeZ1 could still be degraded by SpeB, and a SpeB concentration as low as $20 \mu \mathrm{g} / \mathrm{ml}$ was sufficient to breakdown SmeZ1 to below detectable limits. However, it is not uncommon that an enzyme may fail to act properly when the substrate level becomes sparse. Thus, to address the functional implications of the differential effects of SpeB on M1T1 SAgs, we compared its effects on the functionality of the susceptible rSmeZ1 vs the resistant rSpeA2. Using in vitro biochemical and biological assays, we showed that the selective degradation of SmeZ1 results in a significant loss of its superantigenic activity and its ability stimulate specific T-cells (Fig. 5.3A \& B), while all SpeA2 biological functions were practically unaffected.

The question remains: what happens during actual infections? A very relevant and complex question whose answer is controlled by at least three major factors, the coexpression of these proteins, their regulation and their 'available space.' In earlier studies, we showed that the relative expression of SpeA2 and SpeB protein and RNA is 
drastically and temporally reversed after animal passage (28). The differential expression of these proteins implies the presence of tight regulatory networks that affects their relative levels. High levels of SpeB expression in tissue and skin infection sites are in contrast with its complete shutdown in blood (73). In the initial phase of infection via skin or tissue, the bacteria need SpeB to break through the host tissue. Once the bacteria are in blood, SpeB expression can be detrimental to the bacteria as this protease degrades most of the bacterial anti-phagocytic proteins as well as the SAgs. $(69,375-377)$.

Selective degradation of GAS proteins by SpeB may explain why it is advantageous for the bacteria to down-regulate SpeB after the initial stages of infection, but it also explains what has been thought as "functional redundancy" of SAgic toxins in GAS. From an evolutionary aspect, it appears that GAS continues to acquire via horizontal gene transfer, more SAgs that seem to resist SpeB activity. The present study suggests that SpeA2 and SpeJ might be two such horizontally acquired SAgs because of 2 reasons: 1) their location on phage or transposable elements and 2) their absence in many sequenced GAS strains (http://www.nmpdr.org and Aziz etal; personal communication). Both SpeA and SpeJ are not shared by all strains [SpeJ is present in M1 (122) and M28 (378), and SpeA in only $20 \%$ of GAS strains (379)]. These superantigens also turn out to be most resistant to SpeB degradation and are able to keep their biological functions regardless whether SpeB is expressed or not. It is believed that non-specific immune responses elicited by the SAgs may allow a subset of the bacteria to escape specific immune defenses and make their way to host niches where they can cause chronic diseases such as rheumatic heart disease, glomerulonepharitis, psoriasis and pediatric neurological disorders (380). 


\title{
Chapter 6. Summary and Conclusions
}

\begin{abstract}
Summary
Overall, our study has provided valuable insight into how HLA class II allelic variation influences Strep SAgs responses that translates into different forms of GAS invasive infections being severe or non-severe. Before this study began and during its progress, most investigations involving HLA class II interaction with SAgs, though providing valuable information, revolved around demonstrating that distinct class II alleles differentially affect SAgs response in terms of proliferation or cytokine production, with only few studies addressing the molecular mechanisms for this phenomenon by investigating potential variability in HLA-SAg binding affinity (275, $284,285,287,289)$. Also, the previous studies mainly involved the early discovered staphylococcal SAgs (e.g. TSST-1, SEB, SEC), with only two or three Strep SAgs (primarily SpeB, SpeA, and SpeC) being investigated and few class II alleles that are not relevant to GAS pathogenesis $(288,290,297,381)$. On the other hand, our study has focused on translational research applications. We observed variation in different individuals' susceptibility to Strep SSD, SAg-mediated disease, and examined HLA class II-SAg interaction in vivo and in vitro, focusing on SAgs produced by the highly prevalent clonal M1T1 strain and on the class II haplotypes/alleles associated with different infection outcomes. Furthermore, we investigated the differential presentation of four Strep SAgs, individually or in mixture, by eleven prevalent haplotypes and eight single alleles, which makes our study more comprehensive and significant. This extensive screening aimed at revealing novel HLA-SAg interactions that could not be
\end{abstract}


detected in epidemiological association studies and at answering the questions: does the observed differential presentation apply to a particular SAg, a SAg combination, or to all SAgs, and does individuals' TCR V $\beta$ repertoires variation play a role in controlling SAg response and infection outcome or is HLA class II polymorphism the main player? Additionally, we sought to explore the molecular mechanisms for SAg-differential presentation by different class II alleles.

Using HLA tg mice, we validated the findings of the epidemiological association studies and showed the dominant effect of HLA class II on SAg responses in vivo and in vitro, an effect that was corroborated by our screening studies. Our screening demonstrated that all tested SAgs, individually or mixed, were preferentially presented by the high-risk allele/haplotype; however, some particularly interesting results were observed that will be detailed later.

Next, we examined the binding affinity of SAgs to different class II alleles with the aim of providing a molecular basis for their differential presentation. Although our binding results revealed a general agreement between SAgs binding to and presentation by different class II alleles, an exception was observed in case of SmeZ1 and DQ alleles. We went further in our molecular mechanistic studies by investigating biochemical events generated in $\mathrm{T}$ cells $\left(\mathrm{Ca}^{2+}\right.$ mobilization) when stimulated with SmeZ1 in the context of different DR or DQ alleles. Interestingly, the $\mathrm{Ca}^{2+}$ flux results supported the preferential presentation observations. These results may suggest that HLA-SAg affinity is only part of the process that modulate SAg responses, and that the overall avidity of the trimolecular complex (HLA-SAg-TCR) may determine the overall potency of the response. Another interesting finding of our study is that the protective alleles/haplotypes 
were found to be superior to the high-risk counterparts in inducing the regulatory

cytokine IL-10, which suggests that protection against Strep SSD may be mediated by an active rather than a passive effect.

\section{HLA-tg Mice Are Useful Animal Model for Studying SAg-mediated Diseases}

Epidemiological studies performed on patients with clear clinical manifestations are effective in revealing host immunogenetic factors that influence infection outcome; however, the drawn associations are questionable until validated in an appropriate animal model. We used HLA-tg mice expressing HLA-DQ6 (SSD protective allele) or HLADR4/DQ8 (SSD neutral alleles) to examine in vitro and in vivo responses to GAS SAgs. By using these mice, we achieved several goals. First, we overcame the poor response of traditional inbred mice to $\operatorname{SAgs}(296,297)$. Second, the high susceptibility of $\operatorname{tg}$ mice to SAg-mediated STSS allowed us to investigate the pathophysiology of STSS $(226,227$, 300, 301). Also, expression of distinct class II alleles in specific HLA-tg mice was useful for delineating the effect of class II allelic variation on Strep invasive infection outcome. Our in vitro findings showed that DQ6 splenocytes mounted remarkably lower responses to M1T1 SAgs than DR4/DQ8 splenocytes. Reproducibly, DQ6 mice elicited significantly lower inflammatory cytokine levels (TNF- $\alpha$ and IFN- $\gamma$ ) in vitro and in vivo than those of the DR4/DQ8 mice, and DQ6 mice were more resistant to infection than DR4/DQ8 mice. Altogether, these data provide a validation for our epidemiological association findings and confirm the protective effect of HLA-DQ6 allele.

Interestingly, both tg mouse lines had comparable bacterial load in liver and spleen. This observation underscores the role of HLA class II as a controller of the SAg- 
induced inflammatory cytokine responses and hence the severity of invasive GAS infection. However, this argument needs to be further addressed at late time points of infection when the death rate of DR4/DQ8 mice is higher. If the DQ6 and DR4/DQ8 mice demonstrate comparable bacteremia and tissue bacterial loads at these late stages of the disease, this will support our point that HLA class II polymorphism modulates the outcome of invasive GAS infection by governing the systemic inflammatory cytokine responses to SAgs rather than the bacterial clearance.

Also, another limitation of this study was the lack of HLA-tg mice expressing the high-risk alleles DR14, DQ5 or another protective allele DR15. Some of these mice are currently available (tg mice expressing DR14, and DR15) and the rest are being prepared, and we are planning to use them together with the tg mice described above to compare their responses to GAS SAgs in vivo and in vitro and to get a clearer picture of HLA allelic variation on Strep SAgs responses.

\section{Relative Contribution of HLA Class II Allelism and TCR V $\beta$ Repertoire Variation to Strep SAgs Responses}

SAg activity depends on interaction with both HLA class II molecules and TCR $\mathrm{V} \beta$ elements on APC and T cells, respectively. Therefore, in addition to the already demonstrated effect of HLA, SAg responses can also be influenced by the interaction with TCR V $\beta$ elements. Each SAg has a unique profile of TCR V $\beta$ specificity and interacts only with $\mathrm{T}$ cells expressing these $\mathrm{V} \beta$ elements. Therefore, SAg response can be affected by the relative abundance of SAg-specific TCR V $\beta$ families and the frequency of T cells expressing those families in a given individual's repertoire. These variables would 
influence the number of responding $\mathrm{T}$ cells in a given individual. Our SAg-cross presentation assays clearly demonstrated that TVR V $\beta$ repertoire variation has no effect on SAg response.

In HLA-tg mice, SAgs-cross presentation by either mouse line APC to each other $\mathrm{T}$ cells or to human $\mathrm{T}$ cells reproducibly demonstrated significant lower responses in the presence of the protective DQ6 APC as compared to DR4/DQ8 APC. Similarly, in humans with protective or high-risk alleles, SAg-cross presentation showed remarkably lower responses in the context of APC from the low responder irrespective of the T cell source. Furthermore, using B cell lines, homozygous for the protective alleles DR15/DQ6 or for the high-risk alleles DR14/DQ5, to present of the same Strep SAgs to T cells from different individuals, reproducibly resulted in significantly lower responses in the presence of the cell line with the protective alleles.

\section{HLA Class II Allelic Variation Determines Not Only the Magnitude But Also the Type of SAg-induced Cytokine Responses}

SAgs interact with both APC and T cells causing massive activation of large numbers of both cell types, which results in sudden cytokine storm. Such SAg-mediated cytokine responses induce the development of STSS. It was shown that individuals with the tendency to produce high levels of inflammatory cytokines develop severe systemic forms of GAS sepsis $(275,276)$. Our findings demonstrate that the magnitude of proinflammatory cytokines produced by splenocytes or by PBMC (stimulated with the same SAgs) expressing the protective alleles was remarkably lower than those expressing neutral or high-risk alleles. Similarly, in the SAg-cross presentation studies, we found 
that $\mathrm{T}$ cells from human or mice mount significantly lower level of pro-inflammatory cytokines if the SAgs were presented by APC expressing the protective alleles. Reproducibly, B cell lines homozygous for the protective haplotype (DR15/DQ6) or expressing single alleles from that haplotype induced lower level of pro-inflammatory cytokines when compared to B cell lines expressing high-risk haplotype/alleles (DR14/DQ5). Therefore, These data clearly indicate that of HLA class II allelic variation governs the magnitude of SAgs-triggered cytokine responses and thus determines the severity of GAS sepsis.

Interestingly, however, SAg-presentation by protective haplotype/alleles elicited high levels of the anti-inflammatory cytokine IL-10 that may have contributed to curbing the inflammatory responses and lowering $\mathrm{T}$ cell proliferation. This suggests that the protective effect of the DR15/DQ6 haplotype is not a passive action due to just poor SAg presentation. Rather, protection appears to be through active IL-10-mediated suppression of the inflammatory responses elicited by Strep SAgs. This view was supported by the fact that adding exogenous IL-10, which brought down the high-risk haplotype, induced SAgs-response to the same level of the protective haplotype. Although other studies have shown that SAgs can induce both pro- and anti-inflammatory cytokine responses (140, 382), we are the first to report differential production of these two types of cytokines in response to SAg-presentation by distinct class II haplotypes. These findings provide a molecular basis for the protective effect of DR15/DQ6 alleles in STSS, which appears to be mediated by increased IL-10 production and active suppression of inflammatory responses. 


\section{Developing a Sensitive System with Minimum Variables for Elucidating the Molecular Basis of Differential SAg-HLA Class II Interaction}

We tested the presentation of individual M1T1 rSAgs (SpeA-2, SpeG, SpeJ, and SmeZ1-1) by a panel of LBCL expressing HLA class II haplotypes that are prevalent in areas with high STSS incidence to find out which SAg is most affected with class II allelic variation. Our data showed that distinct class II haplotypes differentially presented all M1T1 rSAgs with the highest and lowest responses being triggered by the high-risk (DR14/DQ5) and the protective (DR15/DQ6) haplotype, respectively. The used BLCL normally express class II DR, DQ and DP isotypes. However, to delineate the contribution of individual alleles to SAg response single allele expressing cells are required. Furthermore, it is essential to eliminate any possible variability in the APC that may be related to any peculiar characters of the BLCL. Therefore, we generated a panel of BLS cell lines expressing single HLA DR or DQ alleles that are part of the high-risk, protective or neutral (neither high-risk nor protective) haplotypes. Such a generated panel would have different HLA DR or DQ alleles as the only variable contributed by the APC. To this end, we have established a simplified system that reduces the number of variables and maximizes our ability to dissect the effect of a single variable (different HLA class II alleles) at a time in modulating the molecular events and potency of SAgs, where the same SAg is presented to the same T cells by the same BLS expressing different class II alleles.

We used this system to determine whether the HLA class II effect on the SAg response is mediated by just one or if it requires both isotypes (DR and DQ) on the disease associated haplotypes. Our results showed that single class II alleles from 
protective or high-risk haplotypes presented individual M1T1 rSAgs in a way that mirrored the presentation of the same rSAgs by protective or high-risk haplotypes, respectively. The protective DR15 or DQ6 alleles elicited proliferative and cytokine response that significantly lower than those elicited by the high-risk DR14 or DQ5 alleles, while DR4, DR7, DQ2 and DQ7 elicited responses that are between these two extremes. Interestingly, DR14 and DQ5 were associated with the highest responses to all rSAgs except for SpeG, which demonstrated higher response in the presence of DQ7 than DQ5. This finding suggests that each SAg has its own way of interaction with class II alleles and that we cannot make a generalized statement about SAgs since exceptions always exist. Thus, we do not agree with the statement "if you have studied one SAg, you have studied them all". This view will be further substantiated by the results of the binding studies.

\section{Possible Molecular Mechanisms Underlying the Differential Effect of HLA Class II Molecules on SAgs Responses}

The stability of HLA-SAg interaction appears to be the earliest step that may modulate the downstream responses. Our binding studies results demonstrated that different binding affinity correlates with the observed functional responses. That is to say the protective class II alleles bind rSAg with lower affinity than that of the high-risk alleles. Therefore, the high-risk alleles could form greater numbers of SAg-MHC complexes or more stable complexes on the APC and consequently cause more pronounced T proliferation and cytokine production. This was found to be true for SpeG and SpeJ binding to different HLA DR or DQ alleles. However, SmeZ1 lacked the 
correlation of binding to and presentation by distinct HLA DQ alleles. SmeZ1 binding to the high risk HLA DQ5 allele was weaker than that to the protective HLA DQ6. The disagreement between proliferation and binding data for SmeZ1 in the context of HLA DQ alleles, may suggest that the overall avidity of the trimolecular complex (HLA-SAgTCR) is the main governing factor. We addressed this possibility by studying $\mathrm{Ca}^{2+}$ mobilization in T cells following stimulation with SmeZ1 in the presence of different HLA DR or DQ alleles. Interestingly, presentation of SmeZ1 by the high-risk HLA DQ5 allele induced remarkably higher $\mathrm{Ca}^{2+}$ influx than that induced by the protective HLA DQ6 alleles. Such finding suggests that the responses to SmeZ1 in the context of HLA DQ alleles may be controlled by the high affinity of TCR V $\beta$ elements for DQ-SmeZ1 complex rather than the mere stability of that complex. In other words, the stability of the HLA DQ5-SmeZ1-TCR ternary complex depends on the stability of both SmeZ1-TCR and DQ5-SmeZ1 interactions, with the former being relatively more important in this particular case.

Collectively, the findings presented in this dissertation emphasize the dominant and direct role of HLA class II as a regulator of Strep SAgs responses, which modulates disease severity. Furthermore, it is clear that each SAg-HLA class II allele interaction is distinctive. However, as mentioned earlier, along with class II polymorphism other factors may also affect SAg responses; including how a SAg interacts with HLA class II molecule (via high affinity $\beta$-chain or low affinity $\alpha$-chain) and whether it can crosslink class II molecules. Also, the nature of class II bound peptide can affect SAg presentation. Different biological compartments vary in resident APC with distinct repertoires of class II-bound peptides; some of these peptides permit SAg presentation while others do not. 
TCR also plays an important role through its effect on the stability of the trimolecular complex of HLA-SAg-TCR; an effect that varies with the SAg, TCR V $\beta$ element and in particular the class II allele involved. All these factors are not mutually exclusive and may act together to potentiate SAgs responses in different individuals and tissues.

Another layer of complexity is added if we consider that other host polymorphic genes may play a role in regulating SAg response as well as other bacterial components that interact with SAg and are likely to contribute to the pathogenesis of GAS sepsis as well.

\section{Bacterial Factors Affecting Strep SAgs Responses}

So far, we have focused on host factors that control Strep SAgs responses. However, in vivo the bacteria produce many other virulence factors that interact with each other and with the host immune system. Therefore, in the real life situation, in addition to Strep SAgs, the host experiences several other GAS virulence factors including the major streptococcal cysteine protease SpeB whose expression results in partial loss of the immune-stimulating activity of the native secreted Strep SAgs produced by the globally disseminated M1T1 strain (363). Therefore, we examined the susceptibility of each of the M1T1 rSAgs to degradation by rSpeB. Our data have shown that SmeZ1 was degraded completely whereas SpeG and SpeA2 were more resistant and SpeJ was completely unaffected by the proteolytic effects of SpeB. As expected, the degradation of SmeZ1 abolished its immune-stimulatory activity in vitro and in vivo. We already know that, in invasive cases, the clonal M1T1 strain turns off SpeB to spare M protein and other virulence factors important for survival and evasion of host immune 
system $(29,383)$. These data point to smeZ1 as a highly crucial SAg that the bacteria have to spare.

\section{Future Directions}

\section{Examine IL-10 as the primary mediator of protective alleles suppressive effects}

Our studies in chapter 3 and 4 suggested that IL-10 may mediate the suppressive effects of the protective HLA class II alleles, where addition of exogenous rIL-10 lowered high-risk alleles-induced SAgs response to a level comparable to that of protective alleles. To further explore this hypothesis, we will:

1. Add different doses of rIL-10 and observe whether this would result in a dosedependent reduction of SAgs response when presented by high-risk alleles.

2. Add neutralizing anti-IL-10 Ab to culture containing APC expressing the protective alleles and examine whether this would increase SAg-mediated responses to that induced by high-risk alleles.

3. Add anti-proinflammatory cytokines Abs (e.g. Anti-INF- $\gamma,-$ TNF- $\alpha$ and $-\beta$ ) to cultures containing APC expressing high-risk alleles and examine whether this would decrease SAg-triggered responses to a level comparable to that of the protective alleles.

4. In vitro, assess IL-10 produced by SAgs-stimulated splenocytes from HLA-tg mice expressing protective (DQ6) or neutral (DR4/DQ8) alleles. If we find significant difference in IL-10 production by DQ6 than by DR4/DQ8 splenocytes, then we will perform a similar set of experiments to that stated in 2 and 3. 
5. Assess IL-10 level in the plasma of HLA-tg mice expressing protective (DQ6) or neutral (DR4/DQ8) alleles following i.v., infection with life M1T1 GAS. If DQ6 mice elicit higher levels of IL-10 than DR4/DQ8 mice, then we will examine whether administration of rIL-10 to DR4/DQ8 mice or anti-IL-10 to DQ6 mice would improve the survival of the former or increase the death rate of the later.

If our in vitro and in vivo results demonstrate that addition or administration rIL10 in the context of high-risk alleles reduces SAg-triggered inflammatory responses to a level comparable to that observed in the context og high risk alleles, this would suggest that IL-10 may be an important contributor to the protection mediated by DR15 and DQ6 alleles. On the other hand, if the in vitro and in vivo use of neutralizing anti-IL-10 Ab in the presence of protective alleles enhances SAgs responses in a way equivalent to that demonstrated in he presence of high-risk alleles, this would confirm our hypothesis that the protective effects of DR15/DQ6 alleles are mediated by an active IL-10-mediated suppression of the SAg-induced inflammatory responses.

\section{Study the interaction of protective and high-risk alleles in modulating SAgs}

\section{responses}

We will assess the impact of HLA class II hetero- vs. homozygosity with respect to the risk/protection status of an individual. We will generate BLS cells expressing two alleles from different haplotypes and determine if the effects of the protective alleles dominate over those of the high risk alleles or vice versa. We will use BLS cells expressing DRB1*1501 or DQB1*06 in conjunction with DRB1*1401 or DQB1*0503 expressed on different cells or co-expressed on the same cells. This information is 
relevant to real life infections in heterozygous individuals, where the effect of a particular allele or haplotype can be modulated in the presence of another

If in the presence of both DQ6 and DR14 we find $>50 \%$ reduction in response compared to cultures with DR14 \pm DQ5, this would suggest active suppression of the SAg response by the protective DQ6 allele. Alternatively, the effects of risk alleles are dominant, or that the risk and protective alleles will neutralize each other's effect to the point where the response becomes comparable to that with neutral alleles. The results obtained from these experiments will help us determine whether individuals with protective alleles co-expressed with high-risk alleles are as protected against STSS. These distinctions are important for translating our findings to predict host susceptibility to severe GAS sepsis.

\section{Validate our in vitro findings using HLA-transgenic mice expressing individual or combinations of HLA class II alleles}

The goal here is to create an animal model that can be used to validate in vitro findings, and allow us to conduct in-depth mechanistic studies in vivo. For this study we need a set of HLA-tg mice expressing DRB1*1501, DQA1*0102-DQB1*0602, DRB1*1401, DQA1*0104-DQB1*0503, DRB1*0701, DQA1*0201-DQB1*0201 and DRB1*0402-DQA $1 * 0301-D Q B 1 * 0302$ because these alleles are present in haplotypes associated with outcomes of GAS sepsis in humans as demonstrated by epidemiological study (275). Some of these mice are currently available (DR15, DR3, DR4, DR7) and we are breeding and characterizing them as well as working on generating the rest of the required mice. Using these mice, we will validate the in vitro data generated with the 
BLS lines and analyze the interactive effects of protective and high-risk alleles on the systemic response to SAgs and on the severity of GAS sepsis in HLA-tg mice. We predict that the systemic inflammatory response elicited by the same dose of SAgs or whole bacteria will vary considerably depending on HLA class II alleles expressed by the mice. We expect that systemic inflammatory responses in mice expressing the high-riskassociated class II alleles will be significantly higher than in mice expressing protective alleles. Further, we expect that mice expressing both protective and high-risk alleles will be protected from the severe systemic manifestation triggered by SAgs or whole bacteria. Our predictions are supported by the data generated with the HLA-tg mice used in the current study. If as we expect, we see a confirmation of these results in the newly generated mice, this will support our hypothesis of the dominance of the protective class II alleles 


\section{LIST OF REFERENCES}

1. Boyle, M. D., and R. Lottenberg. 1997. Plasminogen activation by invasive human pathogens. Thromb Haemost 77:1-10.

2. Lijnen, H. R., F. Bachmann, D. Collen, V. Ellis, H. Pannekoek, D. C. Rijken, and S. Thorsen. 1994. Mechanisms of plasminogen activation. J Intern Med 236:415424.

3. Brown, J. H. 1919. The use of blood agar for the study of streptococci. In In Rockefeller Institute for Medical Research Monograph No 9., Vol. No 9.

4. Lancefield, R. C. 1933. A serological differentiation of human and other groups of hemolytic streptococci. J Exp Med 57:571-595.

5. Lancefield, R. C. 1962. Current knowledge of type-specific M antigens of group A streptococci. J Immunol 89:307-313.

6. Aziz, R. K., R. A. Edwards, W. W. Taylor, D. E. Low, A. McGeer, and M. Kotb. 2005. Mosaic prophages with horizontally acquired genes account for the emergence and diversification of the globally disseminated M1T1 clone of Streptococcus pyogenes. J Bacteriol 187:3311-3318.

7. Kotb, M., A. Norrby-Teglund, A. McGeer, K. Green, and D. E. Low. 2003. Association of human leukocyte antigen with outcomes of infectious diseases: the streptococcal experience. Scand J Infect Dis 35:665-669.

8. McMillan, D. J., and K. S. Sriprakash. 2007. Virulence Factors and Their Regulation in Group A Streptococcus. Horizon Bioscience.

9. Cunningham, M. W. n. 2000. Pathogenesis of group A streptococcal infections. Clin Microbiol Rev 13:470-511.

10. Cunningham, M. W. 2003. Autoimmunity and molecular mimicry in the pathogenesis of post-streptococcal heart disease. Front Biosci 8:s533-543.

11. Stollerman, G. H. 2002. Current issues in the prevention of rheumatic fever. Minerva Med 93:371-387.

12. Asbahr, F. R., M. A. Garvey, L. A. Snider, D. M. Zanetta, H. Elkis, and S. E. Swedo. 2005. Obsessive-compulsive symptoms among patients with Sydenham chorea. Biol Psychiatry 57:1073-1076.

13. Ben-Pazi, H., A. Livne, Y. Shapira, and R. C. Dale. 2003. Parkinsonian features after streptococcal pharyngitis. J Pediatr 143:267-269.

14. Kurlan, R., and E. L. Kaplan. 2004. The pediatric autoimmune neuropsychiatric disorders associated with streptococcal infection (PANDAS) etiology for tics and obsessive-compulsive symptoms: hypothesis or entity? Practical considerations for the clinician. Pediatrics 113:883-886.

15. Snider, L. A., and S. E. Swedo. 2003. Post-streptococcal autoimmune disorders of the central nervous system. Curr Opin Neurol 16:359-365.

16. Carapetis, J. R., A. C. Steer, E. K. Mulholland, and M. Weber. 2005. The global burden of group A streptococcal diseases. Lancet Infect Dis 5:685-694.

17. Tart, A. H., M. J. Walker, and J. M. Musser. 2007. New understanding of the group A Streptococcus pathogenesis cycle. Trends Microbiol 15:318-325. 
18. Hoge, C. W., B. Schwartz, D. F. Talkington, R. F. Breiman, E. M. MacNeill, and S. J. Englender. 1993. The changing epidemiology of invasive group A streptococcal infections and the emergence of streptococcal toxic shock-like syndrome. A retrospective population-based study. Jama 269:384-389.

19. Low, D. E., B. Schwartz, and A. McGeer. 1997. The reemergence of severe group A streptococcal disease. In emerging infections, Vol. 1. w. Scheld, D. Armstrong, and j. hughes, eds. ASM press, Washington, DC, p. 93-123.

20. Sharkawy, A., D. E. Low, R. Saginur, D. Gregson, B. Schwartz, P. Jessamine, K. Green, and A. McGeer. 2002. Severe group a streptococcal soft-tissue infections in Ontario: 1992-1996. Clin Infect Dis 34:454-460.

21. Vlaminckx, B., W. van Pelt, L. Schouls, A. van Silfhout, C. Elzenaar, E. Mascini, J. Verhoef, and J. Schellekens. 2004. Epidemiological features of invasive and noninvasive group A streptococcal disease in the Netherlands, 1992-1996. Eur J Clin Microbiol Infect Dis 23:434-444.

22. Davies, H. D., A. McGeer, B. Schwartz, K. Green, D. Cann, A. E. Simor, and D. E. Low. 1996. Invasive group A streptococcal infections in Ontario, Canada. Ontario Group A Streptococcal Study Group. N Engl J Med 335:547-554.

23. Eriksson, B. K., J. Andersson, S. E. Holm, and M. Norgren. 1999. Invasive group A streptococcal infections: T1M1 isolates expressing pyrogenic exotoxins A and $\mathrm{B}$ in combination with selective lack of toxin-neutralizing antibodies are associated with increased risk of streptococcal toxic shock syndrome. J Infect Dis 180:410-418.

24. Cleary, P. P., D. LaPenta, R. Vessela, H. Lam, and D. Cue. 1998. A globally disseminated M1 subclone of group A streptococci differs from other subclones by 70 kilobases of prophage DNA and capacity for high-frequency intracellular invasion. Infect Immun 66:5592-5597.

25. Cockerill, F. R., 3rd, K. L. MacDonald, R. L. Thompson, F. Roberson, P. C. Kohner, J. Besser-Wiek, J. M. Manahan, J. M. Musser, P. M. Schlievert, J. Talbot, B. Frankfort, J. M. Steckelberg, W. R. Wilson, and M. T. Osterholm. 1997. An outbreak of invasive group A streptococcal disease associated with high carriage rates of the invasive clone among school-aged children. Jama 277:38-43.

26. Aziz, R. K., S. A. Ismail, H. W. Park, and M. Kotb. 2004. Post-proteomic identification of a novel phage-encoded streptodornase, Sda1, in invasive M1T1 Streptococcus pyogenes. Mol Microbiol 54:184-197.

27. Sumby, P., S. F. Porcella, A. G. Madrigal, K. D. Barbian, K. Virtaneva, S. M. Ricklefs, D. E. Sturdevant, M. R. Graham, J. Vuopio-Varkila, N. P. Hoe, and J. M. Musser. 2005. Evolutionary origin and emergence of a highly successful clone of serotype M1 group a Streptococcus involved multiple horizontal gene transfer events. J Infect Dis 192:771-782.

28. Kazmi, S. U., R. Kansal, R. K. Aziz, M. Hooshdaran, A. Norrby-Teglund, D. E. Low, A. B. Halim, and M. Kotb. 2001. Reciprocal, temporal expression of SpeA and SpeB by invasive M1T1 group a streptococcal isolates in vivo. Infect Immun 69:4988-4995.

29. Walker, M. J., A. Hollands, M. L. Sanderson-Smith, J. N. Cole, J. K. Kirk, A. Henningham, J. D. McArthur, K. Dinkla, R. K. Aziz, R. G. Kansal, A. J. Simpson, J. T. Buchanan, G. S. Chhatwal, M. Kotb, and V. Nizet. 2007. DNase 
Sda1 provides selection pressure for a switch to invasive group A streptococcal infection. Nat Med 13:981-985.

30. Herwald, H., H. Cramer, M. Morgelin, W. Russell, U. Sollenberg, A. NorrbyTeglund, H. Flodgaard, L. Lindbom, and L. Bjorck. 2004. M protein, a classical bacterial virulence determinant, forms complexes with fibrinogen that induce vascular leakage. Cell 116:367-379.

31. Pahlman, L. I., M. Morgelin, J. Eckert, L. Johansson, W. Russell, K. Riesbeck, O. Soehnlein, L. Lindbom, A. Norrby-Teglund, R. R. Schumann, L. Bjorck, and H. Herwald. 2006. Streptococcal M protein: a multipotent and powerful inducer of inflammation. J Immunol 177:1221-1228.

32. Shannon, O., E. Hertzen, A. Norrby-Teglund, M. Morgelin, U. Sjobring, and L. Bjorck. 2007. Severe streptococcal infection is associated with M protein-induced platelet activation and thrombus formation. Mol Microbiol 65:1147-1157.

33. Pahlman, L. I., A. I. Olin, J. Darenberg, M. Morgelin, M. Kotb, H. Herwald, and A. Norrby-Teglund. 2008. Soluble M1 protein of Streptococcus pyogenes triggers potent T cell activation. Cell Microbiol 10:404-414.

34. Tomai, M., M. Kotb, G. Majumdar, and E. H. Beachey. 1990. Superantigenicity of streptococcal M protein. J Exp Med 172:359-362.

35. Mitchell, T. J. 2003. The pathogenesis of streptococcal infections: from tooth decay to meningitis. Nat Rev Microbiol 1:219-230.

36. Hasty, D. L., I. Ofek, H. S. Courtney, and R. J. Doyle. 1992. Multiple adhesins of streptococci. Infect Immun 60:2147-2152.

37. Kristian, S. A., V. Datta, C. Weidenmaier, R. Kansal, I. Fedtke, A. Peschel, R. L. Gallo, and V. Nizet. 2005. D-alanylation of teichoic acids promotes group a streptococcus antimicrobial peptide resistance, neutrophil survival, and epithelial cell invasion. J Bacteriol 187:6719-6725.

38. Dale, J. B., R. G. Washburn, M. B. Marques, and M. R. Wessels. 1996. Hyaluronate capsule and surface $M$ protein in resistance to opsonization of group A streptococci. Infect Immun 64:1495-1501.

39. Dinkla, K., M. Rohde, W. T. Jansen, E. L. Kaplan, G. S. Chhatwal, and S. R. Talay. 2003. Rheumatic fever-associated Streptococcus pyogenes isolates aggregate collagen. J Clin Invest 111:1905-1912.

40. Schrager, H. M., S. Alberti, C. Cywes, G. J. Dougherty, and M. R. Wessels. 1998. Hyaluronic acid capsule modulates $M$ protein-mediated adherence and acts as a ligand for attachment of group A Streptococcus to CD44 on human keratinocytes. J Clin Invest 101:1708-1716.

41. Medina, E., G. Molinari, M. Rohde, B. Haase, G. S. Chhatwal, and C. A. Guzman. 1999. Fc-mediated nonspecific binding between fibronectin-binding protein I of Streptococcus pyogenes and human immunoglobulins. J Immunol 163:3396-3402.

42. Jadoun, J., V. Ozeri, E. Burstein, E. Skutelsky, E. Hanski, and S. Sela. 1998. Protein F1 is required for efficient entry of Streptococcus pyogenes into epithelial cells. J Infect Dis 178:147-158.

43. Dinkla, K., M. Rohde, W. T. Jansen, J. R. Carapetis, G. S. Chhatwal, and S. R. Talay. 2003. Streptococcus pyogenes recruits collagen via surface-bound 
fibronectin: a novel colonization and immune evasion mechanism. Mol Microbiol 47:861-869.

44. Cleary, P. P., U. Prahbu, J. B. Dale, D. E. Wexler, and J. Handley. 1992.

Streptococcal C5a peptidase is a highly specific endopeptidase. Infect Immun 60:5219-5223.

45. Edwards, R. J., G. W. Taylor, M. Ferguson, S. Murray, N. Rendell, A. Wrigley, Z. Bai, J. Boyle, S. J. Finney, A. Jones, H. H. Russell, C. Turner, J. Cohen, L. Faulkner, and S. Sriskandan. 2005. Specific C-terminal cleavage and inactivation of interleukin- 8 by invasive disease isolates of Streptococcus pyogenes. J Infect Dis 192:783-790.

46. Sumby, P., S. Zhang, A. R. Whitney, F. Falugi, G. Grandi, E. A. Graviss, F. R. Deleo, and J. M. Musser. 2008. A chemokine-degrading extracellular protease made by group A Streptococcus alters pathogenesis by enhancing evasion of the innate immune response. Infect Immun 76:978-985.

47. Jones, C. H., T. C. Bolken, K. F. Jones, G. O. Zeller, and D. E. Hruby. 2001. Conserved DegP protease in gram-positive bacteria is essential for thermal and oxidative tolerance and full virulence in Streptococcus pyogenes. Infect Immun 69:5538-5545.

48. Cole, J. N., J. A. Aquilina, P. G. Hains, A. Henningham, K. S. Sriprakash, M. G. Caparon, V. Nizet, M. Kotb, S. J. Cordwell, S. P. Djordjevic, and M. J. Walker. 2007. Role of group A Streptococcus HtrA in the maturation of SpeB protease. Proteomics 7:4488-4498.

49. Lyon, W. R., and M. G. Caparon. 2004. Role for serine protease HtrA (DegP) of Streptococcus pyogenes in the biogenesis of virulence factors SpeB and the hemolysin streptolysin S. Infect Immun 72:1618-1625.

50. Berggard, K., E. Johnsson, E. Morfeldt, J. Persson, M. Stalhammar-Carlemalm, and G. Lindahl. 2001. Binding of human C4BP to the hypervariable region of $\mathrm{M}$ protein: a molecular mechanism of phagocytosis resistance in Streptococcus pyogenes. Mol Microbiol 42:539-551.

51. Horstmann, R. D., H. J. Sievertsen, J. Knobloch, and V. A. Fischetti. 1988. Antiphagocytic activity of streptococcal M protein: selective binding of complement control protein factor H. Proc Natl Acad Sci U S A 85:1657-1661.

52. Horstmann, R. D., H. J. Sievertsen, M. Leippe, and V. A. Fischetti. 1992. Role of fibrinogen in complement inhibition by streptococcal M protein. Infect Immun 60:5036-5041.

53. Staali, L., S. Bauer, M. Morgelin, L. Bjorck, and H. Tapper. 2006. Streptococcus pyogenes bacteria modulate membrane traffic in human neutrophils and selectively inhibit azurophilic granule fusion with phagosomes. Cell Microbiol 8:690-703.

54. Courtney, H. S., C. von Hunolstein, J. B. Dale, M. S. Bronze, E. H. Beachey, and D. L. Hasty. 1992. Lipoteichoic acid and M protein: dual adhesins of group A streptococci. Microb Pathog 12:199-208.

55. Cue, D., P. E. Dombek, H. Lam, and P. P. Cleary. 1998. Streptococcus pyogenes serotype M1 encodes multiple pathways for entry into human epithelial cells. Infect Immun 66:4593-4601. 
56. Carlsson, F., K. Berggard, M. Stalhammar-Carlemalm, and G. Lindahl. 2003.

Evasion of phagocytosis through cooperation between two ligand-binding regions in Streptococcus pyogenes M protein. J Exp Med 198:1057-1068.

57. Podbielski, A., N. Schnitzler, P. Beyhs, and M. D. Boyle. 1996. M-related protein (Mrp) contributes to group A streptococcal resistance to phagocytosis by human granulocytes. Mol Microbiol 19:429-441.

58. Visai, L., S. Bozzini, G. Raucci, A. Toniolo, and P. Speziale. 1995. Isolation and characterization of a novel collagen-binding protein from Streptococcus pyogenes strain 6414. J Biol Chem 270:347-353.

59. Lei, B., L. M. Smoot, H. M. Menning, J. M. Voyich, S. V. Kala, F. R. Deleo, S. D. Reid, and J. M. Musser. 2002. Identification and characterization of a novel heme-associated cell surface protein made by Streptococcus pyogenes. Infect Immun 70:4494-4500.

60. Lukomski, S., K. Nakashima, I. Abdi, V. J. Cipriano, R. M. Ireland, S. D. Reid, G. G. Adams, and J. M. Musser. 2000. Identification and characterization of the scl gene encoding a group A Streptococcus extracellular protein virulence factor with similarity to human collagen. Infect Immun 68:6542-6553.

61. Han, R., C. C. Caswell, E. Lukomska, D. R. Keene, M. Pawlowski, J. M. Bujnicki, J. K. Kim, and S. Lukomski. 2006. Binding of the low-density lipoprotein by streptococcal collagen-like protein Scl1 of Streptococcus pyogenes. Mol Microbiol 61:351-367.

62. Caswell, C. C., E. Lukomska, N. S. Seo, M. Hook, and S. Lukomski. 2007. Scl1dependent internalization of group A Streptococcus via direct interactions with the alpha2beta(1) integrin enhances pathogen survival and re-emergence. Mol Microbiol 64:1319-1331.

63. Elsner, A., B. Kreikemeyer, A. Braun-Kiewnick, B. Spellerberg, B. A. Buttaro, and A. Podbielski. 2002. Involvement of Lsp, a member of the LraI-lipoprotein family in Streptococcus pyogenes, in eukaryotic cell adhesion and internalization. Infect Immun 70:4859-4869.

64. Hirota, K., H. Kanitani, K. Nemoto, T. Ono, and Y. Miyake. 1995. Crossreactivity between human sialyl Lewis(x) oligosaccharide and common causative oral bacteria of infective endocarditis. FEMS Immunol Med Microbiol 12:159164.

65. Oehmcke, S., A. Podbielski, and B. Kreikemeyer. 2004. Function of the fibronectin-binding serum opacity factor of Streptococcus pyogenes in adherence to epithelial cells. Infect Immun 72:4302-4308.

66. Reid, S. D., A. G. Montgomery, J. M. Voyich, F. R. DeLeo, B. Lei, R. M. Ireland, N. M. Green, M. Liu, S. Lukomski, and J. M. Musser. 2003. Characterization of an extracellular virulence factor made by group A Streptococcus with homology to the Listeria monocytogenes internalin family of proteins. Infect Immun 71:7043-7052.

67. Berge, A., B. M. Kihlberg, A. G. Sjoholm, and L. Bjorck. 1997. Streptococcal protein $\mathrm{H}$ forms soluble complement-activating complexes with IgG, but inhibits complement activation by IgG-coated targets. J Biol Chem 272:20774-20781. 
68. Brenot, A., K. Y. King, B. Janowiak, O. Griffith, and M. G. Caparon. 2004. Contribution of glutathione peroxidase to the virulence of Streptococcus pyogenes. Infect Immun 72:408-413.

69. Rasmussen, M., H. P. Muller, and L. Bjorck. 1999. Protein GRAB of streptococcus pyogenes regulates proteolysis at the bacterial surface by binding alpha2-macroglobulin. J Biol Chem 274:15336-15344.

70. Derbise, A., Y. P. Song, S. Parikh, V. A. Fischetti, and V. Pancholi. 2004. Role of the C-terminal lysine residues of streptococcal surface enolase in Glu- and Lysplasminogen-binding activities of group A streptococci. Infect Immun 72:94-105.

71. McKay, F. C., J. D. McArthur, M. L. Sanderson-Smith, S. Gardam, B. J. Currie, K. S. Sriprakash, P. K. Fagan, R. J. Towers, M. R. Batzloff, G. S. Chhatwal, M. Ranson, and M. J. Walker. 2004. Plasminogen binding by group A streptococcal isolates from a region of hyperendemicity for streptococcal skin infection and a high incidence of invasive infection. Infect Immun 72:364-370.

72. Terao, Y., M. Yamaguchi, S. Hamada, and S. Kawabata. 2006. Multifunctional glyceraldehyde-3-phosphate dehydrogenase of Streptococcus pyogenes is essential for evasion from neutrophils. J Biol Chem 281:14215-14223.

73. Cole, J. N., J. D. McArthur, F. C. McKay, M. L. Sanderson-Smith, A. J. Cork, M. Ranson, M. Rohde, A. Itzek, H. Sun, D. Ginsburg, M. Kotb, V. Nizet, G. S. Chhatwal, and M. J. Walker. 2006. Trigger for group A streptococcal M1T1 invasive disease. Faseb J 20:1745-1747.

74. Lottenberg, R., D. Minning-Wenz, and M. D. Boyle. 1994. Capturing host plasmin(ogen): a common mechanism for invasive pathogens? Trends Microbiol 2:20-24.

75. Collin, M., and A. Olsen. 2001. Effect of SpeB and EndoS from Streptococcus pyogenes on human immunoglobulins. Infect Immun 69:7187-7189.

76. Hynes, W. L., and S. L. Walton. 2000. Hyaluronidases of Gram-positive bacteria. FEMS Microbiol Lett 183:201-207.

77. Buchanan, J. T., A. J. Simpson, R. K. Aziz, G. Y. Liu, S. A. Kristian, M. Kotb, J. Feramisco, and V. Nizet. 2006. DNase expression allows the pathogen group A Streptococcus to escape killing in neutrophil extracellular traps. Curr Biol 16:396-400.

78. Collin, M., M. D. Svensson, A. G. Sjoholm, J. C. Jensenius, U. Sjobring, and A. Olsen. 2002. EndoS and SpeB from Streptococcus pyogenes inhibit immunoglobulin-mediated opsonophagocytosis. Infect Immun 70:6646-6651.

79. Nyberg, P., M. Rasmussen, and L. Bjorck. 2004. alpha2-Macroglobulinproteinase complexes protect Streptococcus pyogenes from killing by the antimicrobial peptide LL-37. J Biol Chem 279:52820-52823.

80. Burns, E. H., Jr., A. M. Marciel, and J. M. Musser. 1996. Activation of a 66kilodalton human endothelial cell matrix metalloprotease by Streptococcus pyogenes extracellular cysteine protease. Infect Immun 64:4744-4750.

81. Herwald, H., M. Collin, W. Muller-Esterl, and L. Bjorck. 1996. Streptococcal cysteine proteinase releases kinins: a virulence mechanism. J Exp Med 184:665673.

82. Kapur, V., M. W. Majesky, L. L. Li, R. A. Black, and J. M. Musser. 1993. Cleavage of interleukin 1 beta (IL-1 beta) precursor to produce active IL-1 beta 
by a conserved extracellular cysteine protease from Streptococcus pyogenes. Proc Natl Acad Sci U S A 90:7676-7680.

83. Kapur, V., S. Topouzis, M. W. Majesky, L. L. Li, M. R. Hamrick, R. J. Hamill, J. M. Patti, and J. M. Musser. 1993. A conserved Streptococcus pyogenes extracellular cysteine protease cleaves human fibronectin and degrades vitronectin. Microb Pathog 15:327-346.

84. Akesson, P., L. Moritz, M. Truedsson, B. Christensson, and U. von PawelRammingen. 2006. IdeS, a highly specific immunoglobulin G (IgG)-cleaving enzyme from Streptococcus pyogenes, is inhibited by specific IgG antibodies generated during infection. Infect Immun 74:497-503.

85. Lei, B., F. R. DeLeo, N. P. Hoe, M. R. Graham, S. M. Mackie, R. L. Cole, M. Liu, H. R. Hill, D. E. Low, M. J. Federle, J. R. Scott, and J. M. Musser. 2001. Evasion of human innate and acquired immunity by a bacterial homolog of CD11b that inhibits opsonophagocytosis. Nat Med 7:1298-1305.

86. Fernie-King, B. A., D. J. Seilly, C. Willers, R. Wurzner, A. Davies, and P. J. Lachmann. 2001. Streptococcal inhibitor of complement (SIC) inhibits the membrane attack complex by preventing uptake of C567 onto cell membranes. Immunology 103:390-398.

87. Fernie-King, B. A., D. J. Seilly, and P. J. Lachmann. 2004. The interaction of streptococcal inhibitor of complement (SIC) and its proteolytic fragments with the human beta defensins. Immunology 111:444-452.

88. Hoe, N. P., R. M. Ireland, F. R. DeLeo, B. B. Gowen, D. W. Dorward, J. M. Voyich, M. Liu, E. H. Burns, Jr., D. M. Culnan, A. Bretscher, and J. M. Musser. 2002. Insight into the molecular basis of pathogen abundance: group A Streptococcus inhibitor of complement inhibits bacterial adherence and internalization into human cells. Proc Natl Acad Sci U S A 99:7646-7651.

89. Datta, V., S. M. Myskowski, L. A. Kwinn, D. N. Chiem, N. Varki, R. G. Kansal, M. Kotb, and V. Nizet. 2005. Mutational analysis of the group A streptococcal operon encoding streptolysin $\mathrm{S}$ and its virulence role in invasive infection. $\mathrm{Mol}$ Microbiol 56:681-695.

90. Sierig, G., C. Cywes, M. R. Wessels, and C. D. Ashbaugh. 2003. Cytotoxic effects of streptolysin o and streptolysin s enhance the virulence of poorly encapsulated group a streptococci. Infect Immun 71:446-455.

91. Bricker, A. L., C. Cywes, C. D. Ashbaugh, and M. R. Wessels. 2002. NAD+glycohydrolase acts as an intracellular toxin to enhance the extracellular survival of group A streptococci. Mol Microbiol 44:257-269.

92. Baschieri, S., R. K. Lees, A. R. Lussow, and H. R. MacDonald. 1993. Clonal anergy to staphylococcal enterotoxin B in vivo: selective effects on $\mathrm{T}$ cell subsets and lymphokines. Eur J Immunol 23:2661-2666.

93. MacDonald, H. R., R. K. Lees, S. Baschieri, T. Herrmann, and A. R. Lussow. 1993. Peripheral T-cell reactivity to bacterial superantigens in vivo: the response/anergy paradox. Immunol Rev 133:105-117.

94. Fagan, P. K., D. Reinscheid, B. Gottschalk, and G. S. Chhatwal. 2001. Identification and characterization of a novel secreted immunoglobulin binding protein from group A streptococcus. Infect Immun 69:4851-4857. 
95. Stevens, D. L., D. B. Salmi, E. R. McIndoo, and A. E. Bryant. 2000. Molecular epidemiology of nga and NAD glycohydrolase/ADP-ribosyltransferase activity among Streptococcus pyogenes causing streptococcal toxic shock syndrome. $J$ Infect Dis 182:1117-1128.

96. Sitkiewicz, I., M. J. Nagiec, P. Sumby, S. D. Butler, C. Cywes-Bentley, and J. M. Musser. 2006. Emergence of a bacterial clone with enhanced virulence by acquisition of a phage encoding a secreted phospholipase A2. Proc Natl Acad Sci U S A 103:16009-16014.

97. Bohach, G. A., D. J. Fast, R. D. Nelson, and P. M. Schlievert. 1990. Staphylococcal and streptococcal pyrogenic toxins involved in toxic shock syndrome and related illnesses. Crit Rev Microbiol 17:251-272.

98. Marrack, P., and J. Kappler. 1990. The staphylococcal enterotoxins and their relatives. Science 248:705-711.

99. Miethke, T., C. Wahl, K. Heeg, B. Echtenacher, P. H. Krammer, and H. Wagner. 1992. T cell-mediated lethal shock triggered in mice by the superantigen staphylococcal enterotoxin B: critical role of tumor necrosis factor. J Exp Med 175:91-98.

100. Kotb, M. 1995. Bacterial pyrogenic exotoxins as superantigens. Clin Microbiol Rev 8:411-426.

101. Norrby-Teglund, A., P. Thulin, B. S. Gan, M. Kotb, A. McGeer, J. Andersson, and D. E. Low. 2001. Evidence for superantigen involvement in severe group a streptococcal tissue infections. J Infect Dis 184:853-860.

102. Watanabe-Ohnishi, R., D. E. Low, A. McGeer, D. L. Stevens, P. M. Schlievert, D. Newton, B. Schwartz, B. Kreiswirth, and M. Kotb. 1995. Selective depletion of V beta-bearing $\mathrm{T}$ cells in patients with severe invasive group A streptococcal infections and streptococcal toxic shock syndrome. Ontario Streptococcal Study Project. J Infect Dis 171:74-84.

103. Proft, T., and J. D. Fraser. 2003. Bacterial superantigens. Clin Exp Immunol 133:299-306.

104. Thomas, D. Y., S. Jarraud, B. Lemercier, G. Cozon, K. Echasserieau, J. Etienne, M. L. Gougeon, G. Lina, and F. Vandenesch. 2006. Staphylococcal enterotoxinlike toxins $\mathrm{U} 2$ and $\mathrm{V}$, two new staphylococcal superantigens arising from recombination within the enterotoxin gene cluster. Infect Immun 74:4724-4734.

105. Schlievert, P. M., and G. A. Bohach. 2007. Stapylococcal and Streptococcal Superantigens: an Update. ASM Press, Washington, D. C.

106. Proft, T., and J. D. Fraser. 2007. Streptococcal superantigens. Chem Immunol Allergy 93:1-23.

107. Cole, B. C., R. A. Daynes, and J. R. Ward. 1982. Stimulation of mouse lymphocytes by a mitogen derived from Mycoplasma arthritidis. III. Ir gene control of lymphocyte transformation correlates with binding of the mitogen to specific Ia-bearing cells. J Immunol 129:1352-1359.

108. Huang, I. Y., J. L. Hughes, M. S. Bergdoll, and E. J. Schantz. 1987. Complete amino acid sequence of staphylococcal enterotoxin A. J Biol Chem 262:70067013. 
109. Schlievert, P. M., K. N. Shands, B. B. Dan, G. P. Schmid, and R. D. Nishimura. 1981. Identification and characterization of an exotoxin from Staphylococcus aureus associated with toxic-shock syndrome. J Infect Dis 143:509-516.

110. Weeks, C. R., and J. J. Ferretti. 1986. Nucleotide sequence of the type A streptococcal exotoxin (erythrogenic toxin) gene from Streptococcus pyogenes bacteriophage T12. Infect Immun 52:144-150.

111. Bergdoll, M. S. 1956. The chemistry of staphylococcal enterotoxin. Ann N Y Acad Sci 65:139-143.

112. White, J., A. Herman, A. M. Pullen, R. Kubo, J. W. Kappler, and P. Marrack. 1989. The V beta-specific superantigen staphylococcal enterotoxin B: stimulation of mature T cells and clonal deletion in neonatal mice. Cell 56:27-35.

113. Jensen, P. E. 2007. Recent advances in antigen processing and presentation. Nat Immunol 8:1041-1048.

114. Tomai, M. A., P. M. Schlievert, and M. Kotb. 1992. Distinct T-cell receptor V beta gene usage by human T lymphocytes stimulated with the streptococcal pyrogenic exotoxins and pep M5 protein. Infect Immun 60:701-705.

115. Schmidt, K. H., D. Gerlach, L. Wollweber, W. Reichardt, K. Mann, J. H. Ozegowski, and B. Fleischer. 1995. Mitogenicity of M5 protein extracted from Streptococcus pyogenes cells is due to streptococcal pyrogenic exotoxin $\mathrm{C}$ and mitogenic factor MF. Infect Immun 63:4569-4575.

116. Dick, G. F., and G. H. Dick. 1983. Landmark article Jan 26, 1924: The etiology of scarlet fever. By George F. Dick and Gladys Henry Dick. Jama 250:3096.

117. Watson, D. W. 1960. Host-parasite factors in group A streptococcal infections. Pyrogenic and other effects of immunologic distinct exotoxins related to scarlet fever toxins. J Exp Med 111:255-284.

118. Kim, Y. B., and D. W. Watson. 1970. A purified group A streptococcal pyrogenic exotoxin. Physiochemical and biological properties including the enhancement of susceptibility to endotoxin lethal shock. J Exp Med 131:611-622.

119. Abe, J., J. Forrester, T. Nakahara, J. A. Lafferty, B. L. Kotzin, and D. Y. Leung. 1991. Selective stimulation of human T cells with streptococcal erythrogenic toxins A and B. J Immunol 146:3747-3750.

120. Eriksson, A., and M. Norgren. 1999. The superantigenic activity of streptococcal pyrogenic exotoxin B is independent of the protease activity. FEMS Immunol Med Microbiol 25:355-363.

121. Gerlach, D., W. Reichardt, B. Fleischer, and K. H. Schmidt. 1994. Separation of mitogenic and pyrogenic activities from so-called erythrogenic toxin type B (Streptococcal proteinase). Zentralbl Bakteriol 280:507-514.

122. Ferretti, J. J., W. M. McShan, D. Ajdic, D. J. Savic, G. Savic, K. Lyon, C. Primeaux, S. Sezate, A. N. Suvorov, S. Kenton, H. S. Lai, S. P. Lin, Y. Qian, H. G. Jia, F. Z. Najar, Q. Ren, H. Zhu, L. Song, J. White, X. Yuan, S. W. Clifton, B. A. Roe, and R. McLaughlin. 2001. Complete genome sequence of an M1 strain of Streptococcus pyogenes. Proc Natl Acad Sci U S A 98:4658-4663.

123. Proft, T., V. L. Arcus, V. Handley, E. N. Baker, and J. D. Fraser. 2001. Immunological and biochemical characterization of streptococcal pyrogenic exotoxins I and J (SPE-I and SPE-J) from Streptococcus pyogenes. J Immunol 166:6711-6719. 
124. Proft, T., S. L. Moffatt, C. J. Berkahn, and J. D. Fraser. 1999. Identification and characterization of novel superantigens from Streptococcus pyogenes. J Exp Med 189:89-102.

125. Smoot, L. M., J. K. McCormick, J. C. Smoot, N. P. Hoe, I. Strickland, R. L. Cole, K. D. Barbian, C. A. Earhart, D. H. Ohlendorf, L. G. Veasy, H. R. Hill, D. Y. Leung, P. M. Schlievert, and J. M. Musser. 2002. Characterization of two novel pyrogenic toxin superantigens made by an acute rheumatic fever clone of Streptococcus pyogenes associated with multiple disease outbreaks. Infect Immun 70:7095-7104.

126. Beres, S. B., G. L. Sylva, K. D. Barbian, B. Lei, J. S. Hoff, N. D. Mammarella, M. Y. Liu, J. C. Smoot, S. F. Porcella, L. D. Parkins, D. S. Campbell, T. M. Smith, J. K. McCormick, D. Y. Leung, P. M. Schlievert, and J. M. Musser. 2002. Genome sequence of a serotype M3 strain of group A Streptococcus: phage-encoded toxins, the high-virulence phenotype, and clone emergence. Proc Natl Acad Sci U S A 99:10078-10083.

127. Ikebe, T., A. Wada, Y. Inagaki, K. Sugama, R. Suzuki, D. Tanaka, A. Tamaru, Y. Fujinaga, Y. Abe, Y. Shimizu, and H. Watanabe. 2002. Dissemination of the phage-associated novel superantigen gene speL in recent invasive and noninvasive Streptococcus pyogenes M3/T3 isolates in Japan. Infect Immun 70:3227-3233.

128. Proft, T., P. D. Webb, V. Handley, and J. D. Fraser. 2003. Two novel superantigens found in both group A and group C Streptococcus. Infect Immun 71:1361-1369.

129. Hashikawa, S., Y. Iinuma, M. Furushita, T. Ohkura, T. Nada, K. Torii, T. Hasegawa, and M. Ohta. 2004. Characterization of group C and G streptococcal strains that cause streptococcal toxic shock syndrome. J Clin Microbiol 42:186192.

130. Korman, T. M., A. Boers, T. M. Gooding, N. Curtis, and K. Visvanathan. 2004. Fatal case of toxic shock-like syndrome due to group C streptococcus associated with superantigen exotoxin. J Clin Microbiol 42:2866-2869.

131. Ojukwu, I. C., D. W. Newton, A. E. Luque, M. Y. Kotb, and M. Menegus. 2001. Invasive Group C Streptococcus infection associated with rhabdomyolysis and disseminated intravascular coagulation in a previously healthy adult. Scand $J$ Infect Dis 33:227-229.

132. Artiushin, S. C., J. F. Timoney, A. S. Sheoran, and S. K. Muthupalani. 2002. Characterization and immunogenicity of pyrogenic mitogens SePE-H and SePE-I of Streptococcus equi. Microb Pathog 32:71-85.

133. Miyoshi-Akiyama, T., J. Zhao, H. Kato, K. Kikuchi, K. Totsuka, Y. Kataoka, M. Katsumi, and T. Uchiyama. 2003. Streptococcus dysgalactiae-derived mitogen (SDM), a novel bacterial superantigen: characterization of its biological activity and predicted tertiary structure. Mol Microbiol 47:1589-1599.

134. Sachse, S., P. Seidel, D. Gerlach, E. Gunther, J. Rodel, E. Straube, and K. H. Schmidt. 2002. Superantigen-like gene(s) in human pathogenic Streptococcus dysgalactiae, subsp equisimilis: genomic localisation of the gene encoding streptococcal pyrogenic exotoxin $\mathrm{G}$ (speG(dys)). FEMS Immunol Med Microbiol 34:159-167. 
135. Donadini, R., and B. A. Fields. 2007. Yersinia pseudotuberculosis superantigens. Chem Immunol Allergy 93:77-91.

136. Cole, B. C., and C. L. Atkin. 1991. The Mycoplasma arthritidis T-cell mitogen, MAM: a model superantigen. Immunol Today 12:271-276.

137. Ito, Y., J. Abe, K. Yoshino, T. Takeda, and T. Kohsaka. 1995. Sequence analysis of the gene for a novel superantigen produced by Yersinia pseudotuberculosis and expression of the recombinant protein. J Immunol 154:5896-5906.

138. Cole, B. C., and H. Mu. 2007. Mycoplasma Arthritidis-Derived Superantigen (MAM), a Unique Class of Superantigen That Bridges Innate and Adaptive Immunity. ASM Press, Washington, D. C.

139. Li, P. L., R. E. Tiedemann, S. L. Moffat, and J. D. Fraser. 1997. The superantigen streptococcal pyrogenic exotoxin C (SPE-C) exhibits a novel mode of action. $J$ Exp Med 186:375-383.

140. Muller-Alouf, H., T. Proft, T. M. Zollner, D. Gerlach, E. Champagne, P. Desreumaux, C. Fitting, C. Geoffroy-Fauvet, J. E. Alouf, and J. M. Cavaillon. 2001. Pyrogenicity and cytokine-inducing properties of Streptococcus pyogenes superantigens: comparative study of streptococcal mitogenic exotoxin $\mathrm{Z}$ and pyrogenic exotoxin A. Infect Immun 69:4141-4145.

141. Baker, H. M., T. Proft, P. D. Webb, V. L. Arcus, J. D. Fraser, and E. N. Baker. 2004. Crystallographic and mutational data show that the streptococcal pyrogenic exotoxin $\mathrm{J}$ can use a common binding surface for T-cell receptor binding and dimerization. J Biol Chem 279:38571-38576.

142. Eriksson, A., B. Eriksson, S. E. Holm, and M. Norgren. 1999. Streptococcal DNase B is immunologically identical to superantigen SpeF but involves separate domains. Clin Diagn Lab Immunol 6:133-136.

143. Norrby-Teglund, A., D. Newton, M. Kotb, S. E. Holm, and M. Norgren. 1994. Superantigenic properties of the group A streptococcal exotoxin SpeF (MF). Infect Immun 62:5227-5233.

144. Sriskandan, S., M. Unnikrishnan, T. Krausz, and J. Cohen. 2000. Mitogenic factor (MF) is the major DNase of serotype M89 Streptococcus pyogenes. Microbiology 146 (Pt 11):2785-2792.

145. Canchaya, C., C. Proux, G. Fournous, A. Bruttin, and H. Brussow. 2003. Prophage genomics. Microbiol Mol Biol Rev 67:238-276, table of contents.

146. Arcus, V. L., T. Proft, J. A. Sigrell, H. M. Baker, J. D. Fraser, and E. N. Baker. 2000. Conservation and variation in superantigen structure and activity highlighted by the three-dimensional structures of two new superantigens from Streptococcus pyogenes. J Mol Biol 299:157-168.

147. Papageorgiou, A. C., C. M. Collins, D. M. Gutman, J. B. Kline, S. M. O'Brien, H. S. Tranter, and K. R. Acharya. 1999. Structural basis for the recognition of superantigen streptococcal pyrogenic exotoxin A (SpeA1) by MHC class II molecules and T-cell receptors. Embo J 18:9-21.

148. Roussel, A., B. F. Anderson, H. M. Baker, J. D. Fraser, and E. N. Baker. 1997. Crystal structure of the streptococcal superantigen SPE-C: dimerization and zinc binding suggest a novel mode of interaction with MHC class II molecules. Nat Struct Biol 4:635-643. 
149. Sundberg, E., and T. S. Jardetzky. 1999. Structural basis for HLA-DQ binding by the streptococcal superantigen SSA. Nat Struct Biol 6:123-129.

150. Petersson, K., G. Forsberg, and B. Walse. 2004. Interplay between superantigens and immunoreceptors. Scand J Immunol 59:345-355.

151. Li, H., A. Llera, E. L. Malchiodi, and R. A. Mariuzza. 1999. The structural basis of T cell activation by superantigens. Annu Rev Immunol 17:435-466.

152. McCormick, J. K., J. M. Yarwood, and P. M. Schlievert. 2001. Toxic shock syndrome and bacterial superantigens: an update. Annu Rev Microbiol 55:77-104.

153. Brouillard, J. N., S. Gunther, A. K. Varma, I. Gryski, C. A. Herfst, A. K. Rahman, D. Y. Leung, P. M. Schlievert, J. Madrenas, E. J. Sundberg, and J. K. McCormick. 2007. Crystal structure of the streptococcal superantigen SpeI and functional role of a novel loop domain in $\mathrm{T}$ cell activation by group $\mathrm{V}$ superantigens. J Mol Biol 367:925-934.

154. Acharya, K. R., E. F. Passalacqua, E. Y. Jones, K. Harlos, D. I. Stuart, R. D. Brehm, and H. S. Tranter. 1994. Structural basis of superantigen action inferred from crystal structure of toxic-shock syndrome toxin-1. Nature 367:94-97.

155. Kim, J., R. G. Urban, J. L. Strominger, and D. C. Wiley. 1994. Toxic shock syndrome toxin-1 complexed with a class II major histocompatibility molecule HLA-DR1. Science 266:1870-1874.

156. Wen, R., G. A. Cole, S. Surman, M. A. Blackman, and D. L. Woodland. 1996. Major histocompatibility complex class II-associated peptides control the presentation of bacterial superantigens to T cells. J Exp Med 183:1083-1092.

157. Jardetzky, T. S., J. H. Brown, J. C. Gorga, L. J. Stern, R. G. Urban, Y. I. Chi, C. Stauffacher, J. L. Strominger, and D. C. Wiley. 1994. Three-dimensional structure of a human class II histocompatibility molecule complexed with superantigen. Nature 368:711-718.

158. Abrahmsen, L., M. Dohlsten, S. Segren, P. Bjork, E. Jonsson, and T. Kalland. 1995. Characterization of two distinct MHC class II binding sites in the superantigen staphylococcal enterotoxin A. Embo J 14:2978-2986.

159. Petersson, K., M. Hakansson, H. Nilsson, G. Forsberg, L. A. Svensson, A. Liljas, and B. Walse. 2001. Crystal structure of a superantigen bound to MHC class II displays zinc and peptide dependence. Embo J 20:3306-3312.

160. Petersson, K., M. Thunnissen, G. Forsberg, and B. Walse. 2002. Crystal structure of a SEA variant in complex with MHC class II reveals the ability of SEA to crosslink MHC molecules. Structure 10:1619-1626.

161. Gunther, S., A. K. Varma, B. Moza, K. J. Kasper, A. W. Wyatt, P. Zhu, A. K. Rahman, Y. Li, R. A. Mariuzza, J. K. McCormick, and E. J. Sundberg. 2007. A novel loop domain in superantigens extends their $\mathrm{T}$ cell receptor recognition site. J Mol Biol 371:210-221.

162. Li, Y., H. Li, N. Dimasi, J. K. McCormick, R. Martin, P. Schuck, P. M. Schlievert, and R. A. Mariuzza. 2001. Crystal structure of a superantigen bound to the high-affinity, zinc-dependent site on MHC class II. Immunity 14:93-104.

163. Fernandez, M. M., R. Guan, C. P. Swaminathan, E. L. Malchiodi, and R. A. Mariuzza. 2006. Crystal structure of staphylococcal enterotoxin I (SEI) in complex with a human major histocompatibility complex class II molecule. J Biol Chem 281:25356-25364. 
164. Nelson, K., P. M. Schlievert, R. K. Selander, and J. M. Musser. 1991.

Characterization and clonal distribution of four alleles of the speA gene encoding pyrogenic exotoxin A (scarlet fever toxin) in Streptococcus pyogenes. J Exp Med 174:1271-1274.

165. Bessen, D. E., M. W. Izzo, T. R. Fiorentino, R. M. Caringal, S. K. Hollingshead, and B. Beall. 1999. Genetic linkage of exotoxin alleles and emm gene markers for tissue tropism in group A streptococci. J Infect Dis 179:627-636.

166. Proft, T., S. L. Moffatt, K. D. Weller, A. Paterson, D. Martin, and J. D. Fraser. 2000. The streptococcal superantigen SMEZ exhibits wide allelic variation, mosaic structure, and significant antigenic variation. J Exp Med 191:1765-1776.

167. Wen, R., D. R. Broussard, S. Surman, T. L. Hogg, M. A. Blackman, and D. L. Woodland. 1997. Carboxy-terminal residues of major histocompatibility complex class II-associated peptides control the presentation of the bacterial superantigen toxic shock syndrome toxin-1 to T cells. Eur J Immunol 27:772-781.

168. Hogan, R. J., J. VanBeek, D. R. Broussard, S. L. Surman, and D. L. Woodland. 2001. Identification of MHC class II-associated peptides that promote the presentation of toxic shock syndrome toxin-1 to T cells. J Immunol 166:65146522.

169. Andersen, P. S., P. Schuck, E. J. Sundberg, C. Geisler, K. Karjalainen, and R. A. Mariuzza. 2002. Quantifying the energetics of cooperativity in a ternary protein complex. Biochemistry 41:5177-5184.

170. Rahman, A. K., C. A. Herfst, B. Moza, S. R. Shames, L. A. Chau, C. Bueno, J. Madrenas, E. J. Sundberg, and J. K. McCormick. 2006. Molecular basis of TCR selectivity, cross-reactivity, and allelic discrimination by a bacterial superantigen: integrative functional and energetic mapping of the SpeC-Vbeta2.1 molecular interface. J Immunol 177:8595-8603.

171. Al-Daccak, R., K. Mehindate, F. Damdoumi, P. Etongue-Mayer, H. Nilsson, P. Antonsson, M. Sundstrom, M. Dohlsten, R. P. Sekaly, and W. Mourad. 1998. Staphylococcal enterotoxin D is a promiscuous superantigen offering multiple modes of interactions with the MHC class II receptors. J Immunol 160:225-232.

172. Mehindate, K., J. Thibodeau, M. Dohlsten, T. Kalland, R. P. Sekaly, and W. Mourad. 1995. Cross-linking of major histocompatibility complex class II molecules by staphylococcal enterotoxin A superantigen is a requirement for inflammatory cytokine gene expression. J Exp Med 182:1573-1577.

173. Tiedemann, R. E., and J. D. Fraser. 1996. Cross-linking of MHC class II molecules by staphylococcal enterotoxin A is essential for antigen-presenting cell and T cell activation. J Immunol 157:3958-3966.

174. Newton, D. W., M. Dohlsten, C. Olsson, S. Segren, K. E. Lundin, P. A. Lando, T. Kalland, and M. Kotb. 1996. Mutations in the MHC class II binding domains of staphylococcal enterotoxin A differentially affect $\mathrm{T}$ cell receptor Vbeta specificity. J Immunol 157:3988-3994.

175. Sundstrom, M., L. Abrahmsen, P. Antonsson, K. Mehindate, W. Mourad, and M. Dohlsten. 1996. The crystal structure of staphylococcal enterotoxin type D reveals Zn2+-mediated homodimerization. Embo J 15:6832-6840.

176. De Marzi, M. C., M. M. Fernandez, E. J. Sundberg, L. Molinero, N. W. Zwirner, A. S. Llera, R. A. Mariuzza, and E. L. Malchiodi. 2004. Cloning, expression and 
interaction of human T-cell receptors with the bacterial superantigen SSA. Eur J Biochem 271:4075-4083.

177. Baker, M. D., I. Gendlina, C. M. Collins, and K. R. Acharya. 2004. Crystal structure of a dimeric form of streptococcal pyrogenic exotoxin A (SpeA1). Protein Sci 13:2285-2290.

178. Kotb, M., G. Majumdar, M. Tomai, and E. H. Beachey. 1990. Accessory cellindependent stimulation of human $\mathrm{T}$ cells by streptococcal $\mathrm{M}$ protein superantigen. J Immunol 145:1332-1336.

179. Ohnishi, H., T. Tanaka, J. Takahara, and M. Kotb. 1993. CD28 delivers costimulatory signals for superantigen-induced activation of antigen-presenting cell-depleted human T lymphocytes. J Immunol 150:3207-3214.

180. Kotb, M., R. Watanabe-Ohnishi, J. Aelion, T. Tanaka, A. M. Geller, and H. Ohnishi. 1993. Preservation of the specificity of superantigen to $\mathrm{T}$ cell receptor V beta elements in the absence of MHC class II molecules. Cell Immunol 152:348357.

181. Newton, D. W., M. Dohlsten, P. A. Lando, T. Kalland, C. Olsson, and M. Kotb. 1998. MHC class II-independent, Vbeta-specific activation of T cells by superantigen mutants fused to anti-tumor Fab fragments: implications for use in treatment of human colon carcinoma. Int J Mol Med 1:157-162.

182. Antonsson, P., A. G. Wingren, J. Hansson, T. Kalland, M. Varga, and M. Dohlsten. 1997. Functional characterization of the interaction between the superantigen staphylococcal enterotoxin A and the TCR. J Immunol 158:42454251

183. Malchiodi, E. L., E. Eisenstein, B. A. Fields, D. H. Ohlendorf, P. M. Schlievert, K. Karjalainen, and R. A. Mariuzza. 1995. Superantigen binding to a T cell receptor beta chain of known three-dimensional structure. J Exp Med 182:18331845.

184. Woodland, D. L., R. Wen, and M. A. Blackman. 1997. Why do superantigens care about peptides? Immunol Today 18:18-22.

185. Lavoie, P. M., J. Thibodeau, I. Cloutier, R. Busch, and R. P. Sekaly. 1997. Selective binding of bacterial toxins to major histocompatibility complex class IIexpressing cells is controlled by invariant chain and HLA-DM. Proc Natl Acad Sci U S A 94:6892-6897.

186. Kozono, H., D. Parker, J. White, P. Marrack, and J. Kappler. 1995. Multiple binding sites for bacterial superantigens on soluble class II MHC molecules. Immunity 3:187-196.

187. Lavoie, P. M., H. McGrath, N. H. Shoukry, P. A. Cazenave, R. P. Sekaly, and J. Thibodeau. 2001. Quantitative relationship between MHC class II-superantigen complexes and the balance of T cell activation versus death. J Immunol 166:72297237.

188. Kappler, J., B. Kotzin, L. Herron, E. W. Gelfand, R. D. Bigler, A. Boylston, S. Carrel, D. N. Posnett, Y. Choi, and P. Marrack. 1989. V beta-specific stimulation of human T cells by staphylococcal toxins. Science 244:811-813.

189. Tomai, M. A., J. A. Aelion, M. E. Dockter, G. Majumdar, D. G. Spinella, and M. Kotb. 1991. T cell receptor V gene usage by human $T$ cells stimulated with the superantigen streptococcal M protein. J Exp Med 174:285-288. 
190. Leder, L., A. Llera, P. M. Lavoie, M. I. Lebedeva, H. Li, R. P. Sekaly, G. A.

Bohach, P. J. Gahr, P. M. Schlievert, K. Karjalainen, and R. A. Mariuzza. 1998. A mutational analysis of the binding of staphylococcal enterotoxins B and C3 to the $\mathrm{T}$ cell receptor beta chain and major histocompatibility complex class II. $J$ Exp Med 187:823-833.

191. Moza, B., A. K. Varma, R. A. Buonpane, P. Zhu, C. A. Herfst, M. J. Nicholson, A. K. Wilbuer, N. P. Seth, K. W. Wucherpfennig, J. K. McCormick, D. M. Kranz, and E. J. Sundberg. 2007. Structural basis of T-cell specificity and activation by the bacterial superantigen TSST-1. Embo J 26:1187-1197.

192. Li, H., A. Llera, D. Tsuchiya, L. Leder, X. Ysern, P. M. Schlievert, K. Karjalainen, and R. A. Mariuzza. 1998. Three-dimensional structure of the complex between a $\mathrm{T}$ cell receptor beta chain and the superantigen staphylococcal enterotoxin B. Immunity 9:807-816.

193. Petersson, K., H. Pettersson, N. J. Skartved, B. Walse, and G. Forsberg. 2003. Staphylococcal enterotoxin $\mathrm{H}$ induces $\mathrm{V}$ alpha-specific expansion of T cells. $J$ Immunol 170:4148-4154.

194. Pumphrey, N., A. Vuidepot, B. Jakobsen, G. Forsberg, B. Walse, and K. Lindkvist-Petersson. 2007. Cutting edge: Evidence of direct TCR alpha-chain interaction with superantigen. J Immunol 179:2700-2704.

195. Huang, Y., and R. L. Wange. 2004. T cell receptor signaling: beyond complex complexes. J Biol Chem 279:28827-28830.

196. Bueno, C., G. Criado, J. K. McCormick, and J. Madrenas. 2007. T cell signalling induced by bacterial superantigens. Chem Immunol Allergy 93:161-180.

197. Fuller, C. L., and V. L. Braciale. 1998. Selective induction of CD8+ cytotoxic T lymphocyte effector function by staphylococcus enterotoxin B. J Immunol 161:5179-5186.

198. Herrmann, T., and H. R. MacDonald. 1993. The CD8 T cell response to staphylococcal enterotoxins. Semin Immunol 5:33-39.

199. Oyaizu, N., N. Chirmule, H. Yagura, R. Pahwa, R. A. Good, and S. Pahwa. 1992. Superantigen staphylococcal enterotoxin B-induced T-helper cell activation is independent of CD4 molecules and phosphatidylinositol hydrolysis. Proc Natl Acad Sci U S A 89:8035-8039.

200. Bueno, C., C. D. Lemke, G. Criado, M. L. Baroja, S. S. Ferguson, A. K. Rahman, C. D. Tsoukas, J. K. McCormick, and J. Madrenas. 2006. Bacterial superantigens bypass Lck-dependent $\mathrm{T}$ cell receptor signaling by activating a Galpha11dependent, PLC-beta-mediated pathway. Immunity 25:67-78.

201. Criado, G., and J. Madrenas. 2004. Superantigen stimulation reveals the contribution of Lck to negative regulation of T cell activation. J Immunol 172:222-230.

202. Yamasaki, S., M. Tachibana, N. Shinohara, and M. Iwashima. 1997. Lckindependent triggering of T-cell antigen receptor signal transduction by staphylococcal enterotoxins. J Biol Chem 272:14787-14791.

203. al-Ramadi, B. K., T. Nakamura, D. Leitenberg, and A. L. Bothwell. 1996. Deficient expression of p56(lck) in Th2 cells leads to partial TCR signaling and a dysregulation in lymphokine mRNA levels. J Immunol 157:4751-4761. 
204. Yamashita, M., K. Hashimoto, M. Kimura, M. Kubo, T. Tada, and T. Nakayama. 1998. Requirement for p56(lck) tyrosine kinase activation in Th subset differentiation. Int Immunol 10:577-591.

205. Gehring, S., M. Schlaak, and J. van der Bosch. 1998. A new in vitro model for studying human $\mathrm{T}$ cell differentiation: $\mathrm{T}(\mathrm{H} 1) / \mathrm{T}(\mathrm{H} 2)$ induction following activation by superantigens. J Immunol Methods 219:85-98.

206. Norrby-Teglund, A., R. Lustig, and M. Kotb. 1997. Differential induction of Th1 versus Th2 cytokines by group A streptococcal toxic shock syndrome isolates. Infect Immun 65:5209-5215.

207. Majumdar, G., E. H. Beachey, M. Tomai, and M. Kotb. 1990. Differential signal requirements in T-cell activation by mitogen and superantigen. Cell Signal 2:521530 .

208. Tomai, M. A., E. H. Beachey, G. Majumdar, and M. Kotb. 1992. Metabolically active antigen presenting cells are required for human $\mathrm{T}$ cell proliferation in response to the superantigen streptococcal M protein. FEMS Microbiol Immunol 4:155-164.

209. Majumdar, G., H. Ohnishi, M. A. Tomai, A. M. Geller, B. Wang, M. E. Dockter, and M. Kotb. 1993. Role of antigen-presenting cells in activation of human T cells by the streptococcal $\mathrm{M}$ protein superantigen: requirement for secreted and membrane-associated costimulatory factors. Infect Immun 61:785-790.

210. Ohnishi, H., J. A. Ledbetter, S. B. Kanner, P. S. Linsley, T. Tanaka, A. M. Geller, and M. Kotb. 1995. CD28 cross-linking augments TCR-mediated signals and costimulates superantigen responses. J Immunol 154:3180-3193.

211. Darlington, P. J., M. L. Baroja, T. A. Chau, E. Siu, V. Ling, B. M. Carreno, and J. Madrenas. 2002. Surface cytotoxic T lymphocyte-associated antigen 4 partitions within lipid rafts and relocates to the immunological synapse under conditions of inhibition of T cell activation. J Exp Med 195:1337-1347.

212. Krummel, M. F., T. J. Sullivan, and J. P. Allison. 1996. Superantigen responses and co-stimulation: CD28 and CTLA-4 have opposing effects on T cell expansion in vitro and in vivo. Int Immunol 8:519-523.

213. Kotzin, B. L., D. Y. Leung, J. Kappler, and P. Marrack. 1993. Superantigens and their potential role in human disease. Adv Immunol 54:99-166.

214. Schlievert, P. M. 1993. Role of superantigens in human disease. J Infect Dis 167:997-1002.

215. Alber, G., D. K. Hammer, and B. Fleischer. 1990. Relationship between enterotoxic- and $\mathrm{T}$ lymphocyte-stimulating activity of staphylococcal enterotoxin B. J Immunol 144:4501-4506.

216. Schlievert, P. M., L. M. Jablonski, M. Roggiani, I. Sadler, S. Callantine, D. T. Mitchell, D. H. Ohlendorf, and G. A. Bohach. 2000. Pyrogenic toxin superantigen site specificity in toxic shock syndrome and food poisoning in animals. Infect Immun 68:3630-3634.

217. Harris, T. O., D. Grossman, J. W. Kappler, P. Marrack, R. R. Rich, and M. J. Betley. 1993. Lack of complete correlation between emetic and T-cell-stimulatory activities of staphylococcal enterotoxins. Infect Immun 61:3175-3183. 
218. Schlievert, P. M., T. J. Tripp, and M. L. Peterson. 2004. Reemergence of staphylococcal toxic shock syndrome in Minneapolis-St. Paul, Minnesota, during the 2000-2003 surveillance period. J Clin Microbiol 42:2875-2876.

219. Dinges, M. M., P. M. Orwin, and P. M. Schlievert. 2000. Exotoxins of Staphylococcus aureus. Clin Microbiol Rev 13:16-34, table of contents.

220. Bonventre, P. F., H. Heeg, C. Cullen, and C. J. Lian. 1993. Toxicity of recombinant toxic shock syndrome toxin 1 and mutant toxins produced by Staphylococcus aureus in a rabbit infection model of toxic shock syndrome. Infect Immun 61:793-799.

221. Stevens, D. L. 1992. Invasive group A streptococcus infections. Clin Infect Dis 14:2-11.

222. Stevens, D. L. 2000. Streptococcal toxic shock syndrome associated with necrotizing fasciitis. Annu Rev Med 51:271-288.

223. Norrby-Teglund, A., R. Kaul, D. E. Low, A. McGeer, J. Andersson, U. Andersson, and M. Kotb. 1996. Evidence for the presence of streptococcalsuperantigen-neutralizing antibodies in normal polyspecific immunoglobulin G. Infect Immun 64:5395-5398.

224. Kaul, R., A. McGeer, A. Norrby-Teglund, M. Kotb, B. Schwartz, K. O'Rourke, J. Talbot, and D. E. Low. 1999. Intravenous immunoglobulin therapy for streptococcal toxic shock syndrome--a comparative observational study. The Canadian Streptococcal Study Group. Clin Infect Dis 28:800-807.

225. Sriskandan, S., D. Moyes, and J. Cohen. 1996. Detection of circulating bacterial superantigen and lymphotoxin-alpha in patients with streptococcal toxic-shock syndrome. Lancet 348:1315-1316.

226. Unnikrishnan, M., D. M. Altmann, T. Proft, F. Wahid, J. Cohen, J. D. Fraser, and S. Sriskandan. 2002. The bacterial superantigen streptococcal mitogenic exotoxin $\mathrm{Z}$ is the major immunoactive agent of Streptococcus pyogenes. J Immunol 169:2561-2569.

227. Welcher, B. C., J. H. Carra, L. DaSilva, J. Hanson, C. S. David, M. J. Aman, and S. Bavari. 2002. Lethal shock induced by streptococcal pyrogenic exotoxin A in mice transgenic for human leukocyte antigen-DQ8 and human CD4 receptors: implications for development of vaccines and therapeutics. J Infect Dis 186:501510.

228. Lei, L., J. Altstaedt, M. von der Ohe, T. Proft, U. Gross, and L. Rink. 2001. Induction of interleukin-8 in human neutrophils after MHC class II cross-linking with superantigens. J Leukoc Biol 70:80-86.

229. Alouf, J. E., and H. Muller-Alouf. 2003. Staphylococcal and streptococcal superantigens: molecular, biological and clinical aspects. Int J Med Microbiol 292:429-440.

230. Slifka, M. K., and J. L. Whitton. 2000. Clinical implications of dysregulated cytokine production. $J$ Mol Med 78:74-80.

231. Dinges, M. M., and P. M. Schlievert. 2001. Comparative analysis of lipopolysaccharide-induced tumor necrosis factor alpha activity in serum and lethality in mice and rabbits pretreated with the staphylococcal superantigen toxic shock syndrome toxin 1. Infect Immun 69:7169-7172. 
232. Camussi, G., E. Albano, C. Tetta, and F. Bussolino. 1991. The molecular action of tumor necrosis factor-alpha. Eur J Biochem 202:3-14.

233. Dinarello, C. A. 1991. The proinflammatory cytokines interleukin-1 and tumor necrosis factor and treatment of the septic shock syndrome. J Infect Dis 163:1177-1184.

234. Dinarello, C. A., and S. M. Wolff. 1993. The role of interleukin-1 in disease. $N$ Engl J Med 328:106-113.

235. Ting, J. P., and A. S. Baldwin. 1993. Regulation of MHC gene expression. Curr Opin Immunol 5:8-16.

236. Matsubara, K., and T. Fukaya. 2007. The role of superantigens of group A Streptococcus and Staphylococcus aureus in Kawasaki disease. Curr Opin Infect Dis 20:298-303.

237. Meissner, H. C., and D. Y. Leung. 2000. Superantigens, conventional antigens and the etiology of Kawasaki syndrome. Pediatr Infect Dis J 19:91-94.

238. Brogan, P. A., V. Shah, L. A. Clarke, M. J. Dillon, and N. Klein. 2008. T cell activation profiles in Kawasaki syndrome. Clin Exp Immunol 151:267-274.

239. Leung, D. Y., C. Meissner, D. Fulton, and P. M. Schlievert. 1995. The potential role of bacterial superantigens in the pathogenesis of Kawasaki syndrome. J Clin Immunol 15:11S-17S.

240. Matsubara, K., T. Fukaya, K. Miwa, N. Shibayama, H. Nigami, H. Harigaya, H. Nozaki, T. Hirata, K. Baba, T. Suzuki, and A. Ishiguro. 2006. Development of serum IgM antibodies against superantigens of Staphylococcus aureus and Streptococcus pyogenes in Kawasaki disease. Clin Exp Immunol 143:427-434.

241. Stollerman, G. H. 1997. Rheumatic fever. Lancet 349:935-942.

242. Smoot, J. C., K. D. Barbian, J. J. Van Gompel, L. M. Smoot, M. S. Chaussee, G. L. Sylva, D. E. Sturdevant, S. M. Ricklefs, S. F. Porcella, L. D. Parkins, S. B. Beres, D. S. Campbell, T. M. Smith, Q. Zhang, V. Kapur, J. A. Daly, L. G. Veasy, and J. M. Musser. 2002. Genome sequence and comparative microarray analysis of serotype M18 group A Streptococcus strains associated with acute rheumatic fever outbreaks. Proc Natl Acad Sci U S A 99:4668-4673.

243. Hill, A. V. 2006. Aspects of genetic susceptibility to human infectious diseases. Annu Rev Genet 40:469-486.

244. kotb, M. 2004. genetics of susceptibility to infectious diseases. ASM News 70:457-463.

245. Dahmer, M. K., A. Randolph, S. Vitali, and M. W. Quasney. 2005. Genetic polymorphisms in sepsis. Pediatr Crit Care Med 6:S61-73.

246. Frodsham, A. J., and A. V. Hill. 2004. Genetics of infectious diseases. Hum Mol Genet 13 Spec No 2:R187-194.

247. Texereau, J., J. D. Chiche, W. Taylor, G. Choukroun, B. Comba, and J. P. Mira. 2005. The importance of Toll-like receptor 2 polymorphisms in severe infections. Clin Infect Dis 41 Suppl 7:S408-415.

248. Agnese, D. M., J. E. Calvano, S. J. Hahm, S. M. Coyle, S. A. Corbett, S. E. Calvano, and S. F. Lowry. 2002. Human toll-like receptor 4 mutations but not CD14 polymorphisms are associated with an increased risk of gram-negative infections. J Infect Dis 186:1522-1525. 
249. Platonov, A. E., G. A. Shipulin, I. V. Vershinina, J. Dankert, J. G. van de Winkel, and E. J. Kuijper. 1998. Association of human Fe gamma RIIa (CD32)

polymorphism with susceptibility to and severity of meningococcal disease. Clin Infect Dis 27:746-750.

250. Eisen, D. P., and R. M. Minchinton. 2003. Impact of mannose-binding lectin on susceptibility to infectious diseases. Clin Infect Dis 37:1496-1505.

251. Miller, L. H., S. J. Mason, J. A. Dvorak, M. H. McGinniss, and I. K. Rothman. 1975. Erythrocyte receptors for (Plasmodium knowlesi) malaria: Duffy blood group determinants. Science 189:561-563.

252. Miller, L. H., S. J. Mason, D. F. Clyde, and M. H. McGinniss. 1976. The resistance factor to Plasmodium vivax in blacks. The Duffy-blood-group genotype, FyFy. N Engl J Med 295:302-304.

253. Smith, M. W., M. Carrington, C. Winkler, D. Lomb, M. Dean, G. Huttley, and S. J. O'Brien. 1997. CCR2 chemokine receptor and AIDS progression. Nat Med 3:1052-1053.

254. Huang, Y., W. A. Paxton, S. M. Wolinsky, A. U. Neumann, L. Zhang, T. He, S. Kang, D. Ceradini, Z. Jin, K. Yazdanbakhsh, K. Kunstman, D. Erickson, E. Dragon, N. R. Landau, J. Phair, D. D. Ho, and R. A. Koup. 1996. The role of a mutant CCR5 allele in HIV-1 transmission and disease progression. Nat Med 2:1240-1243.

255. An, P., G. W. Nelson, L. Wang, S. Donfield, J. J. Goedert, J. Phair, D. Vlahov, S. Buchbinder, W. L. Farrar, W. Modi, S. J. O'Brien, and C. A. Winkler. 2002. Modulating influence on HIV/AIDS by interacting RANTES gene variants. Proc Natl Acad Sci U S A 99:10002-10007.

256. Winkler, C., W. Modi, M. W. Smith, G. W. Nelson, X. Wu, M. Carrington, M. Dean, T. Honjo, K. Tashiro, D. Yabe, S. Buchbinder, E. Vittinghoff, J. J. Goedert, T. R. O'Brien, L. P. Jacobson, R. Detels, S. Donfield, A. Willoughby, E. Gomperts, D. Vlahov, J. Phair, and S. J. O'Brien. 1998. Genetic restriction of AIDS pathogenesis by an SDF-1 chemokine gene variant. ALIVE Study, Hemophilia Growth and Development Study (HGDS), Multicenter AIDS Cohort Study (MACS), Multicenter Hemophilia Cohort Study (MHCS), San Francisco City Cohort (SFCC). Science 279:389-393.

257. Shin, H. D., C. Winkler, J. C. Stephens, J. Bream, H. Young, J. J. Goedert, T. R. O'Brien, D. Vlahov, S. Buchbinder, J. Giorgi, C. Rinaldo, S. Donfield, A. Willoughby, S. J. O'Brien, and M. W. Smith. 2000. Genetic restriction of HIV-1 pathogenesis to AIDS by promoter alleles of IL10. Proc Natl Acad Sci U S A 97:14467-14472.

258. Saito, T., G. Ji, H. Shinzawa, K. Okumoto, E. Hattori, T. Adachi, T. Takeda, K. Sugahara, J. I. Ito, H. Watanabe, K. Saito, H. Togashi, K. Ishii, T. Matsuura, K. Inageda, M. Muramatsu, and S. Kawata. 2004. Genetic variations in humans associated with differences in the course of hepatitis C. Biochem Biophys Res Commun 317:335-341.

259. Smolnikova, M. V., and V. I. Konenkov. 2002. Association of IL2, TNFA, IL4 and IL10 Promoter Gene Polymorphisms with the Rate of Progression of the HIV Infection. Russ J Immunol 7:349-356. 
260. Nakayama, E. E., Y. Hoshino, X. Xin, H. Liu, M. Goto, N. Watanabe, H. Taguchi, A. Hitani, A. Kawana-Tachikawa, M. Fukushima, K. Yamada, W. Sugiura, S. I. Oka, A. Ajisawa, H. Sato, Y. Takebe, T. Nakamura, Y. Nagai, A. Iwamoto, and T. Shioda. 2000. Polymorphism in the interleukin-4 promoter affects acquisition of human immunodeficiency virus type 1 syncytium-inducing phenotype. J Virol 74:5452-5459.

261. Knapp, S., B. J. Hennig, A. J. Frodsham, L. Zhang, S. Hellier, M. Wright, R. Goldin, A. V. Hill, H. C. Thomas, and M. R. Thursz. 2003. Interleukin-10 promoter polymorphisms and the outcome of hepatitis $\mathrm{C}$ virus infection. Immunogenetics 55:362-369.

262. Lowe, P. R., H. F. Galley, A. Abdel-Fattah, and N. R. Webster. 2003. Influence of interleukin-10 polymorphisms on interleukin-10 expression and survival in critically ill patients. Crit Care Med 31:34-38.

263. Cheong, J. Y., S. W. Cho, I. L. Hwang, S. K. Yoon, J. H. Lee, C. S. Park, J. E. Lee, K. B. Hahm, and J. H. Kim. 2006. Association between chronic hepatitis B virus infection and interleukin-10, tumor necrosis factor-alpha gene promoter polymorphisms. J Gastroenterol Hepatol 21:1163-1169.

264. Roy, S., W. McGuire, C. G. Mascie-Taylor, B. Saha, S. K. Hazra, A. V. Hill, and D. Kwiatkowski. 1997. Tumor necrosis factor promoter polymorphism and susceptibility to lepromatous leprosy. J Infect Dis 176:530-532.

265. Knight, J. C., I. Udalova, A. V. Hill, B. M. Greenwood, N. Peshu, K. Marsh, and D. Kwiatkowski. 1999. A polymorphism that affects OCT-1 binding to the TNF promoter region is associated with severe malaria. Nat Genet 22:145-150.

266. Hohler, T., A. Kruger, G. Gerken, P. M. Schneider, K. H. Meyer zum Buschenfelde, and C. Rittner. 1998. Tumor necrosis factor alpha promoter polymorphism at position -238 is associated with chronic active hepatitis $\mathrm{C}$ infection. J Med Virol 54:173-177.

267. Hohler, T., A. Kruger, G. Gerken, P. M. Schneider, K. H. Meyer zum Buschenefelde, and C. Rittner. 1998. A tumor necrosis factor-alpha (TNF-alpha) promoter polymorphism is associated with chronic hepatitis B infection. Clin Exp Immunol 111:579-582.

268. Altare, F., D. Lammas, P. Revy, E. Jouanguy, R. Doffinger, S. Lamhamedi, P. Drysdale, D. Scheel-Toellner, J. Girdlestone, P. Darbyshire, M. Wadhwa, H. Dockrell, M. Salmon, A. Fischer, A. Durandy, J. L. Casanova, and D. S. Kumararatne. 1998. Inherited interleukin 12 deficiency in a child with bacille Calmette-Guerin and Salmonella enteritidis disseminated infection. J Clin Invest 102:2035-2040.

269. de Jong, R., F. Altare, I. A. Haagen, D. G. Elferink, T. Boer, P. J. van Breda Vriesman, P. J. Kabel, J. M. Draaisma, J. T. van Dissel, F. P. Kroon, J. L. Casanova, and T. H. Ottenhoff. 1998. Severe mycobacterial and Salmonella infections in interleukin-12 receptor-deficient patients. Science 280:1435-1438.

270. Newport, M. J., C. M. Huxley, S. Huston, C. M. Hawrylowicz, B. A. Oostra, R. Williamson, and M. Levin. 1996. A mutation in the interferon-gamma-receptor gene and susceptibility to mycobacterial infection. N Engl J Med 335:1941-1949.

271. Gollob, J. A., K. G. Veenstra, H. Jyonouchi, A. M. Kelly, P. Ferrieri, D. J. Panka, F. Altare, C. Fieschi, J. L. Casanova, D. A. Frank, and J. W. Mier. 2000. 
Impairment of STAT activation by IL-12 in a patient with atypical mycobacterial and staphylococcal infections. J Immunol 165:4120-4126.

272. Dupuis, S., C. Dargemont, C. Fieschi, N. Thomassin, S. Rosenzweig, J. Harris, S. M. Holland, R. D. Schreiber, and J. L. Casanova. 2001. Impairment of mycobacterial but not viral immunity by a germline human STAT1 mutation. Science 293:300-303.

273. Basma, H., A. Norrby-Teglund, Y. Guedez, A. McGeer, D. E. Low, O. ElAhmedy, B. Schwartz, and M. Kotb. 1999. Risk factors in the pathogenesis of invasive group A streptococcal infections: role of protective humoral immunity. Infect Immun 67:1871-1877.

274. Chatellier, S., N. Ihendyane, R. G. Kansal, F. Khambaty, H. Basma, A. NorrbyTeglund, D. E. Low, A. McGeer, and M. Kotb. 2000. Genetic relatedness and superantigen expression in group A streptococcus serotype M1 isolates from patients with severe and nonsevere invasive diseases. Infect Immun 68:35233534.

275. Kotb, M., A. Norrby-Teglund, A. McGeer, H. El-Sherbini, M. T. Dorak, A. Khurshid, K. Green, J. Peeples, J. Wade, G. Thomson, B. Schwartz, and D. E. Low. 2002. An immunogenetic and molecular basis for differences in outcomes of invasive group A streptococcal infections. Nat Med 8:1398-1404.

276. Norrby-Teglund, A., S. Chatellier, D. E. Low, A. McGeer, K. Green, and M. Kotb. 2000. Host variation in cytokine responses to superantigens determine the severity of invasive group A streptococcal infection. Eur J Immunol 30:32473255.

277. Factor, S. H., O. S. Levine, B. Schwartz, L. H. Harrison, M. M. Farley, A. McGeer, and A. Schuchat. 2003. Invasive group A streptococcal disease: risk factors for adults. Emerg Infect Dis 9:970-977.

278. Factor, S. H., O. S. Levine, L. H. Harrison, M. M. Farley, A. McGeer, T. Skoff, C. Wright, B. Schwartz, and A. Schuchat. 2005. Risk factors for pediatric invasive group A streptococcal disease. Emerg Infect Dis 11:1062-1066.

279. Laupland, K. B., H. D. Davies, D. E. Low, B. Schwartz, K. Green, and A. McGeer. 2000. Invasive group A streptococcal disease in children and association with varicella-zoster virus infection. Ontario Group A Streptococcal Study Group. Pediatrics 105:E60.

280. Hill, A. V. 2001. The genomics and genetics of human infectious disease susceptibility. Annu Rev Genomics Hum Genet 2:373-400.

281. Roger, M. 1998. Influence of host genes on HIV-1 disease progression. Faseb J 12:625-632.

282. Cooke, G. S., and A. V. Hill. 2001. Genetics of susceptibility to human infectious disease. Nat Rev Genet 2:967-977.

283. Guedez, Y., A. Kotby, M. El-Demellawy, A. Galal, G. Thomson, S. Zaher, S. Kassem, and M. Kotb. 1999. HLA class II associations with rheumatic heart disease are more evident and consistent among clinically homogeneous patients. Circulation 99:2784-2790.

284. Scholl, P. R., A. Diez, R. Karr, R. P. Sekaly, J. Trowsdale, and R. S. Geha. 1990. Effect of isotypes and allelic polymorphism on the binding of staphylococcal exotoxins to MHC class II molecules. J Immunol 144:226-230. 
285. Cole, B. C., A. D. Sawitzke, E. A. Ahmed, C. L. Atkin, and C. S. David. 1997. Allelic polymorphisms at the H-2A and HLA-DQ loci influence the response of murine lymphocytes to the Mycoplasma arthritidis superantigen MAM. Infect Immun 65:4190-4198.

286. Guo, W., W. Mourad, D. Charron, and R. Al-Daccak. 1999. Ligation of MHC class II molecules differentially upregulates TNF beta gene expression in B cell lines of different MHC class II haplotypes. Hum Immunol 60:312-322.

287. Imanishi, K., H. Igarashi, and T. m. Uchiyama. 1992. Relative abilities of distinct isotypes of human major histocompatibility complex class II molecules to bind streptococcal pyrogenic exotoxin types A and B. Infect Immun 60:5025-5029.

288. Norrby-Teglund, A., G. T. Nepom, and M. Kotb. 2002. Differential presentation of group A streptococcal superantigens by HLA class II DQ and DR alleles. Eur J Immunol 32:2570-2577.

289. Herman, A., G. Croteau, R. P. Sekaly, J. Kappler, and P. Marrack. 1990. HLADR alleles differ in their ability to present staphylococcal enterotoxins to T cells. J Exp Med 172:709-717.

290. Rajagopalan, G., K. Iijima, M. Singh, H. Kita, R. Patel, and C. S. David. 2006. Intranasal exposure to bacterial superantigens induces airway inflammation in HLA class II transgenic mice. Infect Immun 74:1284-1296.

291. Norrby-Teglund, A., M. P. Muller, A. McGeer, B. S. Gan, V. Guru, J. Bohnen, P. Thulin, and D. E. Low. 2005. Successful management of severe group A streptococcal soft tissue infections using an aggressive medical regimen including intravenous polyspecific immunoglobulin together with a conservative surgical approach. Scand J Infect Dis 37:166-172.

292. Low, D. E., Schwartz, B. and Mcgeer, A. 1998. The reemergence of severe group A streptoccal disease: An evolutionary perspective. ASM Press, Washington DC.

293. Kotb, M. 1995. Infection and autoimmunity: a story of the host, the pathogen, and the copathogen. Clin Immunol Immunopathol 74:10-22.

294. Norrby-Teglund, A., R. Kaul, D. E. Low, A. McGeer, D. W. Newton, J. Andersson, U. Andersson, and M. Kotb. 1996. Plasma from patients with severe invasive group A streptococcal infections treated with normal polyspecific IgG inhibits streptococcal superantigen-induced $\mathrm{T}$ cell proliferation and cytokine production. J Immunol 156:3057-3064.

295. Johnson, D. R., J. T. Wotton, A. Shet, and E. L. Kaplan. 2002. A comparison of group A streptococci from invasive and uncomplicated infections: are virulent clones responsible for serious streptococcal infections? J Infect Dis 185:15861595.

296. Blank, C., A. Luz, S. Bendigs, A. Erdmann, H. Wagner, and K. Heeg. 1997. Superantigen and endotoxin synergize in the induction of lethal shock. Eur J Immunol 27:825-833.

297. Sriskandan, S., M. Unnikrishnan, T. Krausz, H. Dewchand, S. Van Noorden, J. Cohen, and D. M. Altmann. 2001. Enhanced susceptibility to superantigenassociated streptococcal sepsis in human leukocyte antigen-DQ transgenic mice. $J$ Infect Dis 184:166-173. 
298. Fremont, D. H., W. A. Hendrickson, P. Marrack, and J. Kappler. 1996. Structures of an MHC class II molecule with covalently bound single peptides. Science 272:1001-1004.

299. Taneja, V., and C. S. David. 1999. HLA class II transgenic mice as models of human diseases. Immunol Rev 169:67-79.

300. DaSilva, L., B. C. Welcher, R. G. Ulrich, M. J. Aman, C. S. David, and S. Bavari. 2002. Humanlike immune response of human leukocyte antigen-DR3 transgenic mice to staphylococcal enterotoxins: a novel model for superantigen vaccines. $J$ Infect Dis 185:1754-1760.

301. Roy, C. J., K. L. Warfield, B. C. Welcher, R. F. Gonzales, T. Larsen, J. Hanson, C. S. David, T. Krakauer, and S. Bavari. 2005. Human leukocyte antigen-DQ8 transgenic mice: a model to examine the toxicity of aerosolized staphylococcal enterotoxin B. Infect Immun 73:2452-2460.

302. Sriskandan, S., M. Ferguson, V. Elliot, L. Faulkner, and J. Cohen. 2006. Human intravenous immunoglobulin for experimental streptococcal toxic shock: bacterial clearance and modulation of inflammation. J Antimicrob Chemother 58:117-124.

303. Bradley, D. S., G. H. Nabozny, S. Cheng, P. Zhou, M. M. Griffiths, H. S. Luthra, and C. S. David. 1997. HLA-DQB1 polymorphism determines incidence, onset, and severity of collagen-induced arthritis in transgenic mice. Implications in human rheumatoid arthritis. J Clin Invest 100:2227-2234.

304. Taneja, V., N. Taneja, M. Behrens, S. Pan, T. Trejo, M. Griffiths, H. Luthra, and C. S. David. 2003. HLA-DRB1*0402 (DW10) transgene protects collageninduced arthritis-susceptible H2Aq and DRB1*0401 (DW4) transgenic mice from arthritis. J Immunol 171:4431-4438.

305. Cheng, S., J. Baisch, C. Krco, S. Savarirayan, J. Hanson, K. Hodgson, M. Smart, and C. David. 1996. Expression and function of HLA-DQ8 (DQA1*0301/DQB1*0302) genes in transgenic mice. Eur J Immunogenet 23:1520 .

306. Szanto, S., T. Bardos, Z. Szabo, C. S. David, E. I. Buzas, K. Mikecz, and T. T. Glant. 2004. Induction of arthritis in HLA-DR4-humanized and HLA-DQ8humanized mice by human cartilage proteoglycan aggrecan but only in the presence of an appropriate (non-MHC) genetic background. Arthritis Rheum 50:1984-1995.

307. Wilson, R. K., E. Lai, P. Concannon, R. K. Barth, and L. E. Hood. 1988. Structure, organization and polymorphism of murine and human T-cell receptor alpha and beta chain gene families. Immunol Rev 101:149-172.

308. Stevens, D. L. 1996. Invasive group A streptococcal disease. Infect Agents Dis 5:157-166.

309. Bryant, A. E., C. R. Bayer, J. D. Huntington, and D. L. Stevens. 2006. Group A streptococcal myonecrosis: increased vimentin expression after skeletal-muscle injury mediates the binding of Streptococcus pyogenes. J Infect Dis 193:16851692.

310. Cleary, P. P., E. L. Kaplan, J. P. Handley, A. Wlazlo, M. H. Kim, A. R. Hauser, and P. M. Schlievert. 1992. Clonal basis for resurgence of serious Streptococcus pyogenes disease in the 1980s. Lancet 339:518-521. 
311. Muotiala, A., H. Seppala, P. Huovinen, and J. Vuopio-Varkila. 1997. Molecular comparison of group A streptococci of T1M1 serotype from invasive and noninvasive infections in Finland. J Infect Dis 175:392-399.

312. Bernal, A., T. Proft, J. D. Fraser, and D. N. Posnett. 1999. Superantigens in human disease. J Clin Immunol 19:149-157.

313. Marrack, P., M. Blackman, E. Kushnir, and J. Kappler. 1990. The toxicity of staphylococcal enterotoxin B in mice is mediated by T cells. J Exp Med 171:455464.

314. Stiles, B. G., S. Bavari, T. Krakauer, and R. G. Ulrich. 1993. Toxicity of staphylococcal enterotoxins potentiated by lipopolysaccharide: major histocompatibility complex class II molecule dependency and cytokine release. Infect Immun 61:5333-5338.

315. Carrington, M., and S. J. O'Brien. 2003. The influence of HLA genotype on AIDS. Annu Rev Med 54:535-551.

316. Shulman, S. T., G. Stollerman, B. Beall, J. B. Dale, and R. R. Tanz. 2006. Temporal changes in streptococcal $\mathrm{M}$ protein types and the near-disappearance of acute rheumatic fever in the United States. Clin Infect Dis 42:441-447.

317. Chhatwal, G. S., and D. J. McMillan. 2005. Uncovering the mysteries of invasive streptococcal diseases. Trends Mol Med 11:152-155.

318. Norrby-Teglund, A., and M. Kotb. 2000. Host-microbe interactions in the pathogenesis of invasive group A streptococcal infections. J Med Microbiol 49:849-852.

319. Nooh, M. M., N. El-Gengehi, R. Kansal, C. S. David, and M. Kotb. 2007. HLA transgenic mice provide evidence for a direct and dominant role of HLA class II variation in modulating the severity of streptococcal sepsis. J Immunol 178:30763083.

320. Irwin, M. J., K. R. Hudson, K. T. Ames, J. D. Fraser, and N. R. Gascoigne. 1993. $\mathrm{T}$-cell receptor beta-chain binding to enterotoxin superantigens. Immunol Rev 131:61-78.

321. Janeway, C. A., Jr. 1991. Selective elements for the $\mathrm{V}$ beta region of the $\mathrm{T}$ cell receptor: Mls and the bacterial toxic mitogens. Adv Immunol 50:1-53.

322. Rowen, L., B. F. Koop, and L. Hood. 1996. The complete 685-kilobase DNA sequence of the human beta T cell receptor locus. Science 272:1755-1762.

323. Nooh, M. M., R. K. Aziz, M. Kotb, A. Eroshkin, W. J. Chuang, T. Proft, and R. Kansal. 2006. Streptococcal mitogenic exotoxin, SmeZ, is the most susceptible M1T1 streptococcal superantigen to degradation by the streptococcal cysteine protease, SpeB. J Biol Chem 281:35281-35288.

324. al-Daccak, R., K. Mehindate, P. E. Poubelle, and W. Mourad. 1994. Signalling via MHC class II molecules selectively induces IL-1 beta over IL-1 receptor antagonist gene expression. Biochem Biophys Res Commun 201:855-860.

325. Mehindate, K., R. al-Daccak, F. Damdoumi, and W. Mourad. 1996. Synergistic effect between CD40 and class II signals overcome the requirement for class II dimerization in superantigen-induced cytokine gene expression. Eur J Immunol 26:2075-2080.

326. Calandra, T., and M. P. Glauser. 1990. Cytokines and septic shock. Diagn Microbiol Infect Dis 13:377-381. 
327. Dinarello, C. A. 1997. Proinflammatory and anti-inflammatory cytokines as mediators in the pathogenesis of septic shock. Chest 112:321S-329S.

328. Litton, M. J., B. Sander, E. Murphy, A. O'Garra, and J. S. Abrams. 1994. Early expression of cytokines in lymph nodes after treatment in vivo with Staphylococcus enterotoxin B. J Immunol Methods 175:47-58.

329. Kotb, M., H. Ohnishi, G. Majumdar, S. Hackett, A. Bryant, G. Higgins, and D. Stevens. 1993. Temporal relationship of cytokine release by peripheral blood mononuclear cells stimulated by the streptococcal superantigen pep M5. Infect Immun 61:1194-1201.

330. Miethke, T., C. Wahl, D. Regele, H. Gaus, K. Heeg, and H. Wagner. 1993. Superantigen mediated shock: a cytokine release syndrome. Immunobiology 189:270-284.

331. Mourad, W., R. al-Daccak, T. Chatila, and R. S. Geha. 1993. Staphylococcal superantigens as inducers of signal transduction in MHC class II-positive cells. Semin Immunol 5:47-55.

332. Al-Daccak, R., N. Mooney, and D. Charron. 2004. MHC class II signaling in antigen-presenting cells. Curr Opin Immunol 16:108-113.

333. Leveille, C., J. G. Castaigne, D. Charron, and R. Al-Daccak. 2002. MHC class II isotype-specific signaling complex on human B cells. Eur J Immunol 32:22822291.

334. Scumpia, P. O., and L. L. Moldawer. 2005. Biology of interleukin-10 and its regulatory roles in sepsis syndromes. Crit Care Med 33:S468-471.

335. Moore, K. W., R. de Waal Malefyt, R. L. Coffman, and A. O'Garra. 2001. Interleukin-10 and the interleukin-10 receptor. Annu Rev Immunol 19:683-765.

336. de Waal Malefyt, R., J. Haanen, H. Spits, M. G. Roncarolo, A. te Velde, C. Figdor, K. Johnson, R. Kastelein, H. Yssel, and J. E. de Vries. 1991. Interleukin 10 (IL-10) and viral IL-10 strongly reduce antigen-specific human T cell proliferation by diminishing the antigen-presenting capacity of monocytes via downregulation of class II major histocompatibility complex expression. J Exp Med 174:915-924.

337. Bjerre, A., B. Brusletto, E. A. Hoiby, P. Kierulf, and P. Brandtzaeg. 2004. Plasma interferon-gamma and interleukin-10 concentrations in systemic meningococcal disease compared with severe systemic Gram-positive septic shock. Crit Care Med 32:433-438.

338. Gerard, C., C. Bruyns, A. Marchant, D. Abramowicz, P. Vandenabeele, A. Delvaux, W. Fiers, M. Goldman, and T. Velu. 1993. Interleukin 10 reduces the release of tumor necrosis factor and prevents lethality in experimental endotoxemia. $J$ Exp Med 177:547-550.

339. Howard, M., T. Muchamuel, S. Andrade, and S. Menon. 1993. Interleukin 10 protects mice from lethal endotoxemia. $J$ Exp Med 177:1205-1208.

340. Elenkov, I. J., D. G. Iezzoni, A. Daly, A. G. Harris, and G. P. Chrousos. 2005. Cytokine dysregulation, inflammation and well-being. Neuroimmunomodulation 12:255-269.

341. Steinhauser, M. L., C. M. Hogaboam, S. L. Kunkel, N. W. Lukacs, R. M. Strieter, and T. J. Standiford. 1999. IL-10 is a major mediator of sepsis-induced impairment in lung antibacterial host defense. J Immunol 162:392-399. 
342. van der Poll, T., A. Marchant, C. V. Keogh, M. Goldman, and S. F. Lowry. 1996. Interleukin-10 impairs host defense in murine pneumococcal pneumonia. J Infect Dis 174:994-1000.

343. Ashare, A., L. S. Powers, N. S. Butler, K. C. Doerschug, M. M. Monick, and G. W. Hunninghake. 2005. Anti-inflammatory response is associated with mortality and severity of infection in sepsis. Am J Physiol Lung Cell Mol Physiol 288:L633640.

344. Walley, K. R., N. W. Lukacs, T. J. Standiford, R. M. Strieter, and S. L. Kunkel. 1996. Balance of inflammatory cytokines related to severity and mortality of murine sepsis. Infect Immun 64:4733-4738.

345. Lazarus, M., A. H. Hajeer, D. Turner, P. Sinnott, J. Worthington, W. E. Ollier, and I. V. Hutchinson. 1997. Genetic variation in the interleukin 10 gene promoter and systemic lupus erythematosus. J Rheumatol 24:2314-2317.

346. Stanilova, S. A., L. D. Miteva, Z. T. Karakolev, and C. S. Stefanov. 2006. Interleukin-10-1082 promoter polymorphism in association with cytokine production and sepsis susceptibility. Intensive Care Med 32:260-266.

347. Turner, D. M., D. M. Williams, D. Sankaran, M. Lazarus, P. J. Sinnott, and I. V. Hutchinson. 1997. An investigation of polymorphism in the interleukin-10 gene promoter. Eur J Immunogenet 24:1-8.

348. Aziz, R. K., R. Kansal, N. F. Abdeltawab, S. L. Rowe, Y. Su, D. Carrigan, M. M. Nooh, R. R. Attia, C. Brannen, L. A. Gardner, L. Lu, R. W. Williams, and M. Kotb. 2007. Susceptibility to severe streptococcal sepsis: use of a large set of isogenic mouse lines to study genetic and environmental factors. Genes Immun.

349. Proft, T., S. Sriskandan, L. Yang, and J. D. Fraser. 2003. Superantigens and streptococcal toxic shock syndrome. Emerg Infect Dis 9:1211-1218.

350. Donnelly, M. L., G. Luke, A. Mehrotra, X. Li, L. E. Hughes, D. Gani, and M. D. Ryan. 2001. Analysis of the aphthovirus 2A/2B polyprotein 'cleavage' mechanism indicates not a proteolytic reaction, but a novel translational effect: a putative ribosomal 'skip'. J Gen Virol 82:1013-1025.

351. De Felipe, P., and M. Izquierdo. 2000. Tricistronic and tetracistronic retroviral vectors for gene transfer. Hum Gene Ther 11:1921-1931.

352. Szymczak, A. L., C. J. Workman, Y. Wang, K. M. Vignali, S. Dilioglou, E. F. Vanin, and D. A. Vignali. 2004. Correction of multi-gene deficiency in vivo using a single 'self-cleaving' 2A peptide-based retroviral vector. Nat Biotechnol 22:589594.

353. Kaul, R., A. McGeer, D. E. Low, K. Green, and B. Schwartz. 1997. Populationbased surveillance for group A streptococcal necrotizing fasciitis: Clinical features, prognostic indicators, and microbiologic analysis of seventy-seven cases. Ontario Group A Streptococcal Study. Am J Med 103:18-24.

354. Kotb, M. 1998. Superantigens of gram-positive bacteria: structure-function analyses and their implications for biological activity. Curr Opin Microbiol 1:5665.

355. Feske, S., H. Okamura, P. G. Hogan, and A. Rao. 2003. Ca2+/calcineurin signalling in cells of the immune system. Biochem Biophys Res Commun 311:1117-1132. 
356. Gallo, E. M., K. Cante-Barrett, and G. R. Crabtree. 2006. Lymphocyte calcium signaling from membrane to nucleus. Nat Immunol 7:25-32.

357. Lewis, R. S. 2001. Calcium signaling mechanisms in T lymphocytes. Annu Rev Immunol 19:497-521.

358. Randriamampita, C., and A. Trautmann. 2004. Ca2+ signals and T lymphocytes; "New mechanisms and functions in Ca2+ signalling". Biol Cell 96:69-78.

359. Yang, L., M. Thomas, A. Woodhouse, D. Martin, J. D. Fraser, and T. Proft. 2005. Involvement of streptococcal mitogenic exotoxin $\mathrm{Z}$ in streptococcal toxic shock syndrome. J Clin Microbiol 43:3570-3573.

360. Berge, A., and L. Bjorck. 1995. Streptococcal cysteine proteinase releases biologically active fragments of streptococcal surface proteins. J Biol Chem 270:9862-9867.

361. Raeder, R., M. Woischnik, A. Podbielski, and M. D. Boyle. 1998. A secreted streptococcal cysteine protease can cleave a surface-expressed M1 protein and alter the immunoglobulin binding properties. Res Microbiol 149:539-548.

362. Aziz, R. K., M. J. Pabst, A. Jeng, R. Kansal, D. E. Low, V. Nizet, and M. Kotb. 2004. Invasive M1T1 group A Streptococcus undergoes a phase-shift in vivo to prevent proteolytic degradation of multiple virulence factors by SpeB. Mol Microbiol 51:123-134.

363. Kansal, R. G., V. Nizet, A. Jeng, W. J. Chuang, and M. a. Kotb. 2003. Selective modulation of superantigen-induced responses by streptococcal cysteine protease. J Infect Dis 187:398-407.

364. Cherchi, G. B., E. L. Kaplan, P. M. Schlievert, A. Bitti, and G. Orefici. 1992. First reported case of Streptococcus pyogenes infection with toxic shock-like syndrome in Italy. Eur J Clin Microbiol Infect Dis 11:836-838.

365. Linding, R., R. B. Russell, V. Neduva, and T. J. Gibson. 2003. GlobPlot: Exploring protein sequences for globularity and disorder. Nucleic Acids Res 31:3701-3708.

366. Ward, J. J., L. J. McGuffin, K. Bryson, B. F. Buxton, and D. T. Jones. 2004. The DISOPRED server for the prediction of protein disorder. Bioinformatics 20:21382139.

367. Schwede, T., J. Kopp, N. Guex, and M. C. Peitsch. 2003. SWISS-MODEL: An automated protein homology-modeling server. Nucleic Acids Res 31:3381-3385.

368. Chen, C. Y., S. C. Luo, C. F. Kuo, Y. S. Lin, J. J. Wu, M. T. Lin, C. C. Liu, W. Y. Jeng, and W. J. Chuang. 2003. Maturation processing and characterization of streptopain. J Biol Chem 278:17336-17343.

369. Nomizu, M., G. Pietrzynski, T. Kato, P. Lachance, R. Menard, and E. Ziomek. 2001. Substrate specificity of the streptococcal cysteine protease. J Biol Chem 276:44551-44556.

370. Hubbard, S. J., F. Eisenmenger, and J. M. Thornton. 1994. Modeling studies of the change in conformation required for cleavage of limited proteolytic sites. Protein Sci 3:757-768.

371. Kreikemeyer, B., K. S. McIver, and A. Podbielski. 2003. Virulence factor regulation and regulatory networks in Streptococcus pyogenes and their impact on pathogen-host interactions. Trends Microbiol 11:224-232. 
372. Rosch, J. W., and M. G. Caparon. 2005. The ExPortal: an organelle dedicated to the biogenesis of secreted proteins in Streptococcus pyogenes. Mol Microbiol 58:959-968.

373. Rao, M. B., A. M. Tanksale, M. S. Ghatge, and V. V. Deshpande. 1998. Molecular and biotechnological aspects of microbial proteases. Microbiol Mol Biol Rev 62:597-635.

374. Kagawa, T. F., J. C. Cooney, H. M. Baker, S. McSweeney, M. Liu, S. Gubba, J. M. Musser, and E. N. Baker. 2000. Crystal structure of the zymogen form of the group A Streptococcus virulence factor SpeB: an integrin-binding cysteine protease. Proc Natl Acad Sci U S A 97:2235-2240.

375. Collin, M., and A. Olsen. 2003. Extracellular enzymes with immunomodulating activities: variations on a theme in Streptococcus pyogenes. Infect Immun 71:2983-2992.

376. Rasmussen, M., and L. Bjorck. 2002. Proteolysis and its regulation at the surface of Streptococcus pyogenes. Mol Microbiol 43:537-544.

377. Sumby, P., A. R. Whitney, E. A. Graviss, F. R. DeLeo, and J. M. Musser. 2006. Genome-wide analysis of group a streptococci reveals a mutation that modulates global phenotype and disease specificity. PLoS Pathog 2:e5.

378. Green, N. M., S. Zhang, S. F. Porcella, M. J. Nagiec, K. D. Barbian, S. B. Beres, R. B. LeFebvre, and J. M. Musser. 2005. Genome sequence of a serotype M28 strain of group a streptococcus: potential new insights into puerperal sepsis and bacterial disease specificity. J Infect Dis 192:760-770.

379. Yu, C. E., and J. J. Ferretti. 1989. Molecular epidemiologic analysis of the type A streptococcal exotoxin (erythrogenic toxin) gene (speA) in clinical Streptococcus pyogenes strains. Infect Immun 57:3715-3719.

380. Kim, S. W., J. E. Grant, S. I. Kim, T. A. Swanson, G. A. Bernstein, W. B. Jaszcz, K. A. Williams, and P. M. Schlievert. 2004. A possible association of recurrent streptococcal infections and acute onset of obsessive-compulsive disorder. $J$ Neuropsychiatry Clin Neurosci 16:252-260.

381. Rajagopalan, G., G. Polich, M. M. Sen, M. Singh, B. E. Epstein, A. K. Lytle, M. S. Rouse, R. Patel, and C. S. David. 2008. Evaluating the role of HLA-DQ polymorphisms on immune response to bacterial superantigens using transgenic mice. Tissue Antigens 71:135-145.

382. Proft, T., B. Schrage, and J. D. Fraser. 2007. The cytokine response to streptococcal superantigens varies between individual toxins and between individuals: implications for the pathogenesis of group A streptococcal diseases. $J$ Interferon Cytokine Res 27:553-557.

383. Kansal, R. G., A. McGeer, D. E. Low, A. Norrby-Teglund, and M. Kotb. 2000. Inverse relation between disease severity and expression of the streptococcal cysteine protease, SpeB, among clonal M1T1 isolates recovered from invasive group A streptococcal infection cases. Infect Immun 68:6362-6369. 


\section{VITA}

Mohammed Mostafa Nooh was born in Cairo, Egypt, on May 2. 1974. He graduated from the Alibrahemiah High School, Garden City, Cairo, in 1992 and received his Bachelor's degree in Pharmaceutical Sciences from Cairo University in 1997. He enrolled in the Department of Molecular Sciences at the University of Tennessee Health Science Center, in Memphis, in August 2002. 